\title{
Quantifizierung der Feinwurzeln von Bäumen durch elektrische Verfahren
}

Dissertation zur Erlangung des Doktorgrades der Fakultät für Forstwissenschaften und Waldökologie Georg-August-Universität Göttingen

vorgelegt von

Andreas Koch

geboren in Geseke

Göttingen, im Februar 2015 
1. Gutachter: Prof. Dr. Christian Ammer

2. Gutachter: Prof. Dr. Steffen Rust

Tag der mündlichen Prüfung: 15.04.2015 


\section{Danksagung}

Einige Menschen, ohne die die vorliegende Arbeit nicht zustande gekommen wäre, sollen hier dankend erwähnt werden.

Herrn Prof. Dr. Christian Ammer, Erstgutachter meiner Arbeit, möchte ich für die unkomplizierte Unterstützung und Betreuung während der gesamten Bearbeitungszeit danken.

Meinem Zweitgutachter, Herrn Prof. Dr. Steffen Rust von der HAWK, danke ich für die Gelegenheit im Projekt „Städtischer Wurzelraum“ diese Dissertation zu erarbeiten. Ihm gilt besonderer Dank für die wertvolle fachliche Unterstützung sowie die Geduld mit meinen Fragen und Zweifeln.

Herrn Prof. Dr. Dirk Hölscher danke ich für die Übernahme der Rolle des Drittprüfers.

Herrn Dr. Falko Kuhnke danke ich in besonderer Weise. Mit zahlreichen wertvollen Programmen für Messungen und Auswertungen von Daten unterstützte er meine Arbeit. Ohne seine Hilfe wäre die technische Umsetzung der Messysteme in dieser Weise nicht möglich gewesen. Als Ansprechpartner für physikalische Fragen war er unverzichtbar. Weiterhin danke ich ihm für die kritische Durchsicht meiner Ausarbeitungen und die wertvollen Anregungen.

Tapani Repo, Ph.D., vom METLA in Joensuu (Finnland) danke ich für den offenen und konstruktiven Austausch über unsere Forschungsarbeiten, die Gastfreundschaft und die Bereitstellung einiger Exemplare von Salix schwerinii.

Bei Herrn Prof. Dr. Stefan Schütz (Abteilung Forstzoologie und Waldschutz) bedanke ich mich für die Zurverfügungstellung der Klimakammer. Herrn Dr. Bernhard Weißbecker und Herrn Reinhold Dankworth danke ich für die stets spontane und praktische Hilfe bei den technischen Problemen.

Andreas Parth (Abteilung Waldbau und Waldökologie der gemäßigten Zonen) danke ich für die Hilfestellungen bei der Fotoauswertung.

Herr Joachim Koppen von der Fa. Biolab in Braunschweig unterstützte mich freundlicherweise bei der Analyse der Stecklingsnadeln im REM.

Meine Arbeit wurde im Rahmen des angewandten Forschungsschwerpunkts „,Städtischer Wurzelraum“ durch Mittel des „Niedersächsischen Vorab“ der Volkswagenstiftung ermöglicht. Für deren Bereitstellung bedanke ich mich bei der Arbeitsgemeinschaft für innovative Projekte (AGiP) in Hannover.

Mit Frau Dr. Katharina Weltecke und Herrn Dr. Mitja Vianden verbindet mich die schöne gemeinsame Zeit an der HAWK in der „Doktorandenbaracke“. Bei ihnen bin ich stets auf offene Ohren für meine Probleme gestoßen. Corinna Ebeling hat mir bei einigen „R-Problemen“ geholfen. Herzlichen Dank dafür.

Dem „Städtischen Wurzelraum“, den Mitarbeiterinnen der Bibliothek sowie zahlreichen weiteren Angestellten an der HAWK danke ich für aufmunternde Worte und schnelle, unkomplizierte Hilfe. 


\section{Danksagung}

Als studentische Hilfskräfte haben mich Fabian Küdde und Martin Reuter bei zahlreichen Messungen vorbildlich unterstützt.

Meiner Familie und meiner Lebensgefährtin Silke Neumeyer danke ich für die mentale Unterstüzung besonders in den letzten Monaten des Promotionsvorhabens. 


\section{Inhaltsverzeichnis}

Danksagung

Abbildungsverzeichnis v v v v v v v

Tabellenverzeichnis vii

Abkürzungsverzeichnis $\quad$ ix

\begin{tabular}{lll}
\hline 1 & Einleitung & 1
\end{tabular}

2 Stand des Wissens 5

2.1 Aufbau und Funktion einer Pflanzenwurzel . . . . . . . . . . . . . . . . 5

$2.1 .1 \quad$ Morphologie und Anatomie . . . . . . . . . . . . . . . 5

$2.1 .2 \quad$ Wurzelwachstum . . . . . . . . . . . . . . . . . . 7

$2.1 .3 \quad$ Wasseraufnahme und -transport in der Pflanze . . . . . . . . . . . 8

2.2 Übergangswiderstände $\ldots \ldots \ldots \ldots \ldots \ldots$

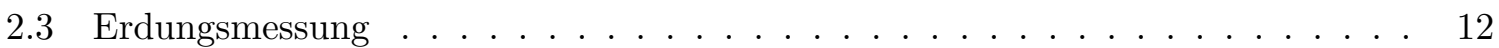

2.4 Bodenwiderstandsmethode . . . . . . . . . . . . . . . . . . . . . . . . . . 14

2.5 Impedanzspektroskopie und kapazitive Messverfahren . . . . . . . . . . . . . 15

3 Material und Methoden 27

3.1 Erdungsmessung . . . . . . . . . . . . . . . . . . . . . . . . . . . . . . 27

3.2 Bodenwiderstandsmethode . . . . . . . . . . . . . . . . . . . . 30

3.2 .1 Modellversuche im elektrolytischen Trog . . . . . . . . . . . . . . . . . . 31

3.2 .2 Feldversuch . . . . . . . . . . . . . . . . . . . . . . 33

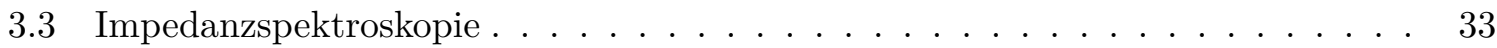

$3.3 .1 \quad$ Messsystem . . . . . . . . . . . . . . . . . . . . . . . . 34

3.3 .2 Darstellung der Messwerte . . . . . . . . . . . . . . . . . . 37

$3.3 .3 \quad$ Differenzbildung von Impedanzspektren $\ldots \ldots \ldots$. . . . . . . . . . . . . 39

3.3 .4 Vorversuche zur Untersuchungen der Randbedingungen . . . . . . . . . 40

3.3 .5 Hauptversuche . . . . . . . . . . . . . . . . . . . 45

3.4 Verwendete Programme $\ldots \ldots \ldots \ldots$. . . . . . . . . . . . . . 49

3.5 Statistische Analysen . . . . . . . . . . . . . . . . . . . . . 51

4 Ergebnisse 53

4.1 Erdungsmessung $\ldots \ldots \ldots \ldots \ldots \ldots$

$4.1 .1 \quad$ Einfluss der Elektrodenkonfiguration $\ldots \ldots \ldots \ldots \ldots$ 
$4.1 .2 \quad$ Einfluss anderer Messgrößen $\ldots \ldots \ldots \ldots \ldots$

4.1 .3 Hauptversuch . . . . . . . . . . . . . . . . . . . . 56

4.2 Bodenwiderstandmethode . . . . . . . . . . . . . . . . . . . . . . . 57

$4.2 .1 \quad$ Modellversuche im elektrolytischen Trog . . . . . . . . . . . . . . . . 57

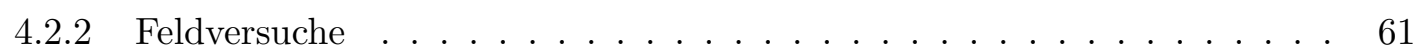

4.3 Impedanzspektroskopie . . . . . . . . . . . . . . . . . . . . . . . . 62

4.3 .1 Überprüfung des Messsystems . . . . . . . . . . . . . . . . . . . . . . 62

4.3 .2 Vorversuche zur Untersuchung der Randbedingungen . . . . . . . . . . . 64

$4.3 .3 \quad$ Hauptversuche . . . . . . . . . . . . . . . . . . . . . . . . . . . . 79

$\begin{array}{lll}5 & \text { Diskussion } & 107\end{array}$

5.1 Erdungsmessung . . . . . . . . . . . . . . . . . . . . . . 107

5.2 Bodenwiderstandsmethode . . . . . . . . . . . . . . . . . . . . . . . . . . 109

5.3 Impedanzspektroskopie . . . . . . . . . . . . . . . . . . . . . . . . . . 111

$5.3 .1 \quad$ Vorversuche zur Untersuchung der Randbedingungen. . . . . . . . . . . . 112

5.3 .2 Hauptversuche . . . . . . . . . . . . . . . . . . . . 117

$\begin{array}{lll}6 & \text { Fazit und Ausblick } & 127\end{array}$

\begin{tabular}{llr}
\hline 7 & Zusammenfassung & 129
\end{tabular}

Literatur $\quad$ xi

A Anhang $\quad$ xvii 


\section{Abbildungsverzeichnis}

2.1 Schematische Darstellungen einer Wurzelspitze. . . . . . . . . . . . . . 7

$2.2 \quad$ Schematische Abbildung des Wassertransports in einer höheren Pflanze. . . . . 9

2.3 Ersatzschaltbild für Zwei- und Vierpunktmessungen. . . . . . . . . . . . . . . 11

2.4 Verschiedenen Darstellung von Spannung und Strom. . . . . . . . . . . . . . . . 16

2.5 Darstellung von $\mathrm{Z}$ und $\phi$ als Zeigerdiagramm. . . . . . . . . . . . . . . . . . . 18

2.6 Veranschaulichung des Vorgehens bei der Modellanpassung. . . . . . . . . . . . 21

$2.7 \quad$ Ersatzschaltbild des double shell models. . . . . . . . . . . . . . . . . . . . 22

2.8 Ersatzschaltbilder für unbewurzelte und bewurzelte Stecklinge (Cao u. a. (2011)). 23

2.9 Ersatzschaltbild aus Ozier-Lafontaine und Bajazet (2005). . . . . . . . . . 24

3.1 Schema der Erdungsmessung am Baum. . . . . . . . . . . . . . . . . . . . . . . . . . . . 28

3.2 Schema der Bodenwiderstandsmethode . . . . . . . . . . . . . . . . . . 31

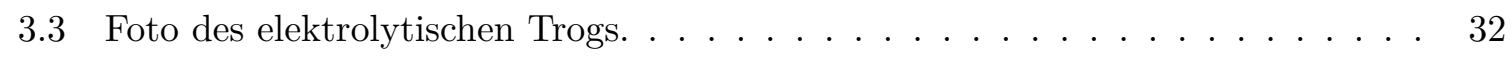

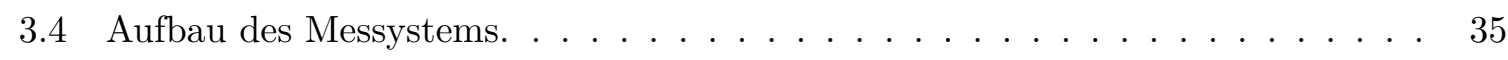

3.5 Ersatzschaltbild der Testschaltung. . . . . . . . . . . . . . . . . . 37

$3.6 \quad$ Verschiedene Darstellungsweisen einer Messung an einer Testschaltung. . . . . . 38

3.7 3-D Darstellung der R,RC-Testschaltung. $\ldots \ldots \ldots \ldots$. . . . . . . . . . . . . . . . . . . . . . . . .

3.8 Grundversuchsaufbau für die Vorversuche. . . . . . . . . . . . . . . . . . . . . . 41

3.9 Fotos des Hauptversuchs 1 in der Klimakammer. . . . . . . . . . . . . . . . 46

3.10 Schema der Mariotteschen Flasche im Versuchsaufbau. . . . . . . . . . . . . . . 48

$4.1 \quad$ Erdungswiderstände von 5 Sommerlinden. . . . . . . . . . . . . . . . . . . . . . . . . . . 54

$4.2 \quad$ Erdungsmessungen an einem Mammutbaum. . . . . . . . . . . . . . . 55

4.3 Zusammenhang zwischen Erdungswiderstand und anderen Messgrößen. . . . . . 56

$4.4 \quad$ Zusammenhang zwischen BHD und Erdungswiderstand. . . . . . . . . . . . . . 57

$4.5 \quad$ Ergebnisse der Messung und der Modellierung an Kupferkabels. . . . . . . . . . 58

$4.6 \quad$ Ergebnisse des Modellversuchs an zwei Kupferscheiben. . . . . . . . . . . . . 59

$4.7 \quad$ Vergleich von Daten zweier Kupferscheiben in verschiedenen Tiefen. . . . . . . 60

4.8 Vergleich von Daten zweier Kupferscheiben in verschiedenen Tiefen. . . . . . . 61

4.9 Bodenwiderstandsmessung an einem Amberbaum. . . . . . . . . . . . . . . 61

4.10 Vergleich zwischen gemessenen und errechneten Spektren. . . . . . . . . . . . . . 63

4.11 Visueller Vergleich dreier Nadeln. . . . . . . . . . . . . . . . . . . . . . 64

4.12 Analyse der Elektrodenoberfläche mit dem Rasterelektronenmikroskop. . . . . . 65

4.13 Vergleich von Messungen in verschiedenen Elektrodenkonfigurationen. . . . . . 66

4.14 Vergleich impedanzspektroskopischer Untersuchungen an einem Steckling. . . . 67

4.15 Ergebnisse des Nährlösungsversuches. … . . . . . . . . . . . . . . . . 69 
4.16 Veränderung des Realteils bei Änderung der Eintauchtiefe. . . . . . . . . . . . . 70

4.17 Darstellung des Imaginärteils über dem Realteil von verschiedenen Stecklingen. 71

4.18 Verlauf von Realteil und Temperatur bei sechs versch. Frequenzen. . . . . . . . 73

4.19 Vergleich von Real- und Imaginärteil der Impedanz bei Tag und Nacht. . . . . 75

4.20 Zusammenhang zwischen Durchmesser und Impedanz. . . . . . . . . . . . . 77

4.21 Vergleich von Real- und Imaginärteil bei versch. Messhöhen an zwei Stecklingen. 78

4.22 Verlauf des Wurzelwachstums eines ausgewählten Stecklings. . . . . . . . . . 79

4.23 Auswertung der Daten aus WinRHIZO ${ }^{\circledR}$ für Hauptversuch 1. . . . . . . . . . . . 81

4.24 Zusammenhang zwischen Wurzellänge und Wurzeloberfläche. . . . . . . . . . . 82

4.25 Auswertung der Daten aus WinRHIZO ${ }^{\circledR}$ für Hauptversuch $2 . \quad$. . . . . . . . . . 83

4.26 Verlauf von Real- und Imaginärteil bei versch. Frequenzen (Hauptversuch 1). . 85

4.27 Ausgewählte Messergebnisse aus Hauptversuch 1 in der komplexen Ebene. . . . 86

4.28 Zusammenhang zw. Real- resp. Imaginärteil u. Wurzellänge (Hauptversuch 1). 87

4.29 Verlauf von Real- und Imaginärteil bei versch. Frequenzen (Hauptversuch 2). . 91

4.30 Zusammenhang zw. Real- resp. Imaginärteil u. Wurzellänge (Hauptversuch 2). 93

4.31 Ausgewählte Messergebnisse aus Hauptversuch 2 in der komplexen Ebene. . . . 95

4.32 Darstellung von Modelldaten u. a. für Hauptversuch $1 . \ldots$. . . . . . . . . . . 96

4.33 Multivariate Analyse der Modelldaten für Hauptversuch 1. . . . . . . . . . . . . 99

4.34 Darstellung von Modelldaten u. a. für Hauptversuch 2. . . . . . . . . . . . . . . 101

4.35 Multivariate Analyse der Zweipunkt-Modelldaten für Hauptversuch 2. . . . . . 104

4.36 Multivariate Analyse der Dreipunkt-Modelldaten für Hauptversuch 2. . . . . . 105

5.1 Vorwärtsrechnung bei Variation verschiedener Parameter. . . . . . . . . . . . 116

$5.2 \quad$ Vergleich von Vorwärtsdaten mit Messdaten aus Hauptversuch 2. . . . . . . . . 120

A.1 Bilder der Edelstahlkanülenoberfläche (REM). . . . . . . . . . . . . . . . . xvii

A.2 Stecklinge 3 bis 6 aus dem Nährlösungsversuch. . . . . . . . . . . . . . . . . xviii

A.3 Stecklinge 7 bis 10 aus dem Nährlösungsversuch. . . . . . . . . . . . . . . xix

A.4 Stecklinge 11 bis 14 aus dem Nährlösungsversuch. . . . . . . . . . . . . . . . . . xx

A.5 Steckling 15 aus dem Nährlösungsversuch. . . . . . . . . . . . . . . . . . . . xxi

A.6 Stecklinge 3 bis 6 aus dem Leitgewebeversuch. . . . . . . . . . . . . . . . . xxii

A.7 $\quad$ Stecklinge 7 bis 10 aus dem Leitgewebeversuch. . . . . . . . . . . . . . . . . xxiii

A.8 Stecklinge 11 bis 14 aus dem Leitgewebeversuch. . . . . . . . . . . . . . . xxiv

A.9 Stecklinge 15 aus dem Leitgewebeversuch. . . . . . . . . . . . . . . . . . xxv

A.10 Statistische Auswertung für die Modelldaten von Hauptversuch 1. . . . . . . . . xxvi

A.11 Statistische Auswertung für die Zweipunkt-Modelldaten von Hauptversuch 2. xxvii

A.12 Statistische Auswertung für die Dreipunkt-Modelldaten von Hauptversuch 2. xxviii 


\section{Tabellenverzeichnis}

2.1 Zusammenstellung spezifischer elektrischer Widerstände ausgewählter Substrate. 13

$2.2 \quad$ Übersicht von Veröffentlichungen zu verschiedenen Messtechniken. . . . . . . . 25

$3.1 \quad$ Aufschlüsselung der Bezeichnung impedanzspektroskopischer Messungen. . . . . 42

3.2 Zusammenstellung der Versuchsbedingungen für beide Hauptversuche. . . . . . 45

$4.1 \quad$ Statistische Auswertung der Rohdaten von Hauptversuch 1. . . . . . . . . . . . 89

$4.2 \quad$ Statistische Auswertung der Rohdaten von Hauptversuch 2. . . . . . . . . . . . 94 



\section{Abkürzungsverzeichnis}

A (Querschnitts-)Fläche eines Leiters

AC alternating current (Wechselstrom)

AZ Absorptionszone (Wurzelhaarzone)

BHD Brusthöhendurchmesser

BNC -Stecker; spezielle Bauform eines Koaxialsteckers (Bayonet Neill Concelman)

C elektrische Kapazität

DC direct current (Gleichstrom)

DIN Deutsches Insitut für Normung

EIS Elektrochemische Impedanzspektroskopie

EN europäische Norm

F $\quad$ SI-Einheit der elektrischen Kapazität

f Frequenz

FRA frequency response analyzer (Frequenzganganalysator)

GPR Ground Penetrating Radar

H SI-Einheit der Induktivität (Henry)

$\mathbf{H z} \quad$ Hertz

I elektrischer Strom, Stromstärke

i Momentanwert des elektrischen Stroms

$\Im(Z) \quad$ Imaginärteil der Impedanz

K SI-Einheit der (Farb-)temperatur (Kelvin)

kHz Kilohertz

$\mathbf{k} \Omega \quad$ Kiloohm

L elektrische Induktivität 


\section{Abkürzungsverzeichnis}

METLA Metsätutkimuslaitos (Finnisches Institut für Waldforschung)

MHz Megahertz

NPK -Dünger; Dünger, der die Hauptnährelemente Stickstoff, Phosphor und Kalium enthält (Volldünger).

$\phi \quad$ Phasenwinkel

R elektrischer Widerstand

$\Re(Z) \quad$ Realteil der Impedanz

$\mathbf{R}_{\mathbf{E}} \quad$ Erdungswiderstand

REM Rasterelektronenmikroskop

RFA Röntgenfluoreszensanalyse

RG 58 Radio Guide 58 (Typenbezeichnung für koaxiale Kabel)

RG 174 Radio Guide 174 (Typenbezeichnung für koaxiale Kabel)

$\rho \quad$ spezifischer elektrischer Widerstand

$\omega \quad$ Kreisfrequenz

$\Omega \quad$ SI-Einheit des elektrischen Widerstandes $(\mathrm{Ohm})$

$\Omega \mathrm{m} \quad$ SI-Einheit des spezifischen elektrischen Widerstandes (Ohmmeter)

$\mathrm{S} \mathrm{cm}^{-1} \quad$ SI-Einheit der elektrischen Leitfähigkeit

$\tau \quad$ Zeitkonstante

t Zeit

S Summe der Absorptionsfläche (surface)

U Elektrische Spannung

u Momentanwert der elektrischen Spannung

V2A bestimmte Edelstahlsorte

VDE Verband der Elektrotechnik, Elektronik und Informationstechnik

W Watt

Z Impedanz 


\section{Einleitung}

Das Wurzelsystem macht näherungsweise die Hälfte der Biomasse einer Pflanze aus (Mancuso (2011)). Dementsprechend ergeben Untersuchungen, dass die Biomasseproduktion bestimmter Wälder zu 50 \% unter der Erdoberfläche geschieht (Hendrick und Pregitzer (1992)). Die Wurzel bildet so, neben Stamm und Krone, eines der Hauptkompartimente des Kormus einer Pflanze.

Das Wurzelsystem hat jedoch nicht nur aus quantitativem Blickwinkel eine große Bedeutung für das Gesamtsystem Baum, sondern auch auf funktionaler Ebene. So erfüllt das Wurzelsystem neben der Verankerung des Baumes im Boden auch die Funktion, Wasser und darin gelöste Nährstoffe aufzunehmen.

Störungen im Wurzelsystem können in der Folge Standsicherheitsprobleme und mangelnde Resistenz gegenüber Parasiten bewirken (Balder (1998)). Gerade im Hinblick auf den Klimawandel ist ein intaktes Wurzelsystem für die Versorgung des Baumes mit Wasser in länger anhaltenden Trockenperioden eine Grundvoraussetzung, besonders im städtischen Bereich.

Die Analyse des Wurzelsystems einer Pflanze, insbesondere eines Baumes, ist allerdings problematisch. Im Gegensatz zum oberirdischen, sichtbaren Teil (Spross/Stamm und Blätter/Krone) der Pflanze, ist der unterirdische Teil (Wurzelsystem) unsichtbar. Bestimmte Größen und Eigenschaften des Wurzelsystems können aus diesem Grund nicht ermittelt werden. Die „Unsichtbarkeit" dieses lebenswichtigen Grundorgans und die damit verbundenen Messschwierigkeiten legt bereits der Titel eines einschlägigen Fachbuches „Plant roots: the hidden half" (Waisel u. a. (2002)) nahe.

Bisherige Wurzeluntersuchungen (z. B. Ausgraben des Wurzelsystems bzw. von Teilen desselben, in situ Monitoring z. B. durch die sogenannte Profilwandtechnik, experimentelle Untersuchungen z. B. in Wurzelboxen oder Markierungstechniken z. B. durch Radioisotope) sind zeitaufwendig und/oder beschädigen das Wurzelsystem (Polomski u.a. (2002)).

Für die Visualisierung im Bereich der verankernden Grobwurzeln (Durchmesser $>2 \mathrm{~cm}$ ) gibt es einige Untersuchungen aus den letzten Jahren (z. B. Hruska u.a. (1999), Stokes u.a. (2002)). Diese Wurzeln können mit Hilfe eines Bodenradars (GPR) auf bestimmten Standorten sichtbar gemacht werden (Vianden (2013)). Allerdings kann über den Zustand der Wurzeln keine Aussage getroffen werden.

Durchmesserschwächere Wurzeln (Durchmesserklassen $<2 \mathrm{~cm}$ ) können bislang nur mechanisch durch Druckluft freigelegt oder freigespült werden. Besonders Nadezhdina und Čermák (2003) weisen darauf hin, dass sich die „Airspade-Technik“ auch im Bereich der Freilegung der Feinwurzeln bewährt hat. Insbesondere zu den Messmethoden von Fein- und Feinstwurzeln von Bäumen gibt es wenige Untersuchungen und kaum etablierte Messverfahren. Dabei ist die Quantifizierung dieser Wurzeln von grundlegendem Interesse, nicht nur aus wissenschaftlicher Sicht.

Für die zerstörungsfreie Untersuchung des Wurzelsystems, besonders im urbanen Bereich, 


\section{Einleitung}

wurde an der Hochschule für angewandte Wissenschaft und Kunst in Göttingen im Jahr 2007 ein Forschungsschwerpunkt („Städtischer Wurzelraum“) etabliert. Dieser widmet sich besonders drei Themenschwerpunkten. Zum einen sollen geophysikalische Verfahren getestet werden, um das Wurzelsystem zerstörungsfrei zu visualisieren, zum anderen soll der Bodengashaushalt des Stadtbodens untersucht werden. Der dritte Teilbereich nimmt sich der Feinwurzeln eines Baumes und ihrer mengenmäßigen Erfassung an. Der letzte Aspekt ist Schwerpunkt der vorliegenden Arbeit. Die Möglichkeiten und Grenzen dreier elektrischer Verfahren sollen dabei validiert werden:

- Erdungsmessungen (z. B. Yamaura u. a. (2000))

- Bodenwiderstandsmethode (z. B. Aubrecht u. a. (2006)

- Impedanzspektroskopie (z. B. Cao u. a. (2011))

Für die Quantifizierung der Feinwurzeln werden einerseits monofrequente (Erdungsmessung und Bodenwiderstandsmethode), als auch multifrequente Messtechniken (Impedanzspektroskopie) getestet. Diese relativ neuen Methoden sind zerstörungsarm und verhältnismäßig einfach anzuwenden. Elektrische Eigenschaften einer Pflanze, insbesondere die ihres Wurzelsystems, sind jedoch aufgrund der Heterogenität ihres Aufbaus und ihrer Zusammensetzung äußerst komplex. Im Gegensatz zu Festkörpern wie Metallen o. ä. wird die Struktur von Pflanzengeweben nicht durch annähernd regelmäßige Gitterstrukturen bestimmt, sondern vielmehr durch eine Vielzahl verschiedenster Zelltypen und Organe mit unterschiedlichen Eigenschaften und Geometrien.

Elektrische Verfahren, bei denen ein Stromkreis zwischen Baum und Boden angelegt wird, und die Spannung zwischen den Polen gemessen wird, werden in den letzten Jahren durch verschiedene Forschergruppen für die Feinwurzelquantifizierung herangezogen. Allen gleich ist das Bestreben, einen Zusammenhang zwischen elektrischen Größen von Pflanzen (z. B. elektrischer Widerstand und Kapazität) und biologischen Größen (z. B. Feinwurzelmasse oder -oberfläche) zu finden.

Ergebnisse der angesprochenen monofrequenten Verfahren wurden bereits publiziert (z. B. Čermák u. a. (2006), Aubrecht u. a. (2006) oder Fukuma u. a. (2003)). Das Verfahren der Impedanzspektroskopie an Pflanzen wird besonders vom finnischen Institut für Waldforschung (METLA) erprobt, um pflanzenspezifische Größen (u. a. Feinwurzelmasse) zu ermitteln (z. B. Repo u. a. (2005)). Betrachtet man weiterführende Literatur zu diesem Thema, so wird schnell deutlich, dass die Komplexität der biologischen Strukturen auch eine komplexe physikalische Beschreibung des biologischen Systems Pflanze zur Folge hat (Zhang und Willison (1991)).

Die beschriebenen Ergebnisse sind jedoch aus unterschiedlichen Gründen kaum evaluiert bzw. in einem angemessenen Stichprobenumfang verifiziert worden. Auch werden bestimmte Parameter miss- oder kaum beachtet. Speziell bei den Erdungsmessungen ist der Stichprobenumfang sehr gering, auch sind hier mögliche Einflussfaktoren nicht untersucht worden. Die Bodenwiderstandsmethode ist bisher von wenigen Autoren getestet und kritisch evaluiert wor- 
den (Urban u. a. (2011)). In dieser Arbeit wird die Methode vorwiegend modellhaft untersucht, um die grundsätzliche Funktionsweise zu überprüfen.

Ziel dieser Arbeit ist es nun, die genannten Verfahren kritisch zu überprüfen und mögliche sensible Parameter aufzudecken. Die Impedanzspektroskopie wurde in der vorliegenden Arbeit am umfangreichsten getestet, da mit ihr der größtmögliche Erfolg erwartet wurde.

Zunächst wurden schrittweise wichtige Einflussfaktoren nacheinander geprüft, um einen optimalen Versuchsaufbau für weitere Messungen zu planen. Durch den daraus entstandenen Versuchsaufbau und die hier angewendete Messtechnik ist es möglich, eine hohe Stichprobenanzahl und gleichzeitig zeitlich sehr hochauflösend zu messen. So können kleinste Schwankungen der Messwerte über einen längeren Zeitraum erhoben werden, was in den einschlägigen Veröffentlichungen bisher nicht möglich war. Dabei werden physikalische und biologische Daten nahezu zerstörungsfrei erhoben. 



\section{Stand des Wissens}

In diesem Kapitel werden die drei zu testenden Verfahren erläutert, wobei der Fokus auf ihre Unterschiede gelegt wird.

Die Erdungsmessung muss bei der Betrachtung der Verfahren als Erste genannt werden, da sie die Grundlage für die Bodenwiderstandsmethode und die Impedanzspektroskopie bildet. Nach Beschreibung der Bodenwiderstandsmethode wird detaillierter auf die Impedanzspektroskopie an Pflanzen eingegangen.

Zuvor werden jedoch grundlegende Erkenntnisse zu Wurzeln und deren physiologischen Eigenschaften erarbeitet. In diesem Zusammenhang erscheinen das Wurzelwachstum und die Einteilung von Wurzeln anhand ihres Durchmessers relevant. Des Weiteren soll auf den Wassertransport eingegangen werden, da davon ausgegangen wird, dass elektrischer Strom und Wasser (Elektrolyt) über den gleichen Weg fließen.

\subsection{Aufbau und Funktion einer Pflanzenwurzel}

Grundsätzlich werden Pflanzenwurzeln aufgrund ihres Durchmessers in unterschiedliche Kategorien unterteilt. Je nach Wurzeldurchmesser ändert sich auch ihre Funktion. Insbesondere die durchmesserschwächsten Fraktionen des Wurzelsystem sind dabei von besonderer Wichtigkeit, weil hier sowohl das Längenwachstum der Wurzeln, als auch die Wasseraufnahme und der -transport erfolgen.

\subsubsection{Morphologie und Anatomie}

Die Nomenklatur der verschiedenen Typen von Wurzeln variiert in der Literatur. Beispielsweise werden nach Joslin und Henderson (1987) alle Wurzel $<5 \mathrm{~mm}$ als Feinwurzeln bezeichnet, wohingegen Matamala u. a. (2003) Wurzeln $<2 \mathrm{~mm}$ als Feinwurzeln bezeichnen. Vanninen und Mäkelä (1999) unterscheiden weiterhin kleine $(\leq 2 \mathrm{~mm})$ und große Feinwurzeln $(2 \mathrm{~mm}-5 \mathrm{~mm})$ von einander. In dieser Arbeit wird die oft zitierte Einteilung nach Köstler u. a. (1968) zugrunde gelegt. Diese weisen für Wurzelsysteme sechs Typen aus: Starkwurzeln ( $>50 \mathrm{~mm})$, Derbwurzeln $(20 \mathrm{~mm}-50 \mathrm{~mm})$, Grobwurzeln $(5 \mathrm{~mm}-20 \mathrm{~mm})$, Schwachwurzeln $(2 \mathrm{~mm}-5 \mathrm{~mm})$, Feinwurzeln $(1 \mathrm{~mm}-2 \mathrm{~mm})$ und Feinstwurzeln $(<1 \mathrm{~mm})$. Stark-, Derb- und Grobwurzeln (i.e.S.) werden darüber hinaus oftmals allgemein als „Grobwurzeln“ zusammengefasst. Schwach-, Feinwurzeln (i. e. S) und Feinstwurzeln als „Feinwurzeln“.

Während die Grobwurzeln die Pflanze im Boden verankern (Skelettwurzeln), einen sicheren Halt gewährleisten und Nährstoffe speichern, übernehmen vorwiegend die Feinwurzeln die Funktionen des Streckenwachstums, die Erschließung des Bodens und der Aufnahme von Wasser und Nährstoffen (Faserwurzeln und Wurzelhaare) (Polomski und Kuhn (2001)). Durch Verkorkung subepidermaler Zellschichten (nach Absterben der Rhizodermis) bildet sich im 
Laufe der Entwicklung der Feinwurzeln ein Cutisgewebe (Exodermis), das in der Regel eine Wasseraufnahme verhindert. So ist die Wasseraufnahme allein auf die Feinwurzeln (Feinstwurzeln) beschränkt. Hier steht die Pflanze in direktem Austausch mit dem Boden und dem darin befindlichen Bodenwasser.

Grundsätzlich ist der anatomische Aufbau einer Wurzel dem eines Sprosses ähnlich. Dabei unterscheidet sich stammfernes jedoch deutlicher als stammnahes Wurzelholz vom Holz des Sprosses (Müller (2005)). Radial betrachtet besteht die Pflanzenwurzel aus einem inneren Bereich, dem Zentralzylinder, und einem äußeren Bereich, dem Cortex (Wurzelrinde).

Der Zentralzylinder ist für den Nährstoff- und Wassertransport sowie die Weiterleitung in höhere Regionen der Pflanze zuständig. Er besteht aus unterschiedlichen Gefäßtypen (Ring-, Tüpfel- und Schraubengefäßen) und dem ein- oder mehrschichtigem Perikambium (Perizykel) im äußersten Zentralzylinderbereich. Das Perikambium spielt u.a. bei der Entwicklung von Seitenwurzeln eine Rolle. Im Folgenden werden die Seitenwurzeln als Wurzeln zweiter Odnung bezeichnet. Wie im Sprossbereich lässt sich im Zentralzylinder eine Unterscheidung in Xylem und Phloem in variierender Anordnung erkennnen.

Der Zentralzylinder ist von mehrschichtigen, kugeligen Parenchymzellen umgeben (Rindenschicht). Die innere Rindenschicht (Endodermis) besteht aus deutlich kleineren und engeren Parenchymzellen als die des Zentralzylinders. So können Zentralzylinder und Cortex anatomisch gut von einander abgegerenzt werden. Die Endodermis mit dem sogenannten Casparyschen Streifen lässt Wasser und darin gelöste Nährstoffe durch die Zellen dringen, nicht aber durch die Zellwände. Die einschichtige Wurzelrinde (im folgenden Rhizodermis genannt) bildet durch Ausstülpungen die sogenannten Wurzelhaare (siehe dazu Abschnitt 2.1.3).

Für die in dieser Arbeit angestellten Überlegungen nimmt die Wurzelspitze mit ihren Zonierungen eine zentrale Rolle ein. In der Literatur wird sie meist in vier Regionen unterteilt: die Differenzierungszone, die Streckungszone, die Teilungszone und den Bereich der Wurzelhaube (Kalyptra). Die drei erstgenannten Regionen sind vom Zellaufbau betrachtet ähnlich, so dass eine genaue Abgrenzung der Zonen nicht möglich ist (Nabors (2007)). Daher überlappen die Differenzierungszone und die Streckungszone sowie die Streckungszone und die Teilungszone in Abbildung 2.1. Lediglich die Wurzelhaube lässt sich von den anderen Zonen deutlich unterscheiden. Äußerlich erkennbar ist die Differenzierungszone durch die Ausbildung von Wurzelhaaren.

Die einige Millimeter lange Streckungszone schließt zur Peripherie hin betrachtet an die Differenzierungszone an. Die Teilungszone besteht aus meristematischem Gewebe (Apikalmeristem). Die Wurzelhaube wird aus parenchymatischen Zellen gebildet und schützt die embryonalen Zellen des Apikalmeristem wie eine Art Fingerhut. Die Streckungszone, die Teilungszone und die Wurzelhaube sind die am Wurzelwachstum beteiligten Zonen der Wurzeln. 


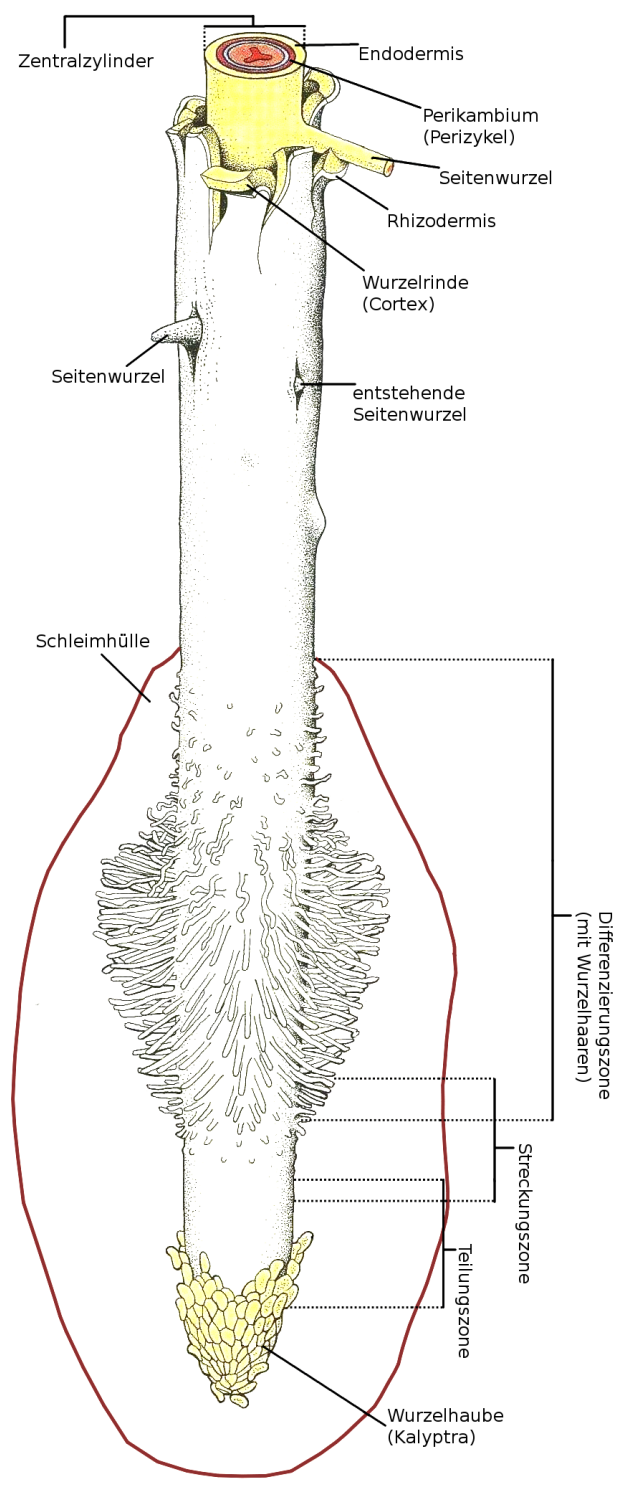

Abbildung 2.1: Schematische Darstellungen einer Wurzelspitze (verändert nach Raven u.a. (2000)).

\subsubsection{Wurzelwachstum}

Das meristematische Gewebe in der Teilungszone der Wurzelspitze bildet neue Zellen durch Zellteilung. Die gebildeten Zellen teilen sich erneut, bis sie in der Streckungszone an Größe zunehmen. Dabei variiert die Zellteilung hinsichtlich Geschwindigkeit und Häufigkeit bei der radialen Betrachtung dieser Zone. Äußere Zellen sind dabei aktiver als zentrale (Doerner (1998)). In der Zellstreckungszone, der eigentlichen Wachstumszone, erfolgt eine Größenzunahme (Streckung) der Zellen fast ausschließlich entlang der Wurzelachse (Längenwachstum). Das Längenwachstum einer Wurzel ist auf diesen Bereich begrenzt. In weiteren Schritten differenzieren sich diese Zellen weiter, bis schließlich eine Unterscheidung in Cortex und Zentralzylinder erkennbar ist.

Das Vorschieben der Wurzel in den Boden bedingt das Ablösen von peripheren Zellen der 
Kalyptra. Diese abgelösten Zellen sowie die jüngeren Parenchymzellen der Wurzelhaube werden von einem schleimigen Sekret aus Polysacchariden und Pektinen umgeben (Schleimhülle). Dieses Sekret wirkt reibungshemmend und erleichtert die Durchwurzelung in das Erdreich (Raven u. a. (2000)).

\subsubsection{Wasseraufnahme und -transport in der Pflanze}

Da die Untersuchungen von der Annahme ausgehen, dass elektrischer Strom bei den zu testenden Verfahren auf ähnliche Weise wie das Wasser in den Baum transportiert wird, ist es notwendig, diesen Weg biologisch genauer zu erläutern.

Aus physikalischer Sicht erfüllt Wasser, durch dessen Fähigkeit Salze zu lösen und ionische Verbindungen zu beherbergen, die Funktion eines Elektrolyten im betrachteten System. Im Wasser gelöste Ionen dienen als Ladungsträger für den Stromtransport. In diesem Kapitel soll lediglich der Saftstrom der Pflanzen mit sekundärem Dickenwachstum betrachtet werden, da sämtliche Untersuchungen und Messungen an solchen durchgeführt wurden. Grundsätzlich müssen beim Transport von Wasser in einer (höheren) Pflanze radiale und axiale Flüsse von einander abgegrenzt werden. Bei der Aufnahme von Wasser über die Wurzelhaare hin zum Zentralzylinder ist dieser Transport zunächst radial. Lösch (2001) beschreibt zwei Kräfte des Wassereinstromes vom Boden in die Pflanze, die osmotisch bedingte Sogwirkung und den Massenfluss von Wasser bedingt durch die Transpiration (Transpirationssog). Im Falle der in dieser Arbeit untersuchten, zunächst blattlosen Stecklinge, besteht kein Transpirationssog.

Die eigentliche Aufnahme des Elektrolyten geschieht über die Wurzelhaarzone. Diese Zone ist in der Regel nur wenige Zentimeter lang und besitzt an dieser Stelle $1 \mathrm{~mm}-2 \mathrm{~mm}$ lange Wurzelhaare (Trichome). Die Lebensdauer dieser Aufnahmeorgane ist auf wenige Tage begrenzt. Wasser kann über zwei, nach Raven u.a. (2000) über drei Wege bis zum Zentralzylinder gelangen, den apoplastischen, den symplastischen und den transmembranen Weg (vgl. Abbildung 2.2.

Beim apoplastischen Transport wird der Weg über die Zellwände, beim symplastischen Transport über die Plasmodesmen (von Protoplast zu Protoplast) und bei dem transmembranen Transport von Zelle zu Zelle (mit Durchquerung der Zellvakuolen) genommen. An der Endodermis läuft der Transportweg symplastisch ab. Die Endodermiszellen sind deutlich anders geformt und angeordnet (siehe Abschnitt 2.1.1). Zudem sind die Wände dieser Zellen verdickt und besonders durch amorphe Polyester aus langkettigen Hydroxy-, Epoxy-, und Oxosäuren mit gesättigten und ungesättigten Mono- und Dicarbonsäuren (im Wesentlichen Suberin) imprägniert. Suberin wirkt lipophil und hydrophob, so dass diese Substanz eine Barriere für den Wassertransport bedeutet (Weiler und Nover (2008)). Bedingt durch seine Anordnung wird dieser Zellverband als Caspary-Streifen bezeichnet (siehe Abbildung 2.2a). Um in den Zentralzylinder zu gelangen, muss das Wasser mehrere Male (je nach Transporttyp) eine Membran passieren. An der Stelle des Casparischen Streifens führt der Weg zwangsweise über Cytoplasma und Vakuole der Durchlasszellen in den Zentralzylinder. Hier werden die Zellmembran und unter Umständen auch der Tonoplast dieser Zellen durchwandert.

Vom Zentralzylinder aus wird Wasser im Xylem der Wurzeln und später im Xylem des 


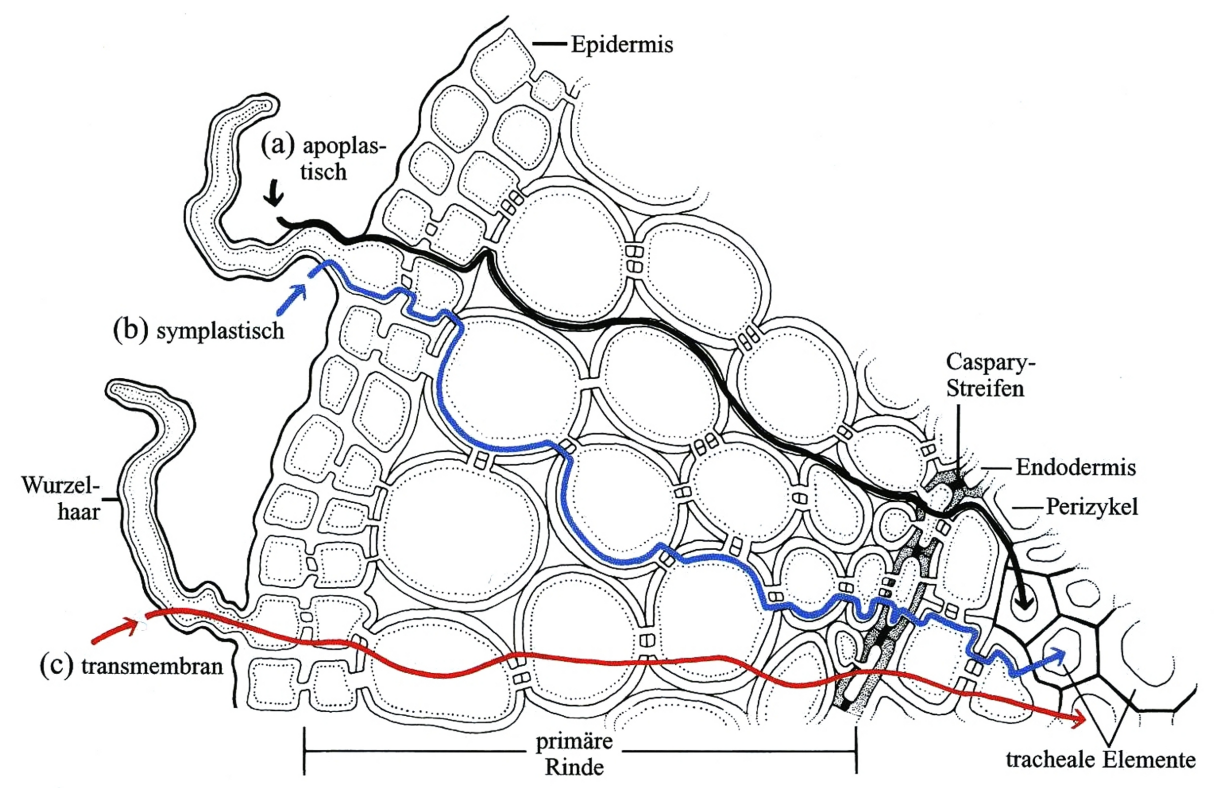

(a)

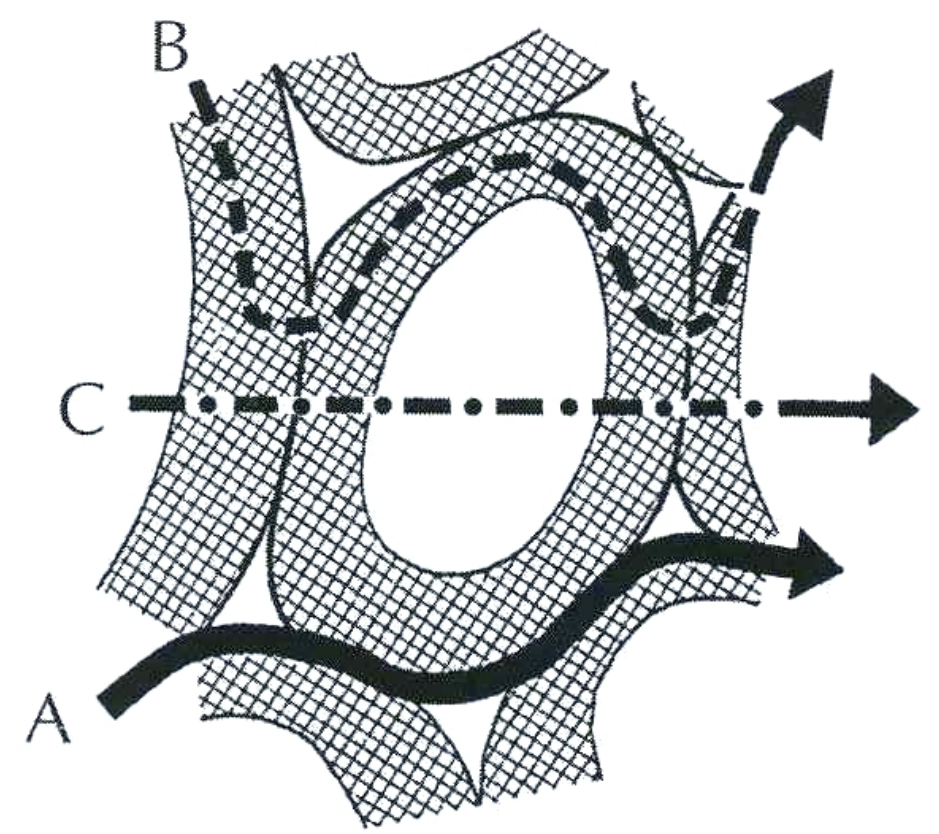

(b)

Abbildung 2.2: Schematische Abbildung des Wassertransports in einer höheren Pflanze (a) gesamter Wassereinstrom vom Wurzelhaar bis in den Zentralzylinder (Raven u. a. (2000) ) und (b) schematische Darstellung der drei Transportwege (A = apoplastischer Transport, $\mathrm{B}=$ symplastischer Transport und $\mathrm{C}=$ transmembraner Transport) auf Zellebene (Schopfer und Brennicke (2010)). 
Stammes axial transportiert. Dieser Übertritt des Wassers aus dem Protoplasten bzw. dem Apoplasten in das Fernleitsystem des Zentralzylinders ist aber bislang nicht hinreichend geklärt (Richter (1997)).

Von der Wurzel bis in das Xylem des Baumes besteht ein durchgängiger, kohärenter Wasserkörper, der es bei Transpiration (nach Ausbildung von Blattorganen) durch einen negativen hydrostatischen Druck zulässt, Wasser durch die Pflanze zu ziehen. Weiterhin wird dies hauptsächlich durch die Kohäsion des Wassers (Wasserstoffbrückenbindungen), die Adhäsion des Wassers an die Zellwände und weitesgehend barrierefreie Tracheiden/Tracheen ermöglicht (Bresinsky u. a. (2008)).

\section{2 Übergangswiderstände}

Übergangswiderstände sind elektrische Widerstände (siehe dazu auch Abschnitt 2.5), die immer dann auftreten, wenn elektrischer Strom von einem elektrisch leitfähigen Material (Leiter) in ein anderes übergeht. In der Elektrotechnik ist man bei Leitungs- oder Kabelübergängen grundsätzlich bestrebt, diese Übergangswiderstände möglichst gering zu halten. Übergangswiderstände sind immer dann besonders hoch, wenn die beiden Leiter aus unterschiedlichen Materialien bestehen bzw. zwischen beiden ungenügender Kontakt herrscht.

Für die im weiteren Verlauf dieser Arbeit untersuchten elektrischen Verfahren wird jeweils ein Stromkreis zwischen Baum (Steckling) und Boden (Nährlösung) mit Hilfe von Edelstahl- bzw. Silberelektroden gebildet (vgl. Abschnitt 3.1. Abschnitt 3.2 und Abschnitt 3.3). Es muss an der Kontaktstelle von stromspeisender Elektrode und Baum (Steckling) und von stromspeisender Elektrode und Boden (Nährlösung) aufgrund der unterschiedlichen Materialien der Leiter mit hohen Übergangswiderständen gerechnet werden. Da bei diesen elektrischen Verfahren aber der elektrische Widerstand zwischen Baum (Steckling) und Boden (Nährlösung) ermittelt werden soll, sind Übergangswiderstände Störgrößen, die den eigentlich interessierenden elektrischen Widerstand verfälschen.

Diese Erhöhung des elektrischen Widerstandes durch Überlagerung von Übergangswiderständen kann durch einen modifizierten Versuchsaufbau umgangen werden. Zum besseren Verständnis der verschiedenen Messweisen und der auftretenden Übergangswiderstände soll das Ersatzschaltbild in Abbildung 2.3 dienen.

Ersatzschaltbilder sind bildliche Darstellungen komplizierter elektrischer Schaltungen in Form leichter berechenbarer und überschaubarer Anordnungen von elektrischen Bauelementen wie bspw. von elektrischen Widerständen (Waloschek (1998)).

Rechts vom Strommesser I befindet sich die Spannungsquelle, U symbolisiert ein Spannungsmessgerät. Die elektrische Spannung kann mit Hilfe des Ohmschen Gesetzes (Gleichung (2.1)) in den elektrischen Widerstand umgerechnet werden. RA, RX und RB symbolisieren verschiedene elektrische Einzelwiderstände.

Die Abbildung zeigt einerseits das Prinzip der Messung, bei denen Übergangswiderstände auftreten und mitgemessen werden (äußerer Messkreis mit gestrichelten Linien) und den modifizierten Versuchsaufbau (innerer Messkreis ohne gestrichelte Linien). Der äußere Kreis 


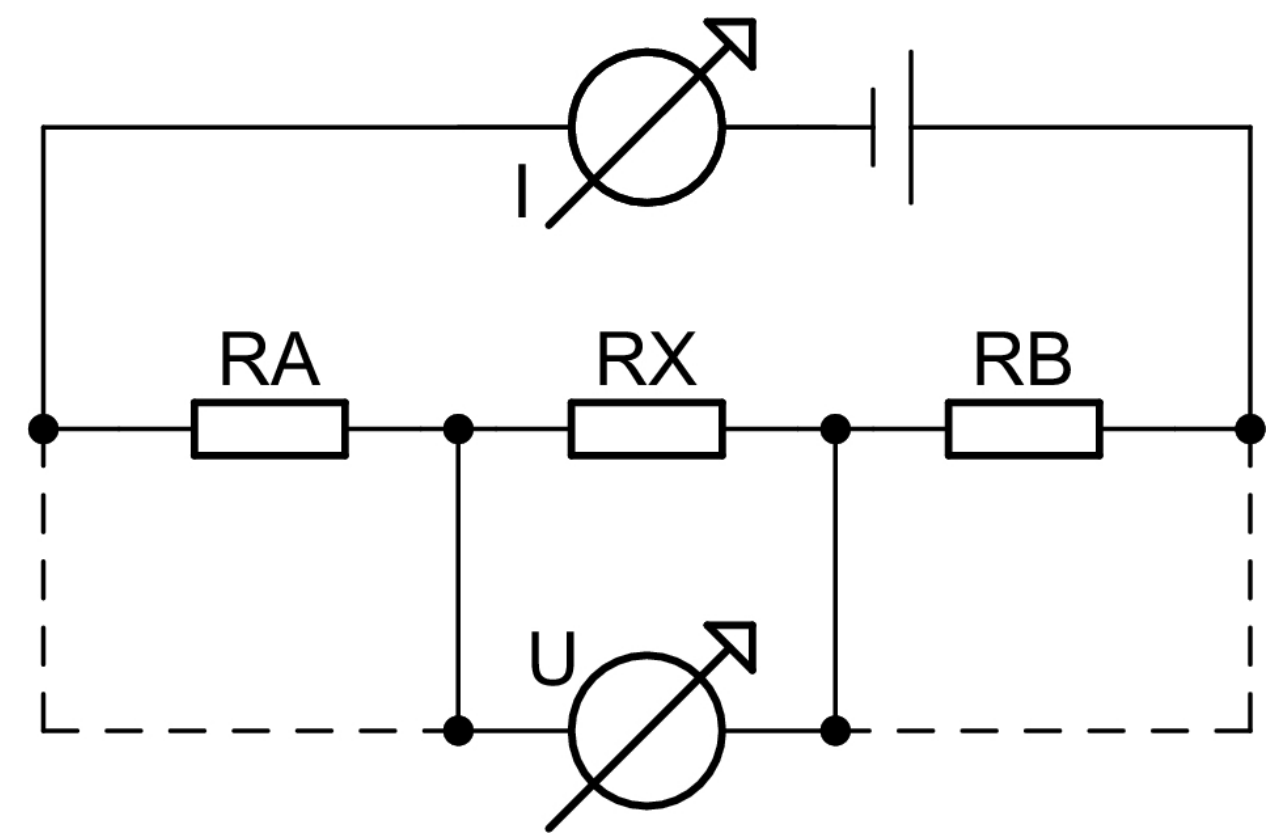

Abbildung 2.3: Ersatzschaltbild für Zwei- und Vierpunktmessungen aus Brasse (2002). Genauere Erläuterungen zu den Bezeichnungen finden sich im Text.

entspricht dem Prinzip der sogenannten Zweipunktmessung (Zweipunktmesstechnik). Bei dieser Methode werden nur zwei Elektroden im Messystem angeschlossen, eine Elektrode erfüllt jeweils die Funktion der Einspeisung des elektrischen Stromes und zugleich der Messung der Spannung zwischen den Elektroden. Im Ersatzschaltbild werden die Übergangswiderstände als RA und RB bezeichnet, der eigentlich interessierende elektrische Widerstand einer Probe mit RX. Wird in dieser Weise gemessen, errechnet sich der Gesamtwiderstand aus der Summe der Einzelwiderstände (RA $+\mathrm{RX}+\mathrm{RB})$.

Der innere Messkreis entspricht einer sogenannten Vierpunktmessung (Vierpunktmesstechnik). Hier sind Speise- und Messkreis voneinander getrennt, so dass zwischen zwei Elektroden ein Stromkreis angelegt wird und dazwischen die Spannung mit zwei weiteren Elektroden gemessen wird (Entkopplung). Übergangswiderstände treten hier zwar an den Speiseelektroden auf, werden aber durch den Einsatz separater Messelektroden nicht mitgemessen (,stromlose Messung").

Messungen in Vierpunktmesstechnik haben den Vorteil, dass der ermittelte Widerstandswert (RX) keine Überlagerung durch Übergangswiderstände (RA und RB) erfährt. Durch diese Entkopplung von Speise- und Messkreis findet ausschließlich der unverfälschte, „wahre“ Wert der Probe Berücksichtigung.

Neben diesen beiden Messvarianten existiert auch die Methode der Dreipunktmessung. Bei dieser Variante werden drei Elektroden kontaktiert. Eine Elektrode erfüllt sowohl Mess-, als auch Speisefunktion, eine andere nur die Speise- und eine weitere nur die Messfunktion. Elektrische Widerstände, die in dieser Art und Weise ermittelt werden, setzen sich folglich aus dem Einzelwiderstand RX und einem Übergangswiderstand (RA oder RB) zusammen.

Dreipunktmessungen finden dort Anwendung, wo der Übergangswiderstand an einer der 
beiden Speiseelektrode aufgrund ausreichenden Kontaktes und ähnlicher Materialien der elektrischen Leiter (Elektrode und Untersuchungsobjekt) zu vernachlässigen ist.

In der einschlägigen Literatur wird für Erdungsmessung die Dreipunktmessung und für die meisten impedanzspektroskopischen Untersuchungen die Zweipunktmessung als Messmethoden genannt.

Bei Messungen im Rahmen von elektrischen Widerstandsmessungen mit Edelstahlelektroden an Prüfkörpern aus Holz hat sich gezeigt, dass die auftretenden Übergangswiderstände ausgesprochen hoch sind und den elekrischen Widerstand der jeweiligen Holzprobe maskieren. Zudem sind die Ergebnisse solcher Zweipunktmessungen nicht reproduzierbar (Hanskötter (2004)).

\subsection{Erdungsmessung}

Grundsätzlich dient ein Erdungssystem dazu, elektrische Ströme bei einem Gewitter ins Erdreich abzuleiten. Auf diese Weise wird ein möglichst geringer elektrischer Widerstand zwischen einem Gebäude und dem Boden erreicht. Der elektrische Strom soll möglichst ungehindert in den Erdboden abgeleitet werden. Die Errichtung eines sogenannten äußeren Blitzschutzsystems wird in der Norm DIN EN 62305-3 (VDE 0185-305) geregelt. Nach diesen Normen darf der Erdungswiderstand nicht mehr als $10 \Omega$ betragen (Kasikci (2013)). Diese Errichtung ist in Deutschland allerdings gesetzlich nicht zwingend vorgeschrieben. So findet sich bspw. in der Niedersächsischen Bauordnung, dass solche baulichen Anlagen mit Blitzschutzanlagen versehen werden müssen, „[...] bei denen nach Lage, Bauart oder Benutzung Blitzschlag leicht eintreten oder zu schweren Folgen führen kann“ (NBauO, §24).

Das äußere Blitzschutzsystem besteht aus einer Fangeinrichtung, Ableitern und einer Erdungsanlage. Die Fangeinrichtungen dienen als Einschlagspunkte für den Blitz, die Ableiter sind elektrisch leitende Verbindungen zwischen der Fangeinrichtung und der Erdungsanlage. Die Erdungsanlage selbst besteht aus elektrischen Leitern (in der Regel leitfähigen Metallen), die in Kontakt mit dem Erdboden stehen, den sogenannten Erdern (Laasch und Laasch (2013)).

Um die Wirksamkeit einer Blitzschutzanlage zu prüfen, werden üblicherweise Erdungsmessungen durchgeführt. Bei klassischen Erdungsmessungen, wie sie im Bereich von baulichen Anlagen durchgeführt werden, wird zwischen Ableitung und Boden ein niederfrequenter Wechselstrom (12 V bei $50 \mathrm{~Hz}$ ) angelegt. Aus dem Quotienten des sich aufbauenden Spannungspotentials $(U)$ und dem eingespeisten Strom $(I)$ errechnet sich nach dem Ohmschen Gesetz der elektrische Widerstand, bzw. der Erdungswiderstand $\left(R_{E}\right)$ (siehe Gleichung (2.1)):

$$
R_{E}=\frac{U}{I}
$$

Der Erdungswiderstand setzt sich typischerweise aus unterschiedlichen Einzelwiderständen zusammen. Auf die genauere Beschreibung eines solchen komplexen Schaltkreises wird in Abschnitt 2.5 eingegangen. Bei Messungen an Bäumen hat sich gezeigt, dass die elektrischen Widerstände der beteiligten Messobjekte im Versuchsaufbau nicht nur mit sogenannten ohmschen Widerständen beschrieben werden können. Neben diesen spielen auch Kapazitäten eine 
entscheidende Rolle (Abschnitt 2.5).

Vorläufig können aber in diesem Messaufbau der Eigenwiderstand des Ableiters, der Übergangswiderstand zwischen Ableiter und Substrat, der elektrische Widerstand des Substrates und die Übergangswiderstände an den (Speise-) Elektroden genannt werden. Da der elektrische Widerstand des Ableiters aus gut leitfähigen Metallen (Edelstahl) besteht, können dieser und der Übergangswiderstand vom Ableiter zur Elektrode vernachlässigt werden. Die wesentlichen Faktoren, die den Erdungswiderstand beeinflussen, sind folglich der Übergangswiderstand zwischen Ableiter und Substrat und das Substrat selbst. Da es einen physikalischen Zusammenhang zwischen der Oberfläche eines Leiters und seinem elektrischen Widerstand gibt, sinkt der Erdungswiderstand gemäß Gleichung $(2.2)$ bei Erhöhung der Oberfläche ab:

$$
R_{E}=\rho \frac{l}{A}
$$

Der spezifische elektrische Widerstand $(\rho)$ ist materialspezifisch und wird daher auch als Materialkonstante bezeichnet. Wird die Oberfläche $(A)$ eines elektrischen Leiters größer, nimmt der elektrische Widerstand insgesamt ab. Umgekehrt nimmt der elektrische Widerstand zu, wenn die Länge (l) zunimmt. Der elektrische bzw. spezifische elektrische Widerstand des Substrates, das der Erder kontaktiert, ist hauptsächlich abhängig vom Wasser- und Ionengehalt des Substrates. Daher haben Bodenarten, die einen hohen Anteil von Fein- und Mittelporen und damit hohe Wasserspeicherkapazitäten aufweisen (z. B. Lehmböden) niedrigere spezifische elektrische Widerstände, als solche mit einem hohem Anteil von (luftgefüllten) Grobporen (z. B. Sande und Sandsteine) bzw. mit einem hohen Skelettgehalt. Dieser elektrische Widerstand ist sehr variabel und kann durch eine separate Messung, die lediglich das Erdreich einbezieht, mit Hilfe eines Erdungsmessgerätes ermittelt werden (Erdwiderstand). Tabelle 2.1 dient der Einschätzung des Erdwiderstandes und zeigt die Variabilität dieses Messparameters.

Tabelle 2.1: Zusammenstellung spezifischer elektrischer Widerstände ausgewählter Substrate (verändert nach Weidelt (2005)).

\begin{tabular}{lll}
\hline Substrat & $\rho$ in $\Omega \mathrm{m}(\min )$ & $\rho$ in $\Omega \mathrm{m}(\max )$ \\
\hline Ton & 3 & $>1000$ \\
Sand & 50 & $>10^{4}$ \\
Schluff & 20 & 50 \\
Lösslehm & 30 & 100 \\
Sandstein & $<50$ & $>10^{5}$ \\
Moorböden & 10 & 150 \\
Natürliche Wässer & 10 & 300 \\
Destilliertes Wasser & & $>10^{3}$ \\
\hline
\end{tabular}


Dadurch, dass die Pflanze mit ihren Feinwurzeln Wasser mit darin gelösten Nährstoffen über den Boden aufnimmt, befindet sie sich hier im direkten Kontakt zum Bodenwasser. Die Wurzelhaare der Wurzelspitzen erfüllen dabei eine Schlüsselfunktion (siehe Abschnitt 2.1.3).

Aus physikalischer Sicht erfüllt der Baum mit seinem Wurzelwerk, genauer gesagt mit den sich im Austausch mit dem Substrat befindlichen Feinstwurzeln, die Funktion eines Erders (Yamada u.a. (2003)). Fast ausschließlich an diesen Stellen ist die Aufnahme von Wasser und darin gelösten Ionen und damit der Transport von elektrischem Strom möglich. Intakte, unbeschädigte Grob-, Derb- oder Starkwurzeln sind durch ihre Wurzelrinde elektrisch isoliert (Čermák u. a. (2006)) und tragen somit nicht zur Erdung des Systems Baum bei. Weist eine Pflanze eine hohe Feinwurzelmasse auf, so ist sie gegenüber einer anderen mit einer geringeren Feinwurzelmasse besser geerdet. Im Falle einer solchen Messung wird der Erdungswiderstand geringer ausfallen.

Einige Arbeiten aus den letzten zwei Jahrzehnten beschäftigen sich mit Erdungswiderständen von Bäumen. Es werden hier bereits Ersatzschaltbilder sowohl für eine Dreipunkt-, als auch eine Vierpunktmessung definiert (Yamaura u. a. (2000)). Es wird ebenso der Hinweis gegeben, dass der Erdungswiderstand gemessen in Vierpunktmessung deutliche Vorteile gegenüber (klassischen) Dreipunktmessungen hat. Zudem werden in einigen Arbeiten Feldstudien in verschiedenen Ländern mit unterschiedlichen Bodenverhältnissen und Baumarten vorgestellt. Erdungswiderstände wurden unter anderem an Zelkoven, Kirschen und Schirmakazien (botanisch nicht genauer definiert) mit Durchmessern von $54 \mathrm{~cm}-64 \mathrm{~cm}$ unter niederfrequenten Bedingungen $(1 \mathrm{~Hz})$ durchgeführt. Hier ergaben sich Erdungswiderstände von $60 \Omega-100 \Omega$. Des Weiteren wird darauf hingewiesen, dass die Vergleichbarkeit von Bäumen untereinander beispielsweise in verschieden Ländern/Regionen Schwierigkeiten bereitet, da der elektrische Widerstand des Bodens einen wesentlichen Einfluss auf den Erdungswiderstand hat (Yamaura u. a. (2002)). Dabei wirkt sich besonders der Wassergehalt des Bodens auf den Erdungswiderstand aus (Yamaura u.a. (2006)).

Erdungsmessungen an Chinesischem Wacholder (Juniperus chinensis) und Walnuss (Juglans regia) über mehrere Wochen zeigen einen deutlichen und sprunghaften Abfall nach einem Niederschlagsereignis. Ein direkter Zusammenhang zwischen Größen des Wurzelsystems und dem elektrischen Widerstand wurden hier nicht untersucht. Auch ist die Stichprobe der untersuchten Bäume gering. Es werden vorwiegend examplarische Einzelbäume, teilweise über einen längeren Zeitraum gemessen (Yamaura u.a. (2006), Yamada u. a. (2003))

\subsection{Bodenwiderstandsmethode}

Die Bodenwiderstandsmethode kann als Weiterentwicklung der Erdungsmessung in zwei Richtungen verstanden werden.

Erstens wird aus physikalischen, mathematischen Gesetzmäßigkeiten eine Formel zur Berechnung der Oberfläche der Wurzelspitze, der sogenannten Absorptionszone (AZ), abgeleitet. Diese Region entspricht der Differenzierungszone mit den Wurzelhaaren (Wurzelhaarzone) einer Pflanze (siehe Abbildung 2.1). Der Erdungswiderstand (siehe Abschnitt 2.3) wird als 
Hauptgröße besonders betrachtet und findet in Gleichung (2.3) Berücksichtigung. Die Formel für die Berechnung der $\mathrm{AZ}$ wird definiert als:

$$
S=\rho L \frac{I}{U}
$$

Wobei $S$ die Summe der Absorptionsflächen (surface) eines Baumes bedeutet. $\rho$ beeinhaltet den spezifischen elektrischen Widerstand des Leitgewebes des Baumes, der durch eine separate Messung in Vierpunktmesstechnik (siehe Abschnitt 2.2) am Stamm gemessen wird (Aubrecht u. a. (2006)).

Zweitens findet der Abstand des Stammes $(L)$ zu einer sogenannten Hilfselektrode Berücksichtigung. I bezeichnet die Stromstärke. Der Strom wird bei dieser Messtechnik mit einer Frequenz von $500 \mathrm{~Hz}$ eingespeist. Die Spannungsdifferenz $(U)$ als ermittelter Parameter zwischen den Messpolen von Baum und Boden findet besondere Berücksichtigung. Letztgenannte Größen, dem Quotienten aus Stromstärke und Spannung, entsprechen dem Erdungswiderstand (vgl. auch Gleichung (2.1).

Bei Versuchen mit 350 Nadel- und Laubbäumen in unterschiedlichen Experimenten wurden starke Zusammenhänge zwischen der Grundfläche und der mit Hilfe der Gleichung (2.3) errechneten Fläche der Absorptionszone (AZ) der Bäume entdeckt. Aus praktischen Gründen war es nicht möglich, die Größe des gesamten Feinwurzelwerkes eines Baumes zu ermitteln und darüber hinaus eine geeignete Stichprobengröße dieser Bäume zu erhalten. Die entwickelte Formel konnte aus diesen Gründen lediglich indirekt verifiziert werden. In einem von Čermák u. a. (2006) beschriebenen Versuch wurde die Hälfte des Wurzelsystems von sieben Fichten (Picea abies), an denen zuvor Bodenwiderstandsmessungen durchgeführt, abgegraben. Nach einer erneuten Messung an diesen Fichten wurde festgestellt, dass sich die errechnete absorbierende Fläche signifikant verkleinerte.

Darüber hinaus wird in dieser Arbeit eine Abschätzung der horizontalen Ausbreitung des Wurzelsystems gegeben. Dies ist über radiale Messungen und der für jede azimutale Messung ermittelten „Potentialcharakteristik“ möglich. Für diese Messungen werden Daten entlang von 4-8 radial vom Stamm wegführenden Linien ermittelt. Die genaue Anordnung der Messelektrode M im Baum und der Hilfselektrode(n) B im Boden soll durch eine vorhergehende Messung ermittelt werden.

\subsection{Impedanzspektroskopie und kapazitive Messverfahren}

Die Impedanzspektroskopie ist im Gegensatz zu den vorher beschriebenen Verfahren eine multifrequente Messtechnik, wobei der Versuchsaufbau dem der Erdungsmessung sehr ähnlich ist.

Im Gegensatz zur Erdungsmessung wird jedoch eine Wechselspannung, im Allgemeinen eine sinusförmige Spannung, an einem Messobjekt mittels zweier Elektroden bei sukzessiv ansteigender bzw. abfallender Frequenz angelegt und ein sinusförmiges Stromsignal gemessen. Aus diesen Größen kann die sogenannte Impedanz (Scheinwiderstand oder komplexer Widerstand) errechnet werden. Die Definition der Impedanz wird im weiteren Verlauf dieses Abschnitts genauer erläutert. 
Die Frequenz $(f)$ in der Maßeinheit Hertz $(\mathrm{Hz})$ gibt die Anzahl dieser sinusförmigen Schwingungen pro Sekunde an. Messungen von Impedanzen bei sukzessiv ansteigenden Frequenzen (Frequenzdurchlauf, Frequenzband) werden in der Literatur synonym als sweep $(s)$ bezeichnet.

Die Wellenbewegungen der sinusförmigen Schwingungen von angelegter Spannung und resultierendem Strom können als zeitlicher Verlauf dargestellt werden, häufig werden jedoch die Wellenbewegung in Kreisbewegungen mit der Kreisfrequenz $\omega$ transformiert. Die Umrechnung der Frequenz $(f)$ in die Kreisfrequenz $(\omega)$ ergibt sich aus:

$$
\omega=2 \pi f
$$

Ein Kreisdurchlauf entspricht also gemäß Gleichung 2.4 zwei sinusförmigen Wellenbewegungen. Die Amplituden der Schwingungen der angelegten Spannung $(U)$ und des resultierenden Stromsignals $(I)$ werden häufig als Pfeile dargestellt. Abbildung 2.4 veranschaulicht die Transformation von der zeitlichen Darstellung (links) in die Kreisdarstellung mit Zeigern (rechts). Auf der Abszisse bzw. bei der Transformation in die Kreisbewegung ist daher sowohl die Zeit $(t)$, als auch die Kreisfrequenz $(\omega)$ dargestellt. Die Transformation in die Kreisdarstellung ist hier lediglich durch einen (annähernden) Halbkreis skizziert. Auf der Ordinate (linker Bildbereich) ist durch die Kleinbuchstaben von Spannung $(u)$ und Strom $(i)$ angedeutet, dass es sich bei dieser Achse um sogenannte Momentanwerte (Augenblickswerte) handelt. Die beiden Größen sind aufgrund der sich ständig ändernden Werte nie konstant.
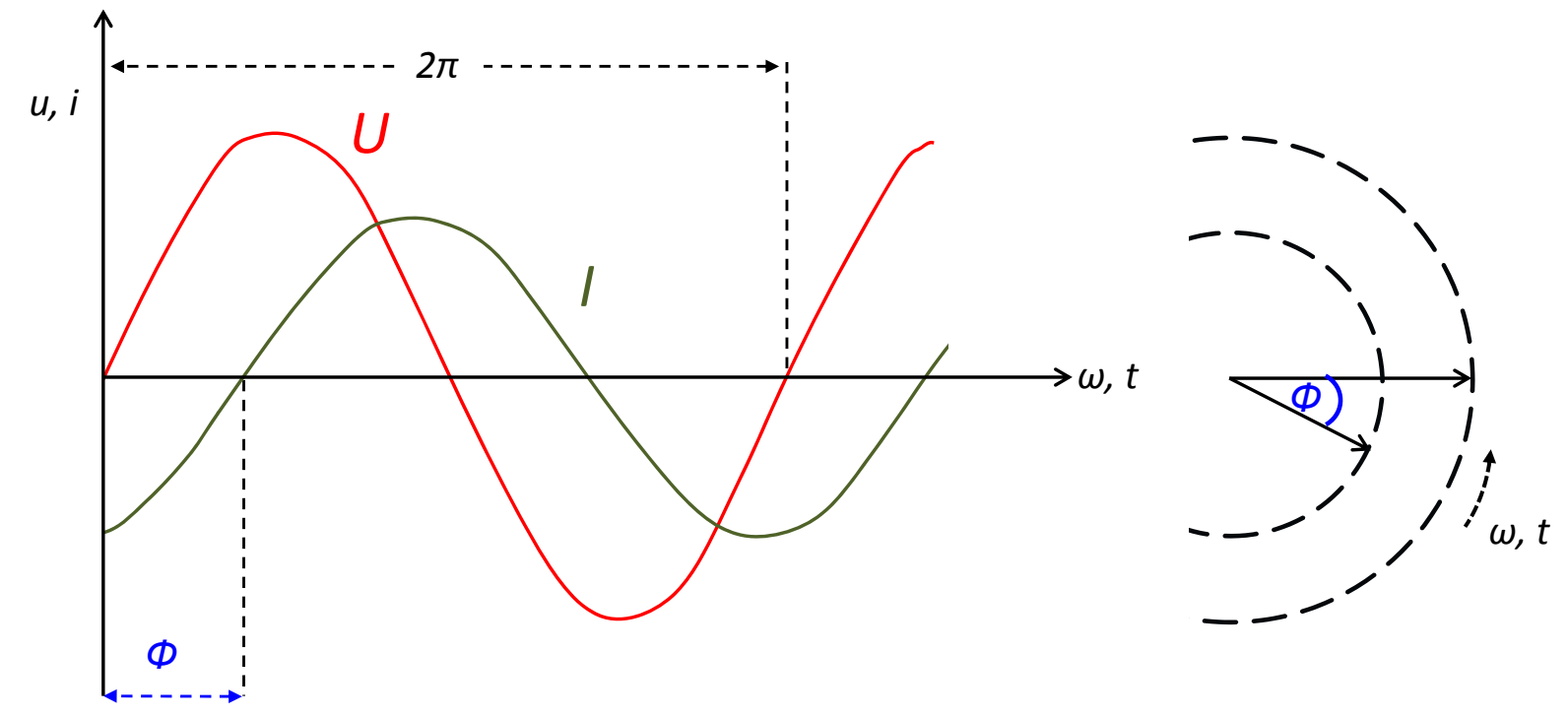

Abbildung 2.4: Darstellung der sinusförmigen Schwingungen von angelegter Spannung $(U)$ und resultierendem Stromsignal ( $I$ ) als zeitlichen Verlauf (links) und nach Transformation in die Kreisdarstellung mit Zeigern (rechts). Weitere Erläuterungen zu dieser Abbildung finden sich im Text. 
Die Schwingungen von Spannung und Strom sind häufig durch einen zeitlichen Versatz gekennzeichnet, der als Phasenverschiebung bezeichnet wird. Diese Phasenverschiebung wird durch den sogenannten Phasenwinkel $(\phi)$ ausgedrückt, der sich auf der Abzisse als (zeitlicher) Versatz der beiden Wellen (links) und als Winkel zwischen Strom und Spannungspfeil in der Kreisdarstellung zeigt (vgl. Abbildung 2.4).

Mit Hilfe der im weiteren Verlauf dieser Arbeit (Abschnitt 3.3.1) beschriebenen Messtechnik können die Impedanz und der Phasenwinkel bei verschiedenen Frequenzen ermittelt werden.

Die Impedanz gibt das Verhältnis von Spannung und Strom an. Mit der Impedanz und dem Phasenwinkel erfolgen weitere Berechnungen.

Trägt man die gemessene Impedanz über der Frequenz auf, so erhält man ein sogenanntes Impedanzspektrum. Üblicherweise beschäftigt man sich mit dem niederfrequenten Bereich von einigen Hertz bis zum hochfrequenten von wenigen Megahertz. Vielfach ist aber nur ein enger Bereich im Gesamtspektrum von Interesse (Zimmermann (2011)).

Zur Vereinfachung der Berechnungen bedient man sich in der Wechselstromlehre komplexer Zahlen (Gauß'sche Zahlen). Abbildung 2.5 zeigt in der komplexen Ebene, wie Impedanz $(Z)$ und Phasenwinkel $(\phi)$ mit dem Realteil der Impedanz $(R)$ und dem Imaginärteil der Impedanz $(j X)$ zusammenhängen. In dieser Abbildung werden ähnlich der Abbildung 2.4 der Phasenwinkel $(\phi)$ und die Impedanz als Zeiger dargestellt. Angedeutet ist auch hier ein Kreis mit der Kreisfrequenz $(\omega)$ (bzw. mit der Zeit $(t)$ ), der seinen Mittelpunkt beim Ursprung zweier Achsen eines kartesischen Koordinatensystems hat. Die Abszisse stellt hier die reelle, die Ordinate die imaginäre Achse dar. Mit Hilfe von Winkelfunktionen können der Realteil der Impedanz und der Imaginärteil der Impedanz aus der Impedanz und dem Phasenwinkel errechnet werden.

Die Impedanz Z wird folgendermaßen definiert:

$$
Z=Z \cos \phi+j Z \sin \phi
$$

Bzw. mit den direkten Bezeichnungen aus Abbildung 2.5

$$
Z=R+j X
$$

Dabei stehen $Z \cos \phi$ bzw. $R$ für den Realteil der Impedanz und $j Z \sin \phi$ bzw. $j X$ für den Imaginärteil der Impedanz mit der imaginären Einheit $j$.

In der Literatur findet man für den Realteil der Impedanz auch die Begriffe Wirkwiderstand oder Resistanz und für den Imaginärteil der Impedanz die Begriffe Blindwiderstand oder Reaktanz. In dieser Arbeit werden die Begriffe Realteil (der Impedanz) bzw. die Kürzel $\Re(Z)$ und Imaginärteil (der Impedanz) bzw. die Kürzel $\Im(Z)$ verwendet.

Phasenverschiebungen treten dann auf, wenn bei zu untersuchenden Messobjekten neben reinen ohmschen auch Wechselstromwiderstände vorkommen, also induktive oder kapazitive Widerstände in Form von Kondensatoren und Spulen. 


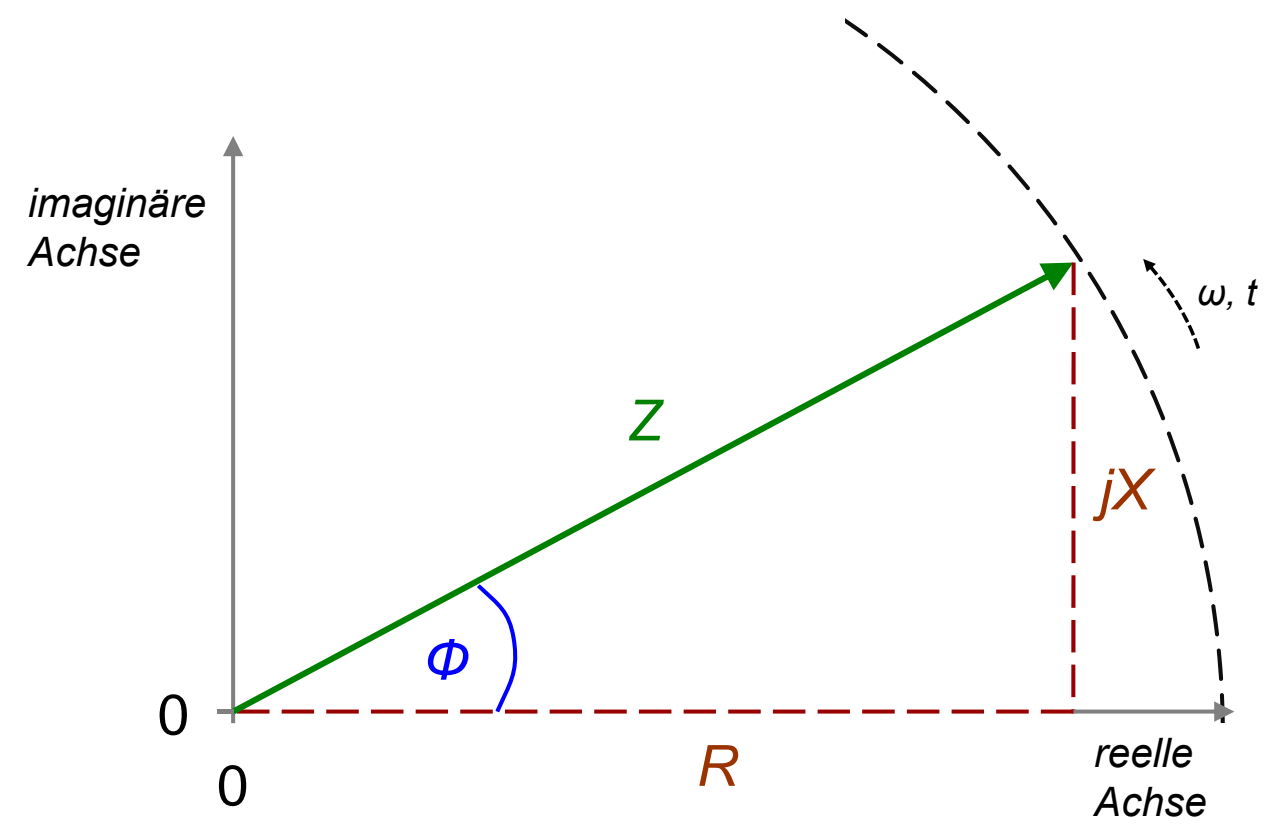

Abbildung 2.5: Darstellung von Impedanz $(Z)$ und Phasenwinkel $(\phi)$ in der komplexen Ebene als Zeigerdiagramm. Die Absizze entspricht der reellen Achse, auf dem der Realteil der Impedanz $(R)$ abgebildet ist, die Ordinate entspricht der imaginären Achse auf dem der Imaginärteil der Impedanz $(j X)$ abgebildet ist.

Wird eine impedanzspektroskopische Untersuchung an rein ohmschen Widerständen durchgeführt, tritt im Frequenzgang keine Phasenverschiebung auf. Betrachtet man eine Messanordnung mit rein ohmschen Widerständen im Impedanzspektrum, so gilt:

$$
Z=R
$$

Die Impedanz $(Z)$ entspricht folglich dem Realteil der Impedanz $(R)$, da der Phasenwinkel $0^{\circ}$ beträgt und damit der Imaginärteil der Impedanz gemäß Gleichung 2.5 0 beträgt. Die Scheitelpunkte der sinusförmigen Schwingungen von Spannung und Strom werden zur gleichen Zeit erreicht, sie sind „in Phase“. Umgekehrt ist bei Vorliegen von reinen Blindwiderständen (Kapazitäten und Induktivitäten) der Realteil der Impedanz zu vernachlässigen. Es gilt folglich:

$$
Z=j X
$$

Die Schwingungen von Spannung und Strom verlaufen bei steigender Frequenz zeitlich versetzt (vgl. Abbildung 2.4). Dabei wird bei einem angelegten Kondensator mit einer Wechselspannung zunehmender Frequenz der Imaginärteil der Impedanz sinken, während bei einer Spule der Imaginärteil der Impedanz steigt. Der Grund dafür ist, dass bei der Spule der Strom der Spannung hinterher eilt, während beim Kondensator der Strom der Spannung voraus eilt. Aus diesem Grund nimmt der Phasenwinkel bei elektrischen Widerstandsmessungen an Spulen positive, bei elektrischen Widerstandsmessungen an Kondensatoren negative Werte an. 
Mathematisch wird die Impedanz bei Kondensatoren folgendermaßen definiert:

$$
Z=\frac{1}{j \omega C}
$$

Dabei stellt $C$ die Kapazität dar, die in der Einheit Farad $(F)$ angegeben wird. Kapazitäten enstehen bei Kondensatoren durch ihre spezielle Bauweise, bestehend aus zwei, sich nicht berührenden Elektroden. Zwischen diesen Elektroden muss es einen nicht leitfähigen Bereich geben (Dielektrikum). Bei einer angelegten Spannung kommt es bei einer der Elektroden zur positiven, bei der anderen zu einer negativen Aufladung. Als Folge davon entsteht ein elektrisches Feld. Durch Kondensatoren kann auf diese Weise elektrische Energie gespeichert werden (Aufladen des Kondensators).

Die Impedanz bei einer Spule als weiteres Bauelement in der Elektrotechnik wird mit folgender Gleichung beschrieben:

$$
Z=j \omega L
$$

$L$ bezeichnet die Induktivität, die in der Einheit Henry $(H)$ angegeben wird. Bei Spulen entsteht durch ihre spezielle Bauweise (Windungen) ein Magnetfeld, durch das eine Spannung erzeugt wird. Das Maß für diese selbstinduzierte Spannung ist die Induktivität.

Bei einer Parallelschaltung aus ohmschem Widerstand $(R)$ und Kondensator $(C)$ erhält man ein RC-Glied oder ein RC-Element mit der Impedanz:

$$
Z=\frac{1}{\frac{1}{R}+j \omega C}=\frac{R}{1+j \omega R C}
$$

In der Literatur wird vielfach die Zeitkonstante $\tau$ zur Interpretation herangezogen, die ein Maß für die Schnelligkeit des Aufladens eines Kondensators darstellt. $\tau$ ergibt sich aus dem Produkt von ohmschen Widerstand und Kapazität:

$$
\tau=R C
$$

Die Betrachtung von sogenannten RC-Gliedern soll an dieser Stelle erfolgen, da in Pflanzensystemen Biomembranen vorkommen, die mit Hilfe von RC-Gliedern beschrieben werden können (Zhang und Willison (1991).

Die Bedeutung der Impedanzspektroskopie für naturwissenschaftliche Fachrichtungen, insbesondere für die Medizin, wurde bereits früh erkannt (z. B. Schwan (1963) und frühere Veröffentlichungen) und wird bis heute vor allem in der Materialforschung und für biologische Fragestellungen an tierischen Zellen (z. B. ex vivo an Lungen von Ratten etc.) erprobt (Dean u. a. (2008)).

Mit Hilfe der Impedanzspektroskopie können bspw. Transportvorgänge in biologischen Systemen oder Grenzflächen von Materialien in situ analysiert werden.

Das Ziel bei der Impedanzspektroskopie ist es, den Betrag der Gesamtimpedanz genauer zu analysieren und die unbekannten einzelnen Komponenten mit Werten zu belegen. 
Trägt man den Real- und Imaginärteil gegeneinander auf, so erhält man einen charakteristischen Kurvenverlauf, eine Art Fingerabdruck für das zu analysierende Material oder System.

Bei der Interpretation dieses charakteristischen Datensatzes werden Ersatzschaltbilder zur Hilfe genommen, die auf geeigneten biologisch-physikalischen Modellen basieren. Ziel ist die möglichst genaue Beschreibung der Werte durch die verschiedenen Impedanzelemente (Ende und Mangold (1993)). Dabei ist das Ergründen des geeigneten Modells von besonderer Wichtigkeit bzw. Schwierigkeit, da die Anpassung des charakteristischen Impedanzspektrums an das Ersatzschaltbild in der Regel nicht eindeutig ist. Vielmehr existiert eine Vielzahl von Modellen und damit eine Vielzahl von Ersatzschaltbildern, die die Daten aus der EIS (Elektrochemischen Impedanzspektroskopie) mehr oder weniger gut anpasst (MacDonald (1987)). Daher sollte man grundsätzlich eine Vorstellung von dem Weg des Stromes und dessen Wechselwirkung mit dem Objekt haben. Erst durch die biologischen Kenntnisse kann die Entwicklung eines vollständigen und adäquaten Modells durchgeführt werden.

Abbildung 2.6 zeigt schematisch den Ablauf dieser theoretischen Grundlagenuntersuchung auf. Im ersten Schritt wird nach der Gewinnung impedanzspektroskopischer Daten am Messobjekt nach Modellen bzw. Ersatzschaltbildern gesucht, die die gemessenen impedanzspektroskopischen Daten möglichst genau erklären. In der zweiten Phase erfolgt die qualitative Beurteilung des Modells. Hier soll eine möglichst enge Beziehung zwischen Objektaufbau bzw. den entsprechenden Ersatzschaltbildern und den gefundenen Modellen vorhanden sein. Im letzten Schritt erfolgt die quantitative Zuordnung der Objektparameter zu den Modellparametern. Die so ermittelten Schätzwerte der Modellparameter werden zur weiteren statistischen Auswertung herangezogen. Einen umfassenden Überblick über die Methode der Impedanzspektroskopie findet sich bei MacDonald (1987) sowie bei Barsoukov und MacDonald (2005).

Im komplexen (biologischen) System Pflanze mit verschiedenen Gewebe- und Gefäßtypen und einer Vielzahl an Zellen ist besonders die Betrachtung des Imaginärteils der Impedanz interessant, da sich biologische Membranen physikalisch als Kondensatoren darstellen.

Die Grundlagen für die physikalische Beschreibung biologischer Strukturen bieten einige zum Teil ältere Arbeiten (Paszewski u.a. (2006), Hayden u.a. (1969), MacDougall u.a. (1987)). Wissenschaftlich untersucht wurden hier die kleinsten Einheiten eines Organismus - die Zelle bzw. Zellverbände. In diesen Arbeiten werden jeweils unterschiedliche physikalische Modelle (Ersatzschaltbilder) für Pflanzenzellen definiert, welche dem Impedanzspektrum einer Messung an Zellen sowohl hinsichtlich Real- wie auch hinsichtlich des Imaginärteils möglichst gut entsprechen. Allen gemein ist die Verwendung von ohmschen Widerständen $(R)$ und Kapazitäten $(C)$ für die Modellierung.

Zhang und Willison (1991) prüften die angesprochenen unterschiedlichen Modelle für Pflanzenzellen auf ihre Genauigkeit und wandten diese und ein eigenes auf selbst erhobene Impedanzspektren von Tomaten- und Karottenzellen an. Hier zeigte sich, dass die beste Übereinstimmung von Mess- und Modelldaten bei der Verwendung eines sogenannten double shell models gibt. 


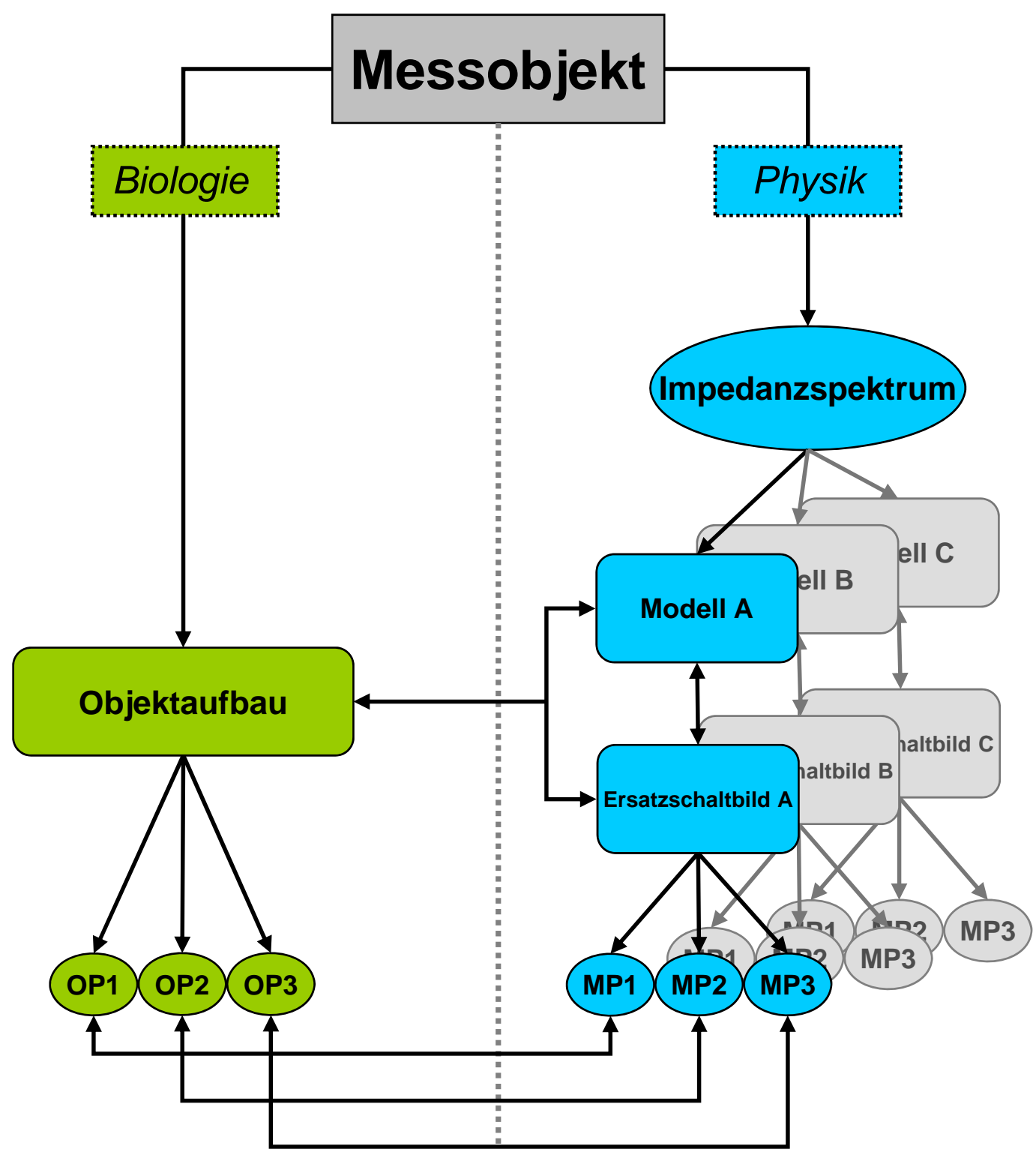

Abbildung 2.6: Veranschaulichung des Vorgehens bei der Modellanpassung nach Gewinnung impedanzspektroskopischer Daten. Begriffserklärung: $\operatorname{MP}(1,2,3)=$ Modellparameter $(1,2,3) ; \mathrm{OP}(1,2,3)=$ Objektparameter $(1,2,3)$. 
Wie in Abbildung 2.7 ersichtlich ist, geht man bei dem Modell von drei ohmschen Widerständen (R1, R2 und R3) und zwei Kondensatoren (C1 und C2) aus. Diese werden bestimmten Zellorganellen einer Pflanzenzelle zugeordnet.

Ohmsche Widerstände erklären zum einen den elektrischen Widerstand des extrazellulären Bereiches, zum anderen den elektrischen Widerstand der Zellsaftvakuole. Der dritte ohmsche Widerstand soll das Cytoplasma modellieren. Die Kondensatoren im Modell entsprechen Biomembranen, wie sie einerseits im Tonoplasten und andererseits in der Plasmamembran vorliegen. Dieses Ersatzschaltbild kann somit als physikalisches Grundmodell betrachtet werden. Bei größeren Zellverbänden bzw. Pflanzenorganen bleibt dieses Schalenmodell in seiner Grundstruktur erhalten. Die absoluten Werte ändern sich je nach Größe und Art der Zellverbände.

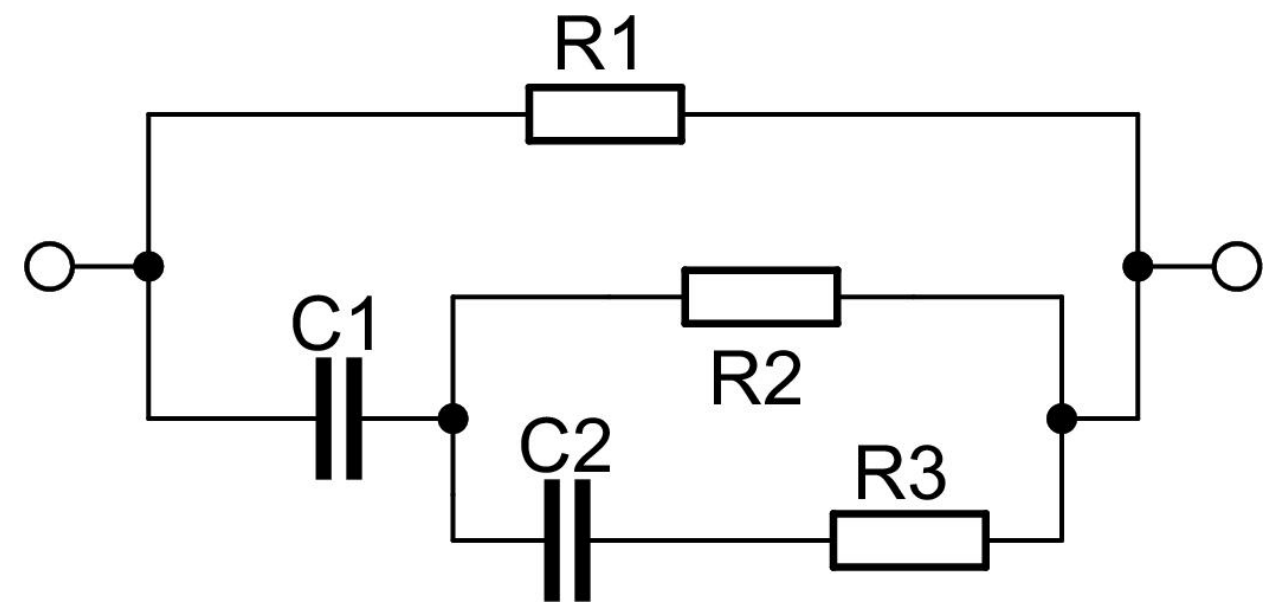

Abbildung 2.7: Ersatzschaltbild des double shell models nach Zhang und Willison (1991).

In Tabelle 2.2 sind die wichtigsten Veröffentlichungen zu elektrischen Eigenschaften mit kapazitiven bzw. multifrequenten Messtechniken chronologisch aufgelistet. Kapazitive Messmethoden werden hier aufgrund der Ähnlicheit zur Impedanzspektroskopie mit aufgelistet und erläutert, obwohl bei diesen Verfahren Impedanzen monofrequent ermittelt werden.

Bereits Anfang der 1970er Jahre fand Chloupek (1972) signifikante Zusammenhänge zwischen der Größe und der Masse von Wurzeln und der elektrischen Kapazität. Wenig später wurden einige Randbedingungen für diese Kapazitätsmethode getestet. Chloupek (1977) untersuchte z. B. den Einfluss des Wassergehaltes des Substrates auf die Kapazität. Kendall u. a. (1982) knüpfen an diese Untersuchungen an und testete verschiedene Pflanzen in Nährlösung und in lehmigem Substrat unter Feldbedingungen. Es wurden zwar signifikante Zusammenhänge zwischen der Trockenmasse von Wurzeln und der Kapazität bei 1 kHz gefunden, jedoch wird darauf hingewiesen, dass der Einfluss edaphischer Faktoren größer als der des Wurzelwachstums ist.

Spätere Veröffentlichungen zeigen, dass die verschiedenen Autoren teils zu gegenteiligen Ergebnissen kommen. So sind bei einigen Autoren die Zusammenhänge zwischen bestimmten Dimensionen des Wurzelsystems (bspw. Wurzelmasse) und der Kapazität z. T. nicht signifikant (z. B. Matsumoto u.a. (2001)). Dalton (1995) fand eine hohe Signifikanz zwischen der Wurzelmasse von Tomaten und der Kapazität, geht aber darüber hinaus explizit auf die Bedeutung 
des Substrates, insbesondere des Wassergehaltes ein. Auch wird auf den hohen Stellenwert der Elektrodenanordnung im Versuchsdesign eingegangen. Darüber hinaus geht es bereits hier darum, eine Zuordnung von biologischen Strukturen zu physikalisch Bauteilen eines Modells (Ersatzschaltbild) zu treffen. Es wird deutlich darauf hingewiesen, dass es Standards für die Technik bedarf, um eine Vergleichbarkeit zu anderen Ergebnissen herbeizuführen.

Ellis u.a. (2012) zeigen, dass die direkte Verknüpfung der Wurzeldimension mit der Kapazität geringe Zusammenhänge liefert.

Wie erwähnt müssen die genannten Veröffentlichungen, die sich mit der monofrequenten Messtechnik unter Berücksichtigung der Kapazität beschäftigen, von den multifrequenten Messtechniken (Impedanzspektroskopie) abgegrenzt werden. Bei der Impedanzspektroskopie wird nicht die Kapazität bei einer definierten Frequenz betrachtet, sondern die Kapazitäten und ohmschen Widerstände nach Berechnung durch ein Inversionsprogramm (siehe Abschnitt 3.4. So sollen bestimmte Größen von Wurzeln separat von „Störgrößen“ betrachtet werden können.

Die Arbeiten, die sich mit der Modellierung einer Pflanze in Nährlösung beschäftigen, sollen hier gesondert beschrieben werden. Aus Tabelle 2.2 sollen besonders die Veröffentlichungen von Ozier-Lafontaine und Bajazet (2005), Repo u.a. (2005) und Cao u.a. (2011) Erwähnung finden.

In Abbildung 2.8 sind die Ersatzschaltbilder für einen Steckling ohne Wurzeln (Abbildung 2.8a bzw. einen Steckling mit Wurzeln (Abbildung 2.8b) dargestellt. R1 und C1 in beiden Modellen stellen die Kapazität und den ohmschen Widerstand des Sprosses oberhalb der Nährlösung dar. In diesen Modellen werden weiterhin die Längsachse des Stecklings bzw. die Grenzfläche zwischen Steckling und Nährlösung modelliert. Der bewurzelte Steckling (Abbildung 2.8b unterscheidet sich vom unbewurzelten (Abbildung 2.8a durch das Vorhandensein einer weiteren seriellen RC-Kombination. Wurzeln stellen sich nach Cao u.a. (2011) also physikalisch durch Kombinationen aus ohmschem Widerstand und Kondensator dar.

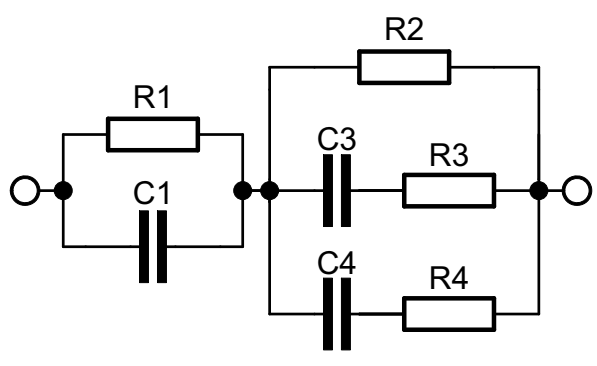

(a)

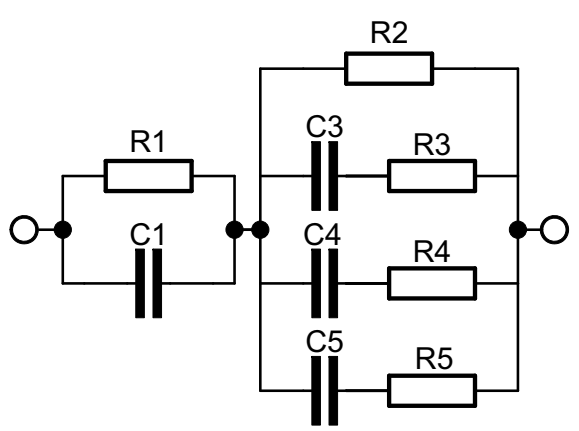

(b)

Abbildung 2.8: Ersatzschaltbilder für (a) unbewurzelte und (b) bewurzelte Stecklinge (Cao u. a. (2011)). Genauere Erläuterung zu den verschiedenen Komponenten finden sich im Text.

Im Gegensatz zu den Ersatzschaltbildern in Abbildung 2.8 ist bei Ozier-Lafontaine und Bajazet (2005) ein anderes Modell zu finden. Nach impedanzspektroskopischen Untersuchungen wurde das in Abbildung 2.9 dargestellte Ersatzschaltbild für die Modellierung der EIS-Messung 
an Tomatenpflanzen gefunden.

Dabei modellieren 4 RC-Glieder die Grenzflächen zwischen Substrat und Wurzeln und Wurzel und Spross. Der vorgeschaltete ohmsche Widerstand (R0) soll das Substrat modellieren.

In diesen Arbeiten geht es um die genaue physikalische Beschreibung eines Modells (Ersatzschaltbild) dieser Konfiguration. Ebenso war man bestrebt, eine Zuordnung der angesprochenen Objektparameter zu den Leitgeweben (Xylem und Phloem) einer Pflanze und den unterschiedlichen Transportwegen in diesem Organ zu finden, so dass das Wachstum bspw. des Wurzelsystems auch mit einer Veränderung physikalischer Größen wie Kapazitäten oder ohmschen Widerständen beschrieben und nachvollzogen werden kann. Besonders in der Arbeit von Cao u. a. (2011) werden die Ersatzschaltbilder genauer erläutert. Diese verändern sich vom unbewurzelten bis hin zum bewurzelten Steckling hinsichtlich ihrer Einzelbausteine.

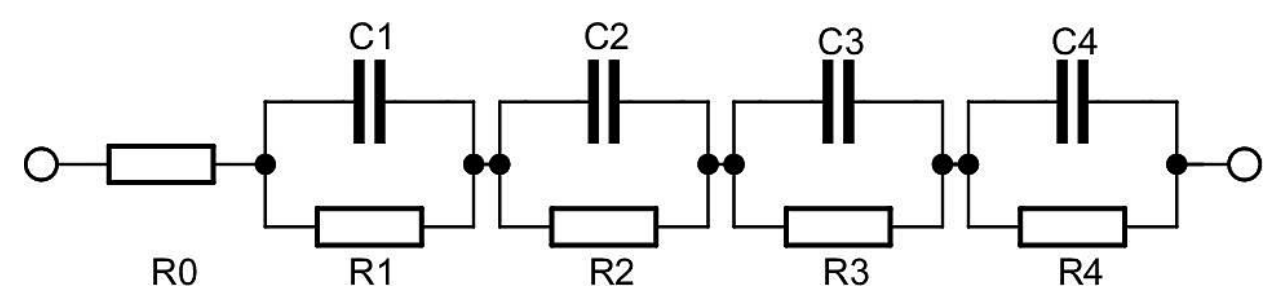

Abbildung 2.9: Ersatzschaltbild aus Ozier-Lafontaine und Bajazet (2005). Genauere Erläuterungen finden sich im Text.

Die hier zusammengetragenen Veröffentlichungen machen deutlich, dass in den meisten Fällen physikalische Messwerte mit bestimmten biologischen Größen korrelieren. Die Ergebnisse, insbesondere die genaue Beschreibung der Pflanze in Form eines Ersatzschaltbildes, scheinen jedoch nicht geklärt. 


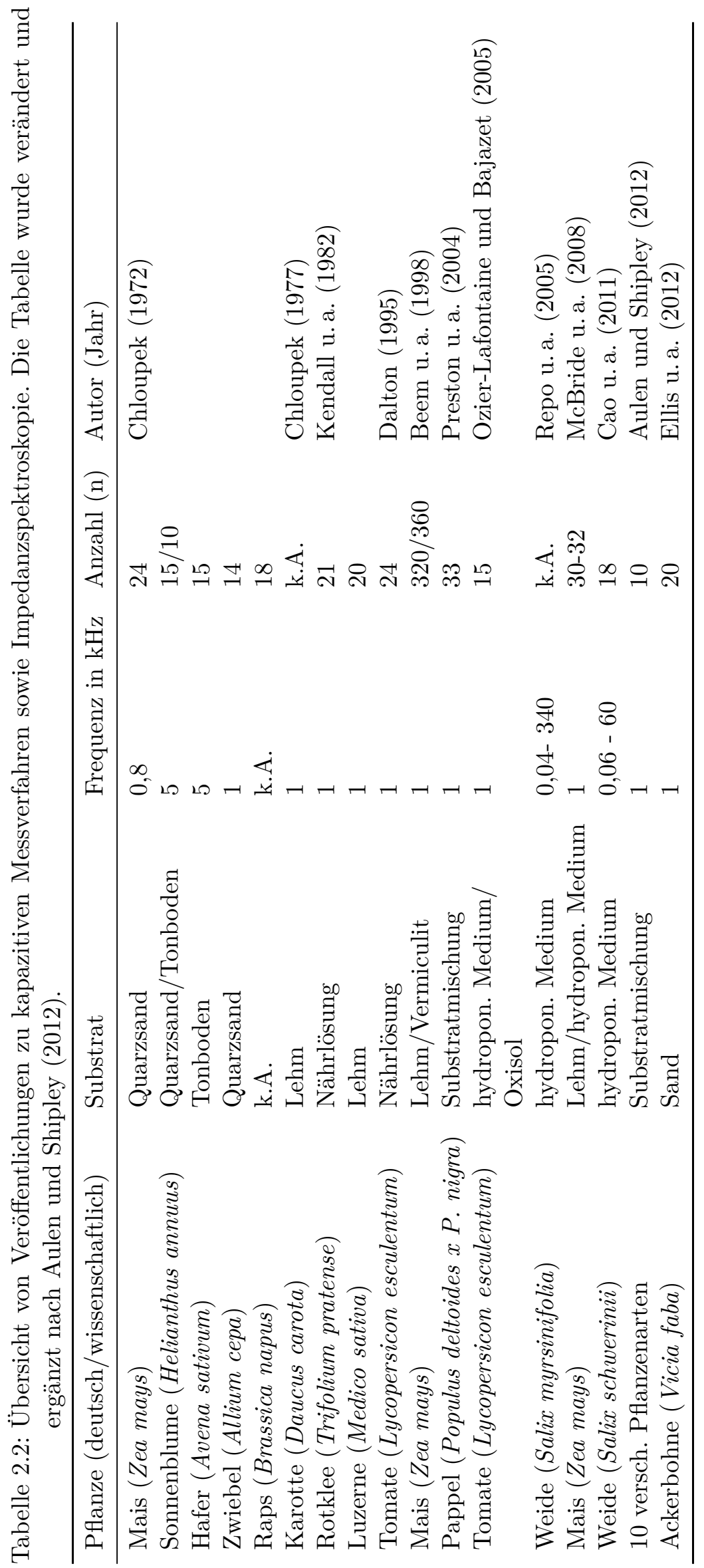





\section{Material und Methoden}

\subsection{Erdungsmessung}

Für die Erdungmessungen im Gelände wurde mit dem GEOHM 5 bzw. mit dem GEOHM 2 der Fa. GOSSEN METRAWATT gearbeitet. Diese Instrumente ermöglichen neben der Bestimmung des Erdungswiderstandes nach allen gängigen Methoden (siehe dazu auch Abschnitt 2.3) auch eine Bestimmung des spezifischen (elektrischen) Widerstandes des Bodens (Erdwiderstand) mit Hilfe der klassischen Vierpunktmessung in Wenner-Anordnung (METRAWATT (2007)). Mit diesen Instrumenten ist es möglich mit zwei verschiedenen Frequenzen zu operieren. Für die hier beschriebenen Messungen wurde die Frequenz von $60 \mathrm{~Hz}$ gewählt.

Abbildung 3.1 zeigt schematisch den Versuchsaufbau der Erdungsmessungen als Drei- und Vierpunktmessung an einem Baum. Bei der Dreipunktmesstechnik (Abbildung 3.1a) wird zwischen dem Baum mit seinem Wurzelsystem (Erder) und der Bodenmatrix ein Stromkreis gebildet. Der Speisekreis führt von der Stromquelle (I) über die Elektroden M/A und B. Die Messung der elektrischen Spannung erfolgt zwischen den Elektroden M/A und N und wird durch den Buchstaben U angedeutet. Bei dieser Art der Messung ist die Elektrode M/A Speise- und Messelektrode zugleich.

Bei der Vierpunktmessung (Abbildung 3.1b) wird der Speisekreis zwischen der Elektrode A und B angelegt. Zwischen den Elektroden $\mathrm{M}_{1(2)}$ und $\mathrm{N}$ wird die elektrische Spannung gemessen. Speise- und Messkreis sind auf diese Weise voneinander entkoppelt. Da bei der Übertragung der Erdungsmessung auf den Baum, wie in Abschnitt 2.2 beschrieben, zahlreiche Übergangswiderstände auftreten können, wird bei den in dieser Arbeit beschriebenen Versuchen auf die Vierpunktmessung zurückgegriffen. Bei der Vierpunktmessung wurden in einem Versuch zwei Erdungswiderstände bestimmt. Der eine wurde mit Hilfe der Elektroden $\mathrm{M}_{1}$ und $\mathrm{N}$, der zweite mit Hilfe der Elektroden $\mathrm{M}_{2}$ und $\mathrm{N}$ bestimmt.

Das Erdungsmessgerät GEOHM 5 ermöglicht sowohl die Einspeisung des elektrischen Stromes, als auch die Messung der Spannung, so dass je nach Messmethode alle drei bzw. vier Elektroden direkt an das Gerät angeschlossen werden. Die Elektrodenbezeichnungen am GEOHM 5 unterscheiden sich zu den hier verwendeten. So wird E für den Erder (A), H für den Hilfserder (B), ES für die Erdungssonde (M) am Baum und S für die Messonde im Boden (N) verwendet. Die Umrechnung der gemessenen elektrischen Spannung in elektrischen Widerstand (Erdungswiderstand) erfolgt nach dem Ohmschen Gesetz (siehe Gleichung (2.1)).

Für sämtliche Erdungsmessungen im Gelände wurden $8 \mathrm{~mm}$ dicke Edelstahlelektroden in T-Form als Hilfserder (B) in ca. $15 \mathrm{~m}$ Entfernung vom Messobjekt ca. $50 \mathrm{~mm}$ in den Boden eingesteckt. Ebenso steckte eine vergleichbare Elektrode in ca. $5 \mathrm{~m}$ Entfernung zum Baum als Messelektrode (N) im Boden. Diese beiden Elektroden wiesen eine Länge von ca. $35 \mathrm{~cm}$ auf. An einer der beiden Schmalseiten der T-Elektrode befindet sich eine $4 \mathrm{~mm}$ Bohrung für die Kon- 


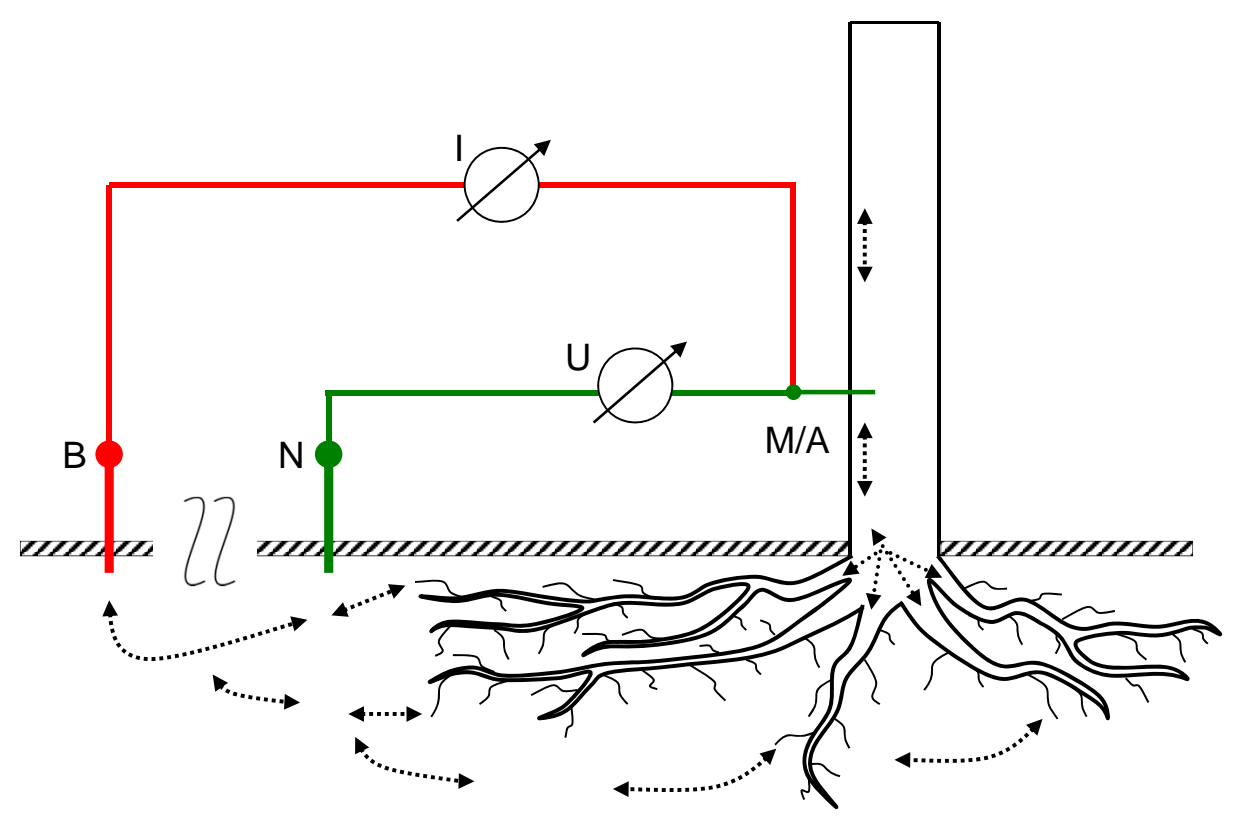

(a)

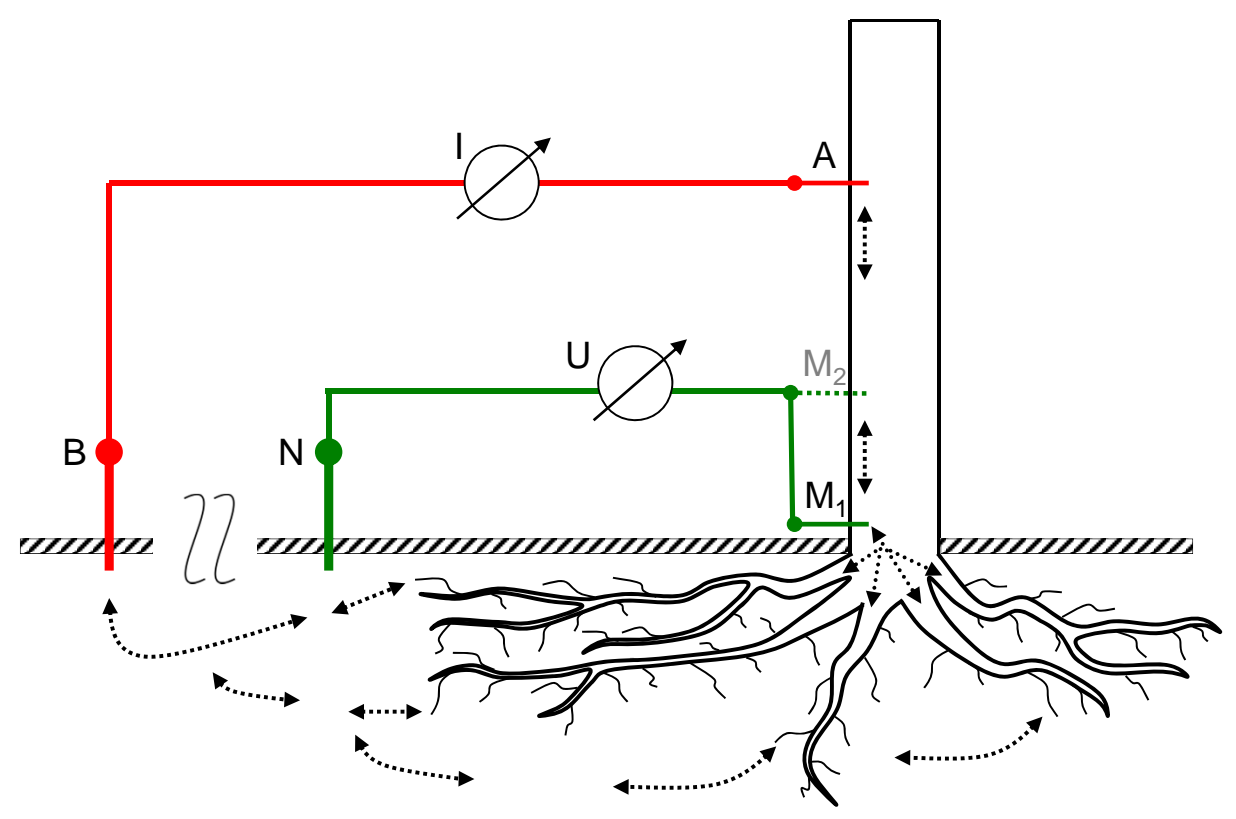

(b)

Abbildung 3.1: Schema der Erdungsmessung am Baum (a) als Dreipunktmessung (klassisch), (b) als Vierpunktmessung. In beiden Einzelgrafiken markieren die roten Linien den Speisekreis mit der Stromquelle (I) und den beiden Speiseelektroden M/A und B bzw. A und B, die grünen Linien markieren den Messkreis mit der gemessenen Spannung (U) zwischen den Messelektroden M/A und N bzw. $\mathrm{M}_{1(2)}$ und N. Bei der Vierpunktmessung (b) wird der Erdungswiderstand unter Berücksichtigung von zwei Messelektroden am Stamm in unterschiedlichen Höhen bestimmt $\left(\mathrm{M}_{1}\right)$ und $\left(\mathrm{M}_{2}\right)$. 
taktierung mit Büschelsteckern. Im Baum wurden jeweils Elektroden verwendet, die aus an der Basis angespitztem Edelstahldraht (2,5 mm dick und $30 \mathrm{~mm}$ lang) bestehen. Zusätzlich wurde dieser Draht mit einer $25 \mathrm{~mm}$ langen und $8 \mathrm{~mm}$ dicken zylindrischen Edelstahlverstärkung als „Kopf“ verschweißt, um das Einschlagen des Drahtes in den Baum bzw. das Herausziehen der Elektrode aus dem Baum nach erfolgter Messung zu erleichtern. Dieser „Kopf“ weist zudem eine $4 \mathrm{~mm}$ Bohrung auf, so dass mit Hilfe von genannten Steckern eine ausreichende gute elektrische Verbindung gegeben ist. Der Edelstahldraht wurde wenige Millimeter in den Baum geschlagen, so dass eine Ankopplung der Elektrode(n) mit den leitenden Geweben des Baumes (Phloem und Xylem) gegeben war. Die Höhe der Stammelektrode A wurde mit $200 \mathrm{~cm}$ festgelegt.

In den hier vorgestellten Tests sollen die Drei- und Vierpunktmessung an Bäumen angewandt und die Ergebnisse miteinander verglichen werden mit dem Ziel, die optimale Messmethode für die Erdungsmessungen an Bäumen zu ergründen. Für die Versuche zur Dreipunktmessung wurden fünf Sommerlinden (Tilia platyphyllos) untersucht. Diese Bäume wurden jeweils wie in Abbildung 3.1a kontaktiert. Nach erfolgter Erdungsmessung mit einer „Baumelektrode“ wurde eine weitere Elektrode auf gleicher Höhe in den Baum geschlagen, diese wurde durch ein ca. $40 \mathrm{~cm}$ langes Kabel mit der ersten Elektrode verbunden. Danach erfolgte eine erneute Erdungsmessung. Dieser Vorgang wurde neunmal wiederholt, so dass die Anzahl der Elektroden sukzessive auf 10 Elektroden erhöht wurde. Der sonstige Versuchsaufbau blieb konstant. Auf diese Weise soll gesprüft werden, ob und inwieweit sich eine Vermehrung der Elektroden am Stamm auf den Erdungswiderstand auswirkt.

Die fünf Sommerlinden wachsen in einer Allee im Kreis Soest (NRW), haben allesamt annähernd gleiches Alter und wachsen unter ähnlichen Bedingungen auf einem schluffig-lehmigen Substrat. Die mittlere Höhe der Bäume liegt bei ca. $15 \mathrm{~m}$. Die Anzahl der Elektroden wurde bei den Dreipunktmessungen nach und nach auf 10 Elektroden vermehrt unter „Kurzschließen“ des Mess- und Speisekreises (auf Höhe der Elektrode M).

Ein vergleichender Test für die Drei- und Vierpunktmessung erfolgte an einem ca. $14 \mathrm{~m}$ hohen Mammutbaum (Metasequoia glyptostroboides) im forstbotanischen Garten in Göttingen. Dafür wurden ebenfalls Dreipunktmessungen mit sukzessiv ansteigender Elektrodenanzahl (bis zu 16 Elektroden) durchgeführt (Abbildung 3.1a). Unmittelbar danach folgte eine Vierpunktmessung, bei der unter Einbeziehung der Elektroden A und M gemessen wurde (Abbildung 3.1b).

Basierend auf den Erkenntnissen der Vorversuche konnten in den Jahren 2009 und 2010 die Hauptversuche an unterschiedlichen Bäumen und Baumarten in Vierpunktmessung durchgeführt werden. Besonders dargestellt wird hier ein Feldversuch (Hauptversuch) an Sommerlinden (Tilia platyphyllos), der in Abschnitt 4.1.3 ausgewertet werden. Hierfür wurden Erdungswiderstandsmessungen in Vierpunktanordnung (Abbildung 3.1b) von 60 Linden unterschiedlichen Durchmessers aus Waldgebieten und Alleen aus dem Göttinger Umland erhoben.

Bei diesem Versuch wurden jeweils zwei Erdungsmessungen pro Baum durchgeführt. Die erste Erdungsmessung erfolgte zwischen den Messelektroden $\mathrm{M}_{1}$ und N. Die Elektrode $\mathrm{M}_{1}$ wurde am Wurzelanlauf auf Bodenhöhe in den Stamm geschlagen. Die zweite Messung wurde mit den Messelektroden $M_{2}$ und $N$ durchgeführt. $M_{2}$ wurde auf einer Höhe von $100 \mathrm{~cm}$ über 
$\mathrm{M}_{1}$ angebracht. Nachdem der erste Erdungswiderstand (im Folgenden E0 genannt) ermittelt war, wurde unmittelbar der zweite Erdungswiderstand unter Berücksichtigung der Elektrode $\mathrm{M}_{2}$ gemessen (im Folgenden E100 genannt). Die Speiseelektrode (A) wurde auf einer Höhe von $200 \mathrm{~cm}$ in den Stamm geschlagen und nicht variiert. Die Stromeinspeisung erfolgte bei diesen Messungen mit nur einer Elektrode (A) am Stamm.

Die Tests sollten zum einen die Frage klären, ob es Zusammenhänge zwischen dem Erdungswiderstand von Bäumen und ihrem BHD gibt, zum anderen, inwiefern die Position (Höhe) der Messelektrode am Stamm ( $\mathrm{M}_{1}$ und $\mathrm{M}_{2}$ in Abbildung 3.1) ausschlagebend für die Erdungsmessung ist. Ferner sollte überprüft werden, ob die Baumart selbst einen entscheidenden Einfluss auf den Erdungswiderstand hat.

In einer weiteren Versuchsreihe folgte die grundlegende Untersuchung, inwieweit Temperatur und Bodenfeuchte die Erdungsmessung eines Baumes beeinflussen. Dazu wurden bei einer Europäischen Lärche (Larix decidua) innerhalb von 22 Std. (9:05 Uhr-07:05 Uhr des darauffolgenden Tages) in unregelmäßigen Abständen drei unterschiedliche Messungen durchgeführt. Neben der Bestimmung des Erdungswiderstandes (E0) in Vierpunktmesstechnik, wurde die Lufttemperatur mit Hilfe eines Thermometers und die Bodenfeuchte mit Hilfe einer Sonde (Moisture Meter type HH2 der Fa. Delta T-Devices) bestimmt, letztere Messung in ca. $5 \mathrm{~m}$ Entfernung zum Stamm. Für diesen Versuch wurde lediglich ein Erdungsmesswert am Baum ermittelt (Messung mit Elektrode M am Stammfuß). Die weiteren Elektrodenkonfigurationen und Abstände wurden die der oben beschriebenen Vierpunktmessung an Linden angeglichen.

\subsection{Bodenwiderstandsmethode}

Die Versuche mit der Bodenwiderstandsmethode gliedern sich in Modellversuche im elektrolytischen Trog und Feldversuche.

In Abbildung 3.2 ist der Versuchsaufbau für die Bodenwiderstandsmethode allgemein als Schema dargestellt. Im Unterschied zu der in Abschnitt 3.1 dargestellten Messanordnung wird hier nicht das gesamte Wurzelwerk durch einen Wert erfasst, sondern es werden viele Einzelmessungen an einem Baum durchgeführt. Dabei wandert die Messelektrode im Boden (N) sukzessive vom Baumstandort in Richtung Speiseelektrode (B). Auf diese Weise wird jeweils zwischen M und N die Spannungsdifferenz gemessen. Bei der praktischen Anwendung werden die gesamten Elektroden für die Bodenmessung vor der eigentlichen Messung in den Erdboden resp. in den Elektrolyten gesteckt. Nach Čermák u.a. (2006) kann die durchwurzelte Fläche eines Baumes geortet werden, da es an den Grenzen dieser Fläche zu einem deutlichen Spannungsabfall kommt, der durch einen scharfen Kontrast anhand der sogenannten „Potentialcharakteristik" sichtbar wird. Die Ansteuerung der einzelnen beschriebenen Elektroden und die Erfassung der Messwerte an den Messstellen erfolgte über eine Multielektrodenapparatur (GeoTom MK-RES/IP/SP) der Firma GEOLOG Fuß/Hepp GbR Augsburg. Über das Programm GeoTom wird diese Multielektrodenapparatur mit Hilfe eines sogenannten Ablaufplans angesteuert. In dem kompakten Messgerät sind sämtliche für die Messung notwendigen Elemente wie Sender, Empfänger, Steuereinheit für die Elektrodenwahl, $\mu$-Controller, 20-Bit- 
AD-Wandler, Stromversorgung und Akkuladeeinheit integriert.

Der Ablaufplan kann derart gestaltet sein, dass einzelne Elektroden nicht geschaltet, während andere sukzessive durchgeschaltet werden.

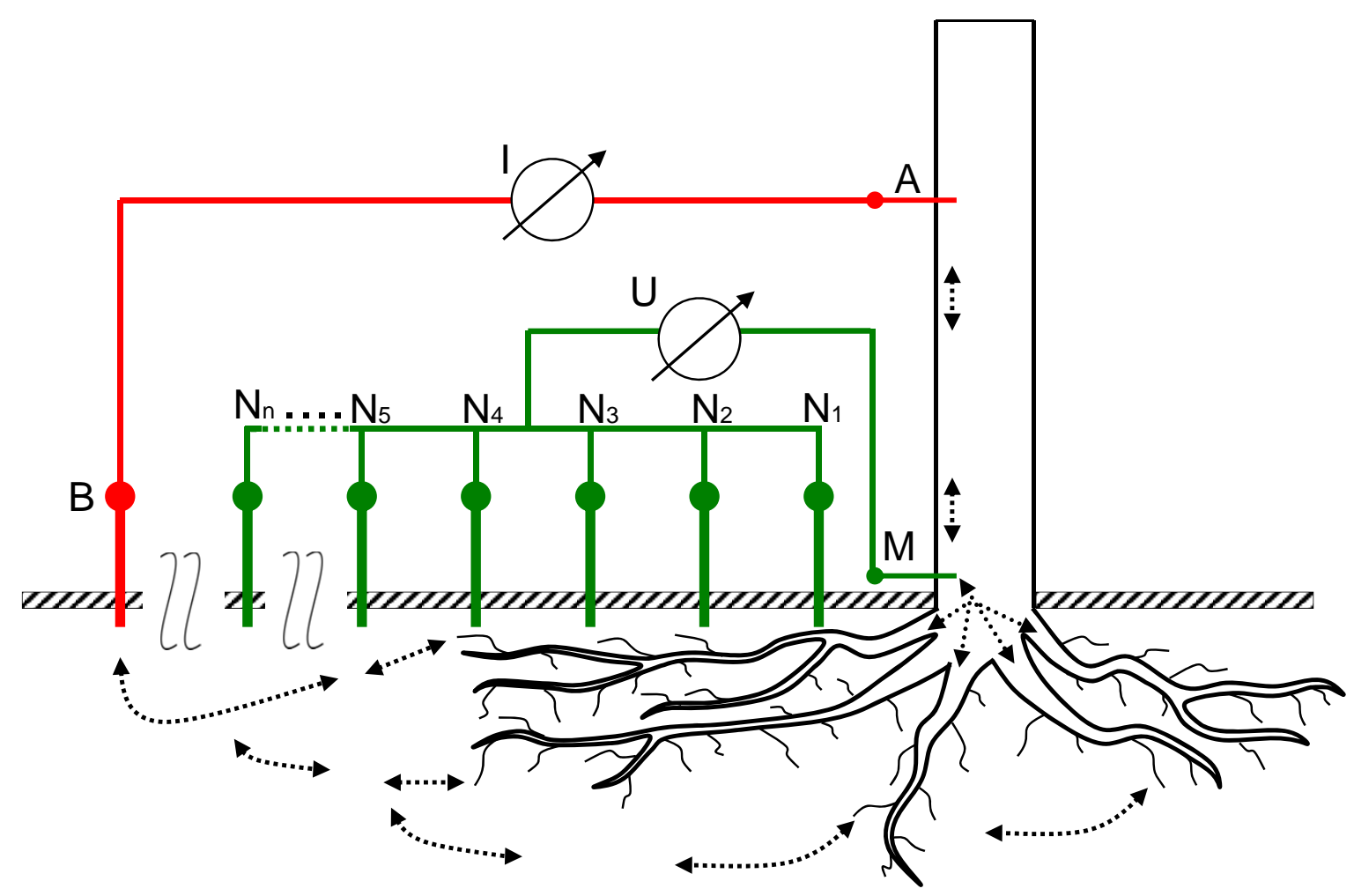

Abbildung 3.2: Schema der Bodenwiderstandsmethode in Anlehnung an Aubrecht u. a. (2006).

\subsubsection{Modellversuche im elektrolytischen Trog}

Der im Folgenden beschriebene Modellversuch sollte klären, ob die von Čermák u. a. (2006) beschriebene Potentialcharakteristik im Modell mit idealen Leitern zu beobachten ist. Zusätzlich wurde die Messung mit Hilfe des Programmes COMSOL Multiphysics® (Multiphysics (2009)) modelliert. Das Programm wird in Abschnitt 3.4 näher erläutert.

Eine geeignete Versuchsanordnung, die zur experimentellen Ermittlung elektrischer Strömungsfelder dient, ist der elektrolytische Trog. Als ein solcher Trog diente bei den folgenden Messungen ein mit elektrolytischer Lösung gefüllter Maurerkübel (Maße: ca. $71 \mathrm{~cm} \mathrm{x} 40 \mathrm{~cm} \mathrm{x}$ $29 \mathrm{~cm}$ ) Die elektrolytische Lösung, mit der der Trog gefüllt war, wurde durch eine Kochsalzlösung $(\mathrm{NaCl})$ verwirklicht $\left(3,7 \mathrm{~S} \mathrm{~cm}^{-1}\right.$ bei $\left.16,3^{\circ} \mathrm{C}\right)$. Die Einstellung dieser ionischen Lösung erfolgte willkürlich. In diesem Milieu wurden Spannungen und Strömungsfelder angelegt, die messtechnisch mit unterschiedlichen Elektrodenkonfigurationen erfasst werden konnten.

Für den ersten Modellversuch wurde als einfaches Baum- bzw. Wurzelmodell ein dreiadriges Kupferkabel gewählt (siehe Abbildung 3.3), bei dem die drei Adern auf einer Seite $1 \mathrm{~cm}$ abisoliert wurden. Die abisolierten Bereiche, die Austrittspunkte des elektrischen Stroms, modellieren die Absorptions- oder Wurzelhaarzone. 


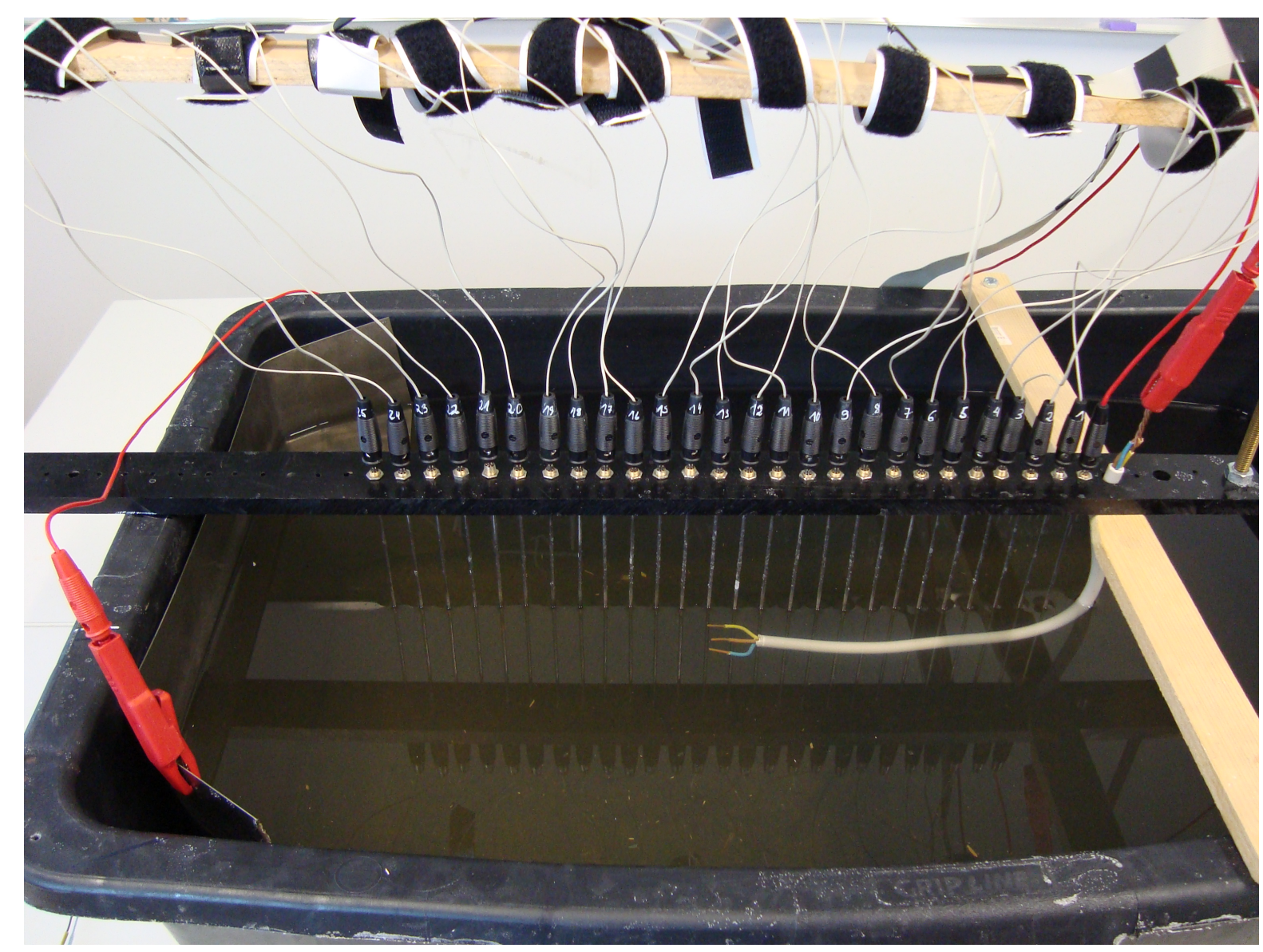

Abbildung 3.3: Foto des elektrolytischen Trogs, einem Maurerkübel, mit den Elektroden, dem Baummodell und der ionischen Nährlösung.

Der restliche, durch einen Kunststoffmantel isolierte Bereich soll den Bereich des Baumes entsprechen, der durch Wurzelrinde oder Borke elektrisch isoliert ist.

Durch die ionische Lösung auf der einen und dem Kupferkabel als sehr guter Leiter auf der anderen Seite, war ein optimaler Stromfluss gegeben. Die Kontaktierung des Wurzelmodells (rechte Seite im Bild) erfolgte über eine Krokodilklemme (Elektrode A). Die drei Adern des Kupferkabels wurden dazu wenige Zentimeter abisoliert, verdreht und die Krokodilklemme aufgesetzt. In die ionische Lösung tauchten 26 Elektroden ca. $2 \mathrm{~mm}$ tief ein. Der laterale Abstand der Elektroden zueinander betrug $15 \mathrm{~mm}$. Die erste Eintauchende (von rechts) ist die Messelektrode $M$, die anderen 25 repräsentieren die zweite Messelektrode $N\left(\mathrm{~N}_{1}-\mathrm{N}_{25}\right)$. Diese 25 Elektroden wurden nacheinander durchgeschaltet, so dass immer zwischen $\mathrm{M}$ und $\mathrm{N}_{1(+\mathrm{n})}$ eine Messung erfolgte. Elektrode N „wanderte“ sukzessive von rechts nach links. Die im Unendlichen gedachte Gegenelektrode (B) war ein in den Trog eingestelltes V2A Edelstahlblech (Maße: ca. $0,1 \mathrm{~cm}$ x $40 \mathrm{~cm}$ x $25 \mathrm{~cm}$ ), ebenfalls durch eine Krokodilklemme kontaktiert (links unten).

Für die zweite Messreihe im elektrolytischen Trog diente bei sonst gleichem Versuchsaufbau eine Kupferscheibe, die das Kupferkabel ersetzte und direkt durch Auflöten eines Kupferkabels als Speiseelektrode (A) diente. In diesem Versuch sollte das Wurzelmodell abstrahiert werden, 
indem die Kupferscheibe(n) als Modell für den gesamten durchwurzelten Bereich eines Baumes diente(n). Es wurde an fünf Kupferscheiben unterschiedlichen Durchmessers $(1 \mathrm{~cm}, 2 \mathrm{~cm}, 4 \mathrm{~cm}$, $6 \mathrm{~cm}$ und $8 \mathrm{~cm}$ Durchmesser) nacheinander gemessen. Der Mittelpunkt der Scheibe lag jeweils unter der siebten Messelektrode $\left(\mathrm{N}_{7}\right), 10 \mathrm{~cm}$ von der Messelektrode $\mathrm{M}$ entfernt in variierender Tiefe.

Im Ablaufplan werden die Elektroden A, M und B fixiert. Wie beschrieben wird lediglich die Elektrode N geschaltet. Die Unterbrechung einer Messung durch Versetzen der Messelektrode ist bei vorher eingerichteter Versuchsanordnung und entsprechendem Messequipment nicht nötig.

\subsubsection{Feldversuch}

Für den Feldversuch wurde die Messanordnung aus Abbildung 3.2 an einem Amberbaum (Liquidambar styraciflua) im forstbotanischen Garten in Göttingen getestet. Für die wandernde Elektrode $(\mathrm{N})$ wurden 25 Elektroden verwendet, die den Erdelektroden aus Abschnitt 3.1 entsprachen. Auch die Elektroden im Baum (A und M) entsprachen denen, die bei der Erdungsmessung verwendet wurden. Es wurde die gleiche Einschlagtiefe der Elektroden für die Ankopplung von Baum und Boden gewählt.

Die Messelektrode (M) wurde unmittelbar am Stammfuss in den Baum geschlagen. Zur Messung der elektrischen Spannung wurden insgesamt vier Messelektroden in jede der vier Himmelsrichtungen geschlagen und miteinander elektrisch verbunden. Hiermit sollte eine homogenere Messung des Stromes erreicht werden. Speiseelektrode A lag $200 \mathrm{~cm}$ oberhalb der gekoppelten Messelektroden M. Hier wurde nur eine Elektrode verwendet. Die Messelektroden im Boden $\left(\mathrm{N}_{1}-\mathrm{N}_{25}\right)$ wurden im Abstand von $0,25 \mathrm{~m}$ in den Erdboden gesteckt. Die erste Messelektrode $\left(\mathrm{N}_{1}\right)$ wurde $0,25 \mathrm{~m}$ vom Wurzelhals des Baumes in den Boden geschlagen. Insgesamt ergab sich daraus eine Auslage der Messlinie von 6,25 m. Es wurden entlang von vier Linien gemessen.

Für die Messungen wurde die bereits in Abschnitt 3.2 genannte Multielektrodenapparatur mit dem entsprechenden Messprogramm (GeoTom) verwendet. Durch die Messung mit 25 Messelektroden im Erdboden konnten 25 Einzelspannungen bzw. Einzelwiderstände ermittelt werden.

\subsection{Impedanzspektroskopie}

Das in dieser Arbeit getestete impedanzspektroskopische Verfahren wurde unter Laborbedingungen an Stecklingen verschiedener Weidenarten durchgeführt. Weiden eignen sich aufgrund ihres schnellen Wachstums und ihrer Fähigkeit, sich vegetativ zu vermehren, besonders gut für die folgenden Grundlagenversuche.

Da sich im Laufe der ersten Messperioden zeigte, dass einige Parameter erheblichen Einfluss auf die Messungen haben, wurden zunächst systematisch Vorversuche zu wichtigen Randbedingungen durchgeführt.

Hauptversuch 1 berücksichtigt die Erkenntnisse aus diesen Vorversuchen. In Hauptversuch 


\section{Material und Methoden}

2 werden zusätzlich wesentliche Aspekte aus dem Versuchsdesign von Cao u.a. (2011) übernommen, um die Versuche miteinander vergleichen zu können.

\subsubsection{Messsystem}

Im Folgenden wird der Versuchsaufbau der Impedanzspektroskopie an einer Laborpflanze dargestellt. Sämtliche Versuche, Vorversuche wie Hauptversuche, liefen in einer begehbaren Klimakammer der Fa. YORK (a Johnsons Controls Company) in einem mehretagigem Lichtregal. Die Kammer hatte eine Größe von ca. $320 \mathrm{~cm}$ x $275 \mathrm{~cm}$ x $220 \mathrm{~cm}$. Eine genau festgelegte Luftfeuchte $(60 \%)$, Temperatur $\left(19^{\circ} \mathrm{C}\right)$ und Beleuchtung konnte in dieser Klimakammer erzeugt und aufrechterhalten werden. Auf dem höhenverstellbarem Lichtregal aus Edelstahl wurde in ca. $130 \mathrm{~cm}$ Höhe die jeweilige Versuchsanordnung auf einem Regalboden aufgebaut. Die Versorgung der Weidenstecklinge mit fotosynthetisch verwertbarem Licht erfolgte über acht Leuchtstoffröhren, die an der Unterseite des darüber hängenden Regalbodens integriert waren. Vier der acht Leuchtstoffröhren erzeugten Warmlicht $(3000 \mathrm{~K})$, während vier weitere für Kaltlicht $(4000 \mathrm{~K})$ sorgten. Die Leuchtstoffröhren waren abwechselnd am Regalboden angeordnet. Auf diese Weise wurden die Pflanzen über ein weites Lichtspektrum versorgt. Die Messung der Temperatur erfolgte neben der jeweiligen Versuchsanordnung auf dem Regalboden.

Das Schaltprinzip in Abbildung 3.4 ist für alle Messungen in gleicher Weise realisiert worden, lediglich die Kontaktierung der Elektroden am Messobjekt unterscheidet sich bei den verschiedenen Messung. Es soll an dieser Stelle stellvertretend für zahlreiche andere Applikationen beschrieben werden. Die genaue Anordnung der Elektroden für die verschiedenen Messungen für die Hauptversuche wird im weiteren Verlauf erläutert.

Das Messgerät, der Impedance Gain Phase Analyzer 1260 (Frequenzganganalysator) der Fa. Solartron/Schlumberger ist mit einem Multiplexer (Siemens Scanner B 8101) verbunden. Die vom Multiplexer ausgehenden Kabel kontaktieren das zu analysierende Objekt (Weidenstecklinge in Küvette oder Becherglas). Zusätzlich ist ein Multimeter (PE Nelson) für die Messungen von Temperatur(en) und Bodenfeuchte in das Messsystem einbezogen. Solartron, Scanner und PE Nelson kommunizieren über ein Bussystem (hpib-Bus)miteinander. Die ermittelten Messwerte, sowohl seitens des Solartrons, als auch seitens des PE Nelson werden über einen GPIB-USB Konverter an einen PC (IBM-Notebook) geleitet. Die Verbindung für die jeweiligen Kanäle (A, B, M, N) sind über kurze koaxiale Leitungen (RG 58) mit BNC-Steckern verwirklicht.

Der Frequenzgenerator mit einem Messbereich von $10^{-6} \mathrm{~Hz}-32 \times 10^{6} \mathrm{~Hz}$ stellt ein wesentliches Subsystem im Frequenzganganalysator (Solartron/Schlumberger) dar. Er erzeugt eine sinusförmige Wechselspannung konstanter Amplitude (0,1 V) zwischen den Polen A (rot) und B (schwarz), also zwischen Steckling und Substrat. Die Messpole sind in Abbildung 3.4 durch die Bezeichnung M (Steckling, gelb) und N (Substrat, blau) verdeutlicht. Am Solartron finden sich hier die Bezeichnungen „hi“ (M) für das hochliegende Potential und „lo“ (N) für das Tiefliegende. Mit Hilfe des Differenzmessverstärkers im Solartron wird zwischen M und N die Spannungsdifferenz gebildet und verstärkt. Das zusätzlich über einen Bus angeschlossene Multimeter (PE Nelson), ermöglicht die Messung von Temperatur und Bodenfeuchte. 


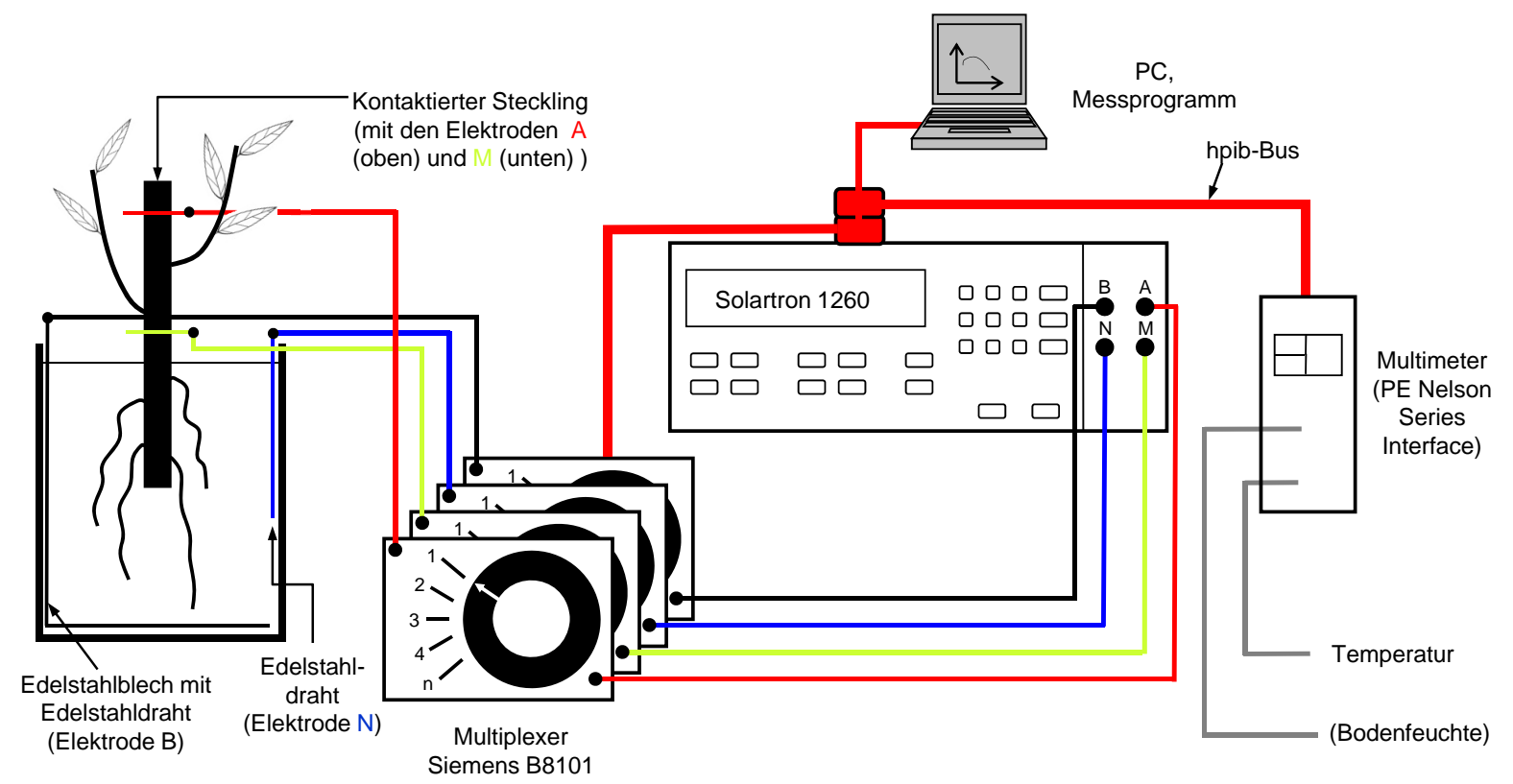

Abbildung 3.4: Aufbau des Messsystems bei der Impedanzspektroskopie (EIS).

Der zwischen Messgerät und Pflanze eingebundene Scanner ist ein mikroprozessorgesteuertes Relaiskoppelfeld mit dem es möglich ist, einzelne analoge oder digitale Signale zu schalten und zu steuern. Ein Multiplexer hat typischerweise einen Ausgang, aber viele Eingänge, so dass die Ausgangsleitung für Informationen mehrfach genutzt wird. Es können Einzelmessungen an beliebigen angeschlossenen Objekten (hier: Weidenstecklinge) durchgeführt werden, oder „durchgeschaltet" werden. Einzelne Relais können entsprechend gesetzt werden, womit ein Signal gewährt wird, während andere Relais nicht geschaltet werden, das Signal ist hier physikalisch unterbrochen. In Abbildung 3.4 ist dies durch die Pfeile am Multiplexer verdeutlicht.

Im Herzstück des Gerätes befinden sich bis zu acht Thermorelaiskassetten, die auf den Verteilerplatten des Gerätes stecken. Eine dieser Kassetten enthält jeweils 20 Relais, die in vier Reihen zu je fünf Relais fest verschaltet sind. Fünf Relaiskontakte bilden dabei mit zwei Arbeitskontakten einen Teilbus. Bei beschriebener Verdrahtung und Ausrüstung ist es möglich bis zu 80 Messobjekte vierpolig nacheinander umzuschalten.

Die Vervielfältigung der Kanaleingänge wurde über eigens hergestellte Steckadapter gelöst, die auf den Relaisanschlusssteckern der Kassetten steckten und verschraubt wurden. Auf die Federleisten dieser Ausgänge wurden Koaxialkabel des Typs RG 174 gelötet, die der entsprechenden Pflanze zugeordnet wurden. Die Kabellänge betrug jeweils $500 \mathrm{~cm}$ bei Hauptversuch 1 und $60 \mathrm{~cm}$ bei Hauptversuch 2. Die Leitungen führten zu den Messgeräten/Systembausteinen außerhalb der Klimakammer. Bei Abzug der Daten erfolgte auf diese Weise keine Störung des Versuches durch Begehung der Klimakammer. Um Einflüsse von außen zu minimieren, wurden geschirmte Zuleitungen gewählt. Die Schirmung der Einzelkabel wurde im Stecker zusammen- 
geführt und auf „ground" gelegt (geerdet). Auf der Seite der Stecklinge wurden die Enden der koaxialen Leitungen auf zwei verschiedene Arten realisiert. Die Kontaktierung zum Steckling (A, M) wurde durch Edelstahlkanülen, die Verbindung zu den Elektroden in der Nährlösung $(B, N)$ durch Aderendhülsen realisiert. Auf der Seite der Stecklingselektroden wurde der kupferne Innenleiter der geschirmten Kabel rückseitig in die hohle Edelstahlkanüle ca. $12 \mathrm{~mm}$ eingeführt und mittels Aderendhülsenzange zusammengedrückt. Bei den Edelstahlelektroden im Substrat wurde die mit dem Innenleiter zusammengedrückte Aderendhülse mit Hilfe einer Lüsterklemme an einen $100 \mathrm{~mm}$ langen und $1 \mathrm{~mm}$ dicken Edelstahlschweißdraht geschraubt, der $60 \mathrm{~mm}$ tief in die Nährlösung tauchte. Im Falle der Elektrode B ist dieser Edelstahldraht mit einem Blech $(0,8 \mathrm{~mm}$ x $20 \mathrm{~mm}$ x $50 \mathrm{~mm})$ verschweißt worden, um die Oberfläche dieser Elektrode zu erhöhen.

Bei den impedanzspektroskopischen Messungen und der Temperaturmessung wurde das auf Delphi basierende Programm „IEEE“ verwendet, das eigens für diese Zwecke programmiert wurde. Mit Hilfe dieses Programmes ist es möglich, den Betrag der Impedanz (|Z|) sowie den Phasenwinkel ф) über der Frequenz sowie die Temperatur zu erfassen und periodisch in angelegte Ordner als ASCII-Tabellen abzulegen. Betrag und Phase bei entsprechender Frequenz mit zugehörigem Datum und Uhrzeit werden dabei in einer Datei abgelegt, die Temperatur mit Datum und Uhrzeit in einer weiteren. Zudem ist im Programm IEEE das Schalten und Setzen der Relais im Multiplexer, sprich die Ansteuerung der verschiedenen Stecklinge, vorgesehen.

Kommerzielle Programme (z. B. Zplot der Fa. Scribner) sind zwar in der Lage über das Bussystem mit dem Frequenzganganalysator zu kommunizieren, allerdings ist die Kommunikation mit anderen Peripheriegeräten, insbesondere mit dem Multiplexer nicht möglich. Für sämtliche Versuche wurde bei sechs Frequenzdekaden und jeweils sechs Frequenzen pro Frequenzdekade gemessen. Als höchste Arbeitsfrequenz wurde $1 \mathrm{MHz}$ gewählt. Auf diese Weise ergaben sich logarithmisch äquidistante Frequenzwerte mit zahlreichen Dezimalstellen. Durch diese sehr dichte Messung über einen weiten Bereich des Spektrums sind besonders gute Interpretationen möglich.

Vor den im folgenden beschriebenen Versuchen wurde das gesamte Messsystem durch Messungen an einer definierten Messanordnung (Testbox) getestet. Da sich das Messsystem als sehr komplex darstellt und mit Hilfe dieses Systems z.T. sehr kleine elektrische Widerstände ermittelt werden sollen, wurde die Genauigkeit der Messungen überprüft. Die Testbox wurde durch eine Leiterplatte realisiert, auf die verschiedene Kondensatoren und ohmsche Widerstände gelötet wurden. Die Testbox mit den jeweiligen Größen der Einzelwiderstände ist in Abbildung 3.5 dargestellt.

Das hier abgebildete Ersatzschaltbild entspricht dem Modell eines bewurzelten Stecklings (vgl. Abbildung 2.8b). Die eingesetzten Elemente (Kondensatoren und ohmsche Widerstände) entsprechen ihrer Größenordnung nach den Ergebnissen der Invertierung der Daten aus der Veröffentlichung von Cao u. a. (2011). In den folgenden Messungen sind somit ähnliche Dimensionen zu erwarten. Auf diese Weise lässt sich das Messsystem unter realistischen Bedingungen überprüfen bzw. testen. 


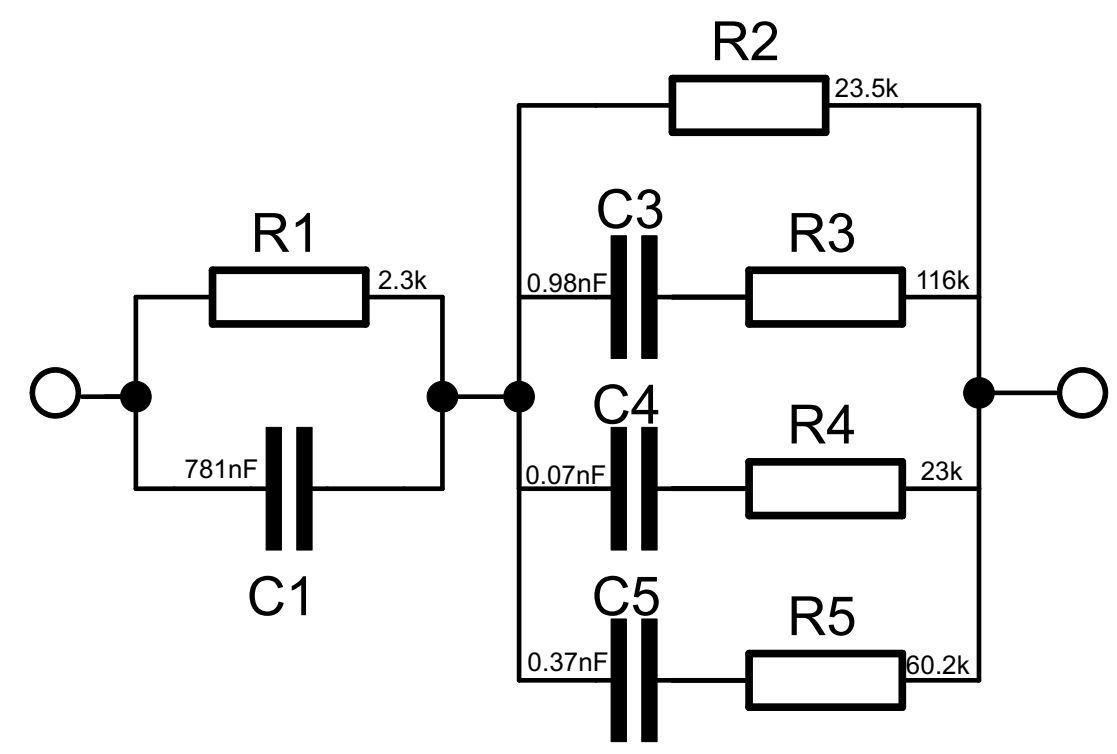

Abbildung 3.5: Darstellung des Ersatzschaltbildes für die Testschaltung mit den verschiedenen Messgrößen.

\subsubsection{Darstellung der Messwerte}

Bei der Darstellung der Messergebnisse aus der Impedanzspektroskopie kommen verschiedene Möglichkeiten in Betracht. Im sogenannten Bode-Diagramm werden einerseits der Betrag der Impedanz und andererseits der Phasenwinkel über der Frequenz aufgetragen. Wobei die Abszisse logarithmisch und die Ordinate linear skaliert werden. Hier kann das Antwortsignal (Impedanz, Phasenwinkel) frequenzspezifisch über einen großen Bereich betrachtet werden.

Die Betrachtung der Ergebnisse in der komplexen Ebene (Complex Plane-plot, Ortskurve oder Nyquist-Diagramm) dient der gleichzeitigen Betrachtung von Imaginär- und Realteil. Diese Darstellungsweise hat den Vorteil, dass Phasenwinkel und Impedanz zugleich betrachtet werden können, außerdem ist eine Vergleichbarkeit mit den Daten einschlägiger Publikationen möglich, die zum großen Teil ihre Ergebnisse in der komplexen Ebene darstellen.

Eine weitere Möglichkeit ist die einer dreidimensionalen Darstellung. Hier werden die beiden Diagrammtypen in der Weise vereint, dass auf der Abszisse logarithmisch die Frequenz, auf der Ordinate der Realteil und auf der Applikate der Imaginärteil Berücksichtigung finden.

Bei der Betrachtung der Messergebnisse wurde zumeist diese Art der Darstellungsweise gewählt, da hier durch entsprechende Veränderung des 3D-Diagramms durch Drehung der größte Betrachtungsspielraum in einem Plot möglich ist und dadurch bessere Interpretationsmöglichkeiten entstehen 

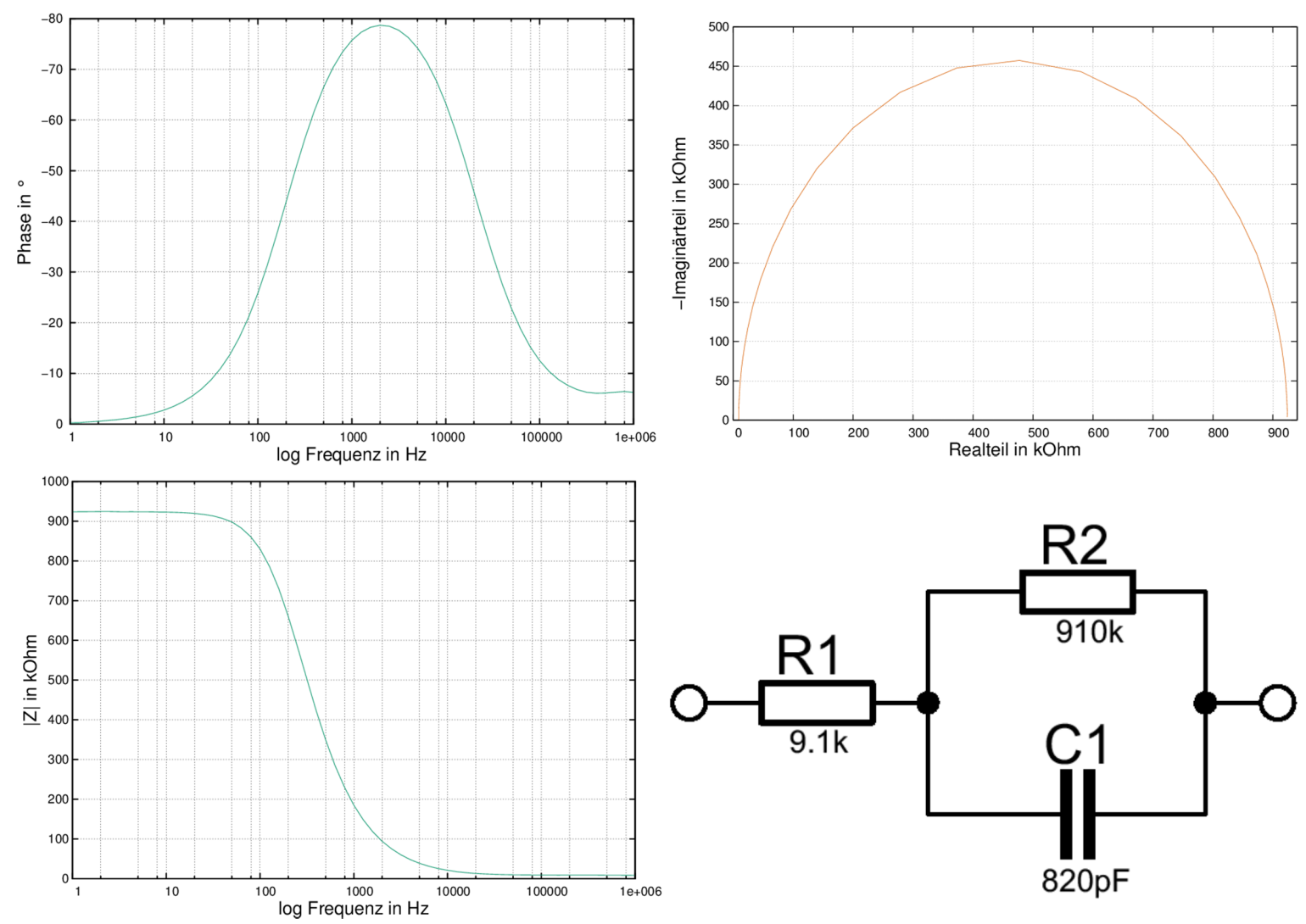

Abbildung 3.6: Verschiedene Darstellungsweisen der gleichen Messung an einer Testschaltung bestehend aus einem R,RC-Glied (vgl. Abschnitt 2.5) (unten rechts), einerseits als Bode-Diagramm (oben und unten links), andererseits in der komplexen Ebene als Nyquist-Diagramm (oben rechts).

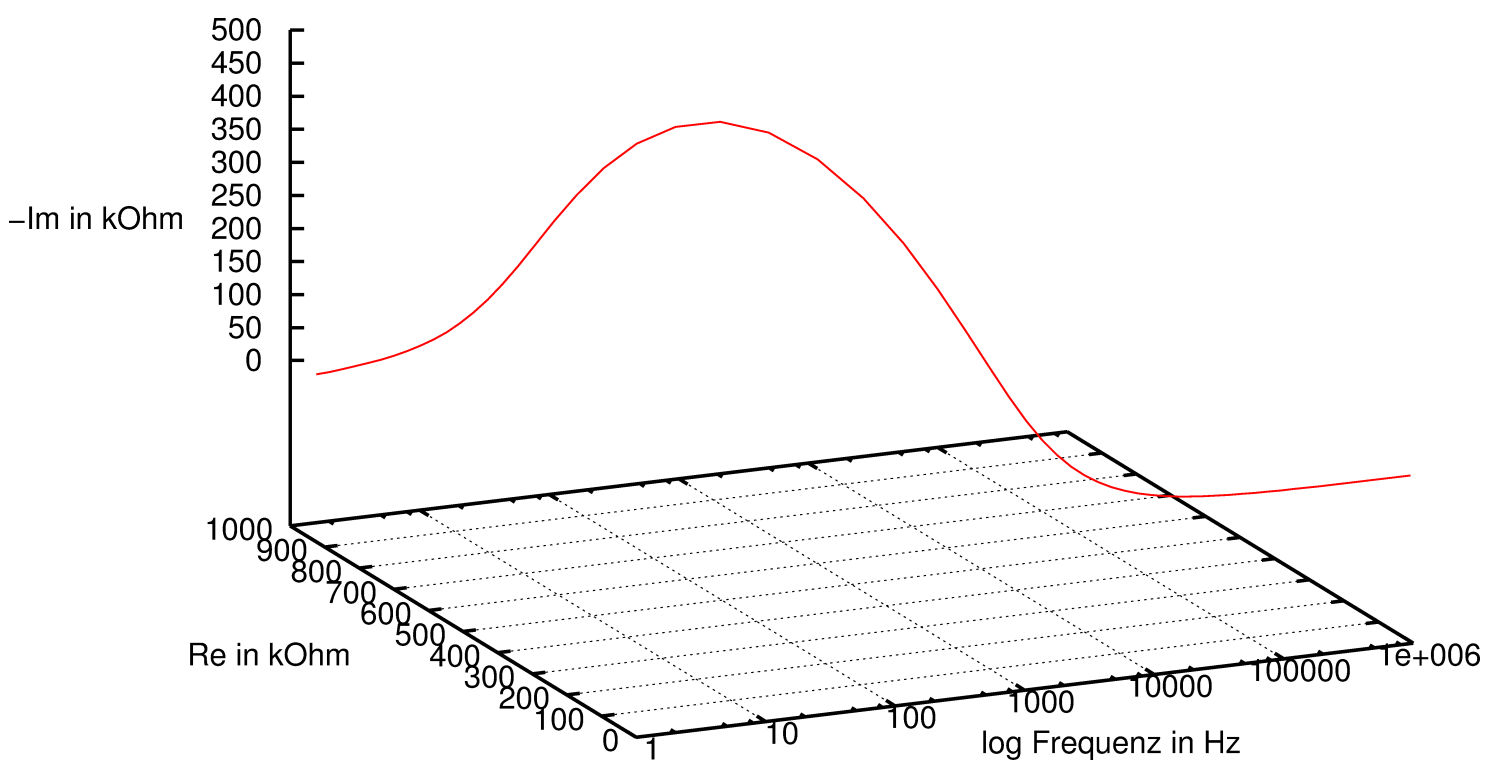

Abbildung 3.7: Darstellung der R,RC-Testschaltung (vgl. Abbildung 3.6 unten rechts) als 3DDiagramm. 


\subsubsection{Differenzbildung von Impedanzspektren}

In dieser Arbeit wurden Spektren von einander abgezogen. Die Differenzbildung von Spektren wird besonders bei zwei Teilversuchen genutzt. Da bei den Untersuchungen des Leitgewebes eines Stecklings der Teil von Realteil resp. Imaginärteil ermittelt werden soll, der einem definierten Stammstück zufällt, wird die Differenz aus zwei Messungen mit unterschiedlicher Messhöhe gebildet.

Auch im Zusammenhang mit Hauptversuch 2 wurden Impedanzspektren von einander abgezogen. Durch die Verkabelung der Stecklinge mit Multiplexer und Messeinrichtung und der Platzierung dieser Systembausteine außerhalb der Messkammer, ergaben sich große Kabellängen. Die Messungen werden zwangsläufig durch Kapazitäten und ohmsche Widerstände dieser Kabel beeinflusst. Daher müssen die zusätzlichen Größen abgezogen werden. Um diesen Anteil zu messen, wurden sweeps an einem dünnen Edelstahlrohr in Nährlösung generiert. Durch die sehr gute Leitfähigkeit dieses Metalls, beeinhalten Real- und Imaginärteil der Impedanz lediglich die verbleibenden Wechselstromwiderstände des System (Systemfunktion). Wird dieser sweep von jedem an einem Steckling gewonnenen sweep abgezogen, so verbleibt das „wahre“ (bereinigte) Spektrum.

Da bei der Impedanzspektroskopie im Gegensatz zu den monofrequenten Messungen mit komplexen Zahlen operiert wird, sollen hier grundsätzliche mathematische Überlegungen angestellt werden. Betrachtet man beispielsweise eine N-gliedrige RC-Anordnung (vgl. Abbildung 2.9), so wird diese mathematisch als

$$
Z_{N}=\sum_{k=1}^{N} \frac{R_{k}}{1+j \omega R_{k} C_{k}} \quad \text { mit } \quad C_{1}=0
$$

dargestellt.

Die Definition des Realteils der Impedanz dazu lautet:

$$
\Re\left(Z_{N}\right)=\sum_{k=1}^{N} \frac{R_{k}}{1+\omega^{2} R_{k}^{2} C_{k}^{2}} \quad \text { mit } \quad C_{1}=0
$$

und die des Imaginärteils:

$$
\Im\left(Z_{N}\right)=\sum_{k=1}^{N} \frac{-\omega R_{k}^{2} C_{k}}{1+\omega^{2} R_{k}^{2} C_{k}^{2}} \quad \text { mit } \quad C_{1}=0
$$

Bildet man nun die Differenz aus einer N-M-gliedrigen Anordnung, so erhält man:

$$
Z_{N}-Z_{M}=\sum_{k=M+1}^{N} \frac{R_{k}}{1+j \omega R_{k} C_{k}}
$$

Daraus erhält man für den Realteil:

$$
\Re\left(Z_{N}-Z_{M}\right)=\Re\left(Z_{N}\right)-\Re\left(Z_{M}\right)
$$




\section{Material und Methoden}

und für den Imaginärteil:

$$
\Im\left(Z_{N}-Z_{M}\right)=\Im\left(Z_{N}\right)-\Im\left(Z_{M}\right)
$$

Diese Überlegungen zeigen, dass in der komplexen Ebene eine Differenzbildung durch einfache Subtraktion von Realteilen bzw. Imaginärteilen geschieht.

\subsubsection{Vorversuche zur Untersuchungen der Randbedingungen}

Die folgenden impedanzspektroskopischen Tests geben einen Einblick in die Empfindlichkeit des Messprinzips und die sensiblen Parameter.

Wenn die Veränderungen der Impedanz über der Frequenz und Zeit diskutiert wird, ist es nötig, sich einen Überblick über die, die Messungen beeinflussenden Faktoren zu verschaffen und ggf. diesen Faktoren entgegenzuwirken oder sie zu eliminieren. Um dies zu leisten, wurden Vorversuche mit einem bestimmten Grundversuchsaufbau durchgeführt. Dieser wird jedoch je nach Faktor in bestimmter Weise angepasst.

Im Rahmen der Vorversuche wurden folgende Faktoren gestestet:

- Einfluss der Elektrodenwahl und der Elektrodenanordnung

- Einfluss der Leitfähigkeit der Nährlösung

- Einfluss des Wasserstandes

- Einfluss der Temperatur

- Einfluss der Dimension der Stecklinge

- Einfluss der Leitgewebe der Stecklinge

In Abbildung 3.8 wird schematisch der Versuchsaufbau für die Vorversuchsreihen gezeigt. Bei den beschriebenen Tests dienten Stecklinge der Echten Trauerweide (Salix babylonica) als Messobjekte. Die Stecklinge bei den folgenden Versuchen waren $20 \mathrm{~cm}$ lang. Der jeweils zu messende Steckling wurde hier durch ein Stativ in seiner Position gehalten. Der Kontakt zwischen Stativklemme und Steckling war durch Styrodur elektrisch isoliert. Der Steckling tauchte in einen zylindrischen Glasbehälter ein, der mit einer Nährlösung der Leitfähigkeit von $245 \mu \mathrm{S} \mathrm{cm}^{-1}$ gefüllt war. Dieser Glasbehälter war auf einer Laborhexe platziert, so dass eine mögliche Veränderung der Eintauchtiefe ausschließlich über die Höhenänderung der Laborhexe geschehen konnte. Zwischen Glasbehälter und Laborhexe befand sich eine Styrodurplatte (schraffierter Bereich), die ebenfalls elektrisch isolierend wirkte.

Der Speisekreis zwischen A und B ist farblich rot markiert, der Messkreis, der durch die Elektroden $\mathrm{M}_{1} / \mathrm{M}_{2}$ und $\mathrm{N}$ verwirklicht ist, ist grün und grau markiert. Die Elektroden $(\mathrm{A}, \mathrm{M})$ wurden durch Edelstahlkanülen, die mit dem Innenleiter des Koaxialkabels verbunden wurden, verwirklicht (siehe dazu auch Abschnitt 3.3.1). Elektrode B ist ein dünnes Edelstahlblech. Die Messelektrode $\mathrm{N}$ hingegen ist ein dünner Edelstahldraht (Schweißdraht). 


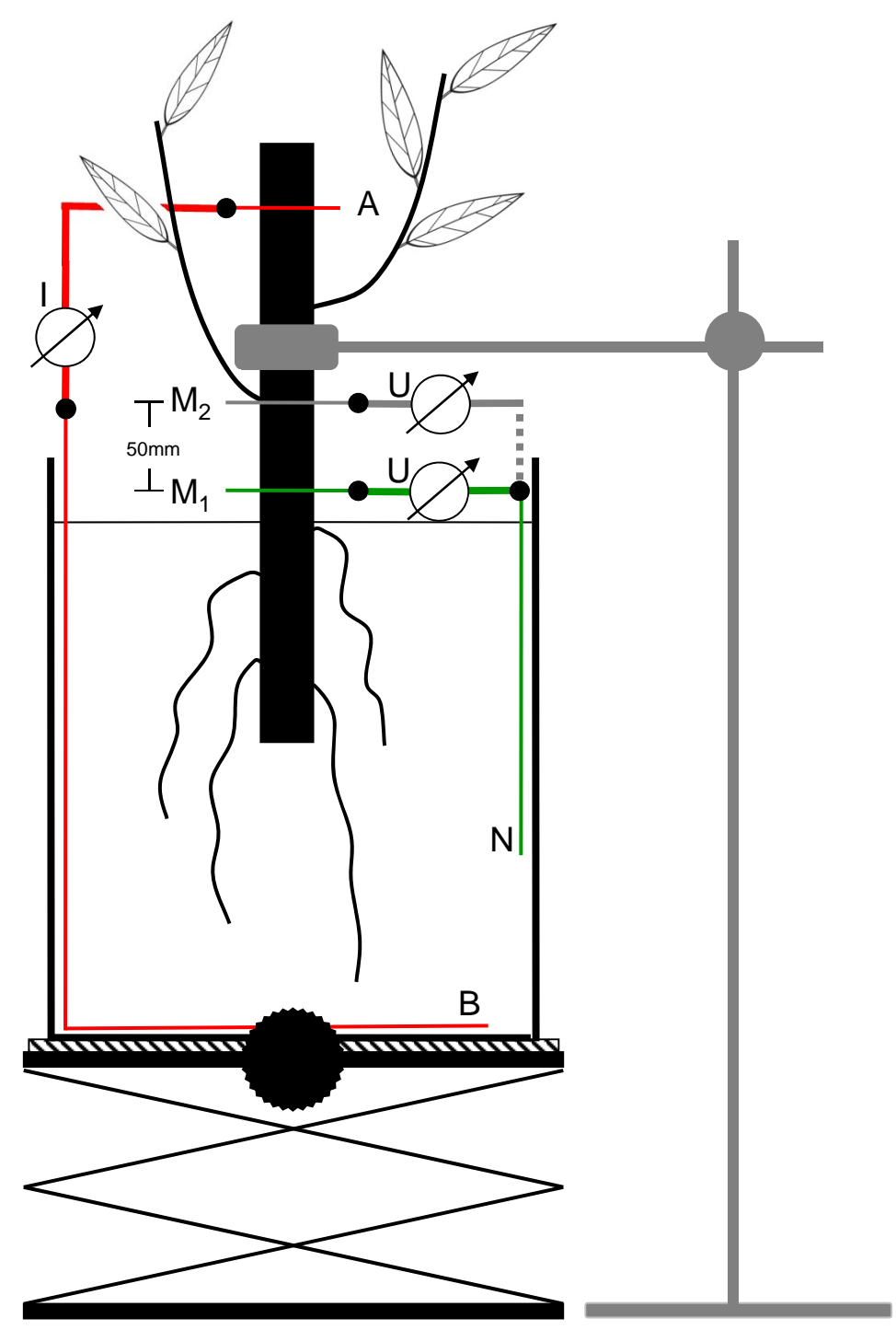

Abbildung 3.8: Schematische Darstellung des Grundversuchsaufbaus zur Untersuchung der Randbedingungen bei der Impedanzspektroskopie an einem Weidensteckling. Weiterführende Erklärungen zu den Linien und Symbolen in dieser Abbildung finden sich im Text.

Für die meisten Messungen sind die Elektroden N und B gekoppelt, so dass in der Regel nur mit Hilfe des Edelstahlbleches gemessen wird. Es wird nicht in jeder Messung mit einem bewurzelten Steckling gearbeitet, da diese Tests Grundlagenversuchen für die Untersuchung der Messkonfiguration entsprechen und daher der Durchwurzelungsgrad sekundär ist. Elektrode A liegt bei den Vierpunktmessungen auf einer Höhe von $18 \mathrm{~cm}$ von der unteren Schnittfläche des Stecklings an gemessen, Elektrode $\mathrm{M}_{1}$ bei $10 \mathrm{~cm}$. Zusätzlich ist eine weitere Messelektrode $\left(\mathrm{M}_{2}\right)$ bei $15 \mathrm{~cm}$ angebracht. Im weiteren Verlauf dieser Arbeit wird die Position der Elektrode $\mathrm{M}_{1}$ als „Messebene 1“ und die Position der Elektrode $\mathrm{M}_{2}$ als „Messebene 2“ synonym verwendet.

Untersucht und diskutiert werden sollen in dieser Arbeit die wichtigsten Einflussfaktoren, die im Folgenden zunächst vorgestellt werden. 


\section{Einfluss der Elektrodenwahl und der Elektrodenanordnung}

In einem Vorversuch wurden zwei vernickelte Stecknadeln (Durchmesser: 0,6 mm) und eine Edelstahlkanüle (V2A) mit einem Durchmesser von $0,7 \mathrm{~mm}$ benutzt. Diese Nadeln wurden allesamt in einen Weidensteckling (Salix babylonica) gesteckt und erst nach 17 Tagen herausgezogen und fotografiert. Vernickelte Stecknadeln wurden zunächst gewählt, um grundsätzlich eine Vorstellung zu erhalten, ob eine Pflanze mit einem Fremdkörper dieser Größe wachsen kann. Weiterhin wurde die Oberfläche einer ähnlichen Edelstahlkanüle (0,8 mm Durchmesser), die ca. 4 Wochen in einem Weidensteckling verblieb, unter dem Rasterelektronenmikroskop hinsichtlich ihrer chemischen Zusammensetzung und möglicher Korrosionsrückstände auf der Oberfläche untersucht. Edelstahl gilt als chemisch besonders verträglicher Stoff, man erhoffte sich auf diese Weise inerte Bedingungen bei der Ankopplung der Elektrode an einem Steckling.

In einem zweiten Test zur Elektrodenwahl- und anordnung wurden impedanzspektroskopische Untersuchungen durchgeführt. In diesem Zusammenhang wurde der Versuchsaufbau aus Abbildung 3.8 dahingehend modifiziert, dass an Stelle der Edelstahlektrode $\mathrm{M}_{1}$ und A auf gleicher Höhe wenige Millimeter lateral versetzt jeweils eine „Silberelektrode“ $(0,8 \mathrm{~mm}$ Durchmesser; versilberter Kupferdraht) durch den Steckling gesteckt wurde. Silberelektroden werden in einigen Veröffentlichungen (z.B. Cao u. a. (2011)) benutzt. Durch Messungen mit diesem Elektrodenmaterial ist ein kritischer Vergleich möglich. Für die Versuche wurden Messungen in Zwei-, Drei- und Vierpunktanordnung mit den genannten Edelstahlelektroden und den hier hinzugekommenen Silberelektroden exemplarisch an einem Weidensteckling durchgeführt. Messelektrode $\mathrm{M}_{2}$ wurde bei diesen Messungen nicht mit einbezogen. Die versilberten Kupferelektroden hatten die gleiche Kontaktfläche mit dem Steckling wie die Edelstahlelektroden. Die Kontaktierung der verschiedenen Elektroden mit dem Messgerät erfolgte über Krokodilklemmen und Koaxialkabel (RG 58).

Tabelle 3.1 gibt einen Überblick über die verschiedenen Messkonfigurationen. Sämtliche Messungen aus dieser Tabelle wurden sowohl mit Silberelektroden, als auch mit Edelstahlelektroden durchgeführt.

Tabelle 3.1: Aufschlüsselung der Messungen für die Untersuchung der Elektrodenannordnung.

\begin{tabular}{ll}
\hline Name der Messung & $\begin{array}{l}\text { einbezogene Elektroden (rot); } \\
\text { gekoppelt }(+) \text {, getrent }(-)\end{array}$ \\
\hline Zweipunktmessung A & $\mathrm{A}+\mathrm{M}-\mathrm{N}+\mathrm{B}$ \\
Vierpunktmessung B & $\mathrm{A}-\mathrm{M}-\mathrm{N}-\mathrm{B}$ \\
Dreipunktmessung C & $\mathrm{A}+\mathrm{M}-\mathrm{N}-\mathrm{B}$ \\
Dreipunktmessung D & $\mathrm{A}-\mathrm{M}-\mathrm{N}+\mathrm{B}$ \\
Dreipunktmessung E & $\mathrm{A}-\mathrm{M}-\mathrm{N}+\mathrm{B}$ \\
\hline
\end{tabular}

Ein Zusammenschluss (Kopplung von Elektroden) erfolgte direkt am Frequenzganganalysator durch BNC-Stecker (Y-Verteiler) und ca. $25 \mathrm{~cm}$ lange Koaxialkabel (RG 174). Die farblich hervorgehobenen Elektroden markieren die in der jeweiligen Messung benutzten Elektroden. 
Ein „+" kennzeichnet die Kopplung, ein „,-“ die Trennung der Elektroden.

Bei der Zweipunktmessungen sind Strom- und Messkreis gekoppelt, so dass am Steckling und in der Lösung immer nur eine Elektrode angeschlossen ist (Zweipunktmessung A). Bei der Vierpunktmessungen B sind diese beiden Kreise von einander entkoppelt. Bei der Dreipunktmessung $\mathrm{C}$ ist das tieferliegende Potential (lo) und die Gegenelektrode zwar jeweils getrennt voneinander, das höherliegende Potential (hi) und die Speiseelektroden am Steckling jedoch gekoppelt. Im Gegensatz dazu sind bei der Dreipunktmessung D die beiden Stecklingselektroden $(\mathrm{A}, \mathrm{M})$ voneinander getrennt, Messung und Speisung in der Lösung erfolgt aber zusammen durch die Elektrode B.

Zusätzlich wurde die Dreipunktmessungen E durchgeführt, diese ist vergleichbar mit der vorherigen Messung (Dreipunktmessung D), jedoch erfolgt Speisung und Messung in Nährlösung bei dieser Dreipunktmessung durch die Elektrode N.

\section{Einfluss der Leitfähigkeit der Nährlösung}

Für die Untersuchung dieses Faktors wurden 15 Stecklinge unterschiedlichen Durchmessers bei drei verschiedenen Nährlösungen gemessen. Die Einstellung der Nährlösung erfolgte durch Herstellung einer Lösung aus destilliertem Wasser und einer entsprechenden Gabe NPK-Düngers (Dünger mit den Nährelementen Stickstoff, Phosphor und Kalium), die Messung und Kontrolle der Leitfähigkeit durch ein Leitfähigkeitsmessgerät der Fa. WTW (Cond 340i).

Neben der bei den anderen Versuchen in diesem Kapitel eingesetzten Nährlösung mit einer Leitfähigkeit von $245 \mathrm{\mu S} \mathrm{cm}^{-1}$ (Lösung 1), wurden Tests in einer Nährlösung mit einer Leitfähigkeit von $54 \mu \mathrm{S} \mathrm{cm}^{-1}$ (Lösung 2) und in einer Nährlösung mit einer Leitfähigkeit von $508 \mu \mathrm{S} \mathrm{cm}^{-1}$ (Lösung 3) durchgeführt. Die drei Nährlösungen wurden ca. 24 Std. vor den Messungen hergestellt und blieben ab diesem Zeitraum in der Klimakammer, so dass die Temperaturen der Lösungen gleich waren. Die Temperaturen der Lösungen wurden unmittelbar vor den Messungen kontrolliert.

Bei diesen Messungen wurden die 15 Stecklinge in Lösung 1, anschließend in Lösung 2 und schließlich in Lösung 3 gemessen. Bei Wechsel der Nährlösung wurden die Stecklinge und der Glasbehälter gründlich ab- und ausgespült.

\section{Einfluss des Wasserstandes}

In dieser Versuchsreihe wurde getestet, welchen Effekt eine Verschiebung des Wasserstands bzw. eine veränderte Eintauchtiefe des Stecklings auf die Impedanz und das gesamte Spektrum hat. Untersuchungsobjekte stellten vier Weidenstecklinge unterschiedlichen Durchmessers dar. Die Durchmesser der Stecklinge waren: $A=9,3 \mathrm{~cm}, \mathrm{~B}=6,6 \mathrm{~cm}, \mathrm{C}=6,1 \mathrm{~cm}$ und $\mathrm{D}=3,9 \mathrm{~cm}$. Die Eintauchtiefe wurde mit Hilfe der in Abbildung 3.8 dargestellten Laborhexe eingestellt. Zuvor wurde die Höhenänderung bei dieser Laborhexe beim Hochdrehen schrittweise bestimmt und durch einen Funktionsgraphen ausgeglichen, so dass die jeweilige Umdrehung und die damit verbundene Eintauchtiefe des Stecklings sehr genau erfasst werden konnte.

Insgesamt wurde bei 15 verschiedenen Höhen (im Falle des Stecklings B, 14 Höhen) in 
einem Bereich von $0 \mathrm{~mm}-78 \mathrm{~mm}$ (B bis $74 \mathrm{~mm}$ ) gemessen. Dabei ist die Nullmarke mit dem knappen Berühren der unteren Schnittfläche des Stecklings mit dem Wassermeniskus definiert. Die einzelnen Höhenstufen sind Abbildung $4.17 \mathrm{zu}$ entnehmen. Zu jeder Höhenstufe wurden jeweils 10 impedanzspektroskopische Messungen (sweeps) pro Steckling durchgeführt, um die Reproduzierbarkeit der Ergebnisse zu überprüfen.

\section{Einfluss der Temperatur}

Da es eine Abhängigkeit des elektrischen Widerstandes von der Temperatur gibt, muss diese bei der Beurteilung der Messergebnisse berücksichtigt werden. Es wurde zunächst angenommen, dass die Temperatur über den Verlauf der Messung durch die Steuerung der Klimakammer konstant gehalten wird. Jedoch stellte sich heraus, dass es in der Klimakammer zu einem Aufheizen beim Anschalten und Abkühlen beim Abschalten der Leuchtstoffröhren durch die zwei unterschiedlichen Phasen, die Tag und Nacht simulieren sollten (Licht- und Dunkelphase), kam. So gab es verschiedene Temperaturniveaus, die durch die Klimakammerregelung nicht nivelliert werden konnten.

Um diesen Effekt genauer zu beleuchten, wurde ein Weidensteckling exemplarisch über einen Zeitraum von 10 Tagen an den Frequenzganganalysator angeschlossen. Über die Steuerung der Klimakammer wurde eine Dauerlichtphase über mehrere Tage und anschließend einen Lichtwechsel mit einer Lichtphase von 7:00 Uhr-19:00 Uhr und eine Dunkelphase von 19:00 Uhr-7:00 Uhr geschaltet. Die Temperatur wurde parallel dazu in der Klimakammer über das Multimeter (siehe Abbildung 3.4 in unmittelbarer Nähe zum Steckling gemessen.

\section{Einfluss der Dimension der Stecklinge}

Zur Untersuchungen der Frage inwieweit die Dimension der Stecklinge das Impedanzspektrum beeinflusst, wurden impedanzspektroskopische Messungen an 15 Weidenstecklingen durchgeführt. Pro Steckling wurden 3 sweeps durchgeführt und diese gemittelt. $\mathrm{M}_{1}$ diente am Steckling als Messelektrode. Sonstige Bedingungen (Eintauchtiefe, Elektrodenmaterial, Nährlösung) wurden hier bei allen 15 Stecklingen konstant gehalten.

Die Stecklinge in dieser Testreihe wurden bewusst in unterschiedlichen Durchmessern (6,79 mm - 20,33 mm) gewählt, um diesen Messparameter möglichst breit zu variieren. Die Länge des Stecklings wurde mit $20 \mathrm{~cm}$ konstant gehalten. Der Einfluss der Länge des Stecklings auf das Impedanzspektrum wurde nicht getestet.

Der jeweilige Durchmesser des Stecklings wurde an drei Stellen (an Stelle der Elektrode A, $\mathrm{M}_{1}$ und $\mathrm{M}_{2}$ ) mit einer Schieblehre gemessen und arithmetisch gemittelt.

\section{Einfluss der Leitgewebe der Stecklinge}

Um die Real- und Imaginärteile der Impedanz zu erhalten, die auf ein definiertes Stück Leitgewebes zurückzuführen sind, wurde an den bereits im vorigen Abschnitt beschriebenen Stecklingen jeweils eine weitere Messreihe durchgeführt. Bei Messung 1 wurde zwischen $M_{1}$ und N gemessen, bei Messung 2 zwischen $\mathrm{M}_{2}$ und N. Die Messelektrode $\mathrm{M}_{2}$ steckte $50 \mathrm{~mm}$ oberhalb 
von $\mathrm{M}_{1}$ (siehe Abbildung 3.8). Bildet man nun die Differenz der Realteile der Impedanz resp. der Imaginärteile der Impedanz zwischen Messung 2 und Messung 1, so erhält man genau den Real- und Imaginärteil der Impedanz, der mit dem Leitgewebe des Stecklings zu erklären ist. Die Legitimation der Differenzbildung von Imaginär- und Realteil der Impedanz wurde in Abschnitt 3.3.3 erläutert.

\subsubsection{Hauptversuche}

Die Hauptversuche gliedern sich in zwei Versuche mit Weidenstecklingen. Zunächst wird ein Versuch dargestellt, in dem die Erkenntnisse aus den Untersuchungen der Randbedingungen mit einfließen. Im zweiten Schritt wird der abgewandelte Versuch dargestellt, der wesentliche Aspekte aus dem Versuchsdesign der Veröffentlichung von Cao u. a. (2011) aufgreift.

\section{Versuchsbedingungen}

Tabelle 3.2 dient der Übersicht über das Versuchsdesign der beiden Hauptversuche zur Impedanzspektroskopie.

Tabelle 3.2: Zusammenstellung der Versuchsbedingungen für die beiden Hauptversuche.

\begin{tabular}{l|ll}
\hline & Hauptversuch 1 & Hauptversuch 2 \\
\hline Messobjekte & Weidenstecklinge & Weidenstecklinge \\
deutscher Name & Echte Trauerweide & nicht bekannt \\
wissenschaftlicher Name & Salix babylonica & Salix schwerinii \\
Anzahl (n) & 15 & 7 \\
Mittendurchmesser & $7 \mathrm{~mm}-10 \mathrm{~mm}$ & $18 \mathrm{~mm}-21 \mathrm{~mm}$ \\
Länge & $20 \mathrm{~cm}$ & $25 \mathrm{~cm}$ \\
Elektrodenmaterial & Edelstahl & Silber \\
Leitfähigkeit (Nährlösung) & $245 \mu \mathrm{Scm}^{-1}$ & $54 \mu \mathrm{S} \mathrm{cm}{ }^{-1}$ \\
Messprinzip & Dreipunktmessung & Zwei- und Dreipunktmessung \\
\hline
\end{tabular}

Für den Hauptversuch 1 dienten 15 Weidenstecklinge (Salix babylonica) aus dem forstbotanischen Garten in Göttingen als Versuchsobjekte. Diese Stecklinge wuchsen über die Dauer des Versuches in einer Plexiglasküvette (Maße: ca. $1,5 \mathrm{~cm}$ x $125 \mathrm{~cm}$ x $25 \mathrm{~cm}$ ), gefüllt mit einer Nährlösung. Die Weiden tauchten $8 \mathrm{~cm}$ in diese Nährlösung ein und wurden durch Holzwäscheklammern, die an eine Stahlleiste fixiert waren, in ihrer Position gehalten (siehe Abbildung 3.9). Die Küvette wurde durch Stege in 15 gleich große Kammern von $8 \mathrm{~cm}$ Breite eingeteilt.

Die Elektrode in der Nährlösung wurde durch ein $5 \mathrm{~mm}$ breites und 0,8 $\mathrm{mm}$ dickes Edelstahlblech verwirklicht. Die Länge des Bleches entsprach der Küvettenbreite. Diese Elektrode wurde an einer Seite mit einem Edelstahldraht verbunden, der seitlich an einer Küvettenschmalseite nach oben geführt wurde und dort durch eine Lüsterklemme mit einem geschirmten Kabel (RG 58) verbunden wurde. Drei Elektroden wurden jeweils komplett durch die Mitte des Stecklings 
gesteckt, so dass die Spitzen auf der gegenüberliegenden Seite deutlich sichtbar waren (siehe Abbildung 3.9p). Eine speisende Elektrode (A) wurde am oberen Ende des Stecklings, $1 \mathrm{~cm}$ unterhalb der oberen Stirnfläche durch den Steckling gestoßen. Des Weiteren wurden zwei Messebenen eingerichtet. Messebene 1 auf der Mitte der Stecklingslänge $(10 \mathrm{~cm})$, Messebene 2 lag bei $15 \mathrm{~cm}$ gemessen von der unteren Stirnfläche des Stecklings. Durch die Messung auf zwei unterschiedlichen Höhen ist es durch Differenzbildung dieser Spektren möglich, eine Vorstellung von der Impedanz eines definierten „Stammstücks“ zu erhalten. Die angesprochene Stahlleiste, die die Stecklinge trägt, diente durch ihre U-Form gleichzeitig als Führung für die Koaxialkabel zu den Stecklingen.

Für den Hauptversuch 2 wurden 7 Weidenstecklinge (Salix schwerinii) von einer Plantage aus Nordfinnland benutzt, die von finnischen Kollegen zur Verfügung gestellt wurden. Die Stecklinge ließ man von Versuchsbeginn bis Versuchsende in einer dafür angefertigten Küvette mit Nährlösung wachsen (Küvettenmaße: ca. $2,5 \mathrm{~cm}$ x $90 \mathrm{~cm}$ x $30 \mathrm{~cm}$ ). Die Weiden tauchten bis zur Hälfte $(12,5 \mathrm{~cm})$ in die Nährlösung ein und wurden durch Styrodurabschnitte in ihrer Eintauchtiefe gehalten. Die Breite der Kammern dieser Küvette betrug $10 \mathrm{~cm}$. Die Elektroden bei diesem Versuch waren Silberelektroden (versilberte Kupferelektroden). Ein 1,0 mm dicker Silberdraht wurde auf Nährlösungsseite am Boden der Küvette gelegt, an einer Seite nach oben herausgeführt und dieses Ende durch Lüsterklemmen mit einem Koaxialkabel verbunden (RG 174), das direkt das Messgerät (Solartron) kontaktierte. Diese Elektrode diente sowohl als speisende, als auch als Messelektrode.
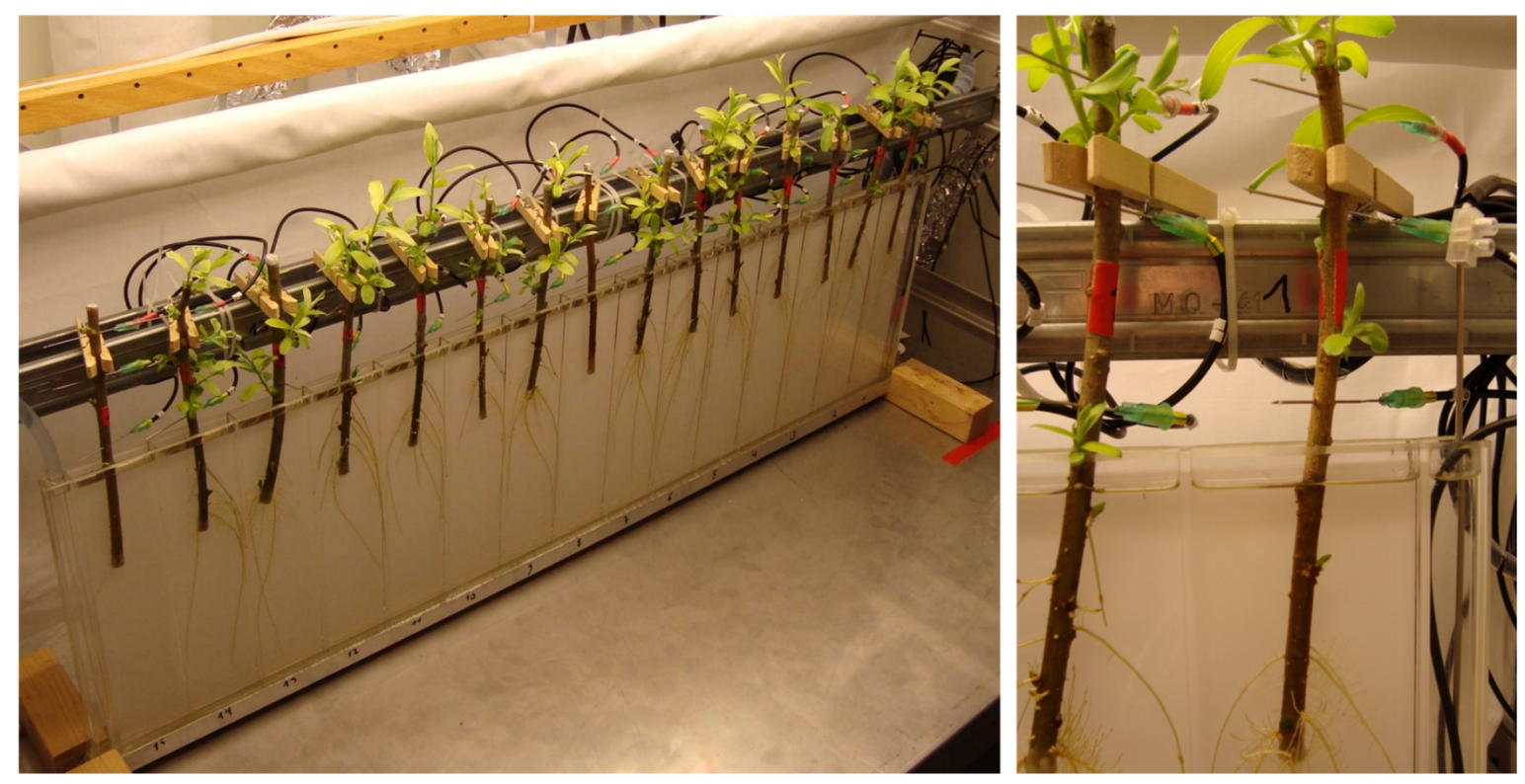

Abbildung 3.9: Fotos des Hauptversuchs 1 in der Klimakammer. Die Stecklinge wachsen in der Klimakammer in einer Plexiglasküvette auf, die durch Stege in gleichgroße Kammern unterteilt wurde. Die Kontaktierung erfolgte, wie beschrieben, durch Edelstahlelektroden.

Auf Stecklingsseite kontaktierte man den Steckling mit der Messeinrichtung an zwei Stellen. Ein $0,8 \mathrm{~mm}$ dicker und ca. $25 \mathrm{~mm}$ langer versilberter Kupferdraht wurde bis zur Hälfte in den 
Steckling auf einer Höhe von $13,5 \mathrm{~cm}$ also $1 \mathrm{~cm}$ oberhalb des Wassermeniskus gesteckt. Eine zweite gleichartige Elektrode kontaktierte $5 \mathrm{~cm}$ oberhalb dieser Elektrode den Steckling auf gleiche Weise. Die Schaltung des Multiplexers wurde so programmiert, dass drei Messungen (sweeps) pro Steckling hintereinander durchgeführt werden konnten. So wurde zunächst eine Dreipunktmessung mit der obersten Elektrode am Steckling als Speiseelektrode (A) und der darunterliegenden Stecklingselektrode als Messelektrode (M) durchgeführt. Die beiden nächsten Messungen an dem jeweiligen Steckling waren Zweipunktmessungen, zum einen unter Berücksichtigung der obersten Stecklingselektrode, zum anderen unter Berücksichtigung der unteren Stecklingselektrode (jeweils A/M). Die Kontaktierung erfolgte mit Hilfe von Lüsterklemmen und geschirmten Leitungen von ca. $60 \mathrm{~cm}$ (RG 174).

In diesem Versuchdesign wurde zusätzlich das bereits in Abschnitt 3.3.3 angesprochene Edelstahlrohr integriert. Dies wurde in ähnlicher Weise wie die beschriebenen Stecklinge kontaktiert. Ein sweep aus Daten vom 02.08.2012 ca. 13:15 Uhr diente als Referenz für die Differenzbildung.

\section{Mariottesche Flasche}

Da bei den Hauptversuchen der impedanzspektroskopischen Messungen mit einer Mariotteschen Flasche gearbeitet wird, sei an dieser Stelle das Verfahren und seine Umsetzung im Versuchsaufbau erklärt.

Bei chemischen Versuchen wird dieses Prinzip genutzt, um bspw. Filter gleichmäßig gefüllt zu halten. Auch zur konstanten Bewässerung landwirtschaftlicher Flächen bedient man sich dieses Verfahrens.

Im hier gewählten Versuchsdesign kommt es auf einen konstanten Stand der Nährlösung in der Küvette über den gesamten Messzeitraum an. Das Verfahren wird dafür entsprechend angepasst (siehe Abbildung 3.10). Für die Untersuchungen wird ein Glasballon von ca. 601 Fassungsvermögen eingesetzt. Dieser enthält die Nährlösung für die Weidenstecklinge und ist durch einen Silikonstopfen luftdicht abgeschlossen. Lediglich zwei Glasröhren von ca. $8 \mathrm{~mm}$ Durchmesser (A, B) werden dabei durch zwei Bohrungen im Stopfen gesteckt. Beide Glasröhren haben Kontakt zur Nährlösung. Ein auf beiden Enden offenes Glasrohr mit Schlauchverlängerung dient zum Überheben der Nährlösung in die Küvette mit den Stecklingen (B). Am Küvettenende des „Überhebers“ ist ein zweites Glasrohr angeschlossen. Ein Weiteres, ebenfalls an beiden Enden offenes Rohr (A), dient zur Einstellung und Aufrechterhaltung des Wasserstandes in der Plexiglasküvette.

Diese Küvette ist durch Stege in Kammern eingeteilt, die an der Basis allesamt untereinander verbunden sind, so dass das Durchströmen der Kammern mit Nährlösung und ein Ausgleich des Wasserstandes in den Kammern gewährleistet ist.

Nach Befüllen der Mariotteschen Flasche, dem Aufstecken des Korkens, dem Einstecken der Glasröhren und dem kurzen Ansaugen der Nährlösung an dem küvettenseitigen Glasrohr B, fließt nun Wasser aus dem Glasballon über das Rohr (B) und die Schlauchverbindung in die Küvette. 
Anschließend dehnt sich die Luft in dem von der Umgebungsluft abgeschotteten Luftreservoir c im oberen Bereich des Glasballons aus, da sich aufgrund der überfließenden Nährlösung vom Glasballon in die Küvette das Volumen des Luftreservois c vergrößert. Der Druck im Reservoir wird folglich geringer (Unterdruck). Wenn der äußere Luftdruck den inneren Druck, der durch die Wassersäule a versursacht wird, und den Druck im Reservoir c übersteigt, bilden sich im unteren Teil des Glasrohres A Luftblasen. Die Schlauchverbindung von B hält die Fließgeschwindigkeit der Nährlösung konstant, da innerhalb und außerhalb des Glasballons der exakt gleiche Luftdruck herrscht. Die Fließgeschwindigkeit wird allein von dem Druck der Wassersäule vom unteren Ende des Glasrohres A und dem unteren Ende des Steigrohres B bestimmt (Wassersäule b1). Die konstante Fließgeschwindigkeit wäre dabei so lange gewährleistet, bis das untere Niveau des Steigrohres A überschritten würde und das Glasrohr nicht mehr in die Nährlösung eintaucht.

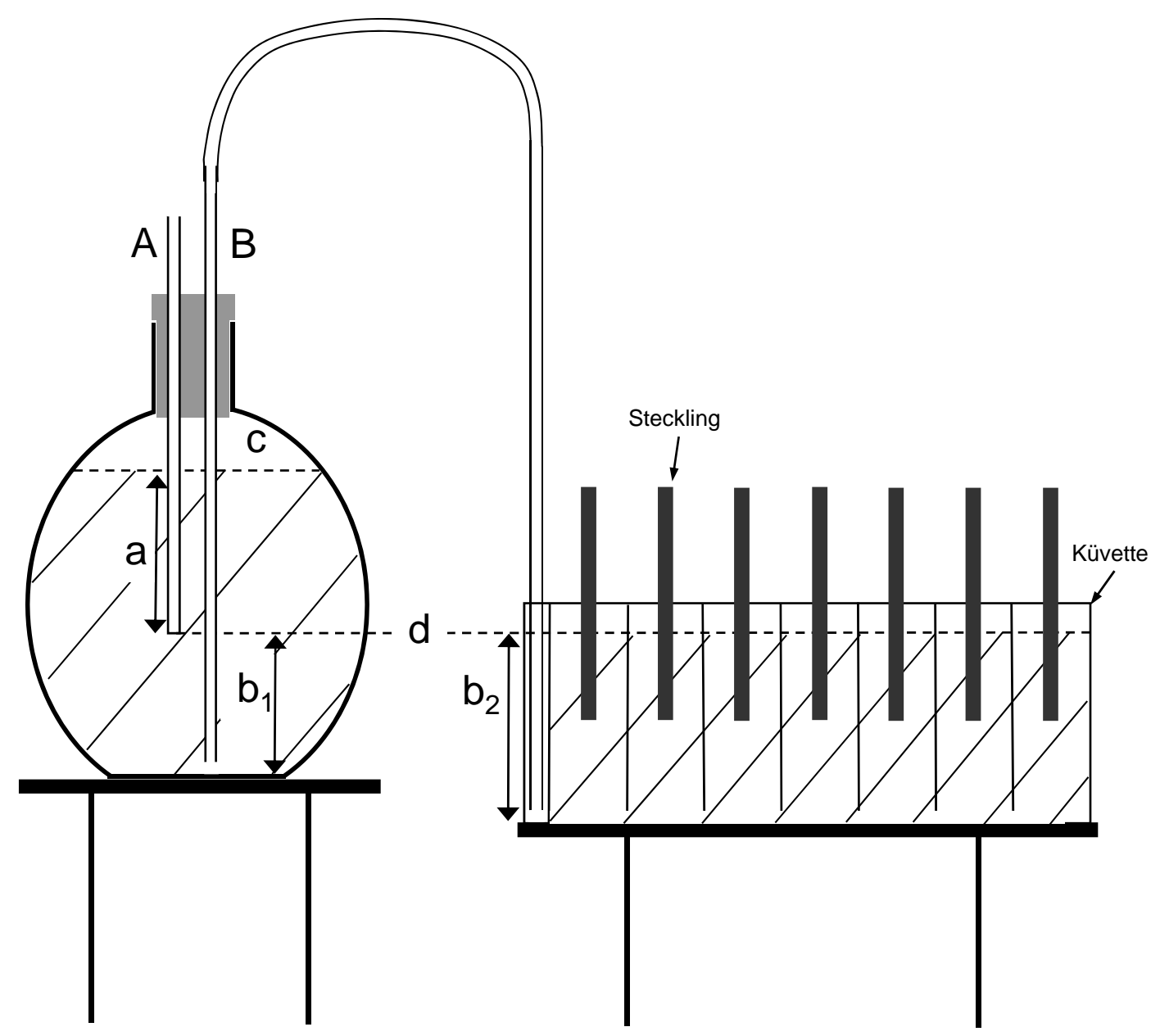

Abbildung 3.10: Schema der Mariotteschen Flasche im Versuchsaufbau.

Im Falle der hier angewendeten Versuchsanordnung, fließt nur so lange Nährlösung durch das Rohr B und seine Schlauchverbindung, bis der Wasserstand der Küvette das Niveau d des unteren Glasrohrendes A im Glasballon erreicht. Bei Verbrauch von Nährlösung in der Küvette durch Transpiration der Blätter der ausgetriebenen Stecklinge oder Verdunstung, sinkt dort die 
Wassersäule b2, folglich reduziert sich hier der Druck. In der Folge fließt so lange Nährlösung in die Küvette nach, bis es zu einem erneuten Druckausgleich kommt und die Flüssigkeit in der Küvette wieder das Niveau d erreicht hat.

\section{Fotografische Auswertung}

Durch die hier angewandte Methodik der Fotografie und Auswertung ist eine zerstörungsfreie Quantifizierung der Wurzeln in situ während des Wachstums möglich. Dieses Verfahren ist von Vorteil, da die impedanzspektroskopische Untersuchung besonders „störungsanfällig“ ist.

Die einzelnen Stecklinge in der Küvette wurden mit Hilfe einer Digitalkamera (Canon EOS 400D und 500D) fotografiert. Es wurden über den Versuchszeitraum nahezu täglich Aufnahmen durchgeführt. Die Kamera stand auf einem Stativ mit ca. 1,2 m Abstand zur Küvette. Aufgrund des lateralen Abstands der Stecklinge zueinander, wurden 3 (-5) Fotos pro Tag gemacht und der Standort des Stativs konstant gehalten. So war gewährleistet, dass bei Versetzen des Stativs der gleiche Abstand eingehalten wurde. In einem Foto konnten drei Küvettenkammern erfasst werden. Die Kameraeinstellung wurde für den gesamten Versuchszeitraum nicht verändert.

Für die ausreichende Beleuchtung wurden auf der Rückseite der Küvette vier $300 \mathrm{~W}$ Halogenstrahler auf Höhe der Oberkante der Nährlösung angebracht. Auf der Rückseite der Küvette wurde zusätzlich eine Milchglasscheibe angebracht. Diese brach das Licht und sorgte für eine gleichmäßige Streuung des Lichts für die Fotografie. Ein weißes Baumwolltuch, das in einem Abstand von ca. $10 \mathrm{~cm}$ auf ganzer Breite der Küvette vor den Halogenstrahlern hing, sorgte wiederum für eine leichte Abdunkelung. Dieses Fotolicht wurde nur für die kurze Dauer der Fotos (täglich ca. 5 Min.) zugeschaltet. So wurde die Störung der sensiblen Messanordnung auf ein Minimum reduziert. Durch die geringe Tiefe der Küvette wuchsen die Wurzeln der Weidenstecklinge primär nach links und rechts in annähernd einer Ebene, so dass durch die Fotos nahezu der gesamte Wurzelbereich erfasst werden konnte. Für jedes Weidenfoto wurde zusätzlich ein Maßstab $(10 \mathrm{~cm} \times 10 \mathrm{~cm}$ kariertes Papier $)$ mitfotografiert. So konnte eine maßstabsgetreue Auswertung durchgeführt werden.

Mit Hilfe des Bildauswertungsprogramms WinRHIZO® (WinRHIZO (2004)) wurden die hochauflösenden Fotos $(2592 \times 3888$ pixel für Hauptversuch 1 und $4752 \times 3168$ pixel für Hauptversuch 2) der Weidenwurzeln genauer analysiert. Die wichtigsten Ergebnisse stellten die Wurzeloberfläche und die Wurzellänge dar. Die Durchmesser und die Durchmesserverteilung der Wurzeln waren für die weiteren Untersuchungen nicht relevant. Die Anzahl der Wurzelspitzen wurde nicht ausgewertet, da teilweise die Wurzeln auf den Fotos mit Hilfe des Programmes fälschlicherweise nicht als ganzes Objekt erfasst wurden, sondern als aneinandergereihte Teilsegmente. Auf diese Weise wurde die Anzahl der Wurzelspitzen überschätzt.

\subsection{Verwendete Programme}

Die Messungen im Zusammenhang mit der Bodenwiderstandsmethode (Modellversuche und Feldversuch) erfolgten mit Hilfe der bereits genannten Multielektrodenapparatur. Das Steuerprogramm GeoTom ist bereits in Abschnitt 3.2 beschrieben worden. 
Die impedanzspektroskopischen Messungen wurden mit dem Programm „IEEE“ durchgeführt. Dieses Programm wurde in Abschnitt 3.3.1 genauer erläutert.

Die Impedanzspektren, die mit Hilfe der bereits beschriebenen Messtechnik entstanden sind, wurden durch verschiedene Programme ausgewertet.

Mit Hilfe des Programms LEVM/LEVMW von James Ross MacDonald ist es möglich, nichtlineare komplexe Kurvenanpassungen für die gemessenen Spektren durchzuführen (MacDonald (1999)). Zuvor müssen geeignete Modelle und die Startwerte der einzelnen Modellparameter für das zu analysierende Spektrum vorgegeben werden. Diese Werte sind zunächst fiktiv. Nach Durchlaufen der Inversion werden dann die echten (berechneten) Zahlenwerte der verschiedenen elektrischen Bauteile (z. B. Kondensator, Widerstand) des zugrundeliegenden Modells angezeigt und in ASCII-Tabellen abgelegt. Bei diesem Programm kann zur Zeit aus 15 verschiedenen Ersatzschaltbildern, die als Grundlage für die Anpassung dienen, ausgewählt werden. In der Regel weicht aber das gesuchte Modell von dem vorgegebenen ab, so dass einzelne Bauteile

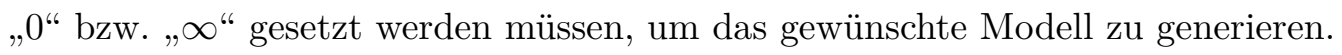

Diese Vorgehensweise ist insbesondere bei sehr komplexen Modellen nicht möglich. In diesen Fällen wurde mit dem Programm ZView gearbeitet (Johnson (2000)). Hier ist es im Gegensatz zu LEVM/LEVMW möglich, Einzelbauteile zu einem Ersatzschaltbild frei zusammenzustellen. Der Handlungsspielraum und die Modellvielfalt ist so deutlich erhöht. Durch die Funktion "Instant fit" können Teile des Spektrums vor dem eigentlichen fit durch festgelegte häufig auftretende vereinfachte Modelle abgeschätzt werden. Diese Funktion erleichtert das Annähern an die wahren Werte des Gesamtmodells. Ein weiteres Programm, mit dem komplexe Kurvenanpassungen möglich sind, ist z. B. das Programm equivalent circuit (Boukamp (1989)).

Allen Programmen ist gleich, dass die errechneten bzw. simulierten Impedanzen, gewonnen aus den definierten, fiktiven Bauelementen des Modellschaltkreises, die kleinstmögliche Abweichung zu den tatsächlich gemessenen Werten (Spektrum) der Probe haben. Die nichtlineare Kurvenanpassung erfolgt dabei mit der Methode der kleinsten Quadrate (Deckwart (2007)).

Mit dem eigens für diese Zwecke entwickelten Programm Impedanz ist es möglich, Impedanzspektren zu generieren. Diese Vorwärtsrechnung entspricht dem gegenteiligen Vorgehen der Inversion. Auf diese Weise ist es möglich, Impedanzelemente eines definierten Modells (Ersatzschaltbild) mit Werten zu belegen und eine fiktive impedanzspektroskopische Messung durchzuführen. Derzeit können Impedanzspektren aus fünf verschiedenen Modellen generiert werden. So ist es möglich, die verschiedenen Werte für die Wechselstromwiderstände in einem Modell zu verändern oder zu variieren und die verschiedenen sweeps miteinander zu vergleichen. Auf diese Weise erhält man eine Vorstellung, wie sich Veränderungen einzelner Komponenten auf das Gesamtspektrum auswirken.

Besonders die Ergebnisse der Impedanzspektroskopie wurden mit Hilfe des Grafikprogrammes Gnuplot 4.4 (Williams, Kelley u. a. (2010)) ausgewertet.

Die Quantifizierung der Wurzeln mit Hilfe der Fotos erfolgte durch das Programm WinRHIZO® (WinRHIZO (2004)). Dieses Programm wird im Abschnitt 3.3.5 („Fotografische Auswertung") genauer vorgestellt.

Für einige modellhafte Betrachtungen und Beobachtungen im elektrolytischen Trog wurde 
das Programm COMSOL Multiphysics® (Multiphysics (2009)) verwendet. Durch die numerischen Simulationen können die tatsächlich durchgeführten Messungen verifiziert und darüber hinaus weiterführende Versuche unter variierenden Bedingungen modelliert werden.

\subsection{Statistische Analysen}

Die statistischen Auswertungen und Darstellungen erfolgten über das Programm R (RCoreTeam (2012)). Dabei wurden einfache lineare bzw. nichtlineare Regressionsanalysen durchgeführt. Es kamen robuste Regressionsmethoden zum Einsatz. Weiterhin wurden die Modelldaten der Impedanzspektroskopie mit Hilfe eines generalisierten, additiven Modells (multivariate Analyse) ausgewertet (Wood (2011), Wang u. a. (2014), Koenker (2015)). 



\section{Ergebnisse}

In diesem Kapitel werden die Ergebnisse zu den drei getesteten Verfahren zur Quantifizierung von Feinwurzeln dargestellt. Quantität und Qualität der Ergebnisse bestimmen die jeweilige Ausführlichkeit der Darstellung. Nach der Veranschaulichung der Ergebnisse der Erdungsmessung folgt die der Bodenwiderstandsmethode. Im Anschluss daran wird die Impedanzspektroskopie aufgrund ihrer Komplexität in besonderer Ausführlichkeit dargestellt.

\subsection{Erdungsmessung}

Nach der Aufbereitung der Ergebnisse zu den Vorversuchen (Einfluss der Elektrodenkonfiguration und anderer Messgrößen) folgt die Darstellung eines ausgewählten Feldversuches (Hauptversuch).

\subsubsection{Einfluss der Elektrodenkonfiguration}

In Abbildung 4.1 sind die Erdungswiderstände fünf verschiedener Sommerlinden (Tilia platyphyllos) bei unterschiedlicher Elektrodenanzahl am Stamm dargestellt. Auf der Abszisse ist die Anzahl der Elektroden, auf der Ordinate der Erdungswiderstand (R) in $\Omega$ aufgetragen.

Die gemessenen Erdungswiderstände liegen insgesamt zwischen maximal $5040 \Omega$ und minimal $1690 \Omega$. Es treten deutliche Unterschiede zwischen dem Erdungswiderstand gemessen mit einer Elektrode am Stamm und $1+\mathrm{n}$ Elektroden am Stamm auf. Betrachtet man den Verlauf der Kurven einer jeweiligen Linde, so fällt auf, dass der Erdungswiderstand mit zunehmender Elektrodenzahl abfällt. Die Beschreibung der Kurve ist dabei nicht linear, sondern asymptotisch. Der Abfall des elektrischen Widerstandes zwischen der ersten und der zweiten Elektrode ist deutlich größer (ca. 24\%-29\%), als bspw. zwischen der neunten und zehnten Elektrode ca. $2 \%-3 \%$.

In Abbildung 4.2 sind die Ergebnisse des Versuches am Mammutbaum (Metasequoia glyptostroboides) dargestellt. Zusätzlich zu den 9 verschiedenen Erdungsmesswerten, ermittelt in Dreipunktmessung, ist der Messwert aus der Vierpunktmessung abgebildet. Letzterer Messwert ist deutlich niederohmiger als sämtliche Werte der Dreipunktmessungen. Außerdem bleibt dieser Wert konstant und reproduzierbar, auch wenn die Elektrodenanzahl vermehrt wird. Gleicht man die Dreipunktmesswerte in einem Graphen mit

$$
f(x)=a+\frac{b}{x^{0.5}}+\frac{c}{x}+\frac{d}{x^{2}}+\frac{e}{x^{3}}
$$

aus und lässt die Variable $\mathrm{x}$ dieser Funktion $(\mathrm{f}(\mathrm{x}))$ gegen $\infty$ laufen, so nähert sich dieser geschätzte Wert (Variable a) dem eigentlich „wahren“ Wert an. Dieser Wert mit „unendlich“ 


\section{Ergebnisse}

vielen parallel geschalteten Übergangswiderständen muss demnach dem Wert der Vierpunktmessung entsprechen. Auf der Abszisse ist die Elektrodenanzahl logarithmisch aufgetragen, auf der Ordinate der Erdungswiderstand. Erkennbar ist der bereits in Abschnitt 4.1 beschriebene, typisch abnehmende Verlauf des elektrischen Widerstandes bei zunehmender Elektrodenanzahl. Die Ausgleichsfunktion ( $\mathrm{f}(\mathrm{x})$ ) nähert sich dem errechneten Erdungswiderstandswert von ca. $888 \Omega$ an. Zusätzlich zu diesem Wert ist der Vierpunktmesswert von $940 \Omega$ parallel zur Abszisse dargestellt. Lediglich eine leichte Abweichung dieses berechneten Wertes zum Vierpunktmesswert von ca. $6 \%$ ist dabei erkennbar. Vergleicht man den Dreipunktmesswert mit nur einer Elektrode an diesem Baum mit dem Vierpunktmesswert, so liegt der Übergangswiderstand bei $3040 \Omega$. Daraus ergibt sich eine Überschätzung des Erdungswiderstandes von ca. $423 \%$.

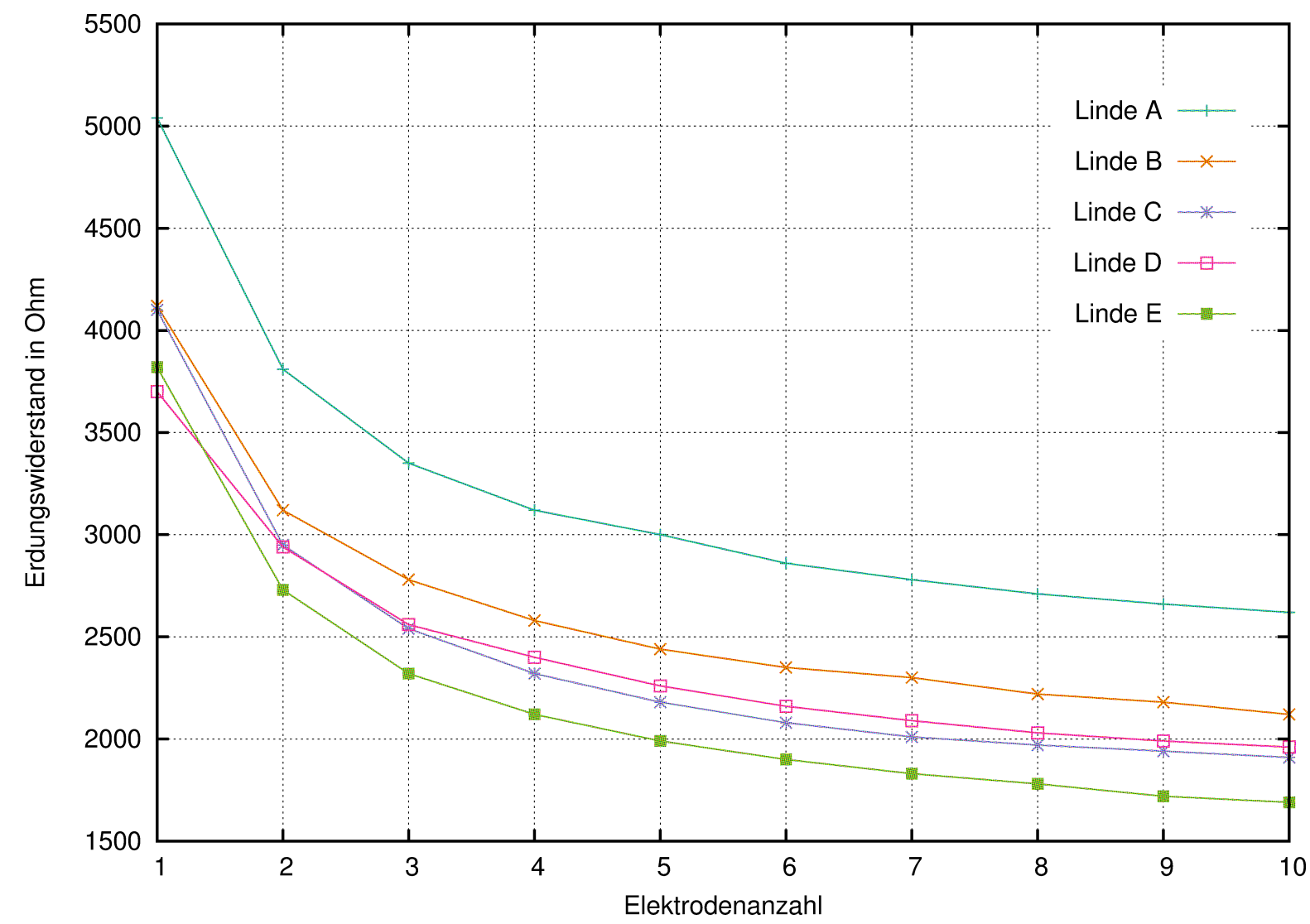

Abbildung 4.1: Erdungswiderstände von 5 Sommerlinden (Tilia platyphyllos) von einer Allee bei unterschiedlicher Elektrodenanzahl. 


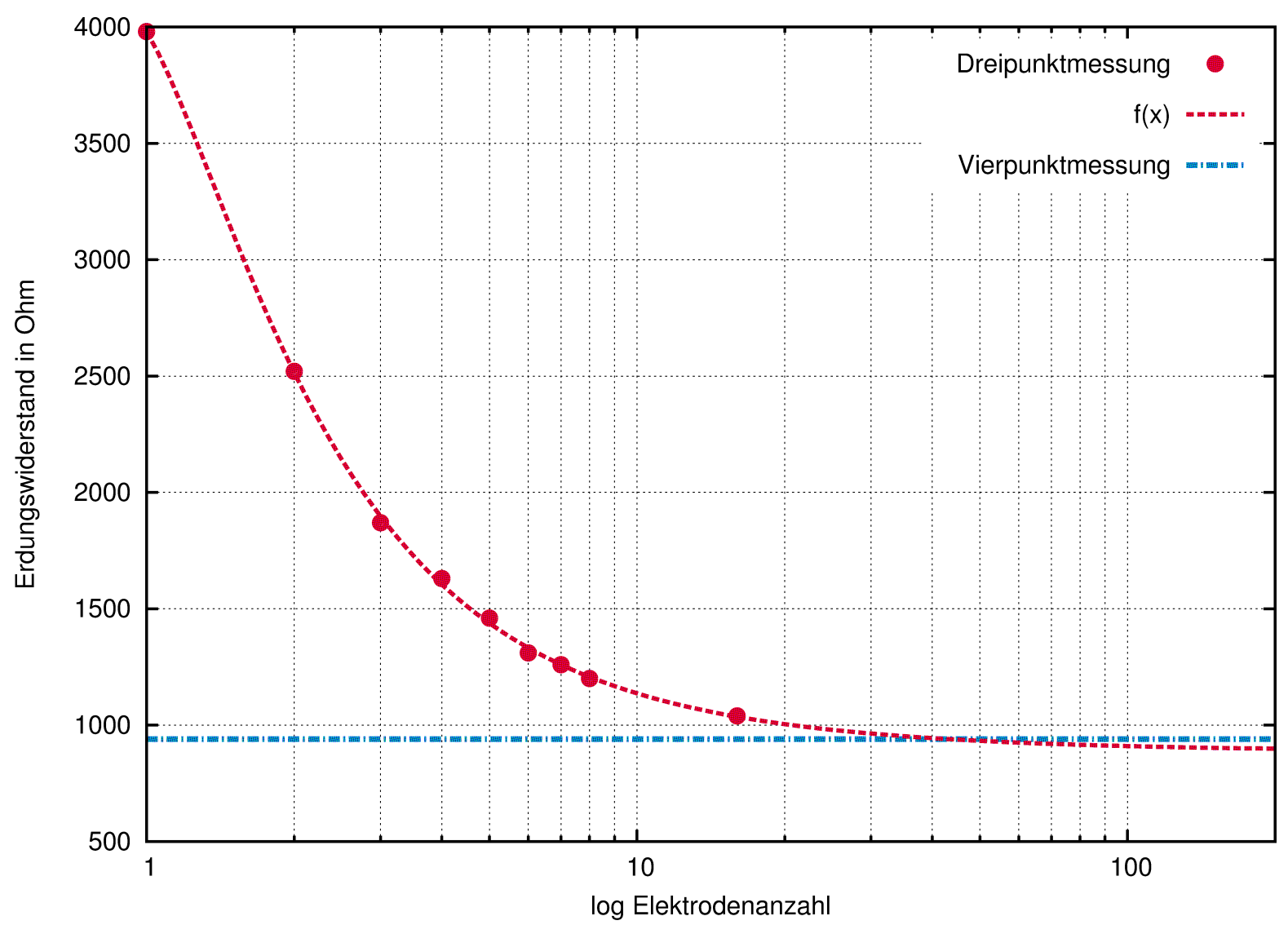

Abbildung 4.2: Erdungsmessungen an einem Mammutbaum mit Ausgleichsfunktion $\mathrm{f}(\mathrm{x})$ und dazugehörigem Vierpunktmesswert.

\subsubsection{Einfluss anderer Messgrößen}

Neben dem Einfluss der Elektrodenkonfiguration wurde, wie beschrieben, der Einfluss der Temperatur und der Bodenfeuchte auf den Erdungswiderstand in einem weiteren Feldversuch an einer Lärche (Larix decidua) untersucht.

Abbildung 4.3 zeigt den Zusammenhang zwischen Temperatur und Erdungswiderstand (links) sowie Bodenfeuchte und Erdungswiderstand (rechts). Die Betrachtungen dieser Zusammenhänge basieren auf zehn/elf Messungen. In beiden Fällen kann ein signifikanter, negativer linearer Zusammenhang zwischen dem Erdungswiderstand und der jeweils betrachteten Messgröße festgestellt werden.

Mit zunehmender Temperatur resp. Bodenfeuchte sinkt der Erdungswiderstand ab. Der Zusammenhang zwischen Erdungswiderstand und Temperatur ist dabei enger als der zwischen Erdungswiderstand und Bodenfeuchte. Bei den Temperaturen traten Unterschiede von $17,7^{\circ} \mathrm{C}$ im betrachteten Zeitraum auf. Die Unterschiede der Bodenfeuchte lagen bei 6,2\%. Die Erdungswiderstände in diesem Zeitraum liegen zwischen minimal $550 \Omega$ und maximal $606 \Omega$. Dies entspricht einem Widerstandsbereich von $56 \Omega$. 

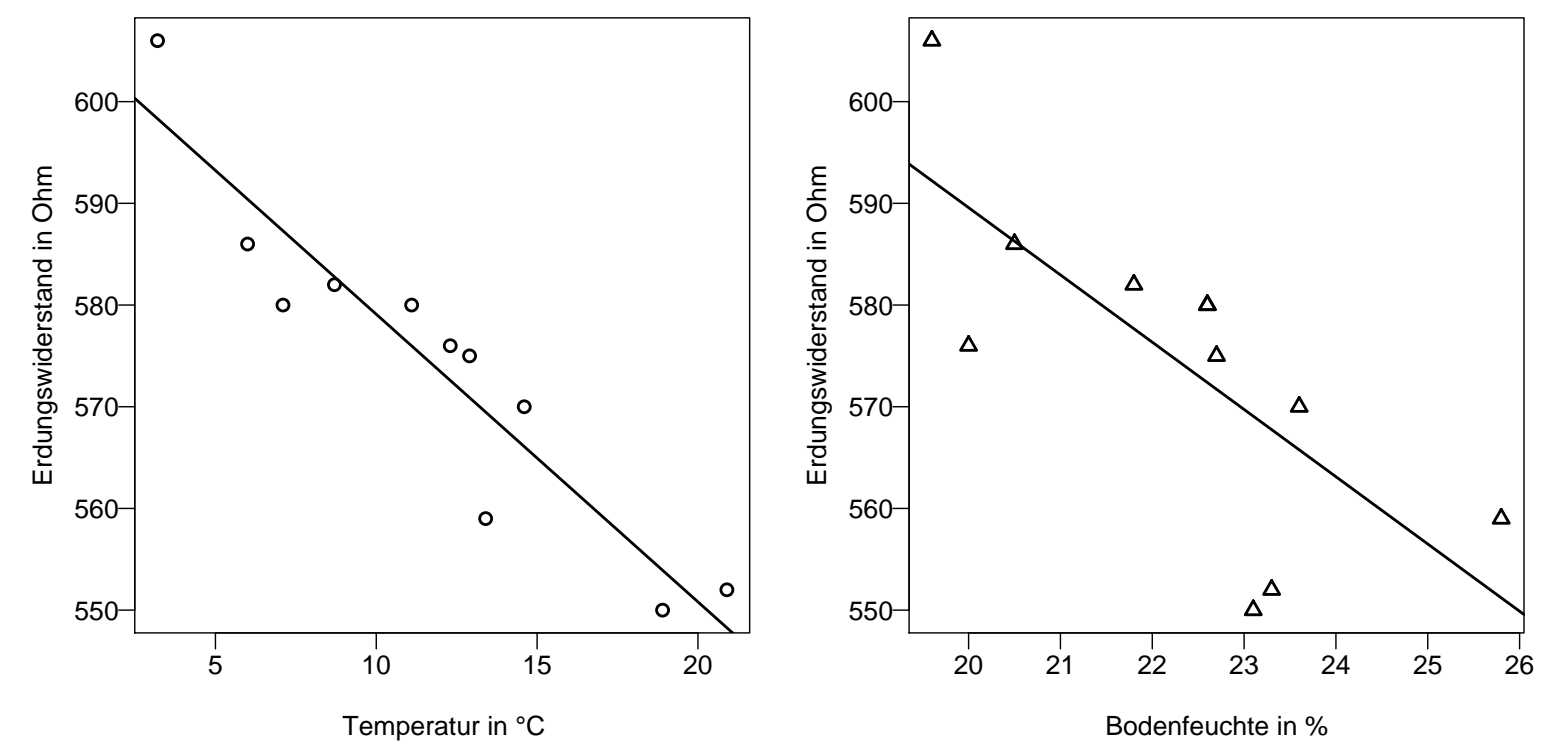

Abbildung 4.3: Zusammenhang zwischen Erdungswiderstand und Temperatur (linke Grafik) und Erdungswiderstand und Bodenfeuchte (rechte Grafik).

\subsubsection{Hauptversuch}

In Abbildung 4.4 werden Zusammenhänge zwischen elektrischen Widerständen (R) und dem Brusthöhendurchmesser (BHD) dargestellt. In der linken Grafik ist der Erdungswiderstand gemessen in Vierpunktanordnung mit der Messelektrode am Stamm $\left(\mathrm{M}_{1}\right)$ im Stammfußbereich dargestellt (E0). In der rechten Grafik ist der Erdungswiderstand, gemessen in Vierpunktanordnung mit der Messelektrode $\left(\mathrm{M}_{2}\right)$ in $100 \mathrm{~cm}$ Höhe abgebildet (E100; schwarz). Die grauen Messwerte sind elektrische Widerstände, die sich aus der Differenz der beiden Messvarianten ergeben.

Die Ergebnisse der Erdungsmessungen in Vierpunktmessung an Sommerlinden (Tilia platyphyllos) zeigen einen deutlichen Zusammenhang zwischen Erdungswiderstand (gemessen in $100 \mathrm{~cm}$ ) am Baum und Durchmesser (BHD). Der Zusammenhang zwischen diesen beiden Größen ist nicht linear, der Graph folgt einer Exponentialfunktion. Je größer der Durchmesser eines Baumes ist, desto geringer ist sein Erdungswiderstand. BHD und Erdungswiderstand korrelieren hochsignifikant. Die Streuung ist gering.

Auffällig ist weiterhin, dass sich der Erdungswiderstand E0 durch deutlich geringere Widerstandswerte von dem Erdungswiderstand E100 unterscheidet. Während die Erdungswiderstände von E0 zwischen minimal ca. $0,046 \mathrm{k} \Omega$ und maximal ca. $1,5 \mathrm{k} \Omega$ liegen, schwanken die Erdungswiderstände zwischen minimal $0,26 \mathrm{k} \Omega$ und maximal ca. $14,27 \mathrm{k} \Omega$. Betrachtet man die Daten, die aus der Differenzbildung der jeweiligen Erdungswiderstandsmessungen hervorgegangen sind, so fällt auf, dass es auch hier einen nicht linearen Zusammenhang mit demselben Gleichungstyp und damit einer ähnlichen Anpassung gibt. Diese Differenz kann so einem „Stammstück“ von $100 \mathrm{~cm}$ zugeschrieben werden. 

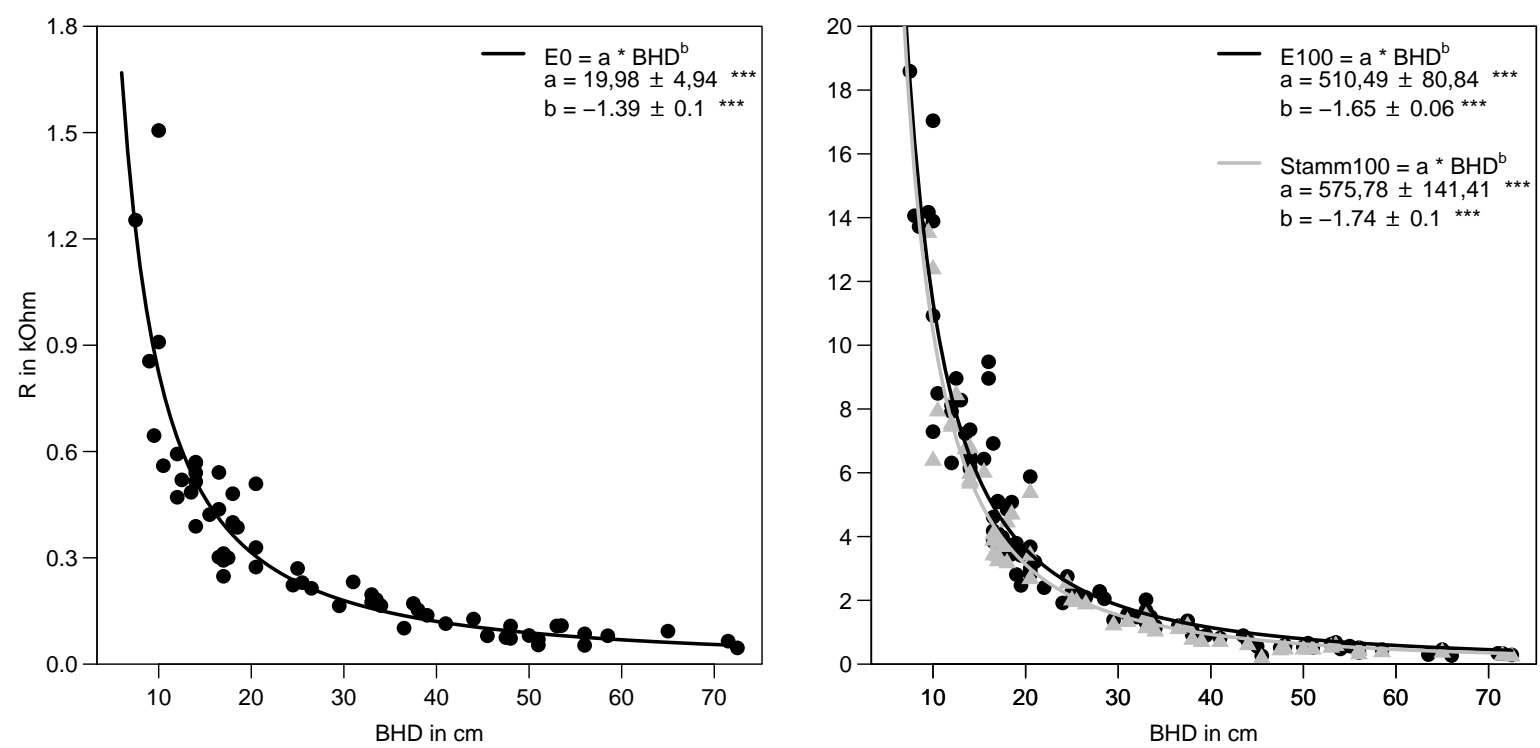

Abbildung 4.4: Zusammenhang zwischen dem Durchmesser eines Baumes (BHD) und seinem Erdungswiderstand, gemessen am Stammfuss (E0, linke Grafik) und in $100 \mathrm{~cm}$ Höhe vom Boden (E100, rechte Grafik). Zusätzlich wird die Differenz der beiden Messvarianten in der rechten Grafik (grau) dargestellt (Stamm100).

Vergleichbare Ergebnisse lieferten Erdungsmessungen an 30 Rotbuchen (Fagus sylvatica) auf weitgehend ungestörten Waldstandorten des niedersächsischen Forstamtes Reinhausen.

\subsection{Bodenwiderstandmethode}

\subsubsection{Modellversuche im elektrolytischen Trog}

In Abbildung 4.5 sind die Ergebnisse des ersten Modellversuches im elektrolytischen Trog aufbereitet. Dargestellt sind die Spannungsverläufe entlang der Messlinie über dem Kupferkabel als Wurzelimitat. Die Spannungskurve hat dort ihren Tiefpunkt, wo die abisolierten Kabelenden liegen (9. Elektrode). Der Abfall der Kurve ist umso stärker, je näher der abisolierte Bereich des Kabels an der Oberfläche liegt. Die Spannungskurve beim Kabelende in größerer Tiefe $(11 \mathrm{~cm}-15 \mathrm{~cm})$ ist sehr flach, hier ist eine exakte Angabe der Koordinaten des Minimums visuell kaum möglich.

Betrachtet man die Ergebnisse der Simulation, so fallen die sehr ähnlichen Kurvenverläufe beider Untersuchungen auf. Die Simulation bildet somit die Messergebnisse sehr gut ab und kann für weitere modellhafte Betrachtungen bei veränderten Messbedingungen eingesetzt werden. 


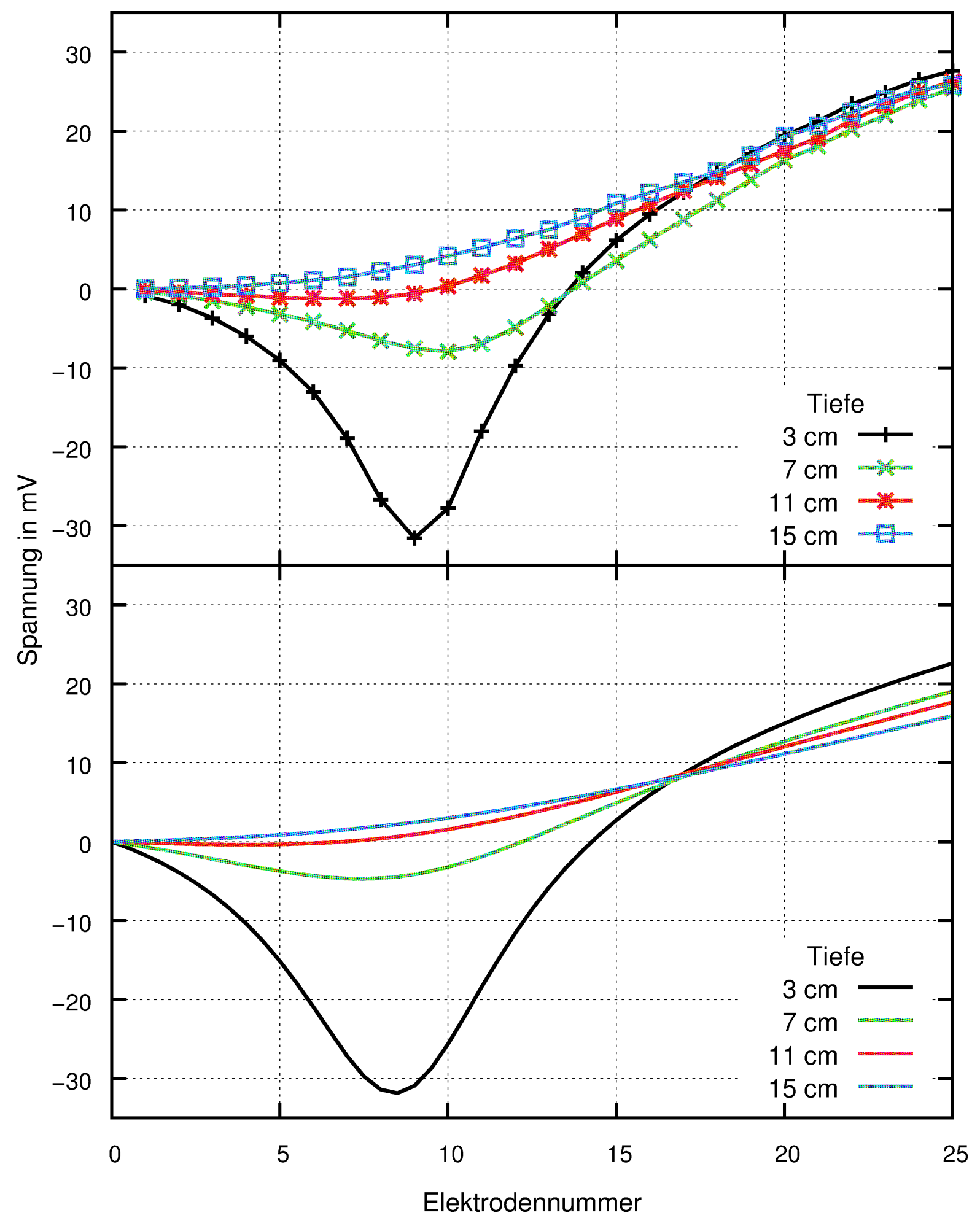

Abbildung 4.5: Ergebnisse der Messung an einem Kupferkabel im elektrolytischen Trog in unterschiedlichen Tiefen (oben) und der dazugehörigen numerischen Simulation (unten).

In Abbildung 4.6 sind die Ergebnisse der Untersuchung von Kupferscheiben als Modelle für den durchwurzelten Bereich eines Baumes dargestellt. Hier sind die Ergebnisse der Messungen an einer Kupferscheibe mit $1 \mathrm{~cm}$ und $8 \mathrm{~cm}$ Durchmesser aufbereitet (Symbole). Bei den hier 
dargestellten Messungen wurden die beiden unterschiedlich großen Kupferscheiben in gleicher Tiefe ( $3 \mathrm{~cm}$ unterhalb der Elektrolytoberfläche) positioniert. Zusätzlich wurden die Ergebnisse einer weiteren numerischen Simulation hinzugenommen (gestrichelte Linien). Zur besseren Vergleichbarkeit wurden die Kurven so normiert, dass der Tiefpunkt beider Kurven bei $0 \mathrm{~V}$ liegt. Der Ursprung der Absizze wurde hier mit der Position der Elektrode M als Bezugselektrode definiert. Vergleicht man zunächst die modellierten Daten mit den Messwerten, so fällt auf, dass sich besonders im Falle der $1 \mathrm{~cm}$ Scheibe die Messwerte und die Daten aus der Simulation stark ähneln. Größere Abweichungen sind lediglich in der Peripherie der Messlinie bei der $8 \mathrm{~cm}$ Scheibe zu erkennen.

Bei der Untersuchung der Wirkung dieser Kupferscheiben konnte ein deutlicher Unterschied im Kurvenverlauf festgestellt werden (siehe Abbildung 4.6).

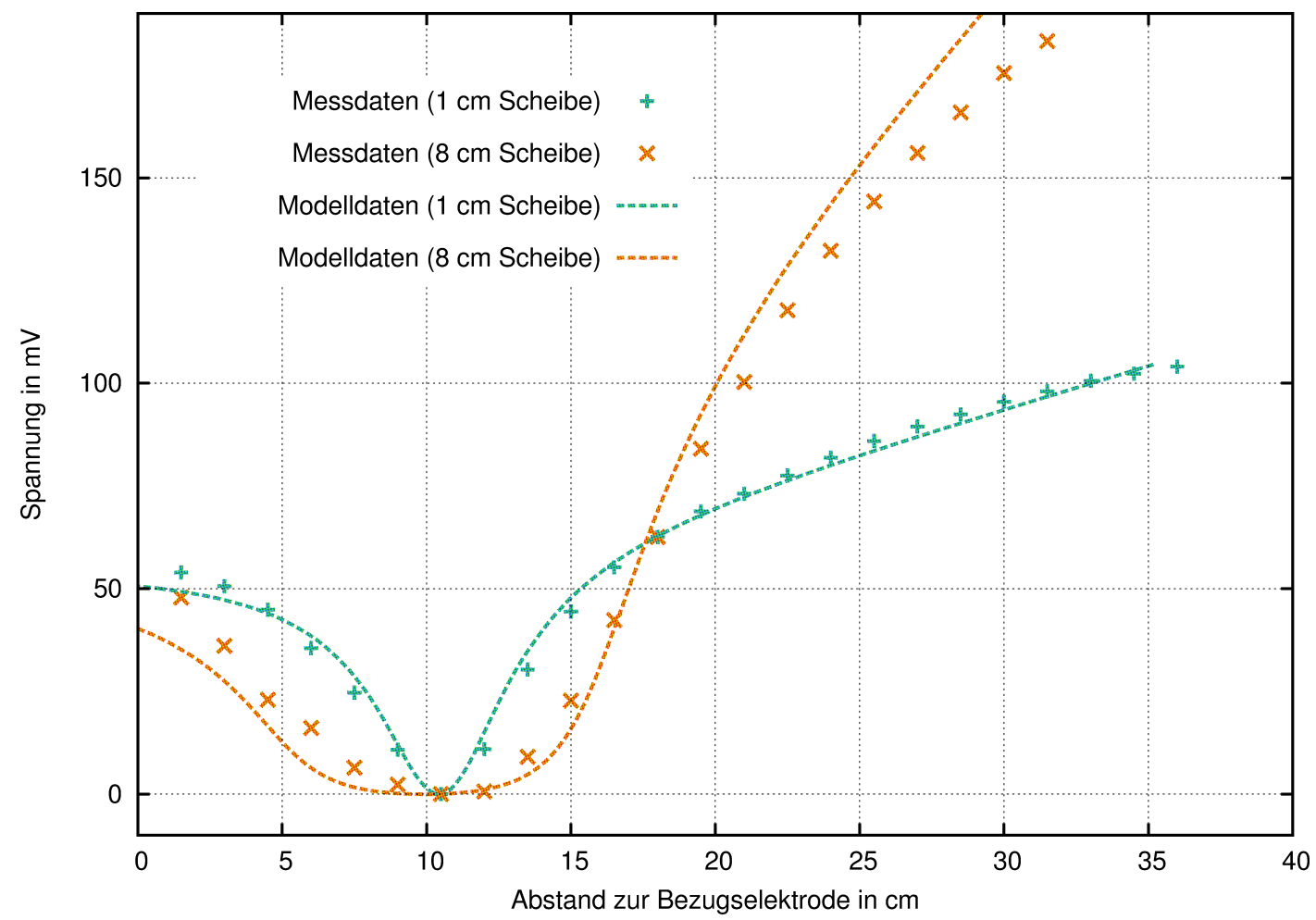

Abbildung 4.6: Ergebnisse des Modellversuchs an zwei unterschiedlich dimensionierten Kupferscheiben $(1 \mathrm{~cm}$ und $8 \mathrm{~cm})$ im elektrolytischen Trog. Die Messdaten werden durch Symbole, die Ergebnisse der numerischen Simulation als gestrichelte Linien dargestellt.

Die Grenzen der Kupferscheiben sind in der Spannungskurve allerdings weniger deutlich zu erkennen, so dass eine Aussage über den Durchmesser der jeweiligen Kupferscheibe höchstens in Relation zueinander möglich ist.

Dieser Befund lässt sich noch deutlicher ausmachen, wenn sich zusätzlich zum veränderten Scheibendurchmesser die Position der Scheibe in Richtung größerer Tiefe ändert. In Abbildung 4.7 und Abbildung 4.8 sind daher Daten aus einer weiteren Modellrechnung mit Kupferscheiben unterschiedlichen Durchmessers in jeweils vier verschiedenen Tiefen auf zwei unter- 


\section{Ergebnisse}

schiedliche Arten dargestellt. Bei diesen Abbildungen werden im Gegensatz zu Abbildung 4.5 und Abbildung 4.6 Spannungsdifferenzen dargestellt. Aus diesem Grund erscheinen hier die Kurven horizontal gespiegelt.

In Abbildung 4.7 werden die beiden unterschiedlich dimensionierten Kupferscheiben bei jeweils vier verschiedenen Tiefen miteinander verglichen. In Abbildung 4.8 werden die verschiedenen Tiefen in jeweils einer Grafik dargestellt. Zunächst lassen die vier Grafiken erkennen, dass unabhängig von der Tiefe der Scheiben der Graph der größeren Scheibe $(8 \mathrm{~cm})$ immer über dem der kleineren liegt. Die Spannungsdifferenzen zwischen den beiden Scheiben liegen hier bei maximal $2 \mathrm{~V}$.

Auffällig ist, dass sich die Kurven in $2 \mathrm{~cm}$ Tiefe für die beiden Scheiben voneinander unterscheiden. Die Spannungskurven für die anderen Tiefenstufen sind nahezu deckungsgleich, sie unterscheiden sich lediglich deutlich hinsichtlich des Spannungsniveaus ihrer absoluten Werte. Eine quantitative Aussage über den absoluten Durchmesser des Objektes im oberflächennahen Bereich ist durch den sehr unscharfen Kurvenverlauf kaum zu treffen.

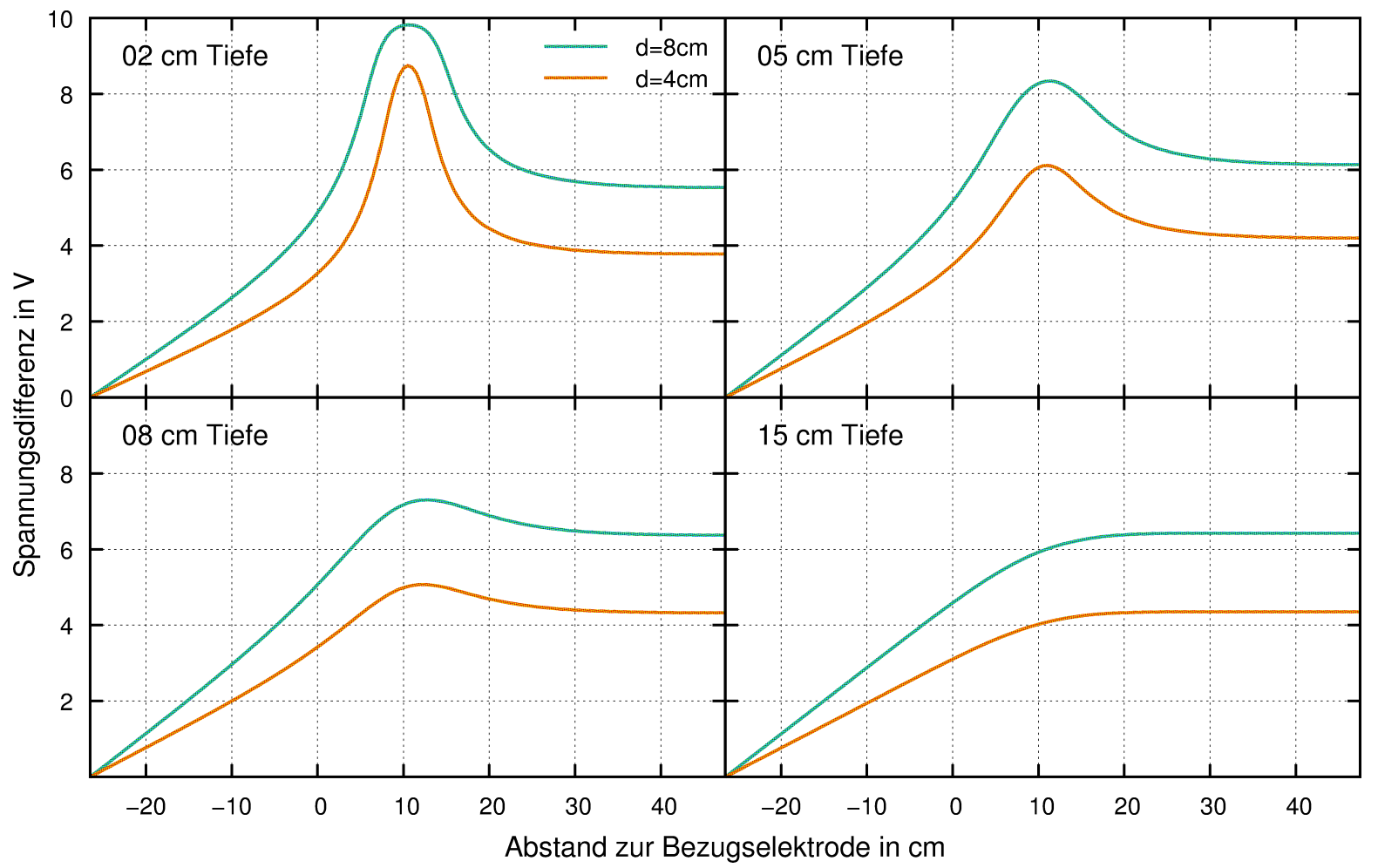

Abbildung 4.7: Vergleich von simulierten Daten zweier Kupferscheiben unterschiedlichen Durchmessers $(\mathrm{d}=8 \mathrm{~cm}$ und $\mathrm{d}=4 \mathrm{~cm})$ in vier verschiedenen Tiefen.

In größerer Tiefe ist dieser Befund noch ausgeprägter. Zudem sind ab einer Tiefe von ca. $5 \mathrm{~cm}$ die Kurvenverläufe beider unterschiedlich dimensionierter Kupferscheiben gering. Eine Einschätzung der Scheibengröße ist somit nicht möglich. Mit zunehmender Tiefe laufen die Graphen der beiden Messungen an zwei Kupferscheiben nahezu parallel. Zur Gegenelektrode (linker Bildbereich) laufen die Kurven stets zusammen. 


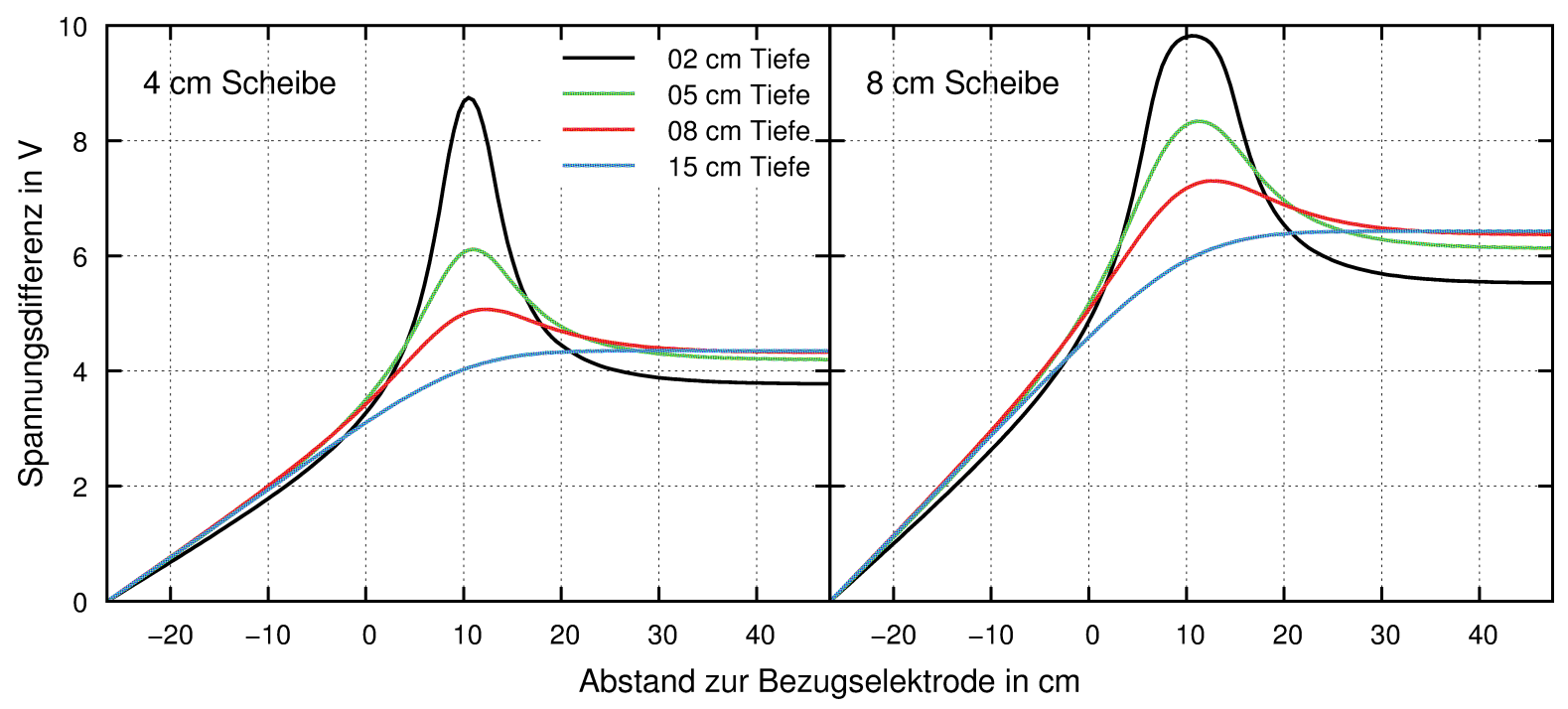

Abbildung 4.8: Vergleich von simulierten Daten zweier Kupferscheiben unterschiedlichen Durchmessers $(\mathrm{d}=8 \mathrm{~cm}$ und $\mathrm{d}=4 \mathrm{~cm})$ in vier verschiedenen Tiefen.

\subsubsection{Feldversuche}

Nachfolgend sind exemplarisch die Ergebnisse einer Messung an einem Amberbaum dargestellt (Abbildung 4.9). Diese stehen stellvertretend für zwei weitere Messungen, die an Fichten (Picea abies) durchgeführt wurden und ähnliche Ergebnisse lieferten.

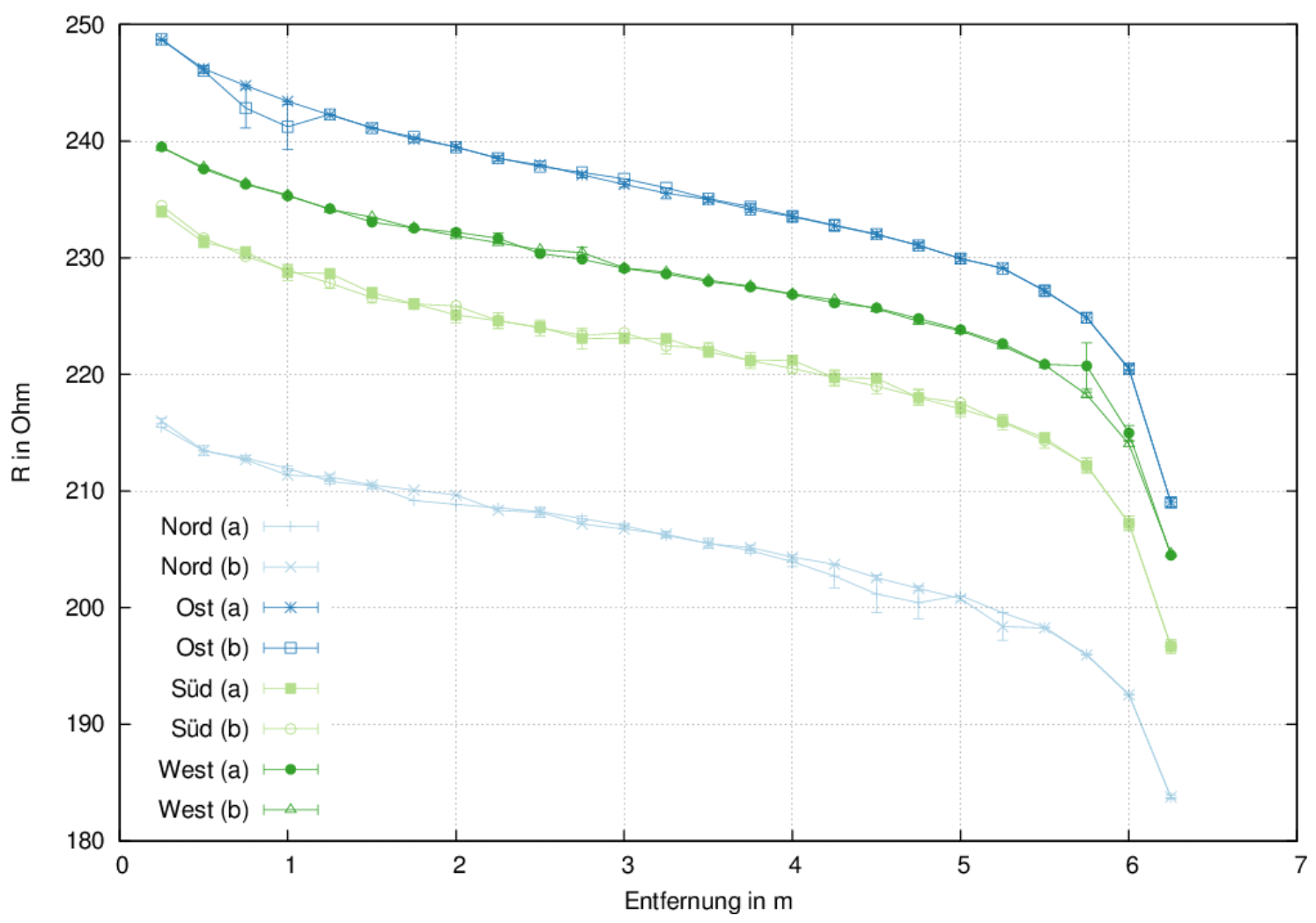

Abbildung 4.9: Bodenwiderstandsmessung an einem Amberbaum entlang vier verschiedener Messlinien. 
Auf der Abszisse ist jeweils der Abstand zum Stamm in Metern, auf der Ordinate der elektrische Widerstand in $\Omega$ dargestellt.

Der Ursprung ist mit der Außenseite des Baumstamms definiert. Die Messungen erfolgten jeweils entlang von vier Messlinien. Jede dieser vier Messungen wurde wiederholt, um die Reproduzierbarkeit ihrer Ergebnisse zu prüfen. Der Fehlerbalken einer jeweiligen Messung ist zusätzlich abgebildet.

Erkennbar ist, dass die vier Messlinien qualtitativ sehr ähnlich erscheinen. Alle vier Kurven fallen zunächst stärker, dann allmählicher ab. Ab einer Entfernung von 5,5 m vom Baum fällt der elektrische Widerstand deutlich rascher ab. Insgesamt liegen die Messwerte zwischen ca. $182 \Omega$ und $249 \Omega$. Insgesamt sind also (2-) 3 Zonen auszumachen.

Die vier Messlinien unterscheiden sich qualtitativ kaum, jedoch absolut sehr deutlich, so dass ein annähernd paralleler Verlauf der vier Graphen zu verzeichnen ist. Bemerkenswert ist weiterhin, dass sich die Ergebnisse reproduzieren lassen. Lediglich geringe Abweichung zweier gleicher Messungen sind auszumachen. Die Fehlerbalken verschmelzen überwiegend mit den Symbolen für die einzelnen Messwerte. Besonders viele Fehler haben jedoch die Messlinien Nord (a) und Nord (b) bspw. im Bereich von $4 \mathrm{~m}-5 \mathrm{~m}$. Hier ist eine deutliche Abweichung der jeweils in gleicher Weise ermittelten Werte zu verzeichnen. An dieser Stelle sind jedoch auch die Fehlerbalken (Nord (a)) am größten.

\subsection{Impedanzspektroskopie}

Zunächst werden die Ergebnisse der Überprüfung des Messsystems veranschaulicht, anschließend die Ergebnisse der Untersuchungen der Randbedingungen. Danach werden die wichtigsten Ergebnisse aus Hautversuch 1 und 2 aufbereitet. Die Hauptversuche gliedern sich in zwei Unterkapitel, bei denen die allgemeinen biologischen und physikalischen Daten Berücksichtigung finden, sowie die modellhafte Betrachtung der gewonnenen Daten erfolgt.

\subsection{1 Überprüfung des Messsystems}

In Abbildung 4.10 werden die Ergebnisse der Überprüfung des Messsystems anhand einer einfachen Testschaltung (vgl. Abschnitt 3.3.1 und Abbildung 3.5 dargestellt (gemessenes Spektrum) und den errechneten Werten dieser Schaltung gegenübergestellt (errechnetes Spektrum).

Zur Überprüfung der Reproduzierbarkeit der gemessenen Daten wurden drei sweeps dargestellt (gemessenes Spektrum). Die drei Kurven liegen übereinander, so dass die Einzelkurven nicht auszumachen sind. Die gemessenen Werte sind reproduzierbar und unterscheiden sich $<1 \%$ voneinander.

Da die Größen der Elemente der Testschaltung bekannt waren, ließ sich das Impedanzspektrum durch Vorwärtsrechnung mit Hilfe des Programms Impedanz (siehe Abschnitt 3.4) generieren. Auf diese Weise lassen sich die wahren Werte mit den gemessenenen Größen vergleichen. In der oberen Grafik wird der Realteil der Impedanz über der Frequenz, in der unteren Grafik der Imaginärteil der Impedanz über der Frequenz dargestellt. 
Bei Betrachtung des Realteils fällt auf, dass beide Kurven in weiten Teilen des Spektrums bis ca. $30 \mathrm{kHz}$ gering voneinander abweichen $(<1 \%)$. Lediglich im höherfrequenten Bereich (ab ca. $30 \mathrm{kHz}$ ) lassen sich etwas größere Abweichungen erkennen (bis $3 \%$ ). Hier sind die gemessenen Werte niedriger als die errechneten.

Die Graphen des Imaginärteils weichen insgesamt deutlicher als beim Realteil der Impedanz voneinander ab. Auffällig ist, dass sämtliche Messwerte hier durchgängig höher als die errechneten Werte sind. Die maximalen Abweichungen betragen hier 7\%. Die Abweichungen sind im hochfrequenten Bereich höher als im niederfrequenten.

Bei sehr vielen sweeps, die im Rahmen der folgenden impedanzspektroskopischen Untersuchungen entstanden sind, ist auffällig, dass bei niedrigen bzw. sehr hohen Frequenzen positive Phasenwinkel $\left(<2^{\circ}\right)$ auftreten. Positive Phasenwinkel würden bedeuten, dass Induktivitäten im Messsystems auftreten (siehe dazu Abschnitt 2.5). Positive Phasenwinkel und die daraus resultierenden (scheinbaren) Induktivitäten werden als Messfehler gewertet (genauere Ausführungen finden sich in Abschnitt 5.3.
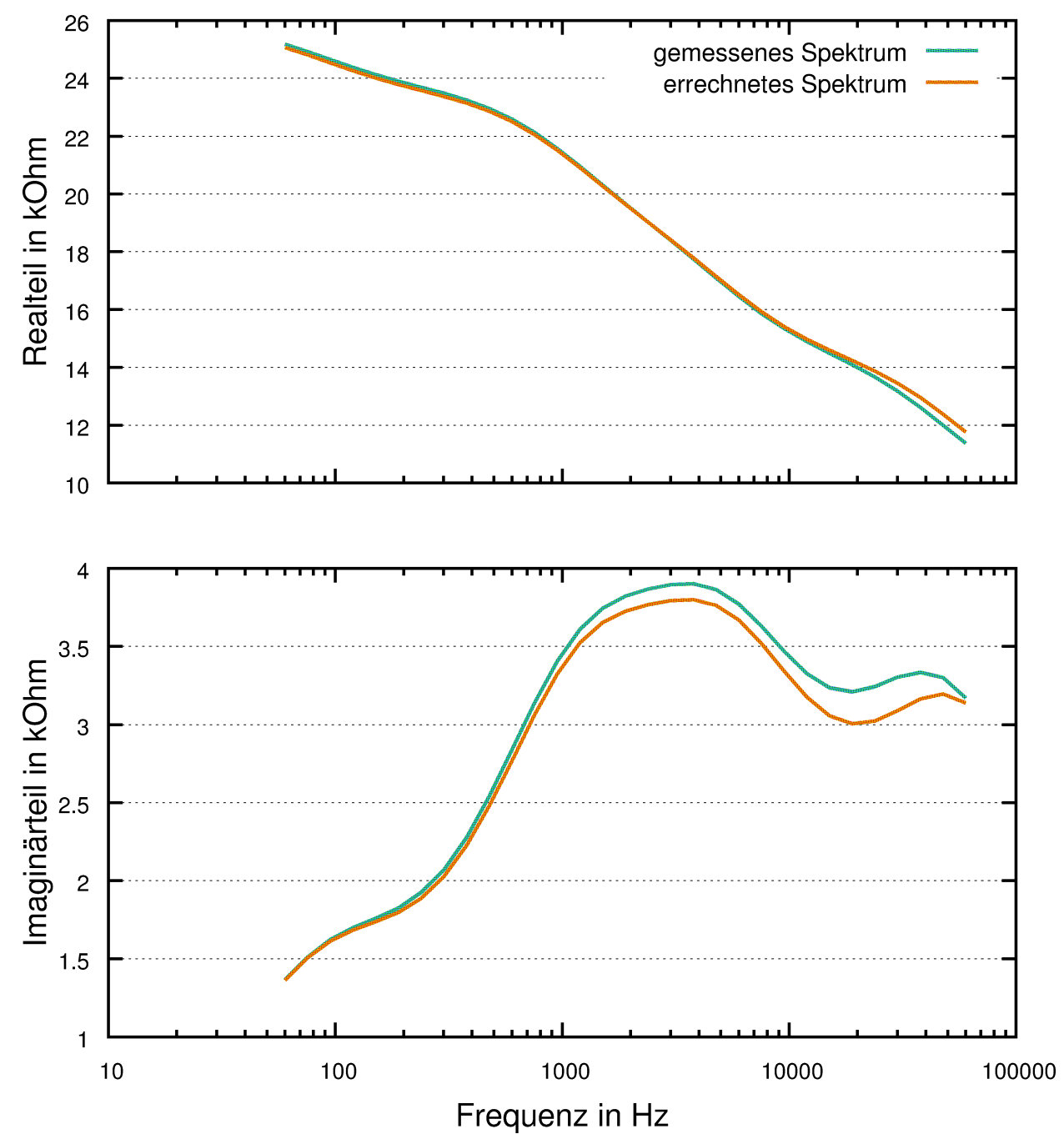

Abbildung 4.10: Vergleich von gemessenen Spektren einer Testschaltung mit dem errechneten Spektrum. 


\subsubsection{Vorversuche zur Untersuchung der Randbedingungen}

\section{Einfluss der Elektrodenwahl und der Elektrodenanordnung}

In Abbildung 4.11 sind die getesteten Nadeln abgebildet, die als Elektroden eingesetzt wurden. Die beiden oberen Nadeln sind vernickelte Stecknadeln, die untere eine Edelstahlkanüle. Es wird deutlich, dass die vernickelten Nadeln im Gegensatz zum Edelstahl (V2A) nicht inert sind. Erkennbar ist dies an den zum Teil massiven Korrosionsfilmen an der Oberfläche der Nadeln. Die Edelstahlkanüle weist dabei visuell keinen Korrosionsschaden auf. Die Silberelektroden wurden zwar nicht näher untersucht, auch diese wiesen aber visuell keine Korrosionsstellen auf.

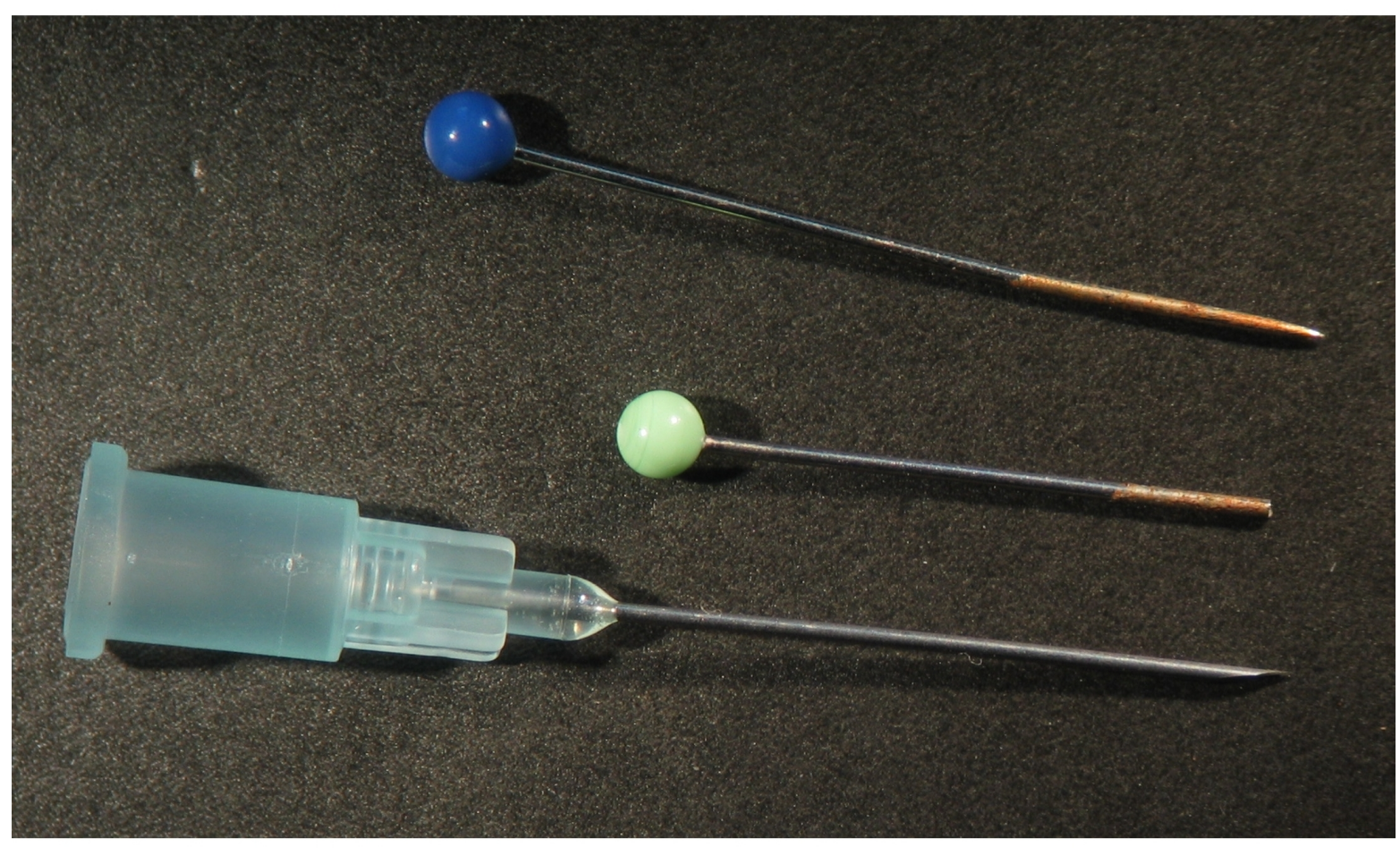

Abbildung 4.11: Visueller Vergleich dreier Nadeln. Oben und Mitte: Zwei gleichartige vernickelte Stecknadeln. Unten: Edelstahlkanüle (V2A).

Darüber hinaus wird in Abbildung 4.12 der Teil einer vergleichbaren Kanüle, der während eines Zeitraumes von vier Wochen im Steckling verblieb (Abbildung 4.12a) mit dem Teil der Kanüle verglichen, der außerhalb des Stecklings im genannten Zeitraum lediglich der umgebenden Luft ausgesetzt war (Abbildung 4.12b).

Die Grafiken sind die Ergebnisse von Röntgenfluoreszensanalysen (RFA), die mit Hilfe eines Rasterelektronenmikroskops entstanden sind. Die Abbildungen zeigen die relativen Konzentrationen der chemischen Elemente der Kanülenoberfläche auf der Ordinate.

Bei der Röntgenfluoreszensanalyse werden Elektronen von chemischen Elementen angeregt und aus ihren Schalen herausbefördert. Der so frei gewordene Platz auf einer Schale wird von Elektronen höherer Schalen eingenommen. Bei diesem Elektronensprung wird Energie frei, die gemessen werden kann. Diese Energie ist abhängig von der Kernladungszahl. Da sich chemische 
Elemente hauptsächlich durch ihre Kernladungszahl unterscheiden, können diese aufgrund von Energiedifferenzen identifiziert werden. In Abbildung 4.12 ist diese gemessene Energie auf der Abszisse in Kiloelektronenvolt (keV) aufgetragen. Die Buchstaben Ka (eigentlich $\mathrm{K} \alpha$ ) stehen für den Sprung von Elektronen aus der K- in die M-Schale eines Elements. In den Beschriftungen der Grafiken taucht neben dem chemischen Element deshalb immer das Suffix Ka auf. Weiterführende Erläuterungen zur RFA findet sich bspw. bei Agarwal u. a. 1979).

Die Konzentrationen von Eisen (Fe), Chrom (Cr), Nickel (Ni) und Silizium (Si) bleiben in beiden Fällen auf ähnlichen Niveaus. Die Konzentration von Sauerstoff $(\mathrm{O})$ und besonders von Kohlenstoff (C) sind jedoch verschieden. Bei dem Teil der Elektrode, der im Steckling steckte, ist die Kohlenstoffkonzentration etwas erhöht (obere Grafik, linke Bildseite). Das Sauerstoffniveau ist hier hingegen leicht niedriger als außerhalb des Stecklings.

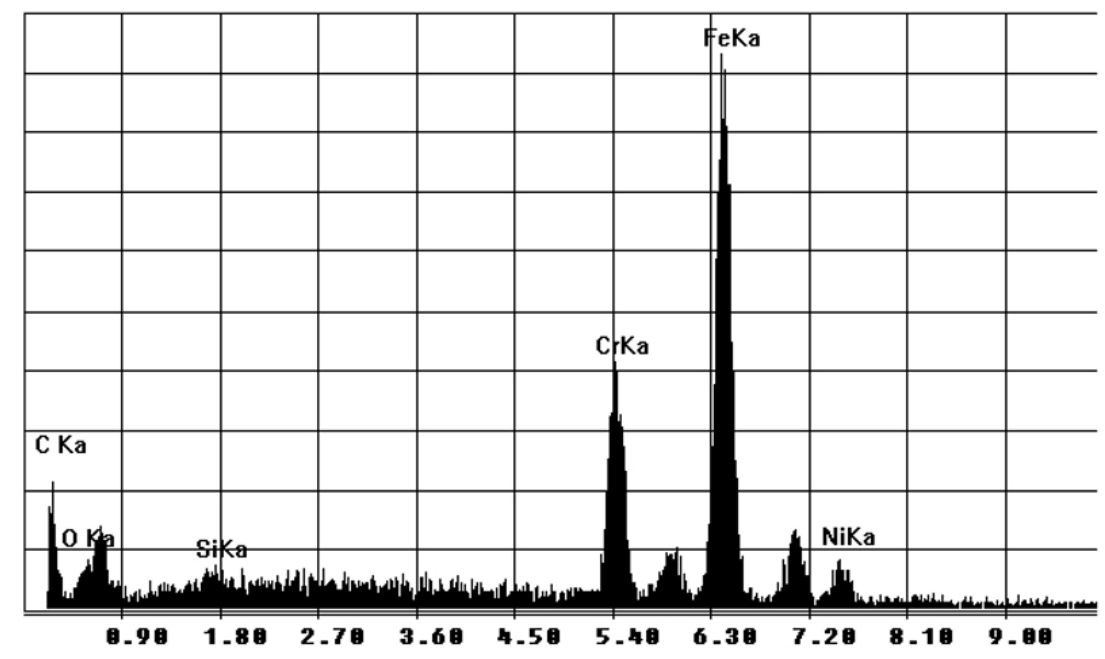

(a)

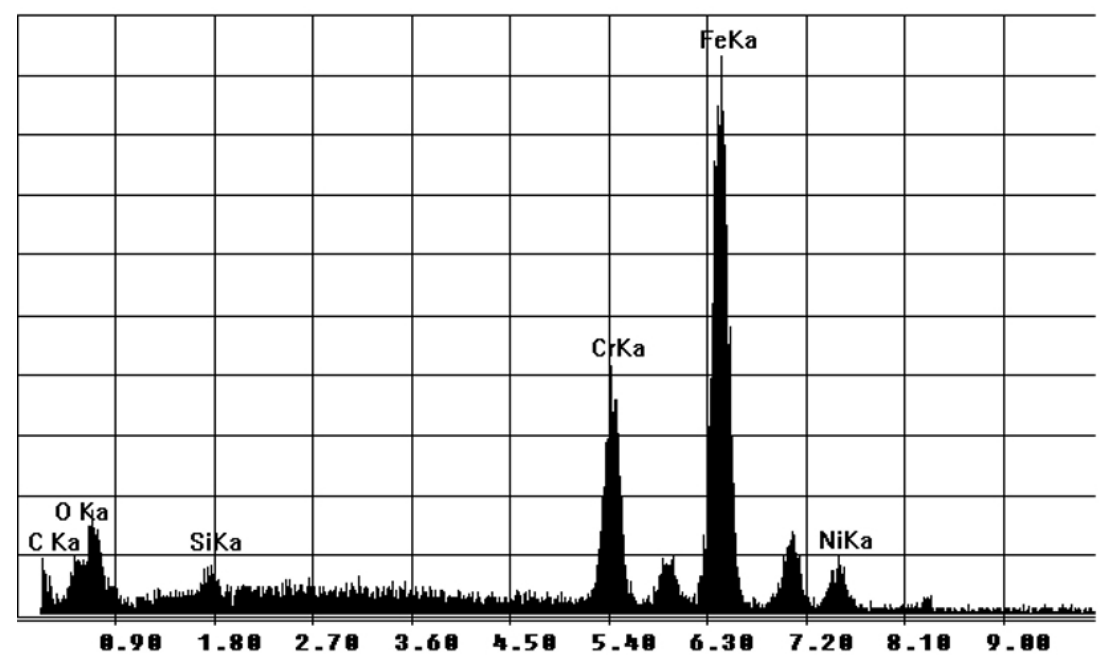

(b)

Abbildung 4.12: Analyse der Edelstahlkanülenoberfläche mit dem Rasterelektronenmikroskop aus dem Bereich der Kanüle, der im Steckling steckte (a) und aus dem Bereich außerhalb des Stecklings (b). 
Insgesamt lässt diese Grafik erkennen, dass der Kontakt der Elektrode mit dem Steckling nur zu geringen Veränderungen der chemischen Zusammensetzung an der Kanülenoberfläche führt. Die Fotos von den Oberflächen der beiden Bereiche der Elektroden unter dem REM zeigen auch visuell keine Unterschiede (vgl. Abbildung A.1).

Weiterhin wurden die impedanzspektroskopischen Versuche mit vergleichbaren V2A-Kanülen an Weidenstecklingen in dem in Abbildung 3.8 skizzierten Versuchsaufbau durchgeführt. In Abbildung 4.13 sind die Ergebnisse von vier unterschiedlichen Messungen in zwei verschiedenen Messkonfigurationen (Zwei- und Vierpunktmessungen) und jeweils zwei verschiedenen Elektrodenmaterialien (Edelstahl und Silber) in der komplexen Ebene dargestellt.

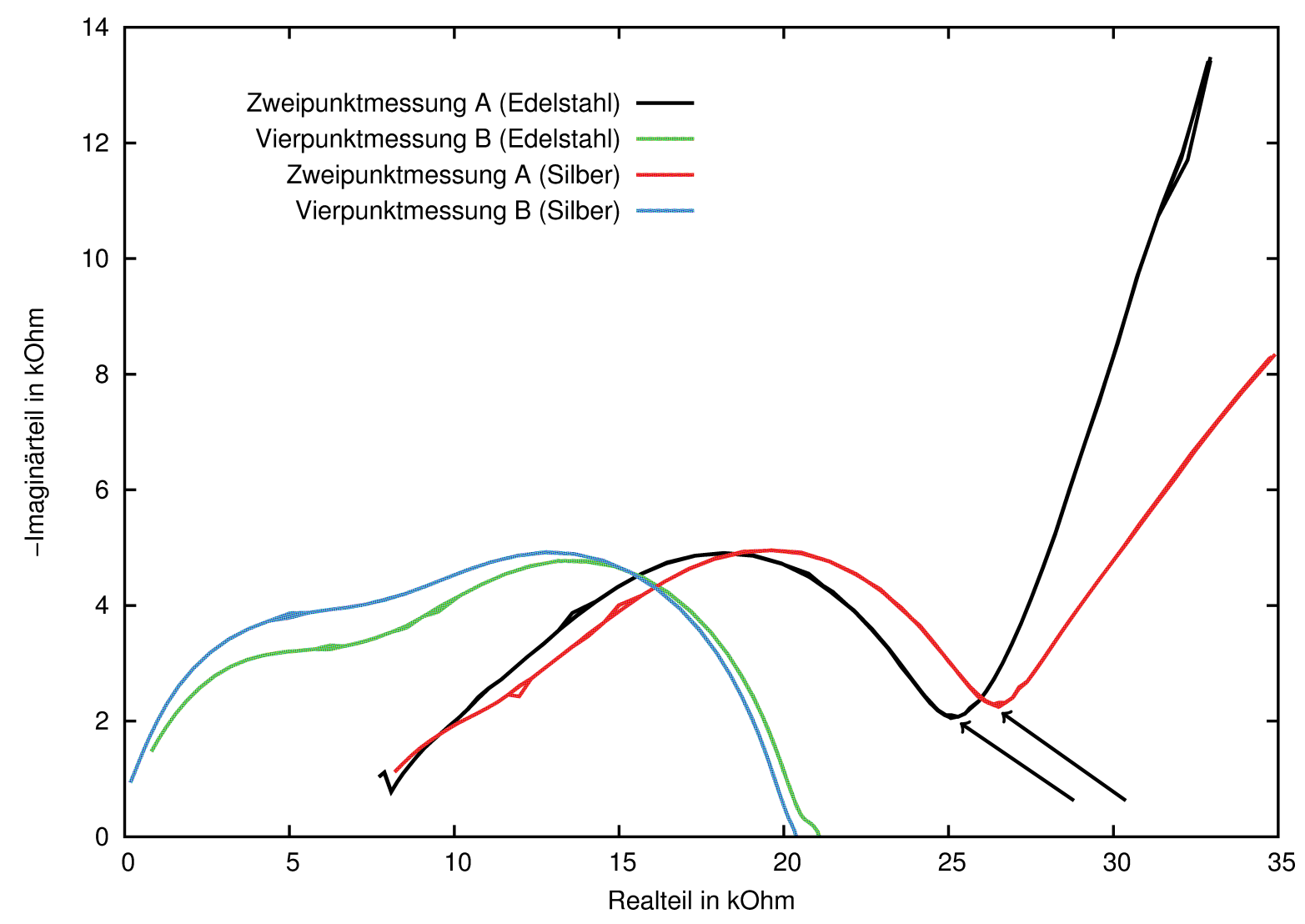

Abbildung 4.13: Vergleich von impedanzspektroskopischen Messungen in vier verschiedenen Elektrodenkonfigurationen an einem Weidensteckling in der komplexen Ebene. Weitere Erläuterungen finden sich im Text.

Vergleicht man zunächst die Messungen, die in den beiden unterschiedlichen Messkonfigurationen durchgeführt wurden (Zweipunktmessung A und Vierpunktmessung B), so fällt auf, dass sich die absoluten Werte deutlich unterscheiden. Besonders stark ist der Unterschied beim Realteil der Impedanz. Die Zweipunktmessungen, sowohl bei der Silber-, als auch bei der Edelstahlelektrode, weisen höhere Realteile auf. Die Imaginärteile der Zweipunktmessungen A unterscheiden sich im hochfrequenten Bereich (linker Teilbereich der Grafik) kaum von denen der Vierpunktmessungen B. Im niederfrequenten Bereich liegen die Imaginärteile der Zweipunktmessungen deutlich über denen der Vierpunktmessung (rechter Teilbereich der Grafik 
ab $\nwarrow)$. Der größte Effekt ist beim Imaginärteil der Edelstahlelektrode erkennbar $(<-13 \mathrm{k} \Omega)$. Die beiden Messkonfigurationen unterscheiden sich nicht nur von den absoluten Werten, sondern auch im Verlauf der Graphen.

Beim Vergleich der Edelstahl- und der Silberelektrode fällt jedoch auf, dass sich die Verläufe der beiden Messungen mit gleicher Messkonfiguration stark ähneln. Die absoluten Werte der beiden gleichen Konfigurationen unterscheiden sich kaum. Insgesamt sind die Imaginärund Realteile der Silberelektrodenmessung hochohmiger als die der Edelstahlelektrode. Diese Effekte lassen sich besonders deutlich im Bode-Diagramm in Abbildung 4.14 verdeutlichen. In diesen Diagrammen werden die beiden Elektrodenmaterialien, die in den beiden Hauptversuchen verwendet werden, direkt miteinander bei verschiedenen Elektrodenkonfigurationen verglichen. Links sind die Messungen mit Edelstahlelektroden dargestellt, rechts jene mit Silberelektroden. Die oberen Grafiken zeigen jeweils den Realteil, die unteren den Imaginärteil der Impedanz über der Frequenz. Es werden jeweils fünf verschiedene Messungen mit unterschiedlichen Elektrodenkonfigurationen veranschaulicht.
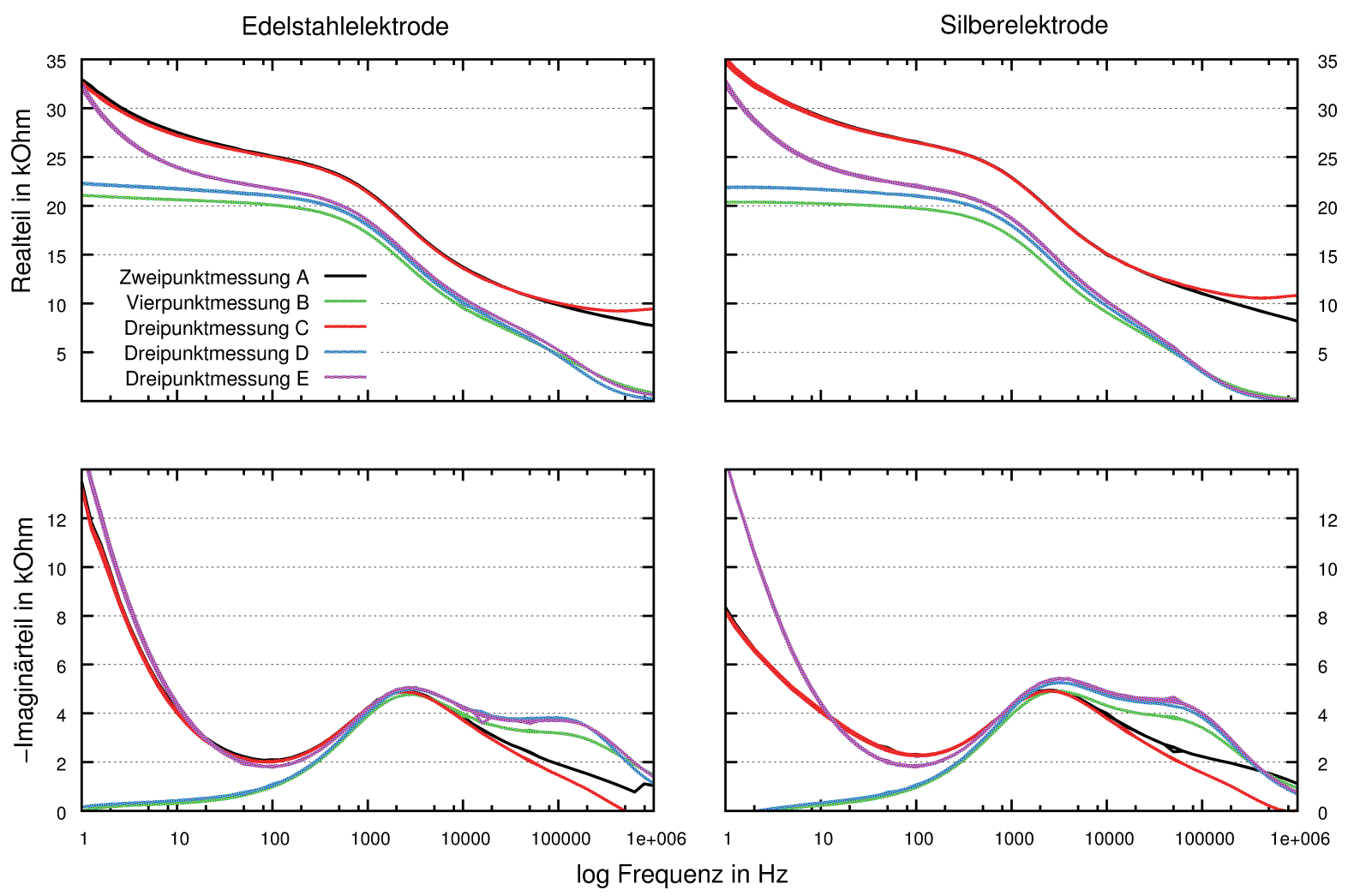

Abbildung 4.14: Vergleich von Real- und Imaginärteil der Impedanz über der Frequenz bei verschiedenen Elektrodenkonfigurationen und zwei verschiedenen Elektrodenmaterialien an einem Weidensteckling. Links Edelstahlelektroden, rechts versilberte Kupferelektroden.

Neben den bereits in Abbildung 4.13 in der komplexen Ebene dargestellten Zwei- und Vierpunktmessungen, sind hier zusätzlich drei Dreipunktmessungen (C, D und E) abgebildet (siehe Tabelle 3.1]. Auch beim Vergleich der Dreipunktmessungen zeigt sich, dass die Ergebnisse von Edelstahl- und Silberelektrode ähnlich sind und die beiden Graphen etwa parallel verschoben 
sind. Lediglich geringe Abweichungen bei gleicher Elektrodenkonfigurationen aber anderem Elektrodenmaterial sind festzustellen.

Weiterhin wird deutlich, dass die Vierpunktmessungen B und die Dreipunktmessungen D $\mathrm{zu}$ ähnlichen Ergebnissen führen. Zwar gibt es Abweichungen bei den absoluten Werten, jedoch weist der Verlauf der beiden Graphen eine hohe Ähnlichkeit auf. Die Dreipunktmessung ist dabei unwesentlich hochohmiger als die Vierpunktmessung. Dieser Befund macht sich bei niedrigen Frequenzen im Realteil der Impedanz bemerkbar. Diese Erkenntnisse gelten sowohl für die Edelstahl-, als auch für die Silberelektroden.

Bei Betrachtung des Imaginärteils zeigen sich im Bereich von ca. $5000 \mathrm{~Hz}$ geringe Abweichungen zwischen den Messungen mit unterschiedlichen Elektronenkonfigurationen. Auffällig ist jedoch der Bereich von $1 \mathrm{~Hz}-100 \mathrm{~Hz}$. Hier kommt es bei den Messungen $\mathrm{C}$ und E, wie bei den Zweipunktmessungen A gesehen, zu deutlich höheren Imaginärteilen als bei der Vierpunktmessung B und der Dreipunktmessung D. Betrachtet man den Realteil über der Frequenz, so wird deutlich, dass sich dieser ebenfalls im genannten Frequenzbereich unterscheidet. Im niederfrequenten Bereich sind die Realteile der Zweipunktmessung A und der Dreipunktmessungen C und E deutlich erhöht. Besonders die Zweipunktmessung A und die Dreipunktmessung $\mathrm{C}$ haben dabei über den gesamten Frequenzverlauf sehr ähnliche absolute Werte und unterscheiden sich deutlich im Realteil von den übrigen. Lediglich im höheren Frequenzbereich (ca. $100 \mathrm{kHz}$ ) kommt es bei den Zweipunktmessungen A und den Dreipunktmessungen C zu Unterschieden. Dabei liegen die Realteile der Dreipunktmessungen C über denen der Zweipunktmessungen A.

\section{Einfluss der Leitfähigkeit der Nährlösung}

In Abbildung 4.15 sind Real- und Imaginärteil der Messungen an zwei ausgewählten Weidenstecklingen in drei verschieden leitfähigen Nährlösungen stellvertretend für 15 Stecklinge über der Frequenz dargestellt. Die Ergebnisse an 13 weiteren Stecklingen sind im Anhang in den Abbildungen A.2 bis A.5 zu sehen.

Bei Betrachtung des Realteils fällt auf, dass bei steigender Frequenz der Realteil der Impedanz sinkt, wie bereits bei den vorigen impedanzspektroskopischen Versuchen gesehen. Dieser Trend lässt sich bei allen drei Nährlösungen feststellen. Dieses Ergebnis zeigt sich auch bei den übrigen Stecklingen dieses Versuches. Der Imaginärteil der Impedanz ist bei diesen Stecklingen bei ca. $1000 \mathrm{~Hz}$ am größten.

Vergleicht man die Ergebnisse des Realteils der Impedanz bei einer bestimmten Frequenz und unterschiedlichen Leitfähigkeiten miteinander, so fällt auf, dass sich die Messwerte bei einer Leitfähigkeit von $54 \mu \mathrm{S} \mathrm{cm}^{-1}$ von den anderen beiden deutlich unterscheiden. In den meisten Fällen dieses Kollektivs liegen diese Werte deutlich über denen der anderen beiden Leitfähigkeiten. Ausnahmen bilden bspw. Steckling 4 und 14. Bei einer Frequenz von ca. $5000 \mathrm{~Hz}$ ist der Realteil der Impedanz bei einer Leitfähigkeit von $54 \mathrm{\mu S} \mathrm{cm}^{-1}$ um ca. $50 \%$ größer als der Realteil der Impedanz bei einer Leitfähigkeit von $245 \mu \mathrm{S} \mathrm{cm}^{-1}$. Vergleicht man die Werte des Realteils der Impedanz bei einer Leitfähigkeit von $245 \mu \mathrm{S} \mathrm{cm}^{-1}$ mit $508 \mu \mathrm{S} \mathrm{cm}^{-1}$, so fällt auf, dass die Unterschiede nur sehr gering sind. Als Trend ist erkennbar, dass die Werte der höheren Leitfähigkeit unter denen der niedrigeren Leitfähigkeit liegen. Bei bestimmten Steck- 
lingen und Frequenzen gibt es jedoch Abweichungen von diesem Befund. Besonders groß ist die Abweichung im unteren Frequenzbereich dieses Kollektivs (z. B. Steckling 2 bei $10 \mathrm{~Hz}$ und $100 \mathrm{~Hz})$.

Obwohl also die Leitfähigkeit der Nährlösung von $245 \mu \mathrm{S} \mathrm{cm}^{-1} \mathrm{zu} 508 \mu \mathrm{S} \mathrm{cm}^{-1}$ mehr als verdoppelt wurde, zeigt sich dieser Effekt kaum im Realteil der Impedanz.

Der Imaginärteil der Impedanz steigt zunächst bis ca. $1000 \mathrm{~Hz}$ an und fällt anschließend wieder ab. Bei den Imaginärteilen der Impedanz bei einer Leitfähigkeit von $54 \mathrm{\mu S} \mathrm{cm}^{-1}$ fällt auf, dass der Kurvenverlauf im höheren Frequenzbereich (> $20000 \mathrm{~Hz})$ von denen der anderen beiden Nährlösungen nicht nur von ihren absoluten Werten, sondern auch vom Verlauf des Graphen deutlich abweicht. Im Bereich von $(500000 \mathrm{~Hz})$ ist ein zweiter deutlicher Hochpunkt zu erkennen, während ein solcher bei einer Leitfähigkeit von $508 \mu \mathrm{S} \mathrm{cm}^{-1}$ nicht vorhanden ist. Der Imaginärteil bei unterschiedlichen Stecklingen und Leitfähigkeiten zeigt ebenso den Effekt, dass sich die Werte bei der Leitfähigkeit von $54 \mu \mathrm{S} \mathrm{cm}^{-1}$ von den anderen abheben. Die Relation ist jedoch bei bestimmten Frequenzen (z. B. $10000 \mathrm{~Hz}$ ) geringer. Grundsätzlich liegen die Imaginärteile der Messungen mit einer Leitfähigkeit von $54 \mu \mathrm{S} \mathrm{cm}^{-1}$ über denen der anderen beiden.
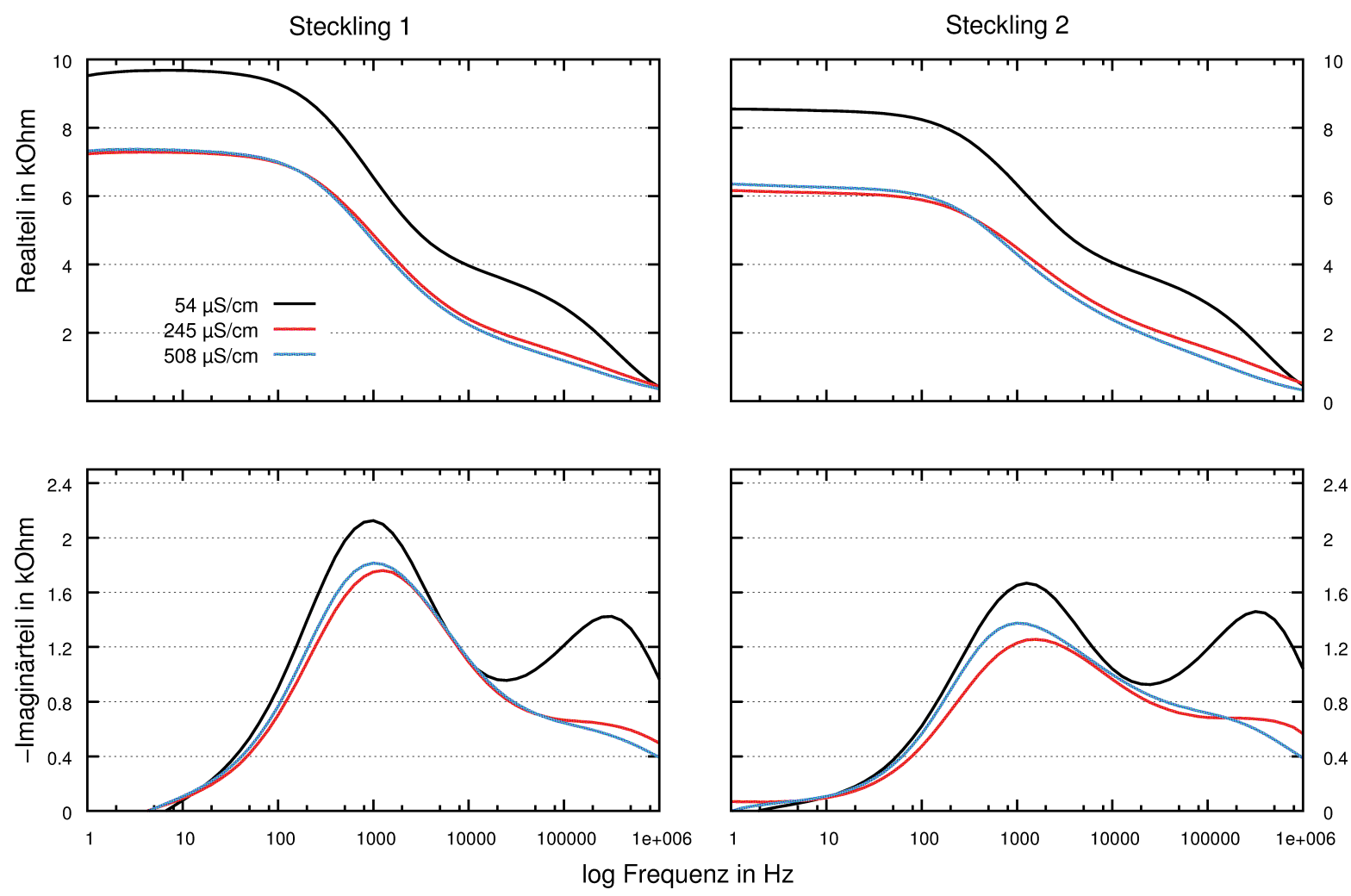

Abbildung 4.15: Ergebnisse des Nährlösungsversuches. Dargestellt werden exemplarisch 2 Stecklinge (Steckling 1, links und Steckling 2, rechts) bei drei verschieden leitfähigen Nährlösungen. Oben befinden sich die Realteile, unten die Imaginärteile der Impedanz. 


\section{Einfluss des Wasserstandes}

In Abbildung 4.16 ist der Realteil der Impedanz über der Eintauchtiefe der vier untersuchten Stecklinge dargestellt. Ausgewählt wurden hier vier verschiedene Frequenzen $(1 \mathrm{~Hz}, 100 \mathrm{~Hz}$, $1 \times 10^{3} \mathrm{~Hz}, 2,2 \times 10^{3} \mathrm{~Hz}$ und 4,6 $\times 10^{3} \mathrm{~Hz}$ ). Der höhere Frequenzbereich (> ca. $50 \mathrm{kHz}$ ) wurde hier nicht beachtet, da hier negative Werte beim Realteil der Impedanz auftauchen. Erkennbar ist, dass eine veränderte Eintauchtiefe einen großen Effekt auf den Realteil der Impedanz hat. Die Unterschiede der Startmesswerte (Nullmarke) zu den Werten der Eintauchtiefe von $78 \mathrm{~mm}$ machen je nach betrachteter Frequenz eine Differenz von ca. 25\%-65\% aus.

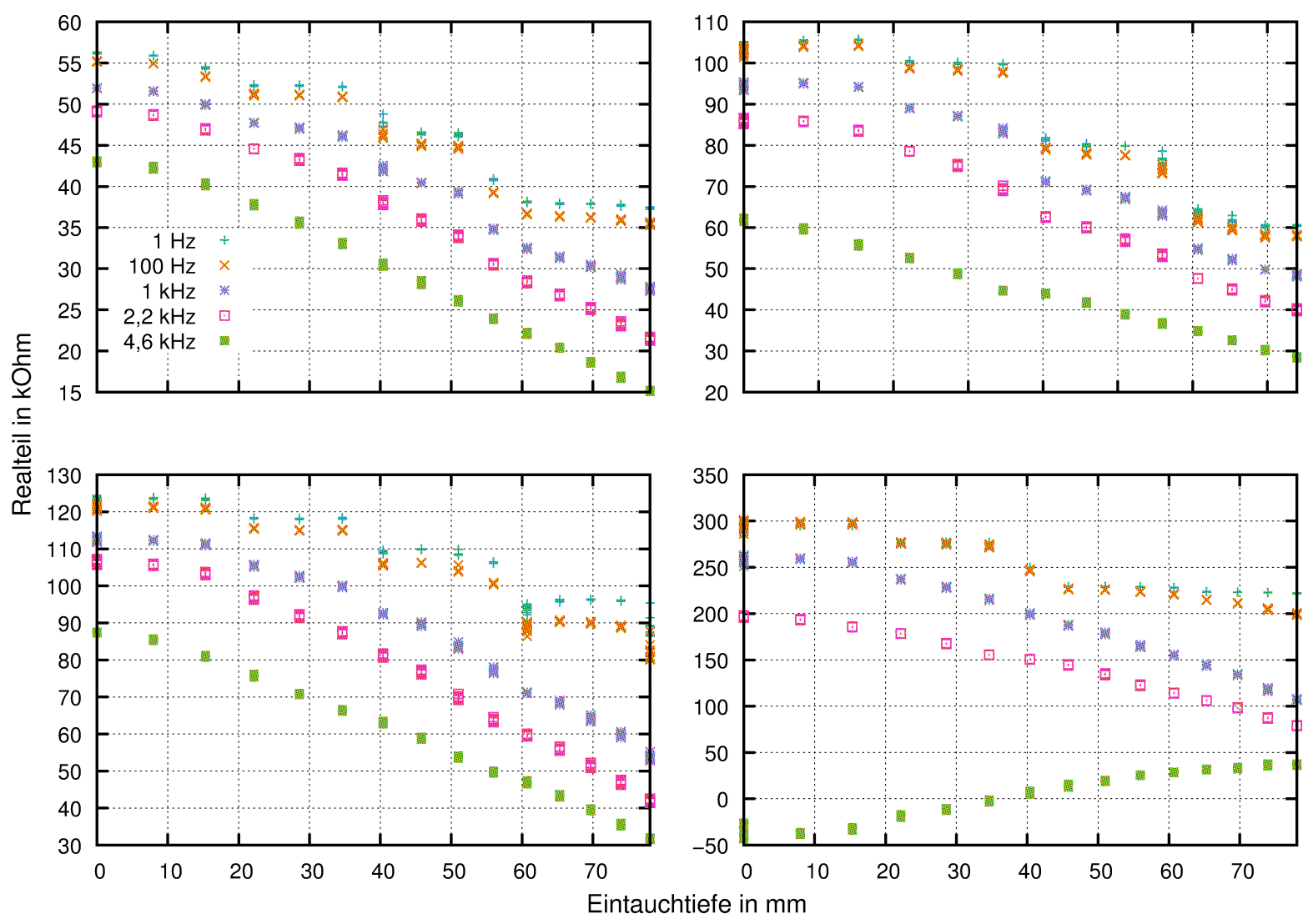

Abbildung 4.16: Veränderung des Realteils der Impedanz bei sukzessiver Änderung der Eintauchtiefe. Dargestellt sind Werte bei 5 ausgewählten Frequenzen von $1 \mathrm{~Hz}-$ $4,6 \mathrm{~Hz}$ von Steckling A (9,31 cm Durchmesser, oben links), B (6,64 cm Durchmesser oben rechts), C (6,07 cm Durchmesser, unten links), D (3,88 cm Durchmesser, unten rechts).

Der Zusammenhang zwischen Eintauchtiefe und Realteil der Impedanz ist negativ und mit annähernd linearem Trend. Durch den linearen Zusammenhang zwischen beiden untersuchten Parametern kann postuliert werden, dass eine Veränderung der Eintauchtiefe von einem Zentimeter ca. 8,3\%-3,2\% hinsichtlich des Realteils ausmacht. Die Linearität des Zusammenhanges zeigt sich sowohl bei niedrigen, als auch höheren Frequenzen bis $100 \mathrm{kHz}$ und bei jedem der 4 untersuchten Stecklinge. Eine Ausnahme bei dem hier untersuchten Kollektiv bildet Steckling D bei einer Frequenz von 4,6 kHz. Hier ist ein annähernd positiver linearer Trend zwischen den 
untersuchten Parametern zu erkennen.

Besonders bemerkenswert erscheint der Bereich im oberen Kilohertzbereich. Hier ist die Differenz des Realteils in den Höhenstufen von $0 \mathrm{~mm}$ zu $78 \mathrm{~mm}$ am größten. Dies zeigt sich zudem in Abbildung 4.17. In dieser Abbildung sind die Messwerte der bereits in Abbildung 4.16 erwähnten vier Stecklinge in der komplexen Ebene bei den 15 (Steckling B nur 14) verschiedenen Eintauchtiefen dargestellt. Im mittleren Frequenzbereich ist eine starke Verkleinerung der „Halbkreise“ bei zunehmender Eintauchtiefe erkennbar.

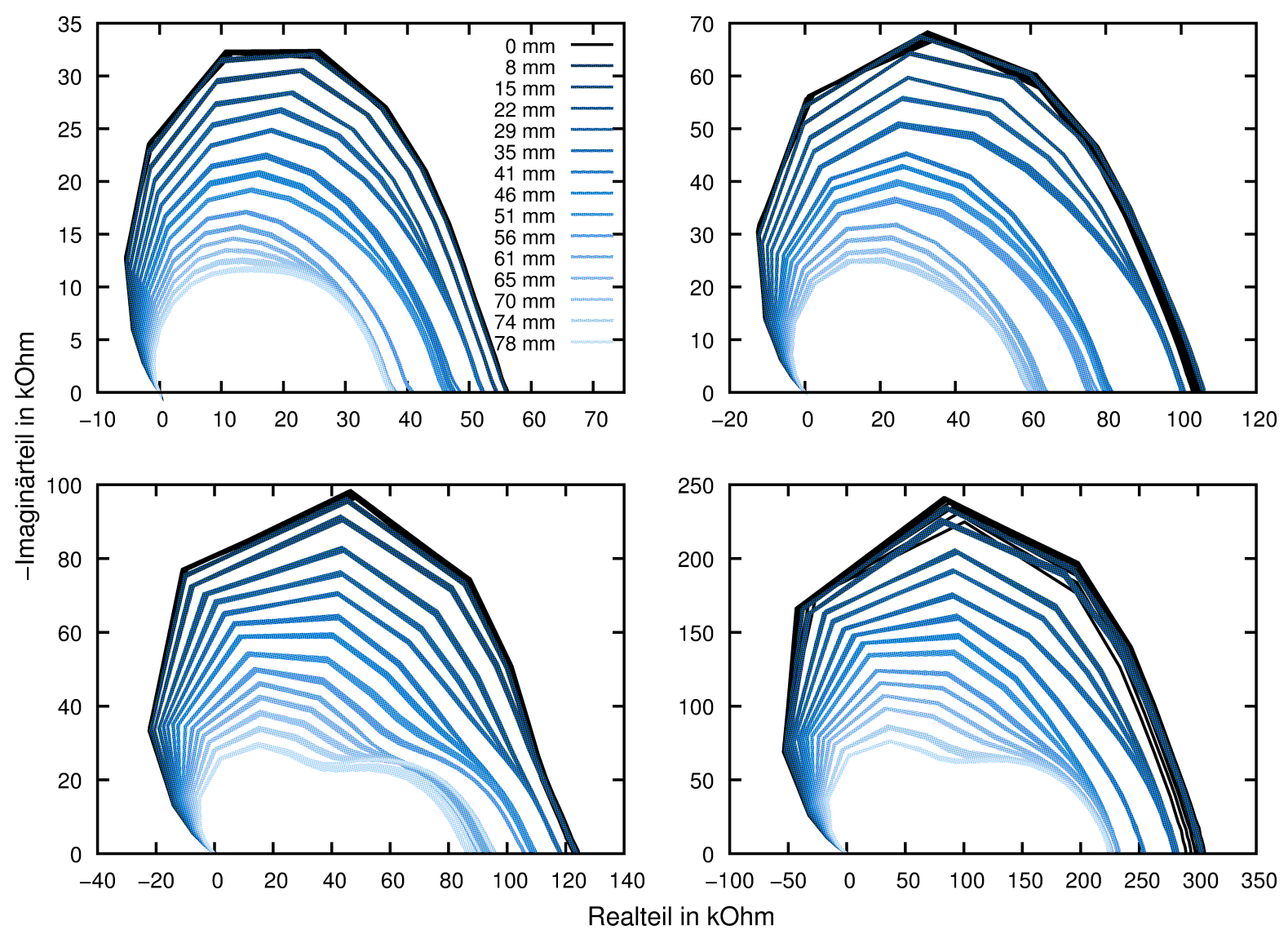

Abbildung 4.17: Darstellung des Imaginärteils der Impedanz über dem Realteil der Impedanz von Steckling A (oben links), B (oben rechts), C (unten links) und D (unten rechts) bei verschiedenen Eintauchtiefen.

Hier ist der Einfluss des veränderten Wasserstandes auf Real- und Imaginärteil der Impedanz am deutlichsten. Zudem wird die Verschiebung der Halbkreise nach rechts und die Verkleinerung der Halbkreise, sprich die Abnahme der Real- und Imaginärteile insgesamt, deutlich. Ebenfalls erwähnenswert erscheint der hochfrequente (linke) Bereich. Hier liegen die Realteile der Impedanz zum Teil im negativen Bereich, da die Phasenwinkel hier positiv sind. Je tiefer der Steckling jedoch eintaucht, desto kleiner ist der Frequenzbereich mit negativen Werten. Dieser Effekt tritt noch deutlicher bei geringen Eintauchtiefen und dünneren Stecklingen auf (z. B. Steckling D). Erkennbar ist weiterhin, dass sich der Verlauf einiger Kurven, insbesondere bei Steckling C und D, deutlich von anderen Kurven unterscheidet. Wohingegen die Impedanzkurven bei den Stecklingen A und B halbkreisähnliche Verläufe ausbilden, treten annähernd 


\section{Ergebnisse}

zweigipflige Kurvenverläufe bei Steckling C ab einer Eintauchtiefe $51 \mathrm{~mm}$ und bei Steckling D ab einer Eintauchtiefe ab $>65 \mathrm{~mm}$ auf.

\section{Einfluss der Temperatur}

In Abbildung 4.18 ist ein ausgewählter Zeitabschnitt von impedanzspektroskopischen Untersuchungen an einem Weidensteckling über der Zeit dargestellt. Betrachtet wurde der Zeitraum vom 31.08.2011 um 12:00 Uhr bis zum 05.09.2011 um 12:00 Uhr. In den Einzelgrafiken werden sechs unterschiedliche Frequenzen zwischen $3,162 \mathrm{~Hz}$ und 316,200 kHz unterschieden. Auf der Abszisse ist die Zeit, auf der 1. Ordinate der Realteil der Impedanz der jeweiligen Frequenz, auf der 2. Ordinate (in der ersten Einzelgrafik) zusätzlich die Temperatur in ${ }^{\circ} \mathrm{C}$ aufgetragen. Der Steckling wurde für diesen Versuch zwei verschiedenen Phasen ausgesetzt.

Im ersten Zeitfenster (in der linken Hälfte der Grafik bis zum 02.09.2011 um 19:00 Uhr) ist die Dauerlichtphase dargestellt. Die Temperatur pendelt sich mit geringer Streuung zwischen $20^{\circ} \mathrm{C}$ und $20,5^{\circ} \mathrm{C}$ ein.

In der rechten Grafikhälfte wird die Phase mit intervallweise wechselnden Lichtverhältnissen dargestellt (farblich hinterlegter Bereich, ab 02.09.2011 um 19:00 Uhr). In diesem Zeitfenster folgte stets eine Dunkelphase (19:00 Uhr-7:00 Uhr) auf eine Lichtphase (7:00 Uhr-19:00 Uhr). Diese Phasen werden im weiteren Verlauf auch als Nacht (Dunkelphase) und Tag (Lichtphase) bezeichnet.

Der Realteil der Impedanz zeigt während der Dauerlichtphase (erstes Zeitfenster) je nach Frequenz einen Schwankungsbereich von ca. 1,1\%-7\% des Maximalwertes.

Auffälligkeiten im Verlauf des Realteils sind im ersten Zeitfenster am Anfang (31.08.2011 um 12:00 Uhr bis 31.08.2011 um ca. 21:00 Uhr) und am Ende bzw. am Anfang des zweiten Zeitfensters (Phase mit wechselnder Beleuchtung) (02.09.2011 um ca. 11:00 Uhr bis 02.09.2011 um ca. 22:00 Uhr) erkennbar. Im erstgenannten Zeitraum sinkt der Realteil bis ca. 15:00 Uhr rasch ab und steigt anschließend bis ca. 21:00 Uhr deutlich langsamer wieder an.

Am 02.09.2011 um ca. 11:00 Uhr sinkt der Realteil bis ca. zum 02.09.2011 um ca. 16:30 Uhr erneut deutlich ab und steigt anschließend bis zum 02.09.2011 um ca. 22:00 Uhr an. Die Abnahme und die anschließende Zunahme am 02.09.2011 macht sich bei höheren Frequenzen besonders bemerkbar. Bei den Frequenzen der ersten beiden Teilgrafiken ist der erwähnte Verlauf kaum zu erkennen. Am 31.08.2011 ist der auffällige Verlauf bei allen betrachteten Frequenzen erkennbar.

In der ersten Dunkelphase vom 02.09.2011 um 19:00 Uhr bis 03.09.2011 um 7:00 Uhr ist erkennbar, dass sich die Kammer allmählich bis zum Tiefststand von ca. $18,8^{\circ} \mathrm{C}$ abkühlt. Sobald um 7:00 Uhr die Beleuchtung einsetzt, steigt die Temperatur auf max. $20,75^{\circ} \mathrm{C}$. Diese Periodizität ist auch in den folgenden Tagen und über den gesamten Verlauf dieses Experimentes erkennbar.

Im Gegensatz zur absinkenden Temperatur in der Dunkelphase, zeigt sich beim Realteil ein deutlicher Anstieg der Widerstandswerte. Dieser gegenläufige Effekt tritt umgekehrt auch beim Anstieg der Temperatur während der dauerhaften Beleuchtung auf. Während die Temperatur nach Einschalten der Beleuchtung steigt, sinkt der Realteil der Impedanz. 


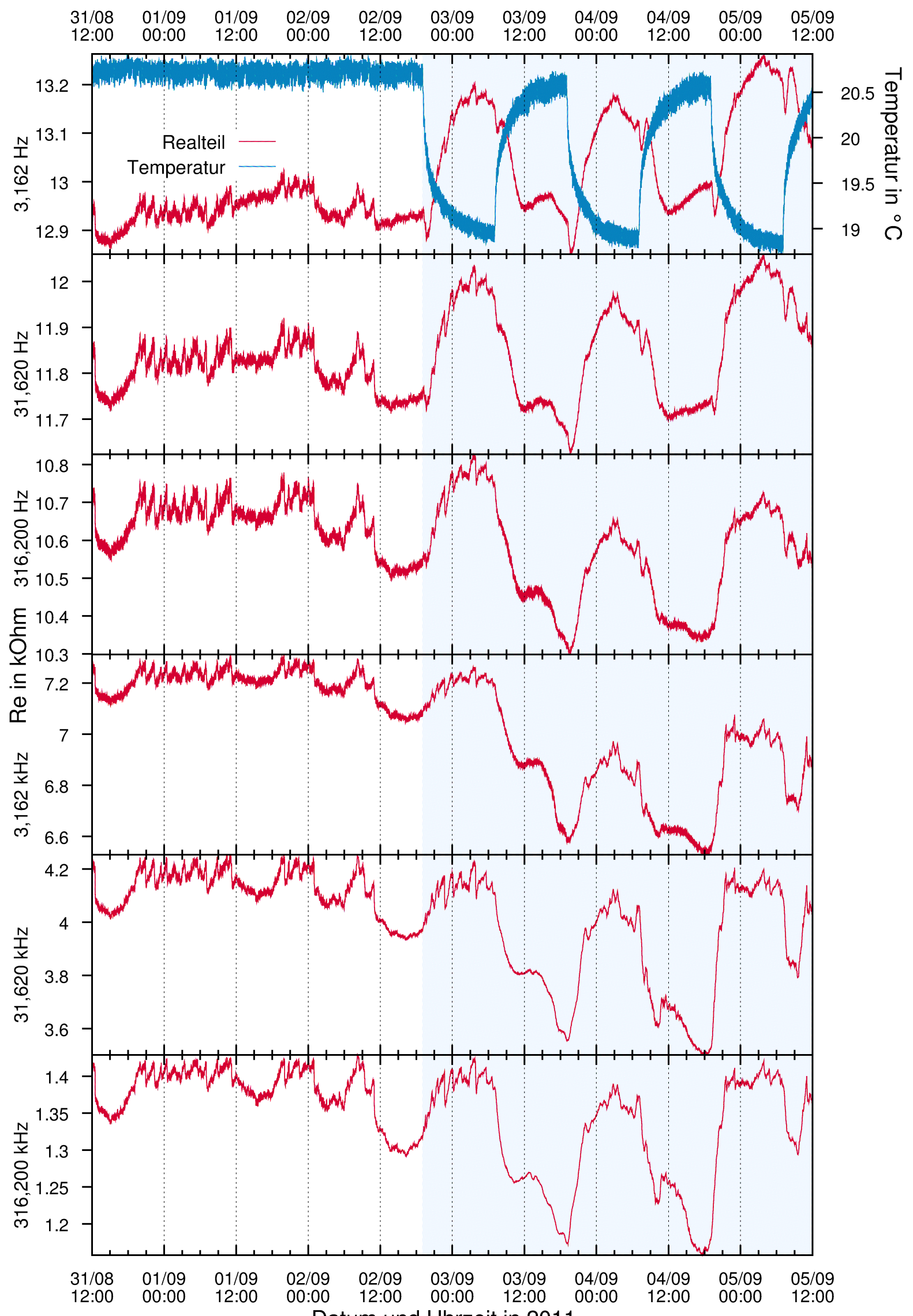

Datum und Uhrzeit in 2011

Abbildung 4.18: Verlauf des Realteils der Impedanz bei sechs verschiedenen Frequenzen vom 31.08.2011 bis 05.09.2011 an einem Weidensteckling in der Klimakammer. In der ersten Teilgrafik (oben) wird zusätzlich die Temperatur dargestellt. 
Vergleicht man die Tief- und Hochpunkte des Realteils der Impedanz bei den verschiedenen Frequenzen während der Phase mit wechselnden Lichtverhältnissen, so ergibt sich hier ein Schwankungsbereich von $2,6 \%-14 \%$.

Auffällig ist, dass bei steigender Frequenz der prozentuale Schwankungsbereich zunimmt. Vergleicht man das Verhältnis zwischen dem Schwankungbereich des Realteils während der Dauerlichtphase und der Schaltphase, so ergeben sich unterschiedliche Werte. Der prozentuale Schwankungsbereich des Realteils während der Hell- und Dunkelphase ist in diesem betrachteten Kollektiv und Zeitraum etwa doppelt so hoch wie der prozentuale Schwankungsbereich während der Dauerlichtphase. Bei einer Schwankung der Temperatur um ca. $2^{\circ} \mathrm{C}$ schwankt der Realteil um ca. 2,4\%-14\% je nach betrachteter Frequenz.

Der Einfluss der Temperatur auf die elektrischen Widerstandswerte (Real- und Imaginärteil der Impedanz) wird weiterhin in Abbildung 4.19 dargestellt. In dieser Darstellung werden nur die Daten aus der Phase mit intervallweise wechselnden Lichtverhältnissen (ab 02.09.2011 um 19:00 Uhr) berücksichtigt. Verglichen werden der Realteil und der Imaginärteil der Impedanz der Nachtphasen mit denen der Tagphasen an fünf Tagen (02.09.2011-06.09.2011) bei sechs verschiedenen Frequenzen. In der Abbildung 4.19a werden die Realteile der Impedanz dargestellt, in Abbildung 4.19b die Imaginärteile der Impedanz. Für die Nachtphase wurden Daten zwischen 03:00 Uhr-06:30 Uhr gewählt, für die Tagphase solche zwischen 15:00 Uhr-18:30 Uhr. Diese gewählten Zeitintervalle aus der Dunkel- bzw. Lichtphase wurden gewählt, da sich in diesen Zeitfenstern die Temperatur eingeregelt hat (vgl. Abbildung 4.18). Der Punkt repräsentiert den Median der Messwerte. In der Box befinden sich $50 \%$ der Werte. Die vertikalen, gestrichelten Linien (whisker) zeigen die weiteren Messwerte außerhalb der Box ohne extreme Ausreißer. Extreme Ausreißer stellen sich folglich als Punkte außerhalb der whisker dar. Ausreißer liegen mehr als das anderthalbfache des Interquartilabstandes unter oder über der Box.

Betrachtet man zunächst den Realteil der Impedanz, so fällt auf, dass sich die Tag- und Nachtwerte bei den niedrigen Frequenzen besonders stark voneinander unterscheiden (z. B. $3,162 \mathrm{~Hz}$ und 31,620 Hz). Die Realteile der Tagwerte sind dabei, wie bereits in Abbildung 4.18 gesehen, deutlich niederohmiger. Weiterhin fällt die größere Streuung der Tagwerte auf. Diese ist bei allen Frequenzen größer als die der Nachtwerte des hier dargestellten Kollektivs. Auch treten hier die meisten Extremwerte auf.

Eine Streuung und das Auftreten von Extremwerten sind ebenso beim Imaginärteil zu beobachten. Beim Vergleich der Imaginärteile bei verschiedenen Frequenzen zwischen Tag und Nacht ist allerdings kein eindeutiges Muster erkennbar. Bei der Frequenz von beispielsweise $3,162 \mathrm{~Hz}$ liegen die Werte in ähnlichen Größenordnungen, wohingegen die Imaginärteile der Tagwerte bei einer Frequenz von $31,620 \mathrm{~Hz}$ höher bzw. bei $3,162 \mathrm{kHz}$ deutlich höher liegen. Ein anderes Bild zeigt sich bei $316,200 \mathrm{kHz}$ und $31,620 \mathrm{kHz}$. Hier liegen die Imaginärteile der Tagwerte unter denen der Nachtwerte. 


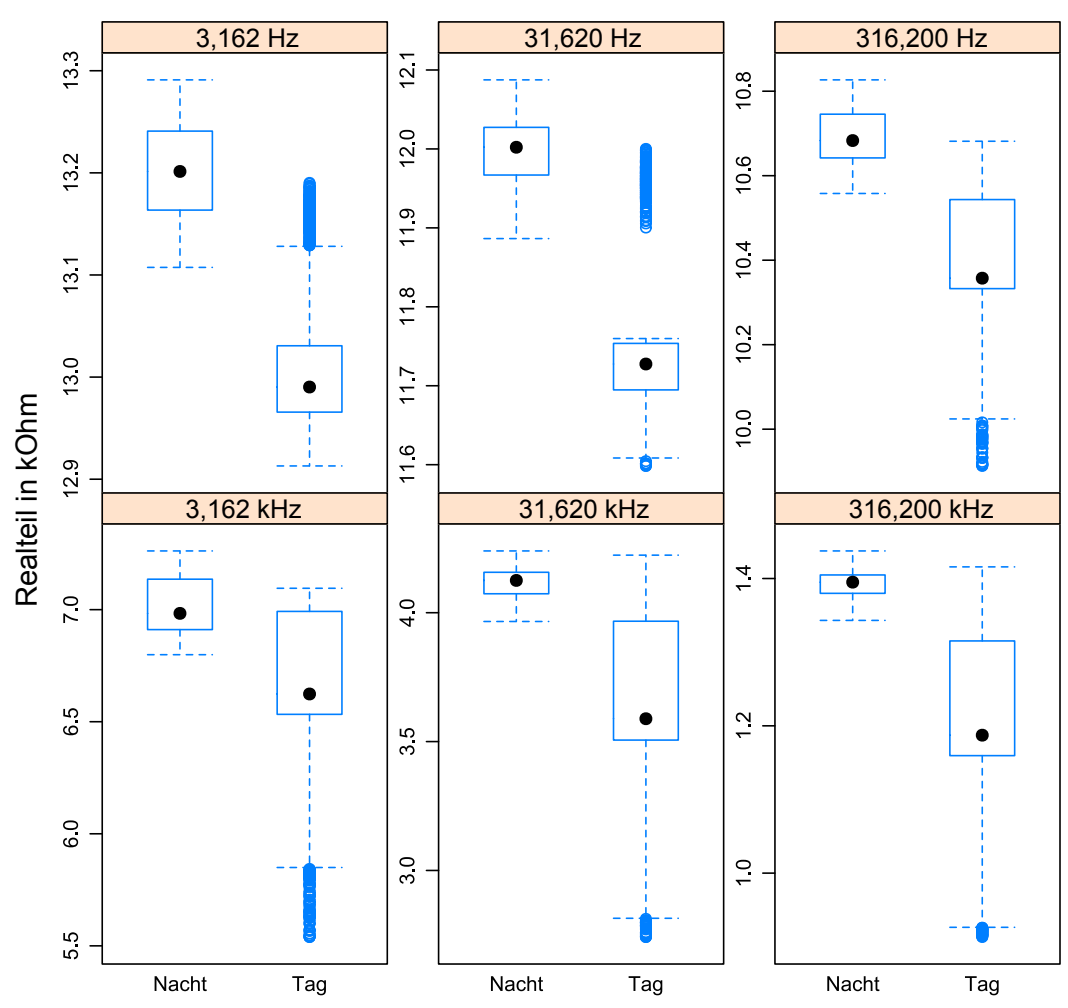

(a)

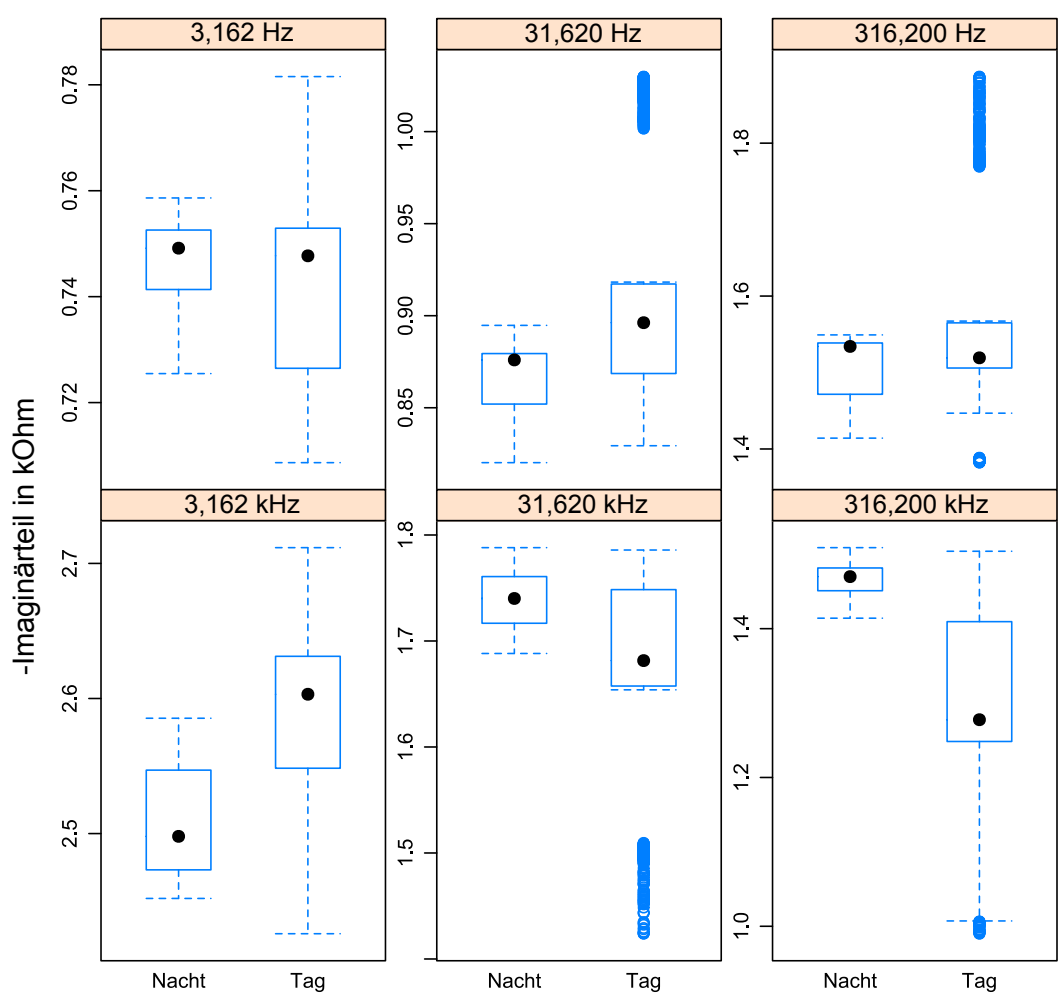

(b)

Abbildung 4.19: Vergleich von Realteil (a) und Imaginärteil (b) der Impedanz anhand von Box-Whisker-Plots bei Tag und Nacht und sechs verschiedenen Frequenzen. 


\section{Einfluss der Dimension der Stecklinge}

In Abbildung 4.20 ist der Zusammenhang zwischen Realteil (Abbildung 4.20a) bzw. Imaginärteil der Impedanz (Abbildung 4.20b) und dem Durchmesser von 16 Weidenstecklingen ( $S a$ lix babylonica) dargestellt. Betrachtet werden acht ausgewählte Frequenzen von ca. $50,1 \mathrm{~Hz}-$ $1 \times 10^{5} \mathrm{~Hz}$.

In Abbildung 4.20a ist bei allen acht betrachteten Frequenzen der deutliche Zusammenhang zwischen dem arithmetischen Mittel des Durchmesser der Stecklinge und der Realteil der Impedanz ersichtlich. Mit zunehmendem Durchmesser sinkt der Realteil der Impedanz. Die Realteile der Impedanz liegen bei den in dieser Testreihe untersuchten Stecklingen zwischen wenigen $\Omega$ bis hin zu $5 \times 10^{4} \Omega$. Die negative Korrelation zwischen beiden Messparametern zeigt einen annähernd linearen Trend. Für die Interpolation der Messwerte wurde eine kubische Splinefunktion mit drei Stützstellen verwendet. Der graue Bereich um die Funktion stellt das $95 \%$ Konfidenzintervall dar. Wie in dieser Abbildung ersichtlich, ist dieser Vertrauensbereich sehr eng.

In Abbildung $4.20 \mathrm{~b}$ ist der Imaginärteil der gemessenen Impedanz über dem Durchmesser aufgetragen. Hier zeigt sich nach Ausgleich mit kubischer Splinefunktion ein positiver Zusammenhang. Dieser ist bei den Frequenzen von $501 \mathrm{~Hz}$ und $100 \mathrm{~Hz}$ gering. Die Ausgleichsfunktion hat eine sehr geringe Steigung. Ab Frequenzen $>501 \mathrm{~Hz}$ wird der Zusammenhang deutlich größer. Bei ansteigendem gemitteltem Durchmesser steigt ebenfalls der Imaginärteil mit einem annähernd linarem Trend. Der Imaginärteil reicht hier in den negativen Bereich bis ca. $20 \mathrm{k} \Omega$. Auffällig ist, dass bei niedriger Frequenz der Imaginärteil der Impedanz sehr geringe Phasenwinkel aufweist. Je nach Steckling treten bis ca. $50 \mathrm{~Hz}$ darüber hinaus positive Phasenwinkel auf. Der Imaginärteil insgesamt erscheint daher ebenfalls teils positiv. Dieser Befund ist bereits in Abschnitt 4.3.1 angesprochen. 


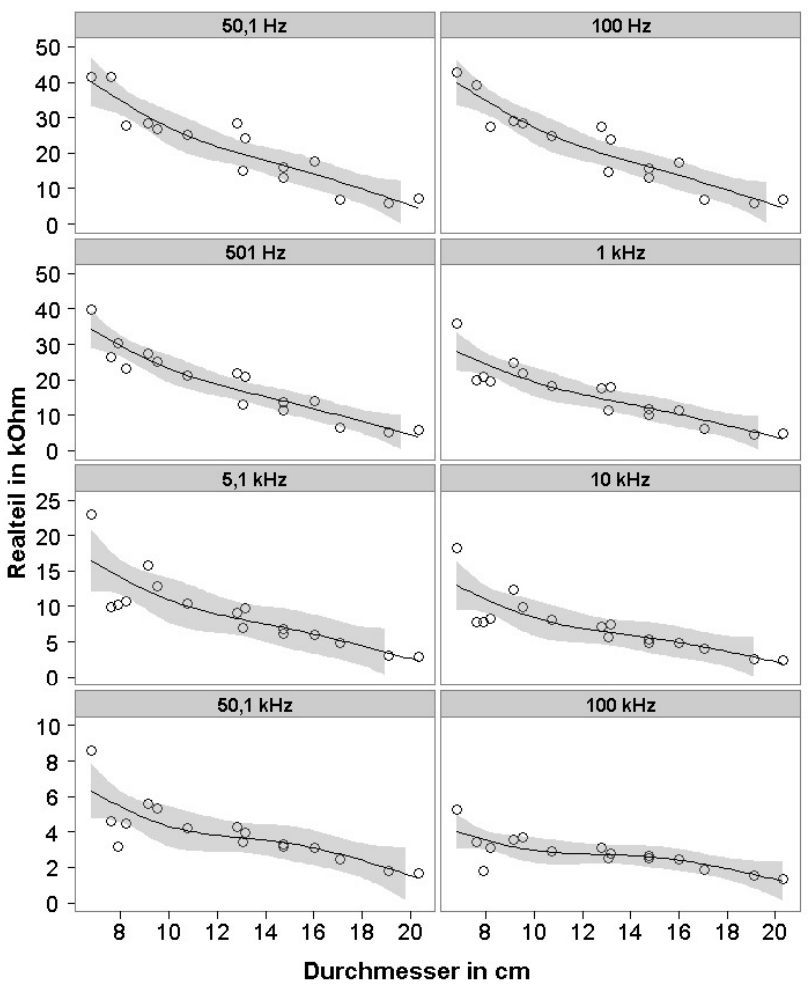

(a)

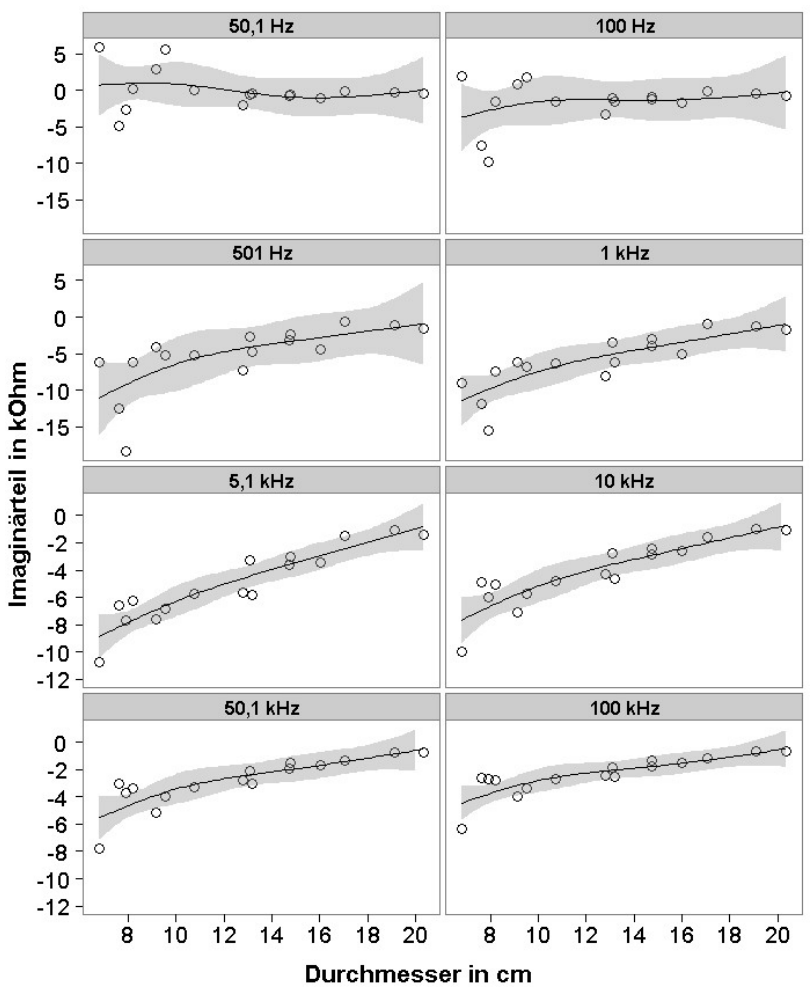

(b)

Abbildung 4.20: Zusammenhang zwischen Durchmesser und Impedanz bei acht verschiedenen Frequenzen. (a) Realteil über Durchmesser, (b) Imaginärteil über Durchmesser. Weitere Erläuterungen zu dieser Abbildung finden sich im Text. 


\section{Ergebnisse}

\section{Einfluss der Leitgewebe der Stecklinge}

Nachdem diese Einflussgröße in Abschnitt 4.1 .3 bereits monofrequent untersucht wurde (Erdungsmessung), wird dieser Zusammenhang hier multifrequent betrachtet.

In Abbildung 4.21 werden die in Abschnitt 4.3 .2 ausgewählten zwei Stecklinge berücksichtigt. Bei den beiden Stecklingen wurde jeweils der Real- und der Imaginärteil der Impedanz, ermittelt in zwei verschiedenen Messebenen $\left(\mathrm{M}_{1}\right.$ und $\mathrm{M}_{2}$; vgl. Abbildung 3.8, über der Frequenz dargestellt (vgl. dazu Abschnitt 3.3.4). Die Auswertung der restlichen 13 Stecklinge ist im Anhang enthalten (Abbildungen A.6 bis A.9.

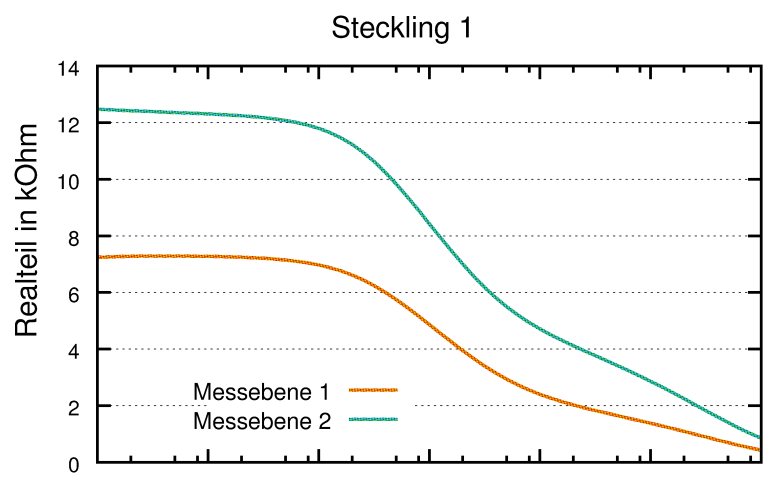

Steckling 2
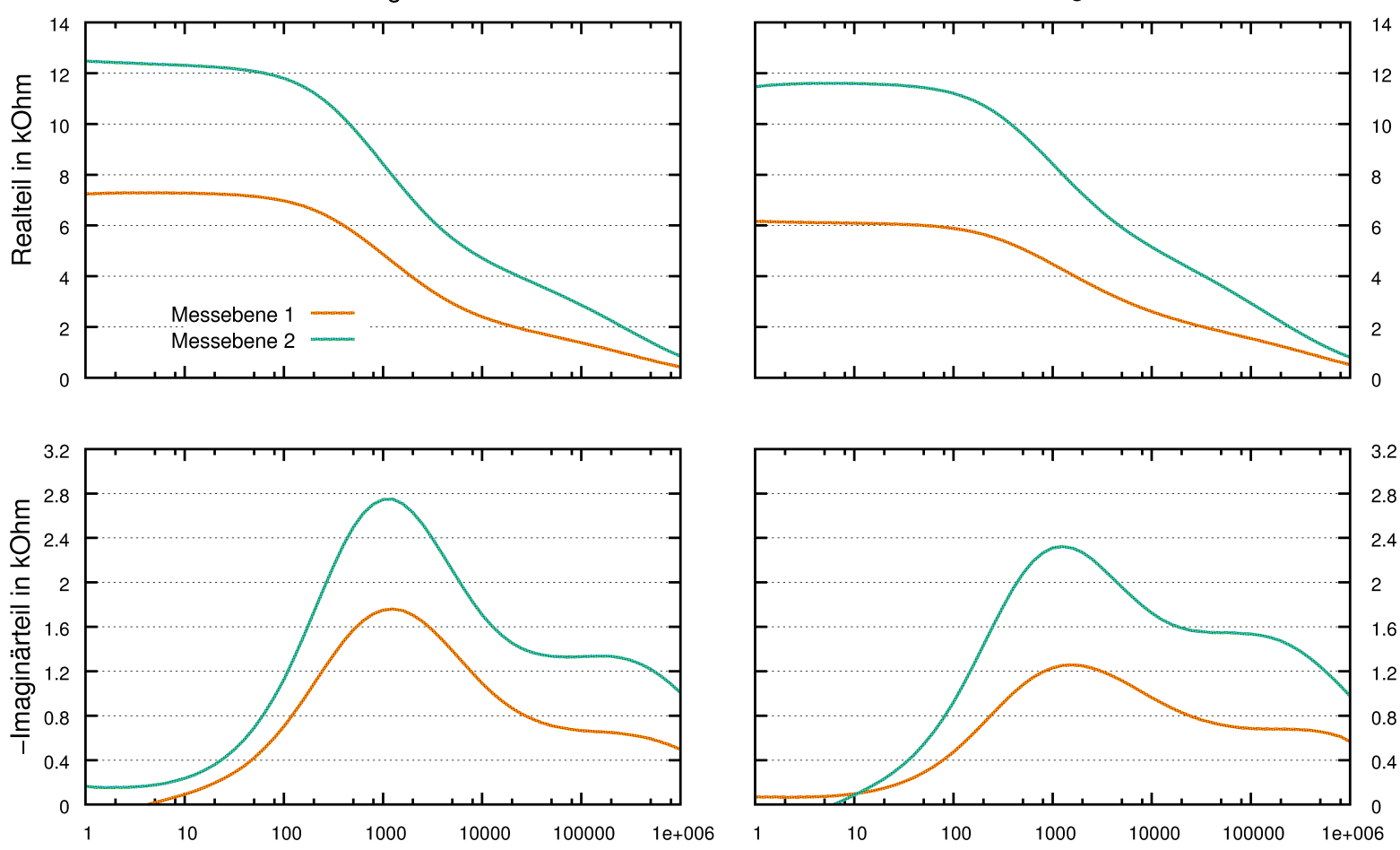

$\log$ Frequenz in $\mathrm{Hz}$

Abbildung 4.21: Vergleich des Realteils (oben) und des Imaginärteils (unten) über der Frequenz in zwei verschiedenen Messebenen (Messebene 1 und 2) zweier Stecklinge (links: Steckling 1, rechts: Steckling 2).

Betrachtet man zunächst den Realteil der Impedanz auf beiden Messebenen über der Frequenz, so fällt auf, dass der Realteil bei zunehmender Frequenz kontinuierlich geringer wird. Dieser Befund lässt sich sowohl für Messebene 1, als auch für Messebene 2 erkennen, so dass die Graphen der beiden Messebenen über weite Teile des Spektrums parallel verlaufen.

Beim Imaginärteil fällt auf, dass dieser über der Frequenz betrachtet zunächst ansteigt, bei $1 \mathrm{kHz}$ sein Maximum erreicht, um anschließend wieder zu sinken. Diese Zusammenhänge gelten relativ betrachtet für beide Messebenen. Absolut betrachtet wird deutlich, dass sowohl der Real-, als auch der Imaginärteil der Messebene 1 fast durchgängig kleiner ist als die entsprechenden Werte der Messebene 2. Eine Ausnahme dabei stellt Steckling 2 bei $10 \mathrm{~Hz}$ da. Hier ist der Imaginärteil der Impedanz bei Messebene 1 größer als der der Messebene 2. Ähnliche 
Abweichungen finden sich auch bei den Stecklingen 6, 8, 9, 13 und 15. Betrachtet man die Realteile der beiden Messebenen bei der hier visualisierten Auswahl relativ zueinander, so fällt auf, dass die Messwerte ermittelt aus der Messebene 2 teils doppelt so hoch sind wie aus der Messebene 1.

Bildet man die Differenz der Spektren, die in Abschnitt 3.3.3 dargestellt wird, so ergibt sich der Anteil, der dem Leitgewebe des Stecklings mit einer Länge von $50 \mathrm{~mm}$ entspricht. Es ergeben sich hier prozentuale Werte von 35\%-60\%. $50 \mathrm{~mm}$ verholztes Stecklingsgewebe machen in dieser Anordnung somit ca. ein Drittel bis mehr als die Hälfte des Real-, wie Imaginärteils der Impedanz aus.

\subsubsection{Hauptversuche}

\section{Allgemeine Betrachtung der physikalischen und biologischen Daten}

Abbildung 4.22 zeigt das Wurzelwachstum beispielhaft an Steckling 9 aus Hauptversuch 1 im Zeitraum vom 05.02.2011 bis 23.02.2011 anhand von sieben Fotos in der Klimakammer.

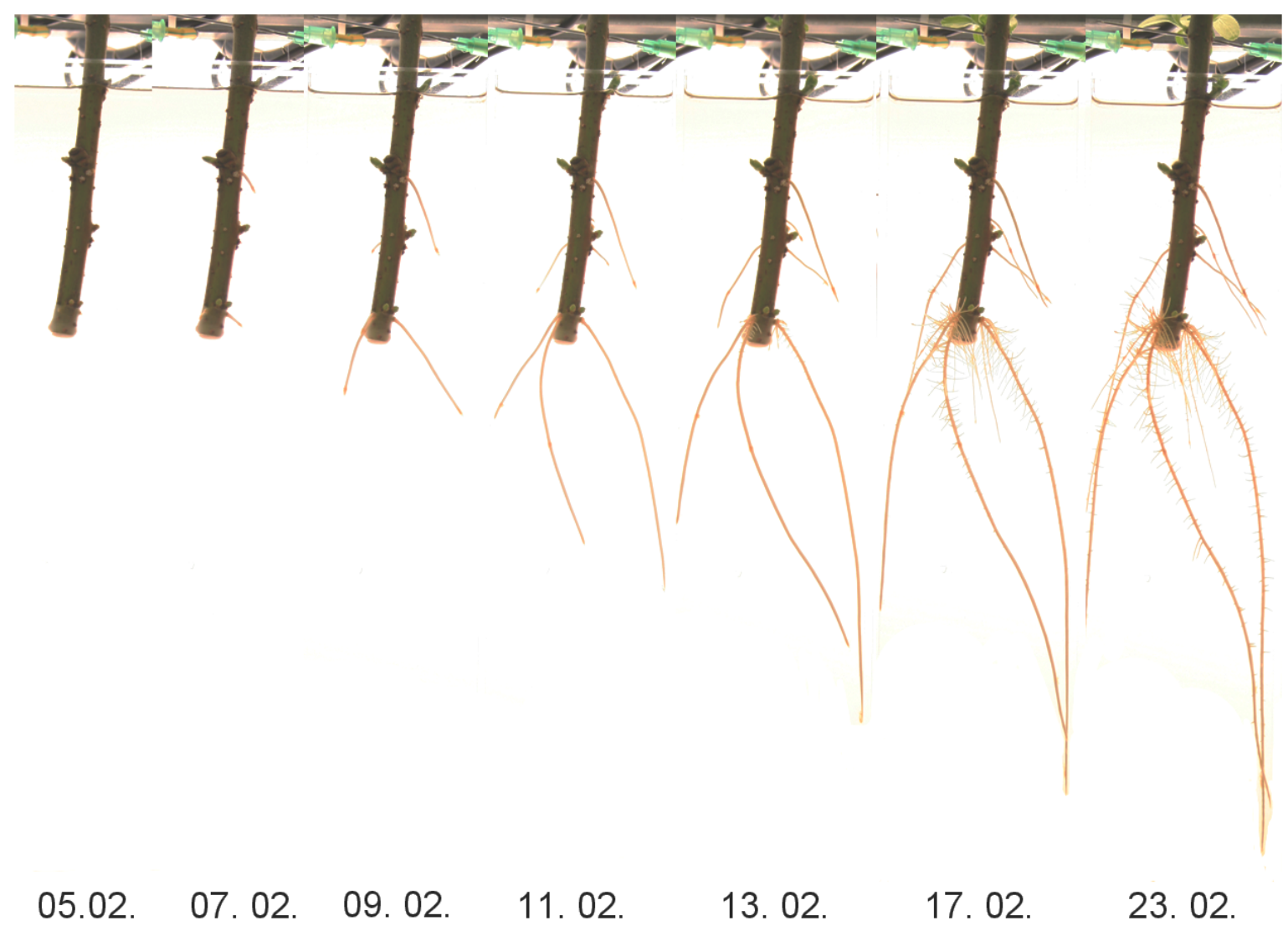

Abbildung 4.22: Verlauf des Wurzelwachstums von Steckling 9 im Zeitraum vom 05.02.2011 23.02.2011 in der Klimakammer anhand von sieben Fotos. 
Der hier exemplarisch dargestellte Steckling zeigt ein typisches Wachstum des untersuchten Kollektivs. Dieses Wachstum über 18 Tage wird bereits visuell deutlich. Typischerweise zeigen sich während des Wurzelwachstums verschiedene Wachstumsphasen. Zunächst werden lediglich Wurzeln erster Ordnung ausgebildet. Diese erste Wachstumsphase beginnt bei den hier untersuchten Stecklingen zu unterschiedlichen Zeiten. Wurzeln zweiter Ordnung treten in der zweiten Wachstumsphase auf, beispielsweise bei dem hier exemplarisch abgebildeten Steckling ab dem 13.02. Das Entfalten von Blattorganen beginnt erst deutlich nach Ausbildung der Wurzeln erster Ordnung (hier ab ca. 14.02.).

Die Darstellung des Wurzelwachstums während des Versuchs ist durch die beschriebene Auswertung der Fotos mit dem Programm WinRHIZO® auch quantitativ möglich. In Abbildung 4.23 werden zwei wesentliche Größen über der Zeit (Anzahl der Tage), in Abbildung 4.23a ist die Wurzellänge über der Zeit, in Abbildung 4.23b die Wurzeloberfläche über der Zeit dargestellt.

Als Nullpunkt der Abszisse wurde der 04.02.2011 ausgewählt, da an diesem Tag bei den ersten drei der 15 untersuchten Stecklinge erstmalig Wurzeln in Erscheinung treten (Steckling 2, 3 und 9). Andere Stecklinge (Steckling 1, 4, 5, 6, 7, 10, 11, 12, 13, 14) bilden erst später bzw. deutlich später (Steckling 15) Wurzeln aus. Steckling 8 ist bei der Betrachtung von Wurzellänge und -oberfläche nicht aufgeführt, da dieser weder Wurzeln, noch Blattorgane ausgebildet hat.

Betrachtet man alle 14 dargestellten Stecklinge, so fällt auf, dass mit Hilfe des Auswerteprogramms das Wurzelwachstum gut abgebildet werden kann. In nahezu allen Fällen ist eine kontinuierliche Zunahme zu erkennen. Ausnahmen bilden dabei beispielsweise Steckling 2, 4 und 5. Hier zeigt sich teilweise ein scheinbarer Rückgang von Wurzelmasse resp. Wurzeloberfläche, der aber durch die Ausgleichsfunktion gut interpoliert werden kann. Die Steigung der Ausgleichskurve ist in nahezu allen Fällen zunächst steil und flacht nach einiger Zeit leicht ab. Im Bereich der geringen Steigung treten die angesprochenen Probleme des scheinbaren Rückgangs der Wurzellänge (Wurzeloberfläche) auf. Die maximalen Gesamtlängen der Wurzeln eines jeweiligen Stecklings liegen am Ende des Untersuchungszeitraumes zwischen ca. $6 \mathrm{~cm}$ und $170 \mathrm{~cm}$. Die maximalen Gesamtoberflächen der Wurzeln liegen zwischen ca. $1 \mathrm{~cm}^{2}$ und $28 \mathrm{~cm}^{2}$.

In Abbildung 4.24 ist weiterhin der Zusammenhang zwischen diesen beiden biologischen Größen erkennbar. Dargestellt wird jeweils die Wurzellänge auf der Abszisse gegen die Wurzeloberfläche auf der Ordinate der 14 untersuchten Stecklinge. In den Einzeldiagrammen befinden sich darüber hinaus die Funktionen der Ausgleichsgeraden mit den jeweiligen Bestimmtheitsmaßen $\left(r^{2}\right)$ für die Anpassung mit einem robusten linearen Modell. Daraus ist ersichtlich, dass Wurzellänge und Wurzeloberfläche sehr eng miteinander korrelieren. Lediglich im Bereich der geringen Wurzellängen- und oberflächen scheint der Zusammenhang einer Funktion mit größerer Steigung zu folgen. Hier liegen die Abweichungen der Messwerte zum Modell höher als in Bereichen mit größerer Wurzellänge- und oberfläche. Besonders deutlich ist dieser Befund bei Steckling 2, 5 und 6. Dennoch liegen die Bestimmtheitsmaße zwischen 0,41 und 0,81 bei Untersuchung der Daten mit diesem Modell. 


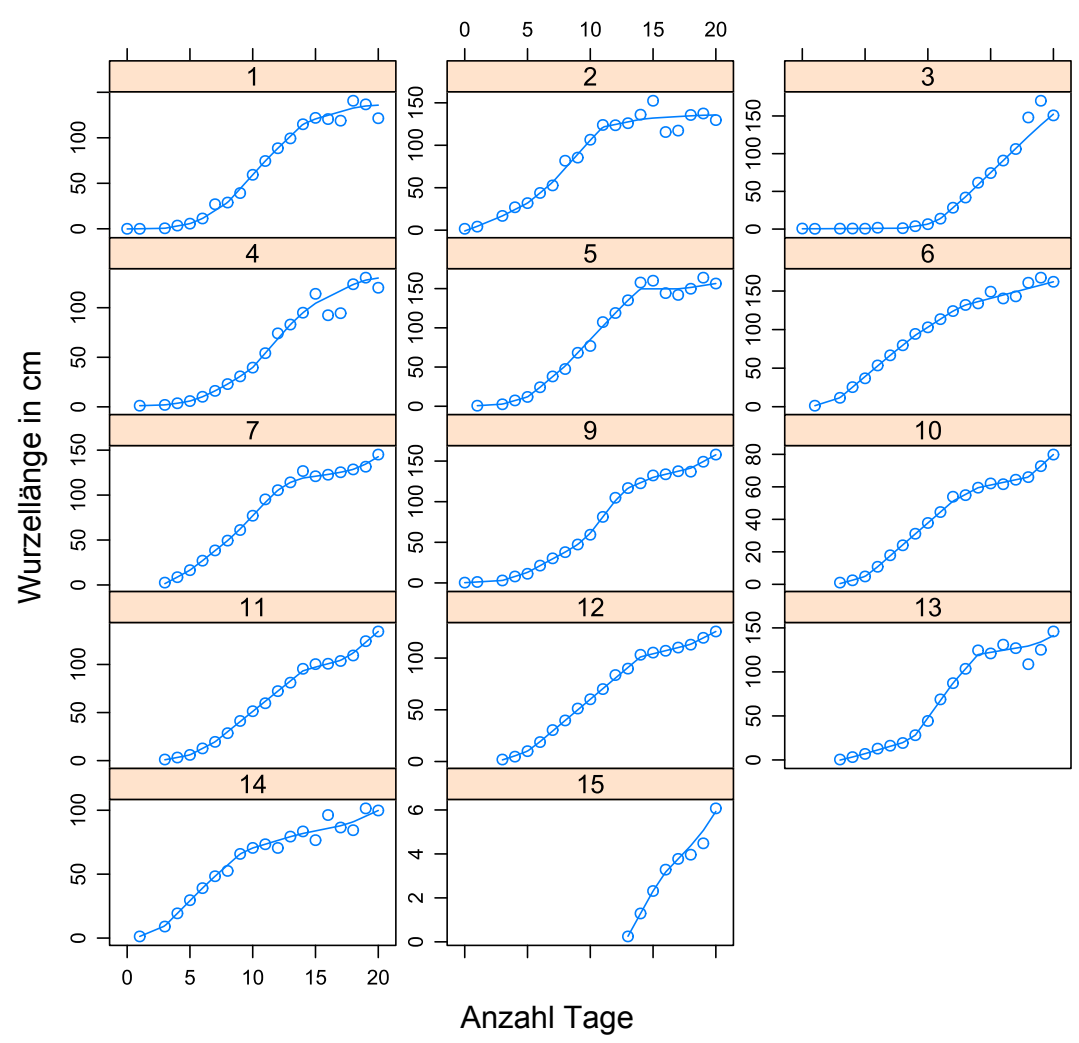

(a)

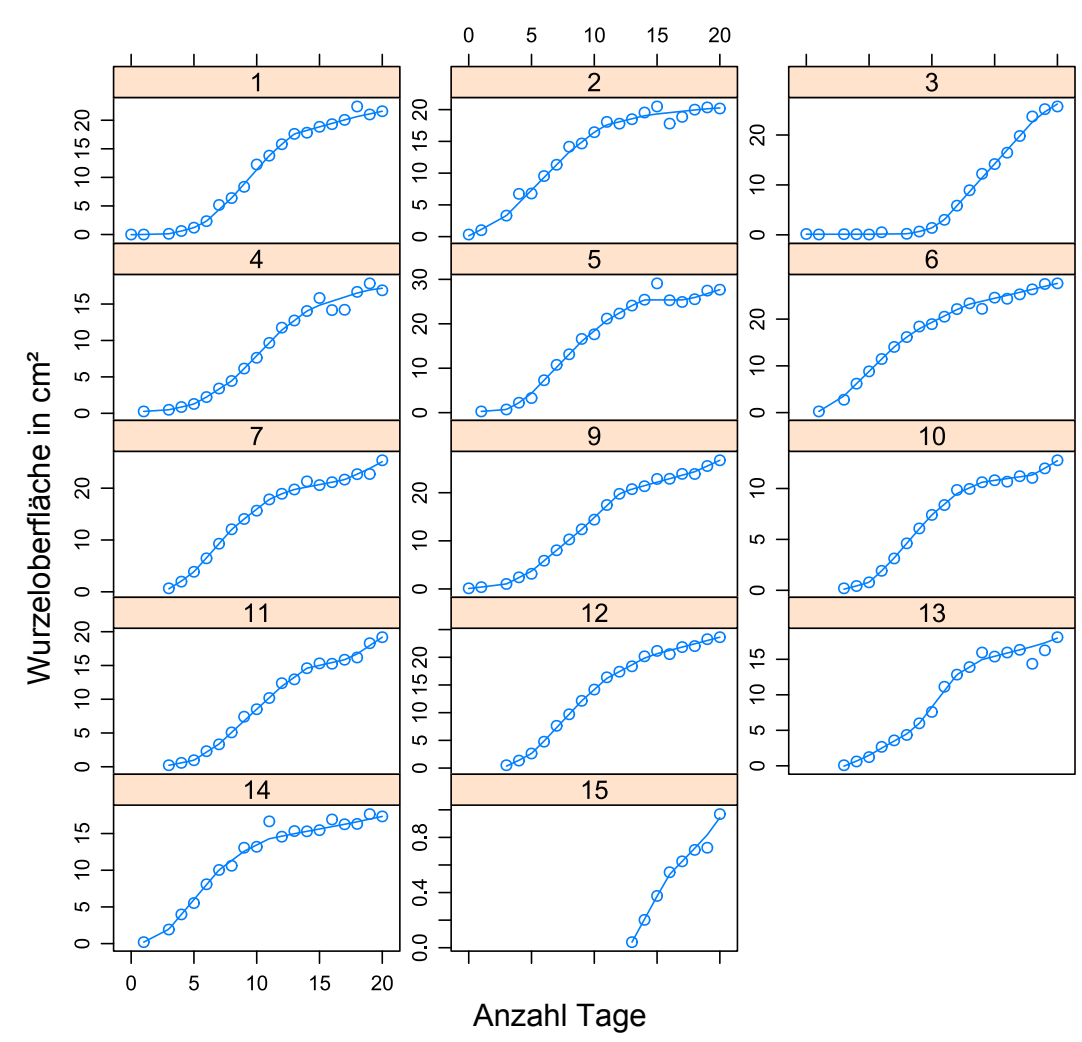

(b)

Abbildung 4.23: Auswertung der Daten aus WinRHIZO® für 14 Weidenstecklinge aus Hauptversuch 1 (a) Wurzellänge und (b) Wurzeloberfläche. 


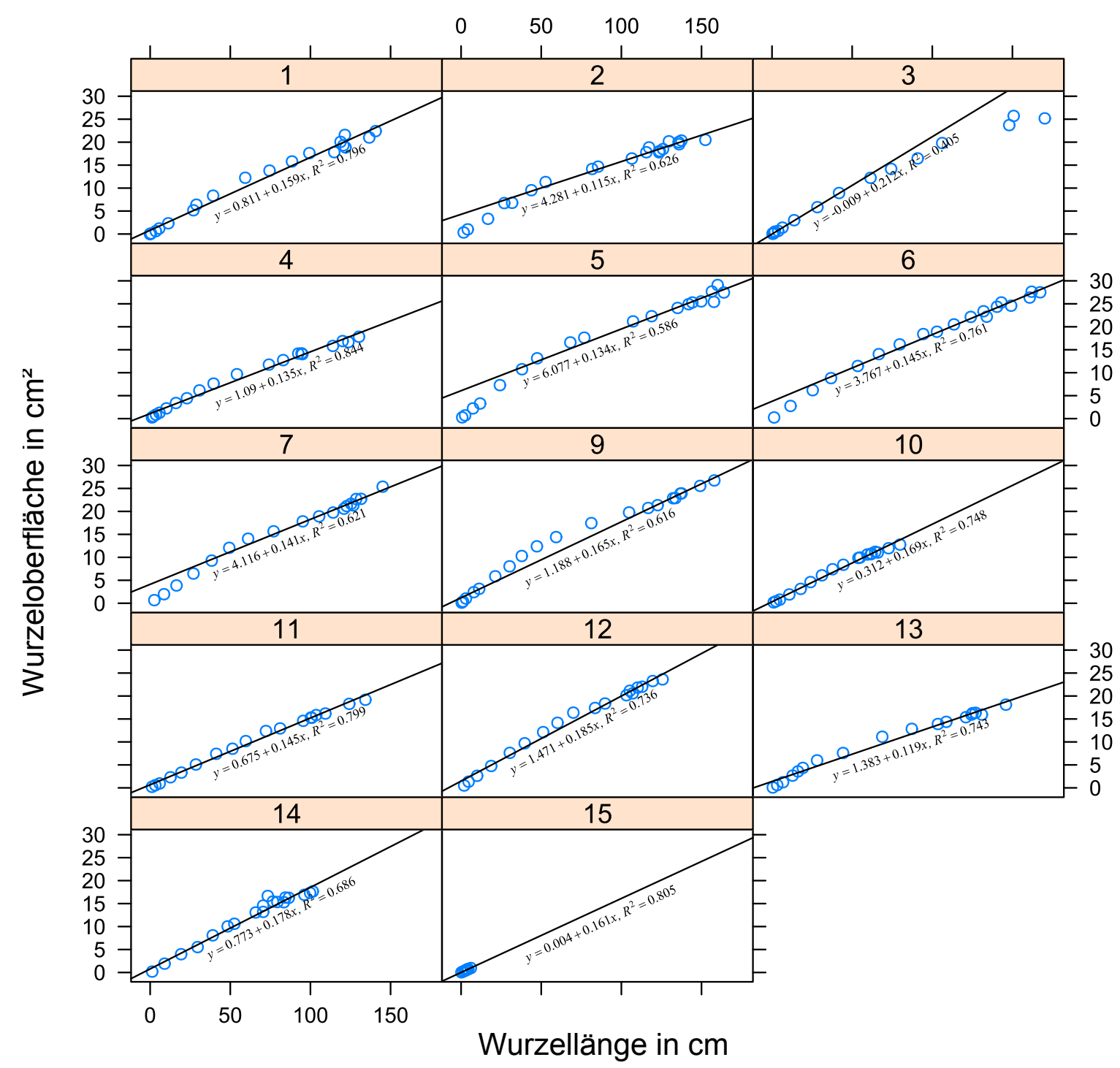

Abbildung 4.24: Zusammenhang zwischen Wurzellänge und Wurzeloberfläche der Stecklinge aus Hauptversuch 1.

Das Wurzelwachstum der Stecklinge in Hauptversuch 2 unterscheidet sich von dem aus Hauptversuch 1 deutlich. Die dickeren Stecklinge des zweiten Versuches bilden bedeutend später erste Wurzelspitzen aus. Außerdem treten im gesamten Versuchszeitraum lediglich Wurzeln erster Ordnung hervor. Quantitativ betrachtet fallen zudem die geringeren Wurzellängen und Wurzeloberflächen auf. So entwickeln sich in diesem Versuch insgesamt maximale Wurzellängen von ca. $44 \mathrm{~cm}$ und Wurzeloberflächen von $9 \mathrm{~cm}^{2}$. Blattorgane werden in dem betrachteten Kollektiv und Zeitraum sehr gering ausgebildet. Trotzdem kann das verzögerte und geringere Wurzelwachstum durch das angesprochene Programm ebenfalls gut abgebildet werden. Wie in Abbildung 4.25 erkennbar, zeigt sich in diesem Versuch, dass alle untersuchten Stecklinge in unterschiedlicher Dimension Wurzeln gebildet haben. 


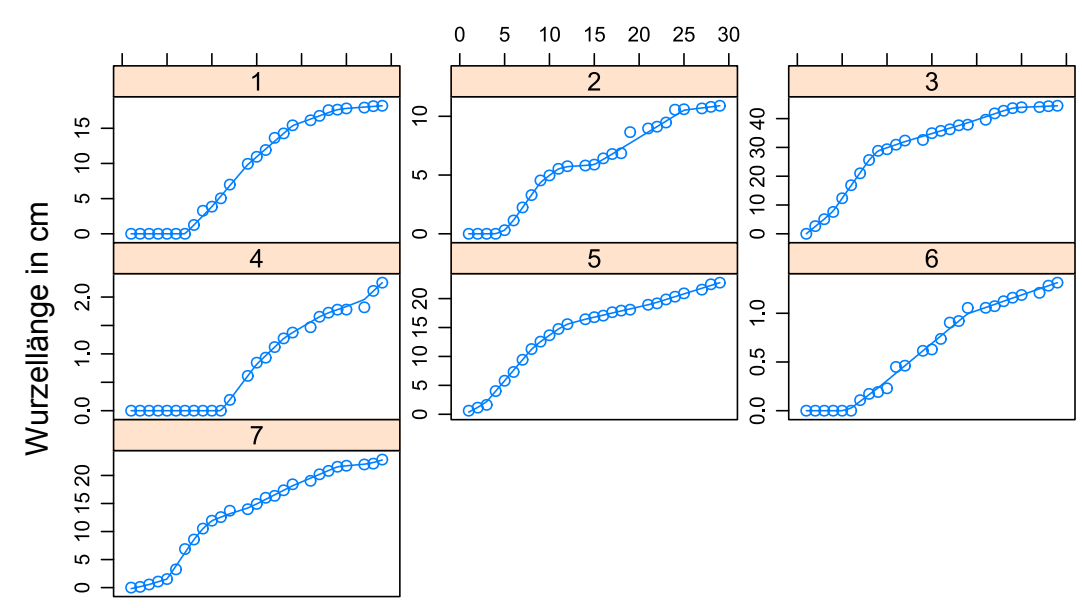

Anzahl Tage

(a)

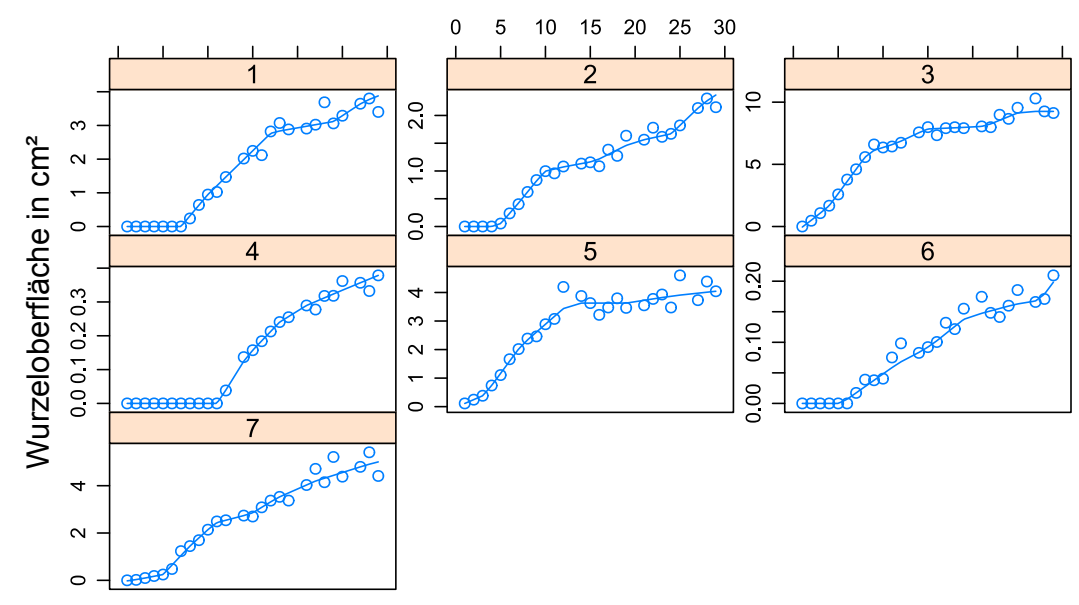

Anzahl Tage

(b)

Abbildung 4.25: Auswertung der Daten aus WinRHIZO® für 7 Weidenstecklinge aus Hauptversuch 2 (a) Wurzellänge und (b) Wurzeloberfläche.

Steckling 3 weist hier die maximalen Werte auf, wohingegen Steckling 6 zuletzt austreibt und am Ende des Versuchszeitraums die kleinsten Werte aufweist. Der Verlauf des Wurzelwachstums hat, anders als im Hauptversuch 1, einen annähernd linearen Trend.

Nachfolgend sind die Ergebnisse der impedanzspektroskopischen Untersuchung von Hauptversuch 1 dargestellt. Dafür wurde exemplarisch der bereits fotografisch vorgestellte Steckling 9 dieses Versuches genauer untersucht.

In Abbildung 4.26 sind Realteil und Imaginärteil der Impedanz über der Zeit dargestellt. Auf der Ordinate ist der Ausschnitt (29.01.2011-26.02.2011) des Versuchszeitraums visualisiert. Der Realteil ist in diesem Diagramm der 1. Ordinate, der Imaginärteil der 2. Ordinate zugeordnet. 


\section{Ergebnisse}

Betrachtet werden sechs verschiedene Frequenzen von 3,162 Hz - 316,200 kHz. Beim Vergleich der Einzelgrafiken untereinander soll auf die unterschiedliche Skalierung der jeweiligen Ordinaten hingewiesen werden.

Bei Betrachtung aller sechs Graphen, fällt die Periodizität innerhalb des Versuchszeitraumes wegen der Licht- und Dunkelphasen in der Klimakammer auf (Tagesgänge).

Sieht man sich die tiefsten vier Frequenzen dieser Auswahl an, so erkennt man ein rasches Absinken des Realteils vom Beginn des betrachteten Zeitraumes bis ca. 05.02.2011 (1. farblich hinterlegte Fläche). Diese „Einlaufphase“ zeigt sich in abgeschwächter Deutlichkeit auch beim Imaginärteil. Besonders stark ist dieser Befund bei 31,620 Hz. An dieser Stelle sei darauf verwiesen, dass der Versuch bereits am 27.01.2011 um ca. 20:00 Uhr gestartet wurde. Bis zum Startpunkt der hier abgebildeten Grafik liegen allerdings keine verlässlichen Daten vor, so dass auf der Abszisse der Startpunkt mit dem 29.01.2011 festgesetzt wurde. Es kann davon ausgegangen werden, dass die beschriebene Einlaufphase noch weiter im hochohmigen Bereich beginnt.

Das eigentliche Wurzelwachstum beginnt bei diesem Steckling ab dem 07.02.2011 im 2. farblich hinterlegten Bereich (siehe dazu auch Abbildung 4.22 sowie Abbildung 4.23). Ab diesem Zeitpunkt ist die Entwicklung der beiden betrachteten Größen in eine bestimmte Richtung deutlich geringer. Bei allen Frequenzen ist lediglich ein leichter Trend zu erkennen, der durch die feinere Skalierung der Ordinaten im höherfrequenten Bereich besonders deutlich wird. Bis zum 15.02.2011 sinkt zunächst der Realteil ab, während er nach diesem Zeitpunkt allmählich ansteigt. Der Trend des Imaginärteils ist weniger deutlich. Beachtet werden muss an dieser Stelle, dass im niederfrequenten Bereich $(3,162 \mathrm{~Hz})$, wie bereits angesprochen, positive Werte gemessen wurden (positive Phasenwinkel). Im hochfrequenten Bereich $(316,200 \mathrm{kHz}$ ) ist die Skalierung der Ordinaten sehr eng, so dass hier Ausreißer und periodische Schwankungen besonders gut zum Ausdruck kommen.

In Abbildung 4.27 sind einige der gemessenen sweeps dieses Stecklings in der komplexen Ebene dargestellt. Verglichen werden hier vier sweeps zu vier verschiedenen Zeitpunkten. Neben Messwerten des unbewurzelten Stecklings 9 aus der bereits erwähnten Einlaufphase vom 29.01.2011, werden die Messwerte dieses Stecklings vom 05.02.2011, nach Ende dieser Einlaufphase, abgebildet (ohne Wurzeln). Des Weiteren finden zwei weitere Graphen Berücksichtigung. Zum einen sind dies Messwerte, bei denen Wurzeln erster Ordnung (12.02.2011) am Steckling ausgebildet waren, zum anderen Messwerte, bei denen Wurzeln zweiter Ordnung am Steckling in Erscheinung traten (23.02.2011). Alle vier sweeps wurden zur gleichen Tageszeit ausgewählt (ca. 13:30 Uhr).

Erkennbar ist insgesamt, dass die Graphen einen ähnlichen Verlauf aufweisen. Die Messwerte der Einlaufphase unterscheiden sich quantitativ betrachtet deutlich von den Werten der übrigen Tage. Lediglich im hochfrequenten Bereich (linker Bildbereich) laufen die Graphen zusammen. Im direkten Vergleich der Messwerte von der Einlaufphase zu den Messwerten des unbewurzelten Stecklings, ist der Unterschied besonders groß. Im niederfrequenten Bereich ist der Unterschied am größten (rechter Bildbereich). Bemerkenswert scheint außerdem der Vergleich der beiden Graphen des unbewurzelten und des gleichen nach einer Woche später 


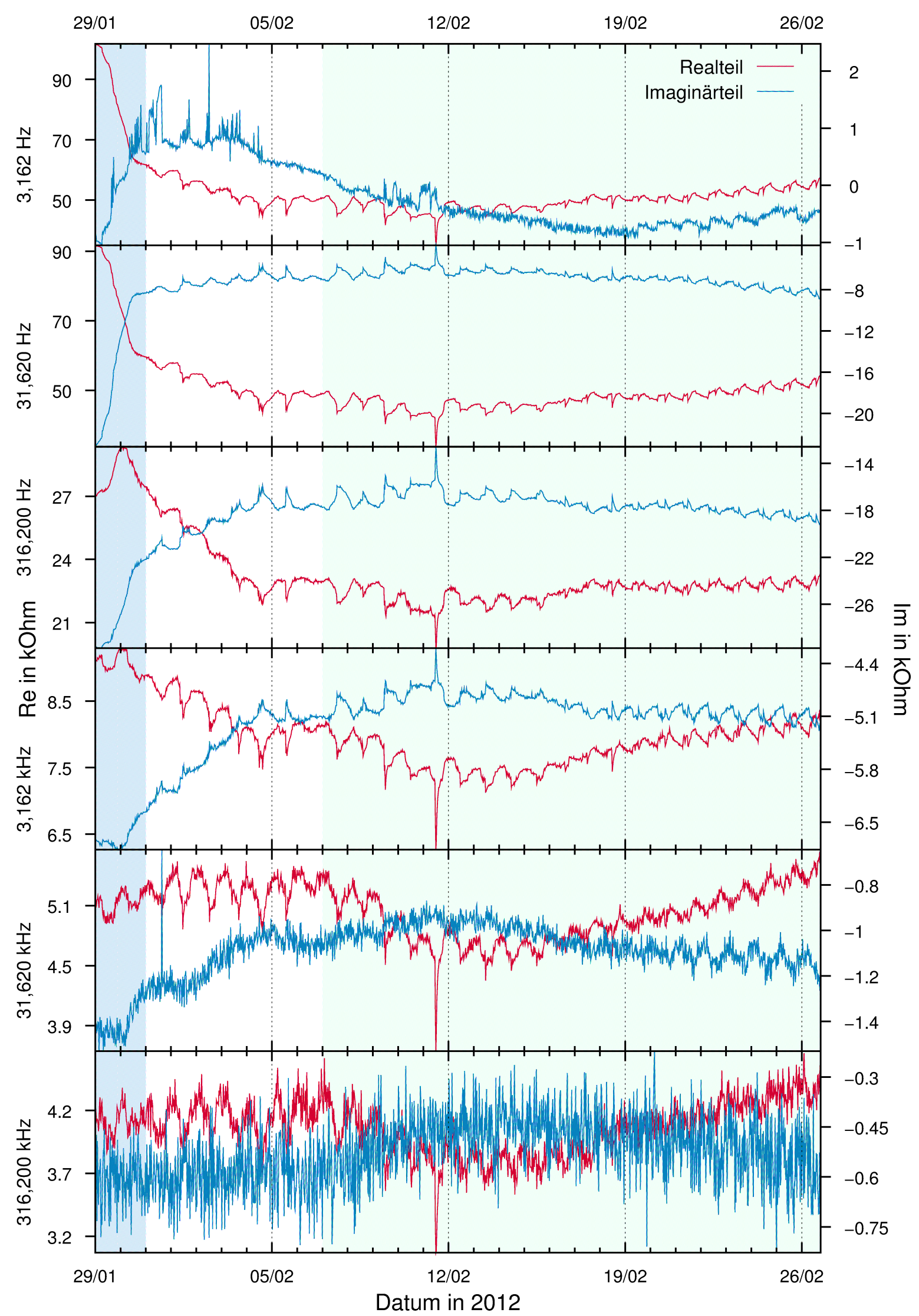

Abbildung 4.26: Verlauf von Real- und Imaginärteil von Steckling 9 bei sechs verschiedenen Frequenzen über der Zeit von Hauptversuch 1. 


\section{Ergebnisse}

gemessenen bewurzelten Stecklings. Obwohl hier deutliche Wurzeln (Wurzeln erster Ordnung) gebildet wurden, ist kaum ein Effekt im Impedanzspektrum zu erkennen. Da Imaginär- und Realteil im weiteren Verlauf leicht ansteigen (siehe auch Abbildung 4.26), macht sich dieser Effekt auch in der komplexen Ebene, besonders im niederfrequenten Bereich bemerkbar.

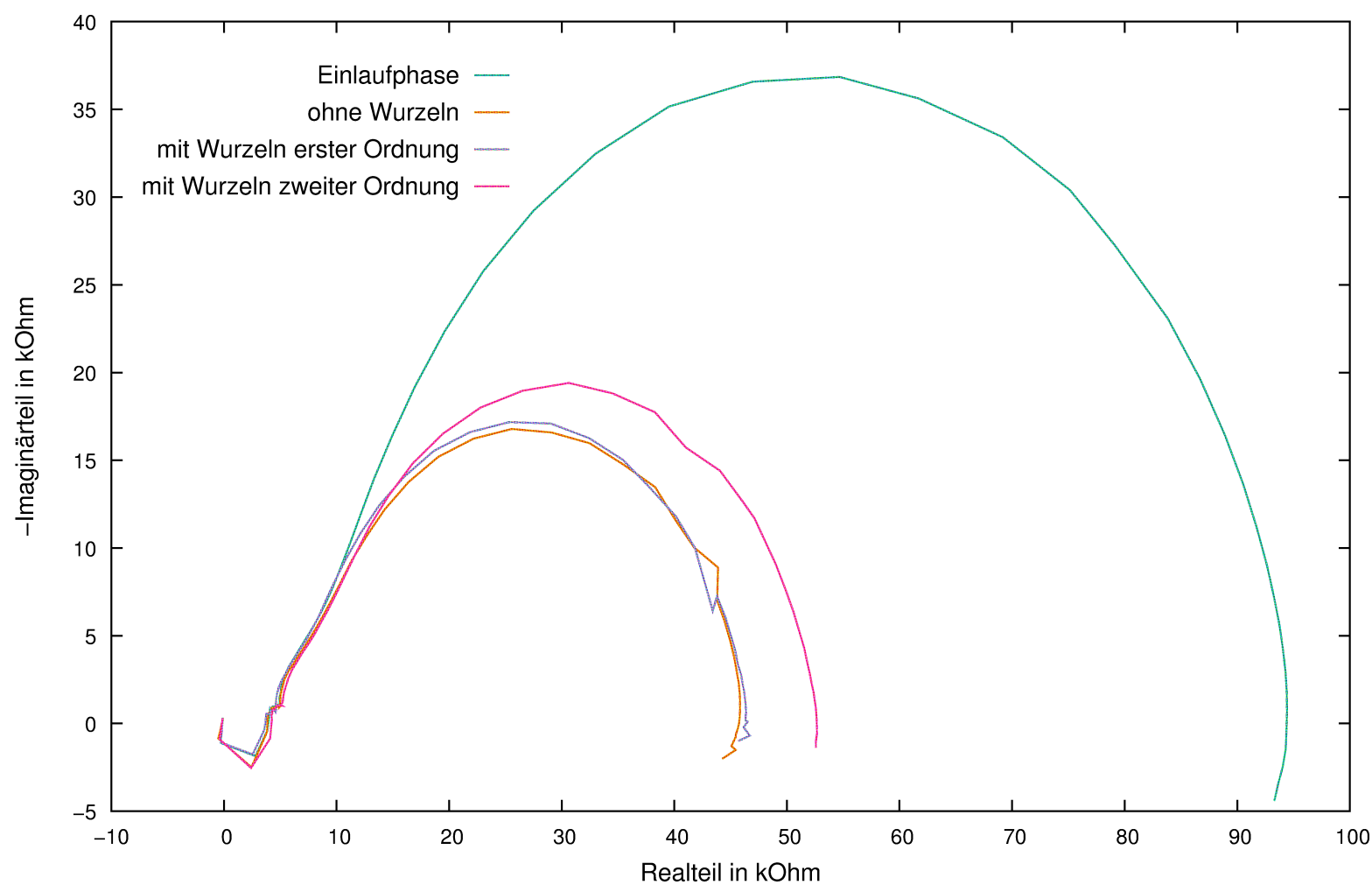

Abbildung 4.27: Ausgewählte Messergebnisse aus Hauptversuch 1 von Steckling 9 in der komplexen Ebene.

Abbildung 4.28 zeigt den Zusammenhang zwischen Realteil der Impedanz und der Wurzellänge (Abbildung 4.28a) bzw. dem Imaginärteil der Impedanz und der Wurzellänge (Abbildung 4.28b bei sechs Frequenzen exemplarisch anhand von Steckling 9. Für diesen Zweck wurden sechs verschiedene Frequenzen aus sweeps von 21 Tagen um ca. 13:30 Uhr mit der an diesem Tag ermittelten Wurzellänge korreliert. In den Grafiken wurden die Messwerte ab dem Zeitpunkt dargestellt, bei dem erstmalig Wurzeln in Erscheinung traten. Die Anpassung der Messwerte erfolgte dabei durch eine Quantilregression als Splinefunktion. Der farblich hinterlegte Bereich zeigt den $95 \%$ Vertrauensbereich.

Sieht man sich zunächst den Realteil der Impedanz (Abbildung 4.28a) an, so fällt auf, dass bei allen sechs Frequenzen die Ausgleichsfunktionen anfänglich eine negative Steigung haben. Bei einer Wurzellänge von ca. $60 \mathrm{~mm}$ ändert sich dieser Befund und der Zusammenhang zwischen Realteil und Wurzellänge wird positiv mit z. T. sehr starken Steigungen (z. B. bei einer Frequenz von $3160 \mathrm{~Hz}$ ). Auffällig ist außerdem, dass der Vertrauensbereich im rechten Teil der Einzelgrafiken deutlich enger wird. Dieser Befund ist besonders bei einer Frequenz von $31600 \mathrm{~Hz}$ ausgeprägt. 


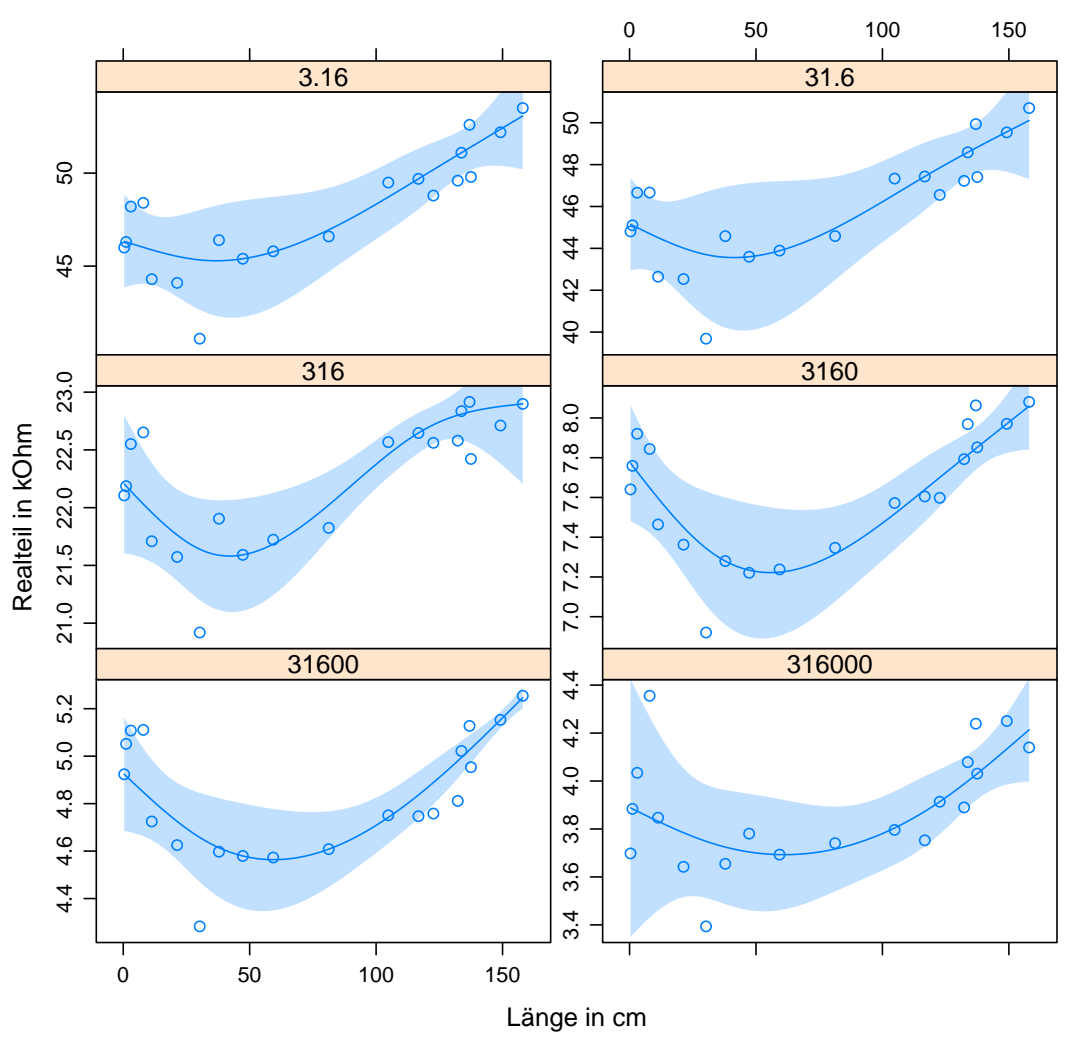

(a)

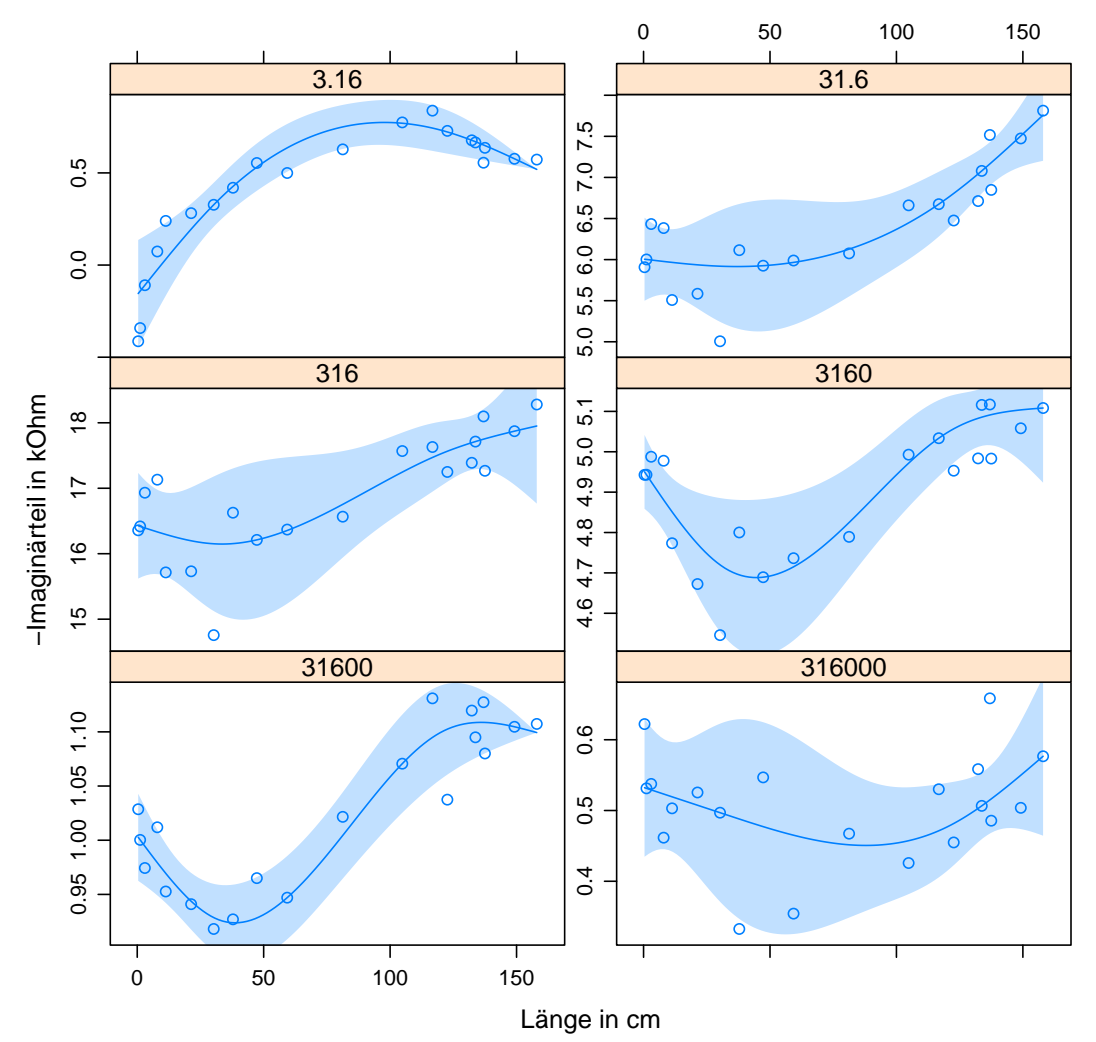

(b)

Abbildung 4.28: Zusammenhang zwischen Realteil der Impedanz u. Wurzellänge (a) und Imaginärteil der Impedanz und Wurzellänge (b) bei jeweils sechs verschiedenen Frequenzen in $\mathrm{Hz}$ an Steckling 9 aus Hauptversuch 1. 
In Abbildung 4.28b sticht zunächst die erste Teilgrafik $(3,16 \mathrm{~Hz})$ hervor. Der Verlauf der Ausgleichsfunktion unterscheidet sich deutlich von denen der anderen.

Während der Zusammenhang zwischen Imaginärteil der Impedanz und der Wurzellänge bei den Frequenzen $31,6 \mathrm{~Hz}-316000 \mathrm{~Hz}$ zunächst ebenso wie beim Realteil der Impedanz negativ ist, ist in der ersten Teilgrafik der Zusammenhang bis zu einer Wurzellänge von $100 \mathrm{~mm}$ positiv. Erst ab einer Wurzellänge von $>100 \mathrm{~mm}$ nimmt die Ausgleichsfuktion eine negative Steigung an. Beachtet werden muss hier, dass der Imaginärteil der Impedanz z. T. negative Werte aufgrund positiver Phasenwinkel annimmt. Dieser Befund wird als Messfehler gewertet (Abschnitt 4.3.1.

Auffällig ist weiterhin, dass der Vertrauensbereich der Teilgrafik bei der höchsten Frequenz $(316000 \mathrm{~Hz})$ am weitesten ist. Hier zeigt sich die größte Streuung der Messwerte.

Nachfolgend sind die Rohdaten des Realteils und des Imaginärteils der Impedanz mit der Wurzellänge für alle 14 Stecklinge korreliert worden. Das zugrundeliegende Modell entspricht dem aus Abbildung 4.28 .

In Tabelle 4.1 befinden sich die Bestimmtheitsmaße $\left(r^{2}\right)$ für die Betrachtung des Zusammenhanges zwischen Realteil und der Wurzellänge bzw. Imaginärteil und der Wurzellänge eines jeden Stecklings bei den bereits genannten sechs verschiedenen Frequenzen. Insgesamt liegen die Bestimmtheitsmaße bei Betrachtung des Zusammenhanges zwischen Realteil der Impedanz und Wurzellänge zwischen 0,14 und 0,99. Aufgrund der überwiegend hohen Bestimmtheitsmaße scheint die gewählte Splinefunktion ein passendes Modell für den Zusammenhang der betrachteten Parameter zu sein. Das Signifikanzniveau eines jeden betrachteten Zusammenhangs kommt in dieser Tabelle durch die verschiedenen Zellfarben zum Ausdruck.

Die Zusammenhänge zwischen Realteil der Impedanz und Wurzellänge bzw. Imaginärteil der Impedanz und Wurzellänge sind in den meisten Fällen höchstsignifikant $(0<\mathrm{p}$-Wert $<0,001)$.

Betrachtet man die Bestimmtheitsmaße frequenzweise, so fällt die höchste Frequenz dieses Kollektivs $(316,200 \mathrm{kHz})$ auf. Die Bestimmtheitsmaße sind etwas geringer als die der anderen untersuchten Frequenzen.

Die Bestimmtheitsmaße des Zusammenhangs zwischen Imaginärteil der Impedanz und der Wurzellänge zeigen ebenfalls überwiegend hohe Werte. Auffällig ist auch hier, dass die Bestimmtheitsmaße bei der höchsten Frequenz dieses Kollektivs $(316,200 \mathrm{kHz})$ geringer sind. Teilweise ist die statistische Berechnung zu keinem Ergebnis gekommen (-).

$\mathrm{Zu}$ keinem Ergebnis ist auch die Berechnung der Bestimmtheitsmaße von Steckling 15 für einige Zusammenhänge gekommen. An dieser Stelle sei darauf verwiesen, dass dieser Steckling als letzter Wurzeln ausgetrieben hat und damit wenige Daten für die statistische Auswertung zur Verfügung standen (vgl. Abbildung 4.23). 
Tabelle 4.1: Statistische Auswertung der Rohdaten von Hauptversuch 1; in den Zellen befinden sich die Bestimmtheitsmaße $\left(r^{2}\right)$ für die Betrachtung des Zusammenhanges zwischen Realteil und der Wurzellänge bzw. Imaginärteil und der Wurzellänge. Betrachtet werden 14 Stecklinge (St. 1-7 und 9-15) bei sechs verschiedenen Frequenzen.

Farbig dargestellt sind die Werte, bei denen das Signifikanzniveau $\geq 90 \%$ ist. Dabei wurde folgende Codierung getroffen: $0,05<\mathrm{p}$-Wert $<0,1$; 0,01<p-Wert $<0,05 ; 0,001<$ p-Wert $<0,01 ; 0<$ p-Wert $<0,001$.

\begin{tabular}{lllllll}
\hline Steckling & $3,162 \mathrm{~Hz}$ & $31,620 \mathrm{~Hz}$ & $316,200 \mathrm{~Hz}$ & $3,162 \mathrm{kHz}$ & $31,620 \mathrm{kHz}$ & $316,200 \mathrm{l}$ \\
\hline \multicolumn{5}{l}{ Für den Realteil der } & Impedanz & \multicolumn{2}{l}{ d die Wurzellänge } & & \\
1 & 0,88 & 0,86 & 0,72 & 0,85 & 0,80 & 0,73 \\
2 & 0,68 & 0,63 & 0,83 & 0,58 & 0,40 & 0,38 \\
3 & 0,44 & 0,64 & 0,93 & 0,68 & 0,72 & 0,42 \\
4 & 0,44 & 0,45 & 0,20 & 0,59 & 0,69 & 0,58 \\
5 & 0,71 & 0,73 & 0,90 & 0,80 & 0,69 & 0,61 \\
6 & 0,91 & 0,90 & 0,90 & 0,89 & 0,89 & 0,81 \\
7 & 0,90 & 0,90 & 0,95 & 0,89 & 0,88 & 0,88 \\
9 & 0,73 & 0,76 & 0,77 & 0,84 & 0,81 & 0,82 \\
10 & 0,95 & 0,96 & 0,97 & 0,89 & 0,90 & 0,81 \\
11 & 0,93 & 0,93 & 0,92 & 0,92 & 0,87 & 0,85 \\
12 & 0,93 & 0,94 & 0,48 & 0,48 & 0,88 & 0,87 \\
13 & 0,44 & 0,43 & 0,49 & 0,76 & 0,74 & 0,71 \\
14 & 0,83 & 0,83 & 0,84 & 0,82 & 0,79 & 0,74 \\
15 & - & 0,69 & - & 0,98 & 0,90 & 0,85
\end{tabular}

Für den Imaginärteil der Impedanz und die Wurzellänge

\begin{tabular}{llllll|l}
1 & 0,88 & 0,87 & 0,88 & 0,90 & 0,63 & 0,42 \\
\cline { 2 - 2 } 2 & 0,43 & 0,71 & 0,68 & 0,72 & 0,87 & - \\
3 & 0,76 & 0,33 & 0,73 & 0,90 & 0,66 & - \\
4 & 0,83 & 0,69 & 0,40 & 0,49 & 0,59 & - \\
5 & 0,95 & 0,66 & 0,76 & 0,92 & 0,80 & 0,14 \\
6 & 0,86 & 0,91 & 0,89 & 0,95 & 0,80 & - \\
\hline & 0,85 & 0,89 & 0,89 & 0,94 & 0,94 & 0,60 \\
9 & 0,98 & 0,80 & 0,71 & 0,80 & 0,88 & 0,28 \\
10 & 0,90 & 0,91 & 0,99 & 0,89 & 0,82 & - \\
11 & 0,53 & 0,94 & 0,92 & 0,96 & 0,95 & 0,85 \\
12 & 0,90 & 0,87 & 0,93 & 0,97 & 0,99 & 0,98 \\
13 & 0,69 & 0,45 & 0,37 & 0,63 & 0,70 & 0,78 \\
14 & 0,72 & 0,84 & 0,81 & 0,87 & 0,85 & 0,83 \\
15 & 0,80 & - & 0,86 & - & 0,82 & 0,83 \\
\hline
\end{tabular}


Hauptversuch 2 gliedert sich, wie beschrieben, in verschiedene Teilmessungen. Dargestellt werden daher Daten ausgewählter Stecklinge. Zunächst wird der Verlauf eines am Ende des Versuchszeitraumes besonders stark bewurzelten Stecklings (Steckling 3) in Dreipunktmessung dargestellt. Diese Daten sind Rohdaten. Die angesprochene Differenzbildung wird erst in der komplexen Ebene in Abbildung 4.31 berücksichtigt. So ist es möglich, einen Vergleich zum Hauptversuch $1 \mathrm{zu}$ erzielen, bei denen auch die Rohdaten in Dreipunktmessung dargestellt werden. Betrachtet man die Graphen dieser ausgewählten Frequenzen in Abbildung 4.29, so sind diese auch von deutlichen Tagesgängen gekennzeichnet. Durch die unterschiedliche Skalierung der Ordinaten beider Abbildungen treten die Temperatureffekte noch deutlicher hervor als beim Hauptversuch 1 .

Weiterhin fällt auf, dass die Messwerte des Hauptversuchs 2 deutlich niederohmiger als die des Hauptversuches 1 sind. Dies macht sich besonders bei den niedrigen Frequenzen im Realteil der Impedanz bemerkbar. Während beim Hauptversuch 2 bei den niedrigen Frequenzen Realteile von bis zu $18 \mathrm{k} \Omega$ erreicht werden, so wird beim Hauptversuch 1 der sechsfache Wert erreicht. Auch im mittleren und hohen Frequenzbereich ist dieser Effekt auffällig, jedoch fällt die Differenz wesentlich geringer aus (Frequenzen $>300 \mathrm{~Hz}$ ). Auch die Imaginärteile der Messwerte aus Hauptversuch 1 sind deutlich höher.

Im Gegensatz zu Hauptversuch 2 ist der Imaginärteil im niederfrequenten Bereich nahezu 7fach erhöht. Anders als beim Realteil ist dieser Effekt auch bei den hohen Frequenzen deutlich. Bei beiden Versuchen treten bei der in diesem Kollektiv niedrigsten Frequenz erneut positive Phasenwinkel auf, die zu einer scheinbaren Induktivität führen (siehe dazu Abschnitt 4.3.1).

Bei genauer Betrachtung ist auch bei diesem Steckling eine Einlaufphase zu erkennen, die ca. bis zum 21.07.2012 andauert (1. farblich hinterlegte Fläche). Der Zeitpunkt des ersten erkennbaren Auftretens von Wurzeln und dem anschließendem Wachstum ist hier ab dem 25.07.2012 im Diagramm visualisiert (2. farblich hinterlegte Fläche).

Der Bereich des Wurzelwachstums bei dem hier exemplarisch vorgestellten Steckling ist durch ein starkes Schwanken besonders beim Realteil gekennzeichnet. Diese Schwankungen sind sehr heterogen und dadurch schwer interpretierbar. Ein Trend ist insgesamt sowohl beim Realteil, als auch beim Imaginärteil nur schwer zu finden. Lediglich bei den Frequenzen $31,620 \mathrm{kHz}$ und $316,200 \mathrm{kHz}$ ist zumindest im Realteil ein Trend hin zu niedrigen Werten erkennbar.

Der Zusammenhang zwischen Realteil und Wurzellänge resp. Imaginärteil und Wurzellänge ist in Abbildung 4.30 auch für Hauptversuch 2 bei sechs verschiedenen Frequenzen aufgeführt. Zunächst werden hier die Rohdaten aus der Dreipunktmessung gezeigt.

In Abbildung 4.30a zeigt sich bei Betrachtung des Zusammenhangs zwischen Realteil der Impedanz und der Wurzellänge zunächst, dass die verschiedenen Graphen in den Einzelabbildungen keinen einheitlichen Trend haben. Allerdings lassen sich Funktionsgraphen mit annähernd ähnlichem Verlauf finden. Der Zusammenhang zwischen Realteil der Impedanz und Wurzellänge ist beispielsweise bei $3,16 \mathrm{kHz}$ und $316 \mathrm{~Hz}$ bis zu einer Wurzellänge von $22 \mathrm{~cm}$ zunächst negativ, anschließend lässt sich ein positiver Trend erkennen (bis ca. $38 \mathrm{~cm}$ Wurzellänge), danach schließt sich erneut eine negative Steigung an. Einen annähernd linearen Verlauf lässt sich bei der Grafik von 31,6 Hz erkennen. 
4.3 Impedanzspektroskopie

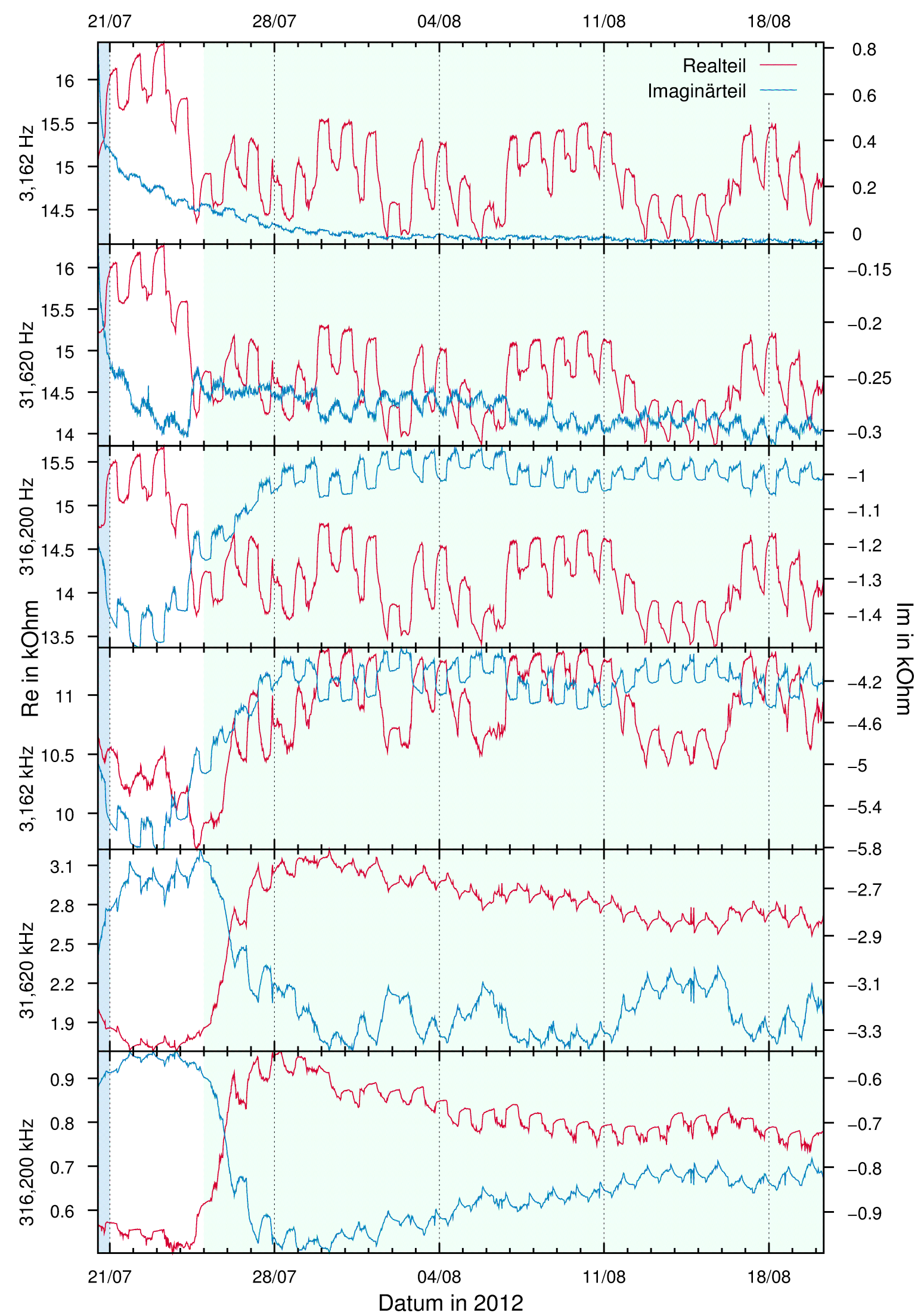

Abbildung 4.29: Verlauf von Real- und Imaginärteil von Steckling 3 bei sechs verschiedenen Frequenzen über der Zeit von Hauptversuch 2. 
Ein gegensätzliches Bild zeigen die Abbildungen der Frequenzen $3160 \mathrm{~Hz}$ und $31600 \mathrm{~Hz}$ sowie 316000 Hz.Hier ist zuerst ein positiver, danach ein negativer Trend erkennbar.

Auffällig ist weiterhin, dass die Vertrauensbereiche besonders bei den Teilgrafiken bei $3,16 \mathrm{~Hz}$, $31,6 \mathrm{~Hz}$ und $316 \mathrm{~Hz}$ der Abbildung 4.30a sehr weit sind. Dieser Befund zeigt sich im direkten Vergleich der Vertrauensbereiche bei den Zusammenhängen zwischen Realteil der Impedanz und der Wurzellänge bei Abbildung 4.28a (Hauptversuch 1), bei denen der Vertrauensbereich deutlich enger ist.

Betrachtet man den Zusammenhang zwischen Imaginärteil der Impedanz und Wurzellänge, ergibt sich beim Vergleich der unterschiedlichen Funktionsgraphen ein ebenso heterogenes Bild wie beim Realteil der Impedanz. Die Verläufe der Graphen unterscheiden sich zum Teil erheblich voneinander. Die Vertrauensbereiche hier sind im Gegensatz zu denen aus Abbildung 4.30a deutlich enger. Besonders die Teilabbildung für die niedrigste Frequenz dieses Kollektivs $(3,16 \mathrm{~Hz})$ sticht durch ihren positiven, annähernd linearen Trend hervor.

Auch in diesem Versuch wurden die Bestimmtheitsmaße für alle sieben Stecklinge bei den bereits genannten sechs Frequenzen für den Realteil der Impedanz wie den Imaginärteil der Impedanz errechnet und in Tabelle 4.2 dargestellt. Farblich hervogehoben sind die Bestimmtheitsmaße, die einen p-Wert von $\leq 0,1$ haben. Das zugrundeliegende Modell dieser Untersuchung entspricht dem aus Hauptversuch 1 (vgl. Tabelle 4.1)

Die Bestimmtheitsmaße liegen beim Zusammenhang zwischen Realteil resp. Imaginärteil der Impedanz und der Wurzellänge zwischen 0 und 0,99. Es ist nicht erkennbar, dass die Zusammenhänge bei einer bestimmten Frequenz besonders stark sind. Jedoch scheinen bestimmte Stecklinge über alle Frequenzen hinweg deutlichere Zusammenhänge zu zeigen als andere. So ergeben sich beispielsweise bei Steckling 7 sehr hohe $r^{2}$. Zudem sind die Zusammenhänge bei allen betrachteten Frequenzen hochsignifikant. Bei Steckling 1 ergeben sich eher schwache Zusammenhänge. Die Bestimmtheitsmaße sind deutlich geringer als bei Steckling 7 und die p-Werte deutlich höher.

Die Bestimmtheitsmaße für den Zusammenhang zwischen Imaginärteil der Impedanz und Wurzellänge sind insgesamt größer als beim Zusammenhang zwischen Realteil der Impedanz und der Wurzellänge. Es ergeben sich weiterhin erhöhte Signifikanzen. Insgesamt sind die Bestimmtheitsmaße der Zusammenhänge dieses Versuches jedoch geringer als beim ersten Hauptversuch.

Um eine Vergleichbarkeit der verschiedenen Messanordnungen dieses Versuches zu erzielen, werden in Abbildung 4.31 drei aufeinanderfolgende Messungen an Steckling 3 vom 23.07.2012 um ca. 13:30 Uhr in der komplexen Ebene dargestellt. Bei der ersten Messung werden die Daten aus der Dreipunktmessung dargestellt. Bei der Zweipunktmessung „oben“ wird lediglich die obere Stecklingselektrode, bei der Zweipunktmessung „unten“ lediglich die untere Stecklingselektrode mit in die Messung einbezogen. Der Graph „Zweipunktmessung mit Differenzbildung (unbewurzelt)“ zeigt die durch Differenzbildung veränderten Daten der Zweipunktmessung „unten“. Außerdem findet ein weiterer, durch Differenzbildung veränderter sweep vom 10.08.2012 um ca. 13:30 Uhr Berücksichtigung. Zu diesem Zeitpunkt war der hier angesprochene Steckling bereits bewurzelt (siehe Abbildung 4.25). 


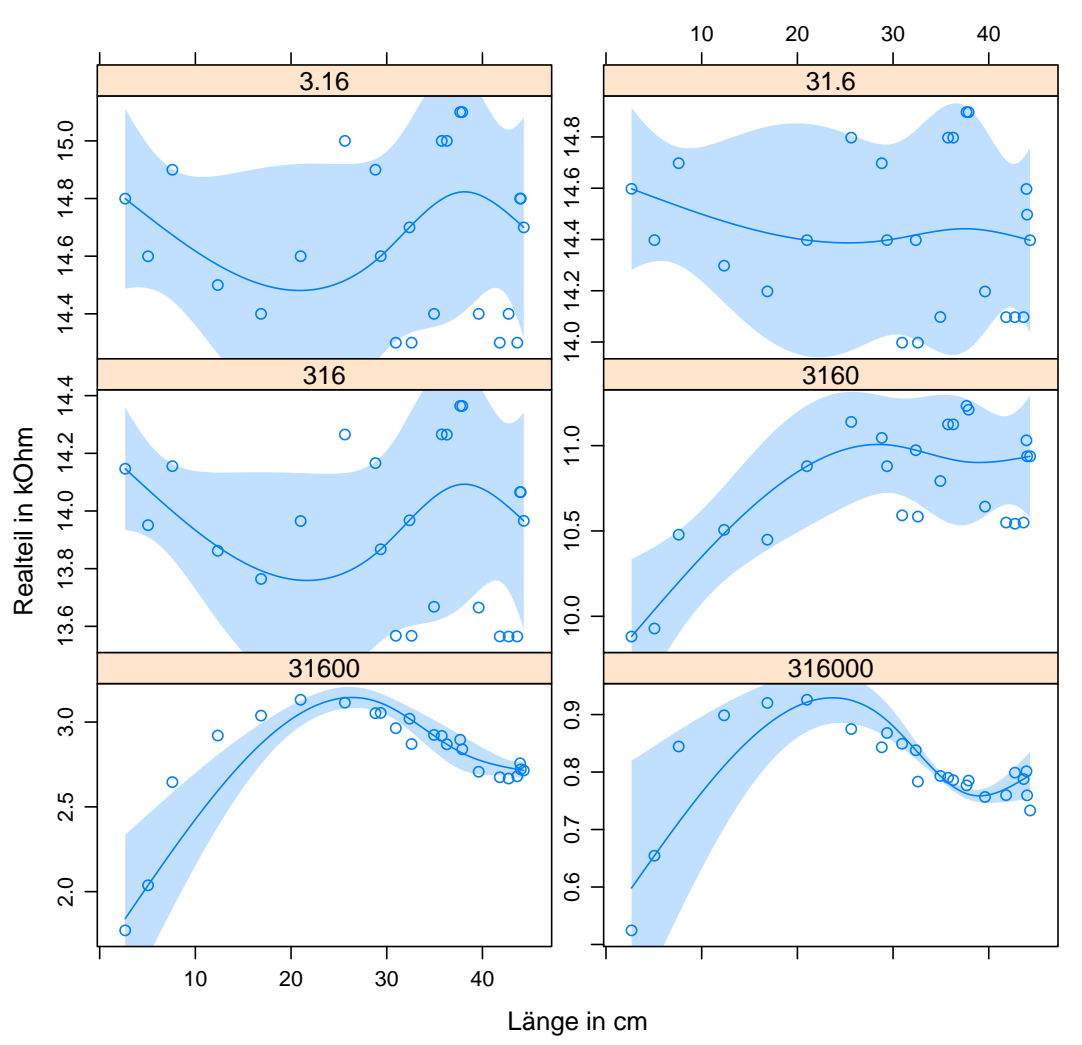

(a)

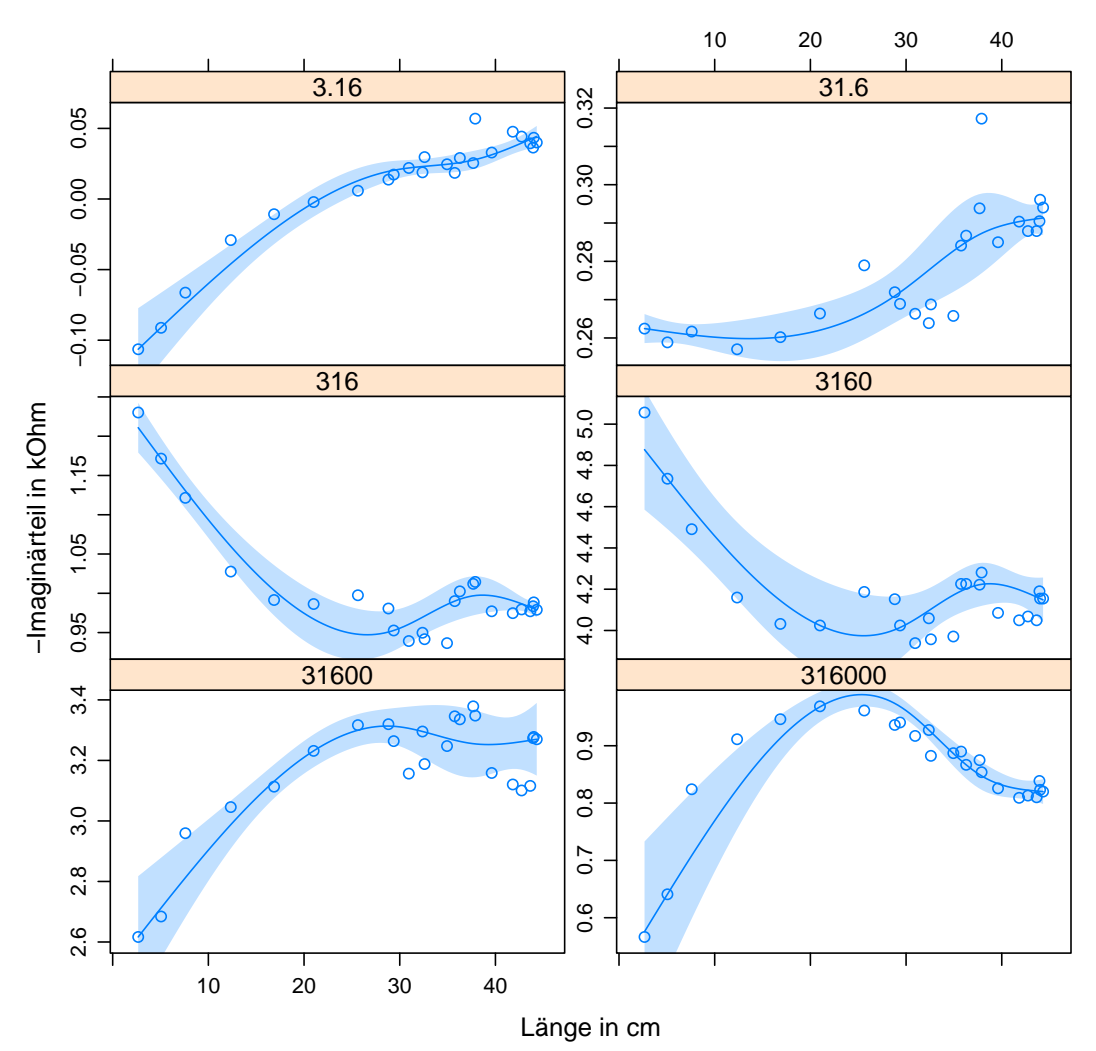

(b)

Abbildung 4.30: Zusammenhang zwischen Realteil der Impedanz und Wurzellänge (a) und Imaginärteil der Impedanz und Wurzellänge (b) bei jeweils sechs verschiedenen Frequenzen in $\mathrm{Hz}$ an Steckling 3 aus Hauptversuch 2. 
Tabelle 4.2: Statistische Auswertung der Rohdaten von Hauptversuch 2; in den Zellen befinden sich die Bestimmtheitsmaße $\left(r^{2}\right)$ für die Betrachtung des Zusammenhanges zwischen Realteil und der Wurzellänge bzw. Imaginärteil und der Wurzellänge. Betrachtet werden 7 Stecklinge bei sechs verschiedenen Frequenzen.

Farbig dargestellt sind die Werte, bei denen das Signifikanzniveau $\geq 90 \%$ ist. Dabei wurde folgende Codierung getroffen: $0,05<\mathrm{p}$-Wert $<0,1$; $0,01<\mathrm{p}$-Wert $<0,05 ; 0,001<\mathrm{p}$-Wert $<0,01 ; 0<\mathrm{p}$-Wert $<0,001$.

\begin{tabular}{|c|c|c|c|c|c|c|}
\hline Steckling & $3,162 \mathrm{~Hz}$ & $31,620 \mathrm{~Hz}$ & $316,200 \mathrm{~Hz}$ & $3,162 \mathrm{kHz}$ & $31,620 \mathrm{kHz}$ & $316,200 \mathrm{kHz}$ \\
\hline \multicolumn{7}{|c|}{ Für den Realteil der Impedanz und die Wurzellänge } \\
\hline 1 & 0,45 & 0,44 & 0,44 & 0,28 & 0 & 0 \\
\hline 2 & 0,38 & 0,40 & 0,39 & 0,44 & 0,90 & 0,86 \\
\hline 3 & 0,38 & 0,42 & 0,48 & 0,83 & 0,98 & 0,96 \\
\hline 4 & 0,33 & 0,33 & 0,35 & 0,28 & 0,13 & 0,33 \\
\hline 5 & 0,90 & 0,86 & 0,90 & 0,86 & 0,86 & 0,92 \\
\hline 6 & 0,33 & 0,34 & 0,35 & 0,35 & 0,46 & 0,40 \\
\hline 7 & 0,95 & 0,96 & 0,95 & 0,99 & 0,99 & 0,99 \\
\hline \multicolumn{7}{|c|}{ Für den Imaginärteil der Impedanz und die Wurzellänge } \\
\hline 1 & 0,75 & 0,48 & 0,14 & 0,12 & 0 & 0 \\
\hline 2 & 0,52 & 0,79 & 0,97 & 0,80 & 0,93 & 0,83 \\
\hline 3 & 0,97 & 0,84 & 0,97 & 0,94 & 0,92 & 0,96 \\
\hline 4 & 0,28 & 0,43 & 0,48 & 0,72 & 0,90 & 0,28 \\
\hline 5 & 0,97 & 0,92 & 0,87 & 0,88 & 0,93 & 0,91 \\
\hline 6 & 0,54 & 0 & 0,35 & 0,37 & 0,42 & 0,42 \\
\hline 7 & 0,99 & 0,95 & 0,98 & 0,98 & 0,99 & 0,99 \\
\hline
\end{tabular}

Vergleicht man zunächst die drei nicht veränderten ersten Messungen, so fällt der deutliche Unterschied im Verlauf der Graphen zwischen den Drei- und Zweipunktmessungen auf. Dieser Effekt ist bereits in Abbildung 4.13 genannt worden und bestätigt sich bei diesem Hauptversuch ebenfalls über alle Stecklinge im gesamten Versuchszeitraum. Die beiden Kurven der unveränderten Zweipunktmessungen („oben“ und „unten“) sind lediglich parallel zueinander verschoben. Der Einfluss des Leitgewebes ist bereits in Abschnitt 4.3 .2 behandelt worden. Die Auswirkungen dieses Einflussfaktors sind für alle sieben Stecklinge mit gleichem Trend erkennbar.

Die durch Differenzbildung veränderten Daten unterscheiden sich zu den Rohdaten besonders in den höheren Frequenzbereichen ab ca. $100 \mathrm{kHz}$ (linker Bereich der Grafik). Während bei den Frequenzen $<100 \mathrm{kHz}$ die Kurve der veränderten Daten hinsichtlich Imaginär- und Realteil niederohmiger geworden und annähernd parallel verschoben ist, zeigt sich bei Frequenzen $>100 \mathrm{kHz}$ ein anderes Bild. Der Imaginärteil der veränderten Daten ist, ähnlich wie bei den niedrigeren Frequenzen, um ca. $10 \%$ niedriger als bei den Rohdaten. Der größte Un- 
terschied zwischen den veränderten Daten dieses Versuches zu den Rohdaten zeigt sich beim Imaginärteil der Impedanz. Auffällig ist, dass der Graph der veränderten Daten bei den zehn höchsten Frequenzen (linker Bereich) einen gegenläufigen Verlauf zu den Rohdaten beschreibt. Die Differenzbildung ist demnach besonders für den oberen Frequenzbereich wichtig.

Betrachtet man weiterhin die veränderten Daten aus den zwei verschiedenen Versuchzeiträumen, so wird deutlich, dass der bewurzelte Steckling sowohl hinsichtlich des Imaginärteils, als auch hinsichtlich des Realteils, deutlich niederohmiger wird. Allerdings ähneln sich die Graphen vom Verlauf her stark, so dass auch hier von einer Verschiebung der Daten des bewurzelten Stecklings in den niederohmigen Bereich gesprochen werden kann.

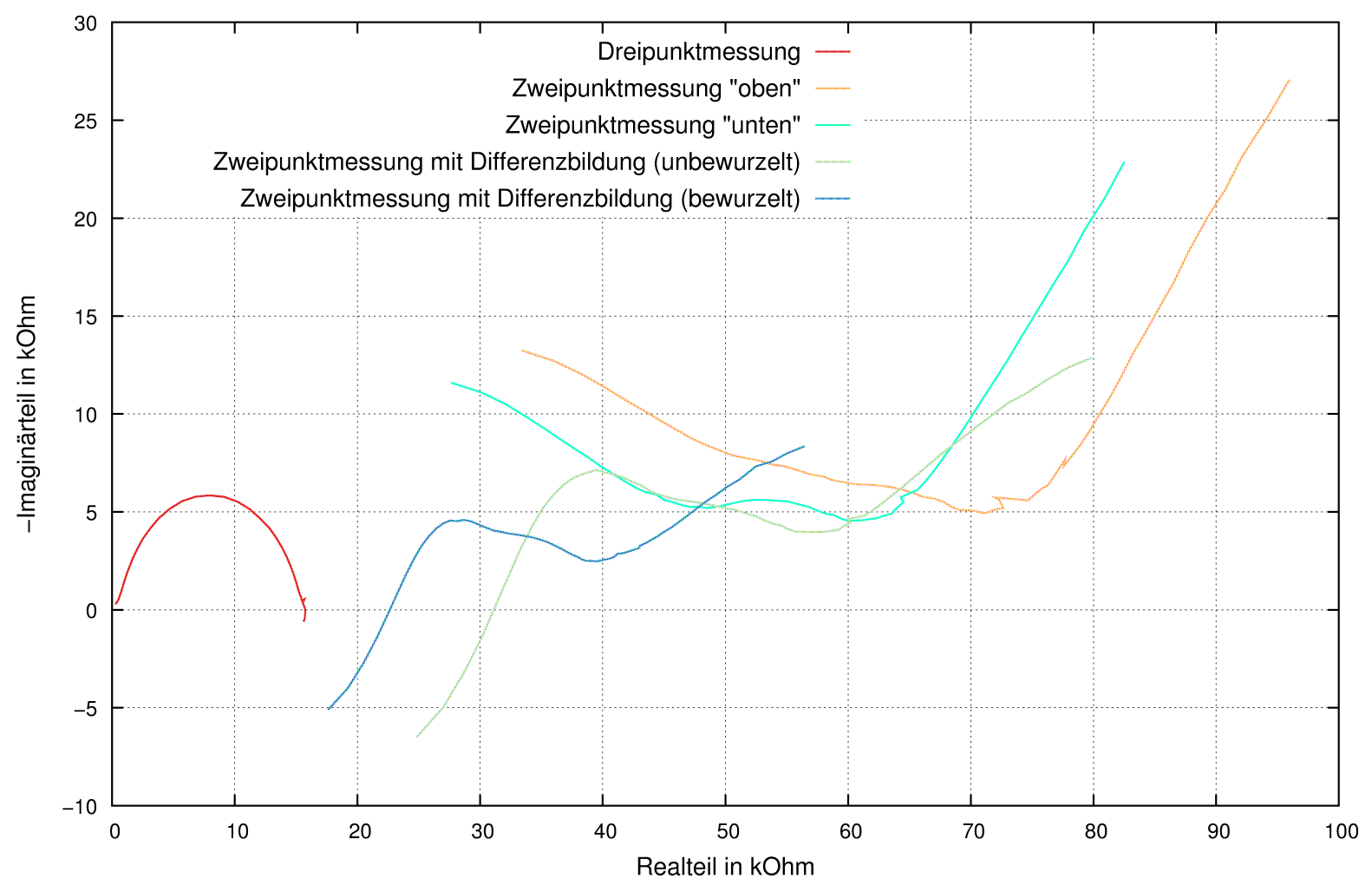

Abbildung 4.31: Ausgewählte Messergebnisse aus Hauptversuch 2 von Steckling 3 in der komplexen Ebene.

\section{Invertierung der Daten}

Um weiterführende Zusammenhänge zwischen den physikalischen und biologischen Eigenschaften der Stecklinge zu erhalten, werden nachfolgend die Ergebnisse der Invertierungen der Impedanzspektren aus Hauptversuch 1 und aus Hauptversuch 2 erläutert. Als Grundlage dient ein sweep eines jeden Tages in den jeweiligen Versuchszeiträumen. Alle sweeps der beiden Hauptversuche wurden aufgrund der bereits dargestellten tageszeitlichen Schwankungen zu einer ähnlichen Zeit (ca. 13:30 Uhr) ausgewählt. Diese Spektren wurden vor der Invertierung verändert, da im niederfrequenten Bereich, wie bereits erwähnt, teilweise positive Phasenwinkel und damit positive Imaginärteile auftraten. Diese scheinbaren Induktivitäten wurden bei der Modellbildung nicht berücksichtigt (siehe Abschnitt 5.3. 
Bei dem ersten Hauptversuch wurden im Versuchszeitraum vom 30.01.2011-24.02.2011 26 sweeps eines jeden Stecklings invertiert. Für die statistische Auswertung wurden allerdings nur sweeps verwendet, bei denen auch Daten der Wurzellänge vorlagen.

Es hat sich gezeigt, dass ein Modell, basierend auf einem Ersatzschaltbild mit einem vorgeschalteten Widerstand und vier RC-Gliedern, die ermittelten Messwerte an den Stecklingen gut abbildet (vgl. Abbildung 2.9).

Bei der Invertierung der Stecklingsdaten wurde getestet, ob durch eine Erweiterung des Modells aus Abbildung 2.9 um weitere RC-Glieder eine bessere Anpassung mit geringeren Fehlern möglich ist. Dabei wurde deutlich, dass beim überwiegenden Teil der Stecklinge nur marginale Verbesserungen erzielt werden konnten (kleine Residuen). Bei einigen Stecklingen (Stecklinge 6,9,13) konnte durch Anhängen von weiteren 1-2 RC-Gliedern eine wesentliche Verbesserung der Anpassung erzielt werden. Man entschied sich jedoch dafür, alle 14 Stecklinge mit dem gleichen Modell zu invertieren, da auf diese Weise die Modellparameter direkt miteinander vergleichbar sind. Durch die Wahl ähnlich dimensionierter Stecklinge und gleicher Versuchsbedingungen, ist die Anpassung der sweeps für jeden Steckling mit dem gleichen Modell zu rechtfertigen.

Beispielhaft wird in Abbildung 4.32 das Ergebnis der modellhaften Auswertung von Steckling 9 dargestellt.
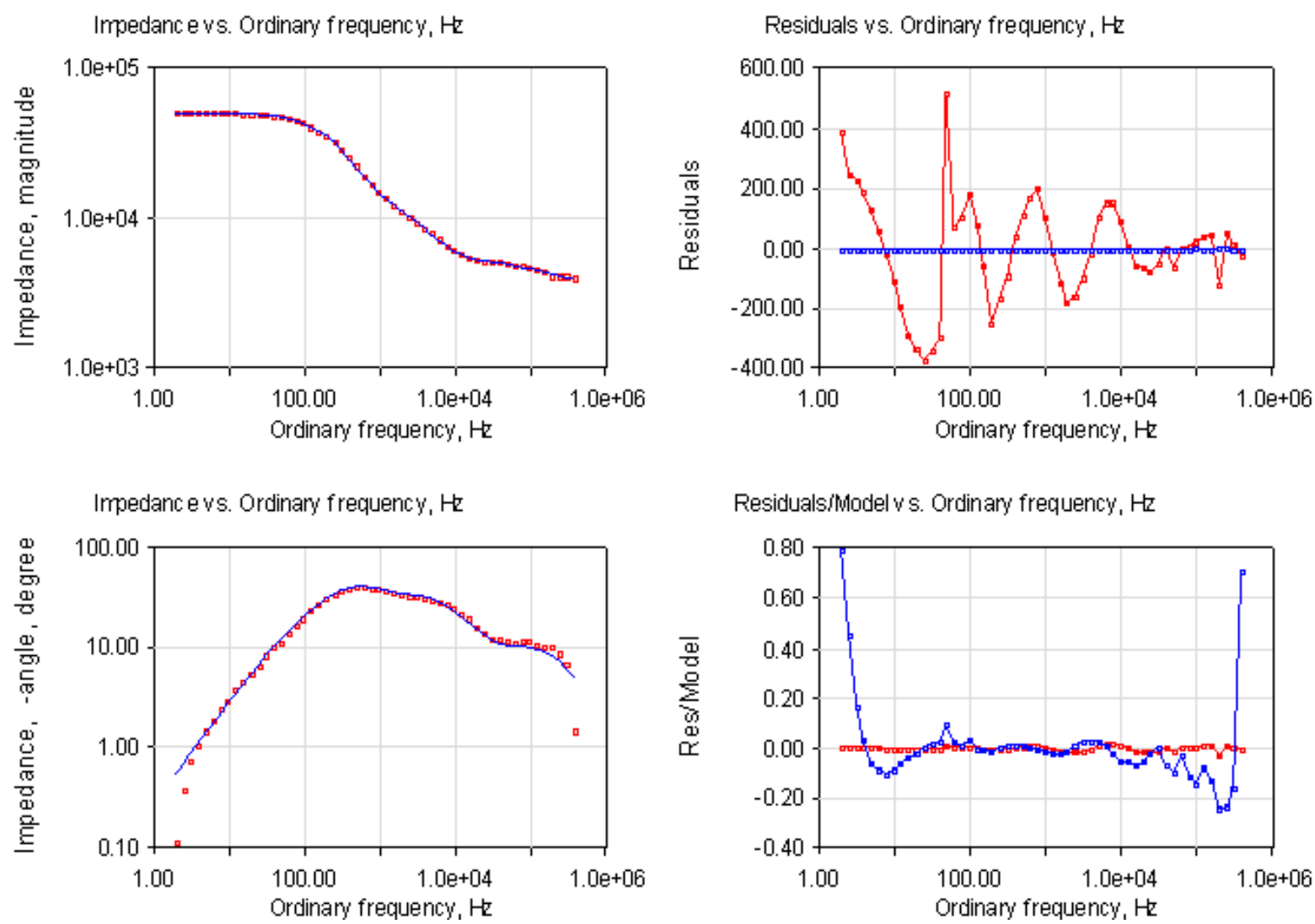

Abbildung 4.32: Darstellung von impedanzspektroskopischen Daten und Modelldaten eines sweeps von Steckling 9 vom 20.02.2011 (linke Seite) sowie die dazugehörigen Residuen (rechte Seite) aus dem Programm LEVM. 
Dieser Steckling und die dazugehörige Grafik entspricht einer durchschnittlichen Anpassung der Modelldaten an die Messwerte. Die statistischen Werte liegen unter Berücksichtigung des optimalen Modells bei allen ausgewerteten Stecklingen in ähnlichen Bereichen. In der Grafik oben links wird die Impedanz in $\Omega$ über der Frequenz dargestellt. Die roten Punkte stellen die Messwerte dieses sweeps dar, die durchgezogene blaue Linie zeigt die Modellkurve. Unten links werden die Messdaten der Phase (rote Punkte) und die dazugehörigen Modelldaten (blaue Linie) dargestellt.

Auffällig ist, dass die Messwerte in beiden Grafiken auf der linken Seite zum größten Teil auf der Modellkurve liegen. Lediglich in der Gafik unten links ist an der Peripherie, besonders bei den niedrigen Frequenzen, eine geringe Abweichung der Messdaten von den Modelldaten zu erkennen. In der oberen rechten Grafik sind die Residuen über der Frequenz und unten rechts der Quotient aus den Residuen und den Modelldaten dargestellt. Die Residuen liegen bei diesen beispielhaften Daten zwischen ca. -400 und $500 \Omega$. Bei der Betrachtung der Residuen fallen besonders die bereits oben angesprochenen peripheren Bereiche auf, in denen die Residuen besonders groß sind $\left(0,8^{\circ}\right)$. Insgesamt scheint das hier benutzte Modell die Messdaten gut zu erklären.

Bei der statistischen Betrachtung der Zusammenhänge zwischen Modelldaten und Wurzellänge wurde die multivariate Analyse gewählt, die eine statistische Aussage über die Zusammenhänge von Modelldaten und Wurzellänge über alle betrachteten Stecklinge ermöglicht. Weiterhin ist eine Aussage möglich, welche Modellparameter dem Objektparameter Wurzellänge zugeordnet werden können (vgl. Abbildung 2.6).

Bei der Auswertung der Modelldaten wurde hier, wie auch im Hauptversuch 2, auf die Betrachtung der Wurzeloberfläche verzichtet, da in Abbildung 4.24 gezeigt werden konnte, dass Wurzellänge und Wurzeloberfläche eng miteinander korrelieren. Durch den sehr engen Zusammenhang dieser beiden Größen kann bei der Betrachtung der Modelldaten und der Wurzeloberfläche von ähnlichen statistischen Größen ausgegangen werden.

Im Anhang (Abbildung A.10) ist das Ergebnis einer multivariaten Analyse durch ein generalisiertes additives Modell für die Betrachtung des Faktors Steckling sowie der Zusammenhänge zwischen den einzelnen Modellparametern mit der Wurzellänge dargestellt. Die dieser Untersuchung zugrunde liegenden Daten entstammen der Dreipunktmessung von 14 Stecklingen aus Hauptversuch 1 und wurden mit Hilfe des Modells (Ersatzschaltbild) aus Abbildung 2.9 invertiert. Als Bezugssteckling für die Betrachtung des Faktors Steckling wurde Steckling 1 herangezogen.

Hier ist auffällig, dass sich einige Stecklinge höchstsignifikant vom Bezugssteckling unterscheiden. Bei den Stecklingen 4, 5 und 9 ist das Signifikanzniveau hingegen gering (pWert $>0,1)$.

Weiterhin sind in dieser Abbildung Signifikanzen der Zusammenhänge von Wurzellänge und den verschiedenen Modellparameter ersichtlich. Die Modelldaten wurden durch ein generalisiertes additives Modell untersucht. Bei dieser Betrachtung fallen besonders die Modellparameter R0 und C1 auf. Bei beiden liegen höchstsignifikante Zusammenhänge zwischen der Wurzellänge und dem ohmschen Widerstand resp. der Kapazität vor (p-Wert $<0,001)$. Die anderen 


\section{Ergebnisse}

Modellparameter korrelieren nur schwach (C3) oder gar nicht mit der Wurzellänge (z. B. R1).

In Abbildung 4.33 sind die Zusammenhänge der verschiedenen Modellparameter mit der Wurzellänge in neun verschiedenen Einzelgrafiken dargestellt. Auf der Ordinate ist die Abweichung der Wurzellänge zum Referenzsteckling in cm aufgetragen, auf der Abszisse die Größen für die Wechselstromwiderstände. Die Widerstände der Parameter R0, R1, R2, R3, und R4 sind in $\Omega$, die Kapazitäten C1, C2, C3, C4 und C5 sind in F angegeben. Die durchgezogene Linie entspricht der Ausgleichskurve, die gestrichelten Linien markieren den Vertrauensbereich.

Hier wird deutlich, dass die beiden Einzelgrafiken für die Modellparameters C1 und R0 deutlich engere Vertrauensbereiche haben, als bei den anderen Einzelgrafiken. Beim Modellparameter R0 fällt auf, dass der Zusammenhang zwischen der Wurzellänge und dem ohmschen Widerstand annähernd positiv linear ist. Mit abnehmender Wurzellänge sinkt der ohmsche Widerstand ab. Bei der Kapazität (C1) folgt die Ausgleichskurve keinem linearen Trend. Die Kapazität nimmt mit abnehmender Wurzellänge tendenziell zu. 

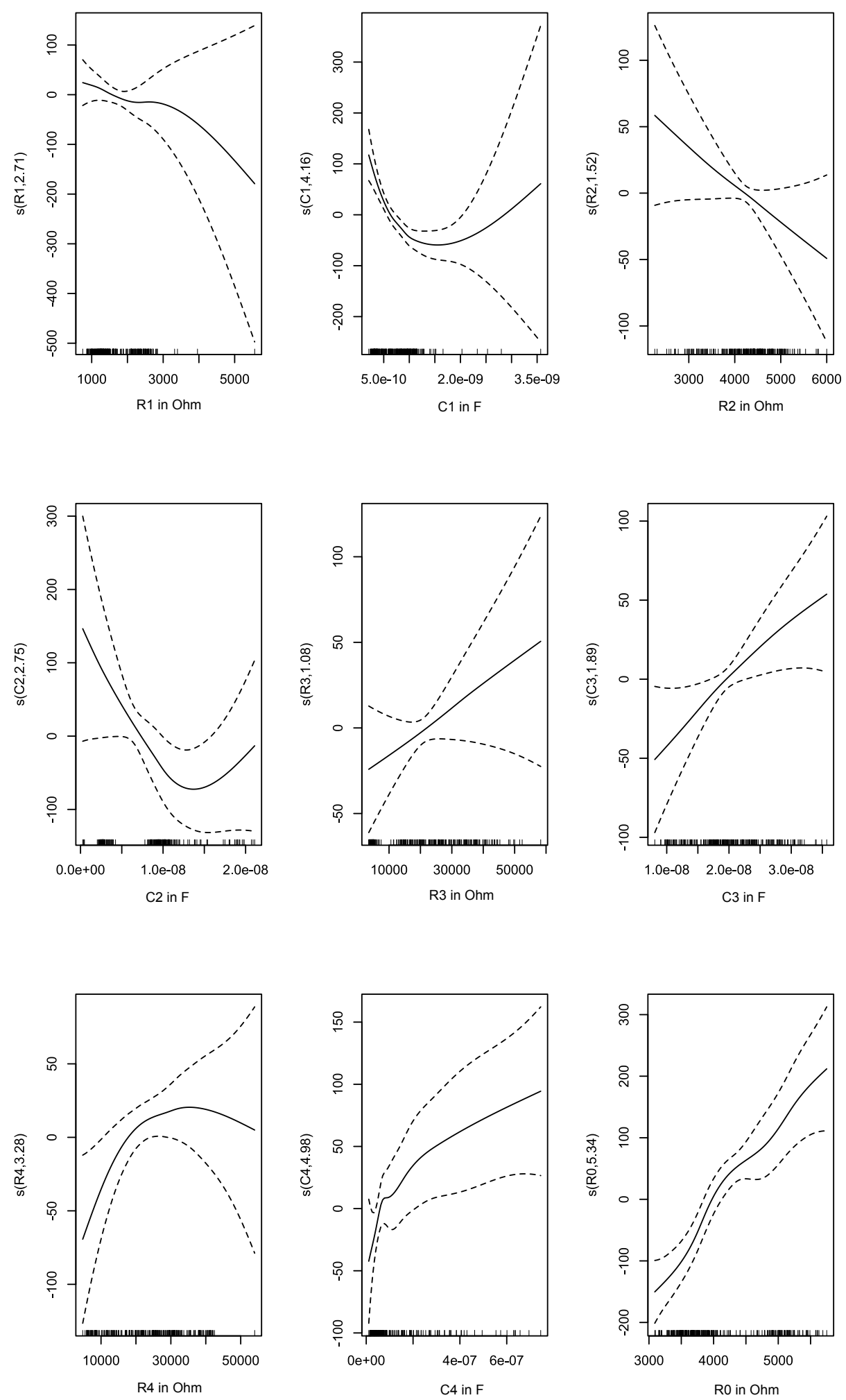

Abbildung 4.33: Darstellung der multivariaten Analyse von Hauptversuch 1 durch ein generalisiertes additives Modell. 
Für Hauptversuch 2 wurden zunächst die Daten durch die beschriebene Differenzbildung umgerechnet. Der Fokus soll auf die Zweipunktmessung unter Berücksichtigung der unteren Stecklingselektrode gelegt werden. Die Anpassung dieser Daten an verschiedene Modelle (Modelle aus Abbildung 2.8 ist in Abbildung 4.34 in der komplexen Ebene dargestellt. In dieser Grafik wird ein sweep von Steckling 3 und exemplarische ausgewählte Anpassungen der Modelle an diese Messdaten gezeigt. Die Daten entstammen vom 23.07.2012 um ca. 13:30 Uhr. $\mathrm{Zu}$ diesem Zeitpunkt war der Steckling unbewurzelt.

Auf der Ordinate ist der Imaginärteil der Impedanz (Z"), auf der Abszisse der Realteil der Impedanz (Z') aufgetragen. Die Einheit für sämtliche Messwerte ist $\Omega$. Die ersten beiden Einzelgrafiken dieser Abbildung zeigen die Anpassung der Daten zunächst im Frequenzbereich von $60 \mathrm{~Hz}$ bis $60 \mathrm{kHz}$ für das Modell in Abbildung 2.8a (1. Einzelgrafik) und das Modell in Abbildung 2.8b (2. Einzelgrafik). Es wird deutlich, dass bereits die Messwerte des unbewurzelten Stecklings besser mit dem Modell in Abbildung 2.8b erklärt werden können. Die Abweichung der Modellwerte zu den Messwerten sind hier niedriger. Die Residuen sind im zweiten Fall (2. Abbildung) niedriger als im ersten (1. Abbildung). Für die modellhafte Betrachtung und die anschließende Korrelation der Modellparameter mit den wurzelspezifischen Größen im bereits beschriebenen Frequenzbereich wird daher das Modell in Abbildung 2.8b angewandt.

Weiterhin zeigt sich, dass dieses Modell bei Erweiterung um die peripheren Bereiche jenseits $60 \mathrm{~Hz}$ bzw. $60 \mathrm{kHz}$ die Messdaten wiederum nicht gut beschreibt. Die modellhafte Betrachtung über den gesamten gemessenen Frequenzbereich von $1 \mathrm{~Hz}-10^{6} \mathrm{~Hz}$ ist in der dritten (Anpassung mit Modell in Abbildung 2.8b) und vierten Einzelgrafik (Anpassung mit dem Modell aus Abbildung 2.8b mit Erweiterung um einen ohmschen Widerstand und Kondensator in Serie) berücksichtigt. Hier zeigt sich, dass bei Modifizierung des Modells aus Abbildung 2.8b eine wesentlich bessere Angleichung erreicht werden kann. Die Residuen sind hier geringer.

Es wurde versucht, dieses neue Modell mit weiteren ohmschen Widerständen und Kondensatoren zu modifizieren. Diese Versuche bedeuteten jedoch ein wesentlich komplexeres Ersatzschaltbild bei ähnlich kleinen Residuen bzw. Fehlern, so dass dieses komplexere Modell nicht weiter verfolgt wurde.

Die letzte Einzelgrafik in dieser Abbildung zeigt die Modellierung des sweeps mit dem modifizierten Modell aus Abbildung 2.9. Bei Anpassung mit Hilfe dieses Modells und einer Erweiterung um zwei zusätzliche RC-Glieder in Serie konnten ähnlich kleine Residuen erreicht werden, wie bei der Anpassung mit einem Modell mit nur einem zusätzlich angehängten RC-Glied.

Bei der Modellierung wurden die veränderten Daten der sieben Stecklinge aus Hauptversuch 2 im Frequenzbereich von $60 \mathrm{~Hz}$ bis $60 \mathrm{kHz}$ berücksichtigt. Zudem wurden die nicht durch Differenzbildung umgerechneten Daten aus der Dreipunktmessung dieser Stecklinge im gesamten Frequenzbereich invertiert. Bei beiden Auswertungen wurde das Modell (Ersatzschaltbild) aus Abbildung 2.8b und die dortige Nomenklatur für die Wechselstromwiderstände (R1-C5) genutzt. 

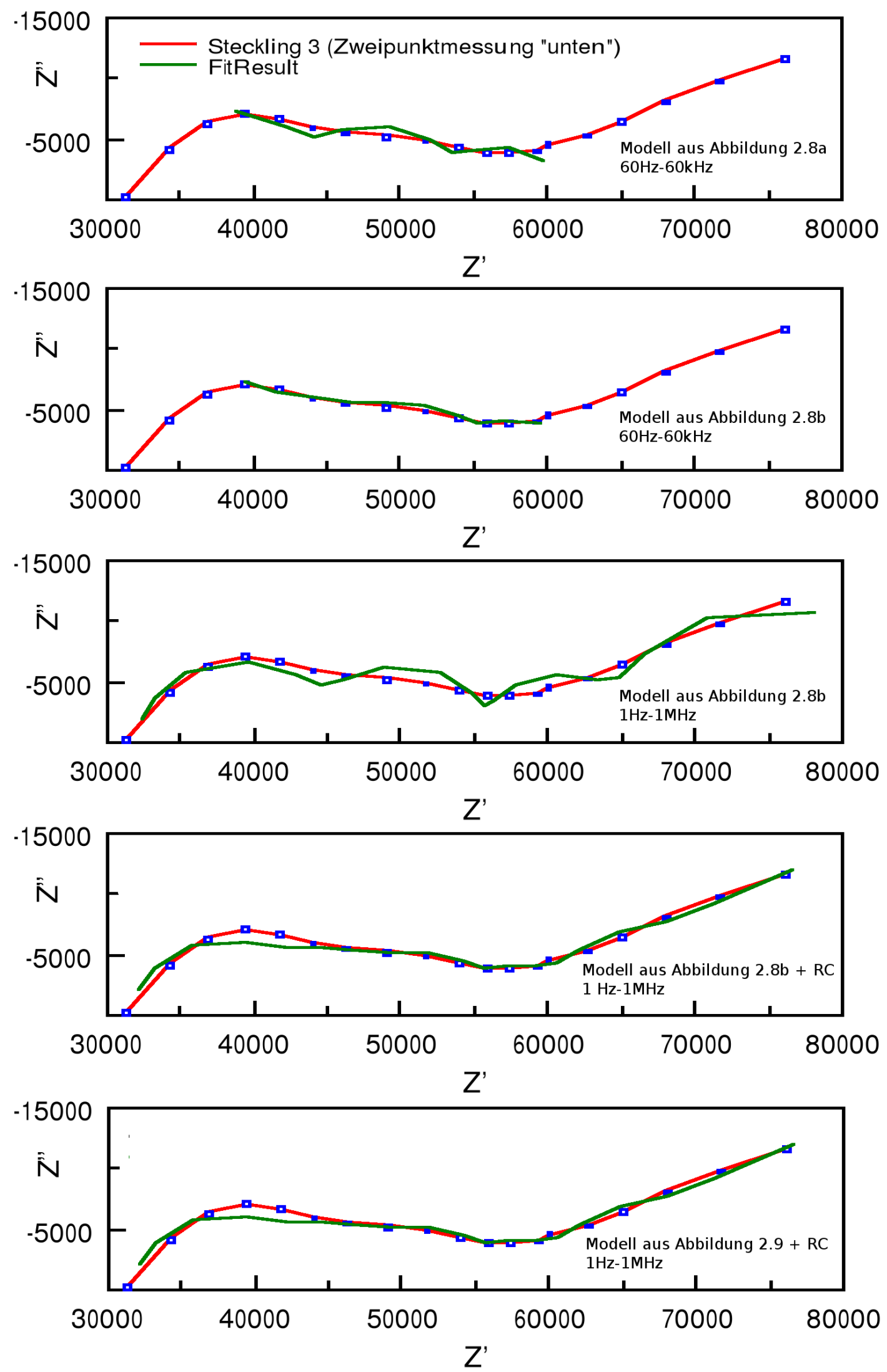

Abbildung 4.34: Darstellung von impedanzspektroskopischen Daten und Modelldaten eines sweeps von Steckling 3 vom 23.07.2012. Die genaue Beschreibung und die Unterschiede zwischen den Einzelgrafiken finden sich im Text. 
Die statistische Auswertung erfolgt auf gleiche Weise wie Hauptversuch 1. Die multivariate Analyse durch ein generalisiertes additives Modell ist für die neun verschiedenen Modellparameter in Abbildung 4.35 (Zweipunktmessung) und Abbildung 4.36 (Dreipunktmessung) ersichtlich. In Abbildung A.11 und Abbildung A.12 sind wichtige statistische Größen für diese Untersuchung tabellarisch zusammengefasst. Begonnen werden soll mit der Zweipunktmessung, anschließend folgt die Erläuterung der Ergebnisse der Dreipunktmessung.

Betrachtet man zunächst in Abbildung A.11 den Faktor Steckling, so fällt auf, dass sich einige Stecklinge von einander höchstsignifikant (Steckling 3,5 und 7) bzw. hochsignifikant voneinander unterscheiden (Steckling 2). Als Referenzsteckling wurde hier Steckling 1 gewählt. Weiterhin ist ersichtlich, dass die Zusammenhänge zwischen Wurzellänge und den neun verschiedenen Modellparametern außer beim Parameter C5 mindestens signifikant (z. B. C3), in vielen Fällen sogar höchstsignifikant (z. B. R1) sind. In Abbildung 4.35 sind die Zusammenhänge der neun verschiedenen Modellparameter mit der Wurzellänge in gleicher Weise wie bei Hauptversuch 1 grafisch dargestellt. Beim Blick auf die Zusammenhänge zwischen Wurzellänge und den verschiedenen Modellparametern (Abbildung 4.35) zeigt sich, dass die verschiedenen Modellparameter in unterschiedlicher Weise mit der Wurzellänge zusammenhängen. Die Kapazität C5 fällt, wie bereits durch die statistische Betrachtungsweise gesehen, aus dem Kollektiv heraus. Der Vertrauensbereich ist hier sehr groß und es ist kein Zusammenhang zwischen den untersuchten Größen erkennbar. C3 und C4 zeichnen sich ebenfalls durch einen weiten Vertrauensbereich aus und ähneln sich hinsichtlich ihres Verlaufs. Der annähernd horizontale Verlauf beider Ausgleichskurven lässt einen eher schwachen Zusammenhang schlussfolgern.

Der Trend der Ausgleichskurve bei Betrachtung des Zusammenhangs zwischen der Wurzellänge und der Kapazität bei Parameter C1 zeigt einen eindeutigeren Trend als bei den bereits beschriebenen Kapazitäten. Hier zeigt sich tendenziell, dass bei zunehmender Wurzellänge die Kapazitäten sinken.

Bei den ohmschen Widerständen ergibt sich ebenfalls ein heterogenes Bild. Insgesamt sind die Vertrauensbereiche allerdings enger als bei den Kapazitäten. Die Kurven für die Parameter R1 und R3 zeigen eher eine Zunahme des Widerstandes mit zunehmender Wurzellänge. Im niederohmigen Bereich kehrt sich der Trend allerdings um. Parameter R2 lässt ein eher gegensätzliches Bild erkennen. Der eindeutigste Trend ist bei Parameter R5 zu erkennen. Hier ist bis auf den niederohmigen Bereich ein gleichbleibender annähernd linearer Verlauf erkennbar. Mit zunehmender Wurzellänge steigt der ohmsche Widerstand.

Die statistische Auswertung der invertierten Messergebnisse ist in Abbildung A.12 für die Dreipunktmessung dargestellt. Bei Betrachtung des Faktors Steckling ergeben sich auch hier einige z. T. höchstsignifikante Unterschiede. Hier sticht besonders der Steckling 6 heraus. Bei der Analyse der Beziehungen zwischen Wurzellänge und Kapazität resp. ohmscher Widerstand fallen zwei Modellparameter heraus. C1 und C3 weisen p-Werte > 0,1 auf. Es lassen sich keine signifikanten Zusammenhänge erkennen. Auffällig ist, dass hier, wie auch bei der Zweipunktmessung dieses Versuches (Abbildung A.11), Kapazitäten nicht mit der Wurzelänge korrelieren. Anders als bei der Zweipunktmessung ergab die statistischen Auswertung der Daten der Dreipunktmessung, dass der Modellparameter C5 höchstsignifikant mit der Wurzellänge korreliert. 
Dieser Modellparameter korreliert allerdings bei der Dreipunktmessung nicht mit der Wurzellänge. Die Signifikanzniveaus der Modellparameter R2, R3, R4, C4 und C5 sind bei beiden Messvarianten dieses Versuches gleich.

Die angesprochenen schwachen Zusammenhänge zwischen Wurzellänge und Modellparameter $\mathrm{C} 1$ und $\mathrm{C} 3$ kann man auch in Abbildung 4.35 erkennen. Betrachtet man die anderen beiden Kapazitäten dieses Modells (C5 und C4), so ist hier der Trend erkennbar, dass, wie auch bei Parameter C1 der Zweipunktmessung (Abbildung 4.35), die Kapazität mit zunehmender Wurzellänge sinkt. Dabei ist ein annähernd linearer Trend bei C4 erkennbar. Die ohmschen Widerstände (R1-R5) hingegen zeigen einerseits, dass mit zunehmener Wurzellänge der Widerstand tendenziell steigt (R3 und R5), andererseits, dass mit abnehmender Wurzellänge der Widerstand eher sinkt (R1, R2 und R4). Besonders eindeutig sind die verschiedenen Zusammenhänge bei R1, R2 und R3. Hier erscheinen die Funktionen fast linear. 

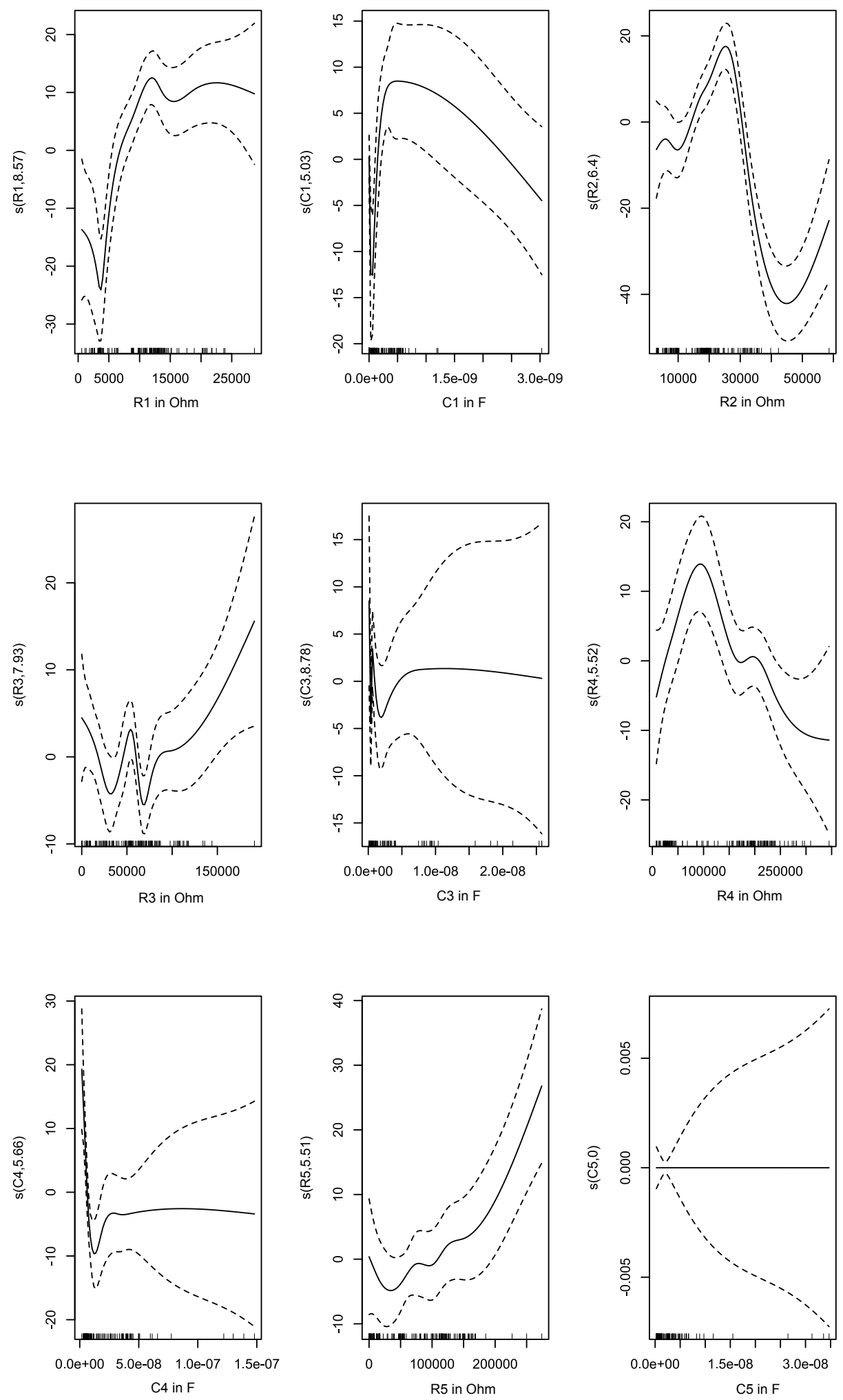

Abbildung 4.35: Darstellung der multivariaten Analyse von Hauptversuch 2 durch ein generalisiertes additives Modell. Invertiert wurden die Zweipunkt-Messdaten im Frequenzbereich $60 \mathrm{~Hz}$ bis $60 \mathrm{kHz}$, die zuvor durch Differenzbildung verändert wurden. 

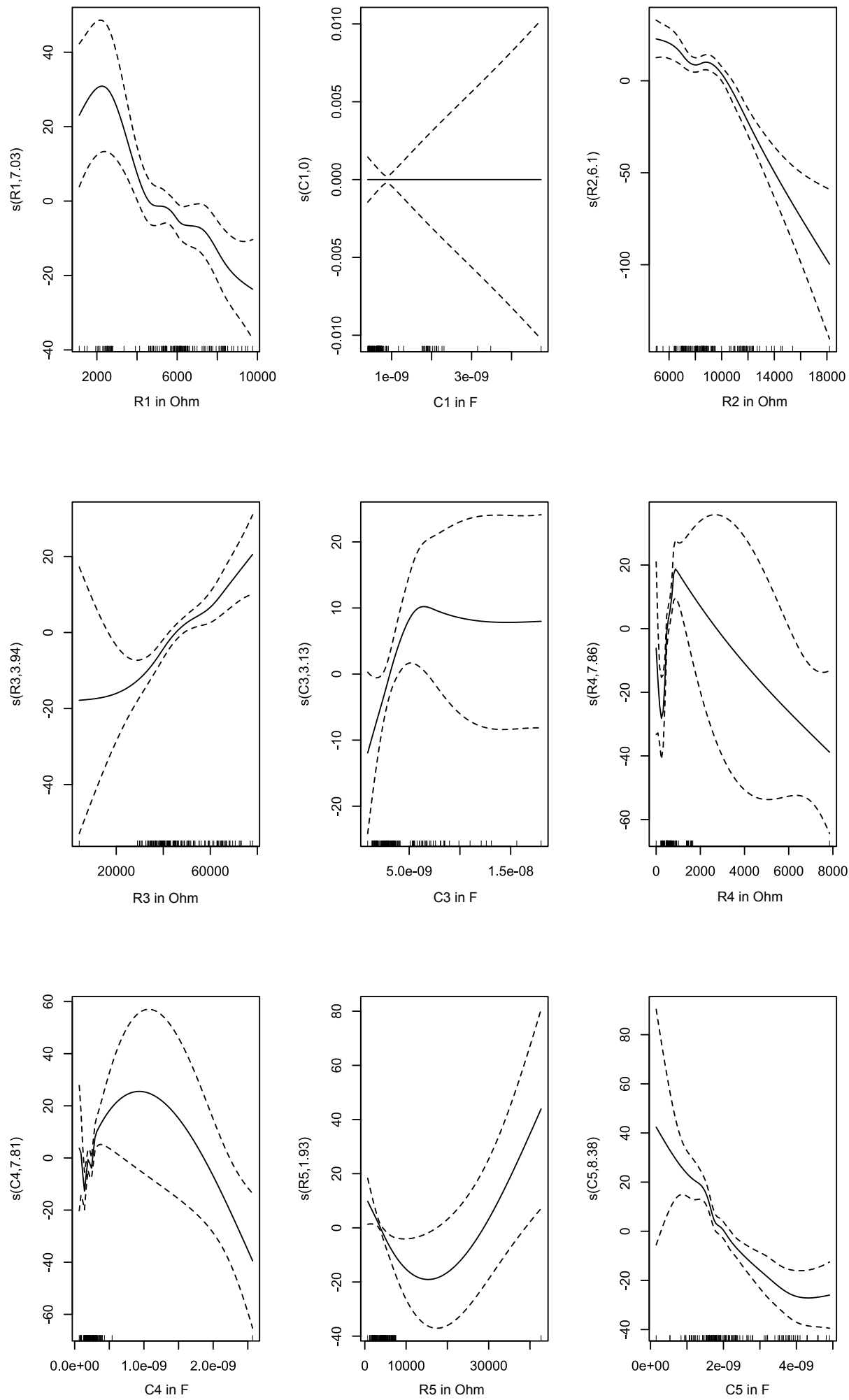

Abbildung 4.36: Darstellung der multivariaten Analyse von Hauptversuch 2 durch ein generalisiertes additives Modell. Invertiert wurden die Dreipunkt-Messdaten im gesamten Frequenzbereich ohne Veränderung der Daten durch Differenzbildung. 



\section{Diskussion}

\subsection{Erdungsmessung}

Die Zusammenhänge zwischen der Oberfläche eines Leiters und dem elektrischen Widerstand sind aus mathematisch-physikalischer Sichtweise unumstritten. Die anfänglichen Messungen beispielsweise von Yamaura u.a. (2000) machten bereits deutlich, dass eine direkte Übertragung der Erdungsmessung, als eine in der Geophysik etablierten Methode, auf Bäume nicht möglich ist. Übergangswiderstände sind aufgrund der unterschiedlichen Materialien unbedingt zu beachten und können in situ bei Vergleich der beiden Messanordnungen (Dreipunkt- und Vierpunktmessung) ermittelt werden.

Beim direkten Vergleich eines Dreipunktmesswertes mit nur einer Elektrode am Baum und dem Vierpunktmesswert (siehe Abbildung 4.2) wurde deutlich, dass der Dreipunktmesswert im Gegensatz zum Vierpunktmesswert um mehr als $400 \%$ höher ausfiel. Diese Versuchsreihe zeigte, dass der Dreipunktmesswert mit sukzessiv ansteigender Elektrodenanzahl keine korrekten Erdungsmesswerte liefert.

Die Differenz aus dem durch Dreipunktmessung mit anschließender Extrapolation und durch Vierpunktmessung ermittelten Erdungsmesswert von $6 \%$ ist durch die wenigen „Startwerte“ von lediglich neun Messwerten zu begründen. Außerdem war es messtechnisch nicht möglich, eine gleichbleibende Einschlagtiefe der neun Elektroden im Baum zu gewährleisten. Da die kontaktierte Oberfläche der Elektroden, wie erwähnt, im direkten Zusammenhang mit dem elektrischen Widerstand steht, ergeben sich bereits hier Ungenauigkeiten.

Insgesamt hat sich gezeigt, dass Übergangswiderstände nicht als Fixwert von jeder Dreipunktmessung in Differenz gebracht werden können. Aussagen dieser Dreipunktmessergebnisse sind demnach wenig aussagekräftig und fehlerbehaftet. Auch Hanskötter (2004) weist in ihrer Arbeit im Zusammenhang mit Messobjekten aus Holz und (Edelstahl-) Elektroden auf die stark schwankenden Übergangswiderstände während der Untersuchungen hin. Um korrekte Erdungsmessungen am Baum durchzuführen, ist daher eine Vierpunktmessung unumgänglich.

Messergebnisse, die einer Erdungsmessung in Vierleitermesstechnik entstammen, sind jedoch mit weiteren sensiblen Parametern behaftet. Betrachtet man Tabelle 2.1, so wird deutlich, dass beispielsweise der Schwankungsbereich des spezifischen elektrischen Widerstandes des Bodens zwischen drei Größenordnungen liegen kann. Der Boden bzw. das Substrat in dem die Messobjekte verwurzelt sind, sind integrale Bestandteile des Erdungswiderstandes. Dieser Befund wurde bereits von Yamaura u.a. (2000) beschrieben. Ausgehend von diesen Erkenntnissen, muss dieser Faktor bei vergleichenden Messungen entweder in Abzug gebracht werden, nachdem er durch eine separate Messung erfasst wurde, oder konstant gehalten werden. Letzteres ist bei Feldversuchen im Gelände jedoch nur schwer möglich. In dieser Arbeit sind die Messungen an 60 Linden zu ähnlichen Zeitpunkten auf vergleichbarem Substrat durchgeführt worden, 


\section{Diskussion}

so dass der Schwankungsbereich dieses Faktors in einstelligem Prozentbereich lag. Ein direkter Abzug dieses bodenspezifischen Wertes vom Erdungswiderstand gestaltet sich als schwierig, da der Einfluss dieser bodenspezifischen Größe auf den Gesamterdungswiderstand nicht bekannt ist. Aus diesem Grund können die Messungen zwar untereinander, jedoch nicht mit Werten bei anderen Versuchsbedingungen verglichen werden. Die Substrateigenschaften scheinen jedoch nicht den größten Anteil des Erdungswiderstandes auszumachen. Aubrecht u. a. (2006) weisen zwar darauf hin, dass der Wassergehalt bei der nah verwandten elektrischen Widerstandstomographie einer der wesentlichen Einflussfaktoren ist, bei der Erdungsmessung an Bäumen scheint dieser Faktor jedoch hinter anderen Einflussgrößen zurück zu stehen.

Bei den Untersuchungen von 60 Linden, wurde ein hochsignifikanter Zusammenhang zwischen dem Brusthöhendurchmesser (BHD) und dem Erdungswiderstand in zwei verschiedenen Höhen gefunden. Zunächst wurde dieser Zusammenhang mit der erhöhten Feinwurzelmasse (Oberfläche) von dimensionstärkeren Bäumen erklärt. Bei genauerer Untersuchung wird aber deutlich, dass bei einer Erdungsmessung mit Messelektroden am Stamm in $100 \mathrm{~cm}$ Höhe vom Erdboden ca. 90 \% (im Mittel 88\%) alleine durch den elektrischen Widerstand dieses $100 \mathrm{~cm}$ leitenden Holzgewebes zu erklären ist. Durchmesserstärkere Bäume leiten elektrischen Strom offenbar besser und erscheinen aus diesem Grund besser geerdet. Die erhöhte Feinwurzelmasse dickerer Bäume trägt offensichtlich nur gering dazu bei.

Wenn also eine Aussage über andere wesentliche baumspezifische Größen getroffen werden soll, müssen Messungen in jedem Fall bodennah erfolgen. Dennoch muss auch bei diesen Messungen der hohe elektrische Widerstand des Holzgewebes Berücksichtigung finden, denn unterhalb der Messelektrode $\mathrm{M}_{1}$ im Wurzelhalsbereich (E0) differenziert sich das Wurzelsystem weiter in Grob- und Feinwurzeln. Angesichts der Tatsache, dass nur $1 \mathrm{~m}$ Stamm (Leitgewebe) bis maximal $95 \%$ des Erdungswiderstandes ausmachen, wird dieser Bereich immer zu einer Überschätzung des Erdungswiderstandes führen.

Zusätzlich dazu muss beachtet werden, dass nicht nur der elektrische Widerstand des Stammes für diesen bodennah gemessenen Wert relevant ist. Da sich der Wurzelhalsbereich in dünnere Wurzelbereiche „teilt", muss ebenfalls der Zusammenhang zwischen Durchmesser und elektrischem Widerstand beachtet werden. Das heißt, dass sich das Wurzelsystem zusätzlich zu dem unterhalb der Bodenelektrode liegenden Leitgewebe in der Regel verjüngt. Durchmesserschwächere Bereiche haben, wie in dieser Arbeit gesehen, höhere elektrische Widerstände als durchmesserstärkere. Dieser unterirdische Bereich ist allerdings deutlich schwieriger in Abzug zu bringen, als der oberirdisch sichtbare und bestimmbare errechenbare ohmsche Widerstand des Stammes. Das Wurzelsystem beeinflusst den Erdungswiderstand also durch zwei Aspekte. Einerseits durch die Leitgewebe als solche, andererseits durch den kleineren Durchmesser dieses Gewebes.

Die Überlegungen zeigen, dass der eigentliche Erdungswiderstand, also der elektrische Widerstand, der zwischen Substrat und Wurzeloberfläche auftritt, ein so kleiner Wert ist, dass er durch die beschriebenen Einflussparameter erheblich maskiert wird. An dieser Stelle sei darauf hingewiesen, dass dieser Gedankengang auf der nicht näher untersuchten Annahme aus Aubrecht u. a. (2006) sowie Čermák u.a. (2006) beruht, dass die Exodermis von Wurzeln 


\subsection{Bodenwiderstandsmethode}

elektrisch isolierend wirkt. Auch können aus den mono- und niederfrequenten Untersuchungen lediglich geringe Aussagen zu kapazitiven Effekten getroffen werden. Im niederfrequenten Bereich von wenigen Hertz kommt zum großen Teil der Realteil der Impedanz zum Vorschein. Der Imaginärteil und damit die kapazitive Betrachtung ist hier nicht oder nur in geringem Maße möglich. Bei Yamada u. a. (2003) wird dieser Aspekt angesprochen, jedoch in Folgearbeiten auch nicht weiter untersucht.

\subsection{Bodenwiderstandsmethode}

Um sich dem Verfahren der Bodenwiderstandsmethode zu nähern, wurden, wie beschrieben, Modellversuche durchgeführt. An dieser Stelle sei darauf hingewiesen, dass sich der Versuchsaufbau im elektrolytischen Trog vom Versuchsaufbau, wie er bei Aubrecht u. a. (2006) beschrieben wird, unterscheidet. Aus messtechnischen Gründen, wurde die Elektrode für das tieferliegende Potenzial (lo) nicht an das Kupferkabel gekoppelt, sondern stellt die (erste) Bezugselektrode in der Nährlösung dar. Die Ergebnisse der Messung sind aber dennoch vergleichbar. Beachtet werden muss außerdem, dass immer wieder von der durchwurzelten Fläche eine Baumes gesprochen wird. Sowohl die Kupferscheibe, als auch die Wurzeln stellen jedoch korrekterweise dreidimensionale Objekte dar. Neben der Abschätzung der Wurzeloberfläche (AZ) soll mit Hilfe dieses Verfahrens auch die Grenze des durchwurzelten Raumes („Baumscheibe“) abgeschätzt werden.

Die modellhafte Betrachtung mit idealen Leitern zeigt, dass Messobjekte (bis ca. $2 \mathrm{~cm}$ Tiefe) verlässlich erfasst werden können. Setzt man das Modell hinsichtlich seiner Dimension mit reellen, baumspezifischen Größen ins Verhältnis (bspw.: $1 \mathrm{~cm} \equiv 100 \mathrm{~cm}$ ), so kann vermutet werden, dass die Grenze einer $8 \mathrm{~m}$ breiten und bis $2 \mathrm{~m}$ tiefen durchwurzelte „Fläche“ eines Baumes mit diesem Verfahren sondiert werden kann. Die Tiefe der absorbierenden Feinwurzeln von $2 \mathrm{~m}$ ist dabei eine praxistaugliche Größe. Allerdings sei auch an dieser Stelle darauf hingewiesen, dass zwischen dem Kupferkabel (elektrische Leitfähigkeit: $58 \times 10^{6} \mathrm{~S} \mathrm{~m}^{-1}$ ) und der hier verwendeten Kochsalzlösung (elektrische Leitfähigkeit: $3,7 \mathrm{~S} \mathrm{~cm}^{-1}$ ) erhebliche Leifähigkeitsunterschiede und damit hohe Leitfähigkeitskontraste herrschen. Solch große Leitfähigkeitskontraste sind zwischen Wurzel und Substrat in der Regel nicht gegeben, so dass sich die Grenze der Wurzeln („Baumscheibe“) elektrisch deutlich schwächer abzeichnen sollte.

Auch geht man im hier angesprochenen Modell von einer Kupferscheibe als homogen durchwurzelte Fläche im Halbraum aus. Dieses ideale Bild ist praxisfern, denn der Verlauf von Wurzeln wird bspw. durch die Konkurrenz von Wurzeln benachbarter Bäume beeinflusst, bzw. von der Verfügbarkeit von Wasser und Nährstoffen (Streckenbach (2009); Yanai u.a. (2006)). Daraus ergibt sich die Tatsache, dass auch die Absorptionsflächen der Wurzeln ungleichmäßig verteilt sind. Zudem enden Feinwurzeln nicht abrupt an einer bestimmten Stelle in der Peripherie des Stammes, wie sie die Kupferscheibe fälschlicherweise modelliert.

Die weiteren Untersuchungen machen auch deutlich, dass eine Art Äquivalenzprinzip, wie es beispielsweise von geoelektrischen Sondierungen bekannt ist, auch hier zu beobachten ist (Lange (2005)). So können Objekte mit einem relativ hohen elektrischen Widerstand in geringerer 


\section{Diskussion}

Tiefe ähnliche Ergebnisse liefern, wie deutlich tieferliegende Objekte mit deutlich höherer Leitfähigkeit. Dieser Effekt ist besonders beim Vergleich der zwei unterschiedlich dimensionierten Kupferscheiben in verschiedenen Tiefen zu beobachten (siehe Abbildung 4.8). Für die Zielstellung, der Abschätzung der Wurzeloberfläche, ist dieses Phänomen äußert kritisch zu bewerten, denn in den bereits beschriebenen Arbeiten (Aubrecht u. a. (2006) sowie Čermák u. a. (2006)) ist weder die Tiefe der Objekte (Wurzeln) bekannt, noch die Oberfläche der Wurzeln. Letztere soll gerade durch diese Messung abgeschätzt werden.

Reelle Messungen im Gelände unter nahezu gleichen Bedingungen zeigten drei verschiedene Bereiche einer jeden Messlinie (siehe Abbildung 4.9). Die dort beschriebenen einzelnen Messlinien des Baumes sind jedoch lediglich parallelverschoben und unterscheiden sich nur hinsichtlich ihrer absoluten Werte. Damit unterscheiden sich die Kurvenverläufe deutlich von denen, die von Čermák u. a. (2006) angegeben wurden. Das Auftreten von relativ betrachtet ähnlichen Kurvenverläufen konnte auch bei anderen Messobjekten (zwei Fichten auf den Schillerwiesen bei Göttingen) bestätigt werden. Die Unterscheidung der durchwurzelten Fläche von der außerhalb liegenden Fläche ist anhand der Kurve nicht möglich. Auch anhand eines Datensatzes von Čermák konnte die in Aubrecht u. a. (2006) beschriebene „Potentialcharakteristik“ nicht gefunden werden. Auch nach schriftlicher Nachfrage konnte das Problem nicht gelöst werden. Die Abgrenzung des durchwurzelten Bereiches erscheint eher subjektiv und dadurch fragwürdig. Derzeit wird an einer modifizierten Methode, der modified earth impedance method (MEI), gearbeitet (Čermák u. a. (2013)), bei der versucht wird, diese und anderen Einflussfaktoren von dem entscheidenden Wert zu separieren.

Eine Innovation der Bodenwiderstandsmethode ist die in den genannten Arbeiten beschriebene Formel (Gleichung (2.3)). Eine wesentliche Größe ist die Spannungsdifferenz zwischen den Messpolen M und N, die nach dem Ohmschen Gesetz in den Erdungswiderstand umgerechnet werden kann (vgl. Abschnitt 2.3 bzw. Abschnitt 2.4). Der Versuch der räumlichen Betrachtung wird über die „wandernde“ Elektrode N ermöglicht, also über Erdungswiderstände in unterschiedlicher Entfernung zum Baum. In der angesprochenen Formel ist außerdem der spezifische elektrische Widerstand des Holzgewebes verankert. Dieser soll durch eine separate Messung am Stamm ermittelt werden. Dieser Gedankengang erscheint angesichts der in Abschnitt 5.1 angestellten Überlegungen sinnvoll. Die Messungen zur Ermittlung des elektrischen Widerstandes des Holzgewebes wird allerdings durch eine Vierpunktmessung direkt am Stamm durchgeführt. Aufgrund des deutlich unterschiedlichen Versuchsdesigns zur Erdungsmessung, erscheint das Einfließen eines derartig ermittelten elektrischen Widerstandes am Stamm nicht sinnvoll.

Es ist fraglich, ob der spezifische elektrische Widerstand von "Stammholz" mit „Wurzelholz" gleichzusetzen ist. Hierfür werden in den genannten Publikationen keine Angaben gemacht. Wie bereits erwähnt, unterscheiden sich diese Gewebetypen voneinander (Abschnitt 2.1.1).

Insgesamt muss weiterhin hinterfragt werden, ob diese Methode einen Mehrgewinn für die Problemstellung bedeutet. Letztendlich werden hier, wie auch bei der Erdungsmessung, Spannungsdifferenzen bei niedrigen Frequenzen gemessen. Die nahezu gleichstromartigen Verhältnisse und die damit verbundenen Schwierigkeiten bleiben bestehen, siehe auch Abschnitt 5.1 . Die Erkenntnisse der Erdungsmessungen im Gelände können so auch bei diesem Verfahren 
hilfreich sein, denn bei den genannten Veröffentlichungen wird weder die Temperatur, noch die Leitfähigkeit des Bodens mit einbezogen. Es konnte gezeigt werden, dass Temperatur resp. Bodenfeuchte und Erdungswiderstand linear miteinander korrellieren (siehe Abbildung 4.3). Temperatur und Bodenfeuchte werden in der von Aubrecht u.a. (2006) bzw. Čermák u.a. (2006) erarbeiteten Formel weder mit einbezogen, noch wird auf eine Möglichkeit der Korrektur hingewiesen. Die Zunahme der Temperatur und die Zunahme der Bodenfeuchte würde gemäß Gleichung (2.3) eine höhere Absorptionsfläche $(S)$ bedeuten. Wenn sich also die Temperatur und die Bodenfeuchte verändern, ändert sich auch rechnerisch die Absorptionsfläche, obwohl diese faktisch konstant bleibt. Da der spezifische elektrische Widerstand der Leitgewebe eines Baumes $(\rho)$ ebenfalls temperaturabhängig ist und als weitere Größe in die bereits genannte Formel eingeht, ergibt sich hier ein weiterer sensibler Parameter und damit eine weitere Schwachstelle der hier diskutierten Ergebnisse. Die hier angeführten Versuche und Überlegungen zeigen, dass bei den Veröffentlichungen von Aubrecht u. a. (2006) bzw. Čermák u. a. (2006) sowohl die Messungen als solche, als auch die darauffolgenden Berechnungen skeptisch betrachtet werden müssen. Die Bodenwiderstandsmethode ist in der beschriebenen Form unter reellen Bedingungen weder geeignet Feinwurzeln zu quantfizieren, noch die durchwurzelte Fläche von Bäumen zu ermitteln.

\subsection{Impedanzspektroskopie}

Bereits in den Arbeiten von Cao u. a. (2011), Ozier-Lafontaine und Bajazet (2005) wird deutlich, dass die frequenzabhängige Betrachtung des elektrischen Widerstandes neue Möglichkeiten für genauere biologische Untersuchungen offenbart.

Im Gegensatz zu den monofrequenten Verfahren, lassen sich bei kapazitiven Messverfahren und der Impedanzspektroskopie Kapazitäten ermitteln. Zeigt sich Wurzelwachstum durch Anstieg von Kapazitäten, lässt sich dieses deutlich einfacher anhand der physikalischen Messwerte verfolgen, denn Kapazitäten addieren sich einfach auf, ohmsche Widerstände hingegen addieren sich mit ihrem Kehrwert.

Die Impedanzspektroskopie mit nachfolgender Invertierung hat aber auch aus weiteren Gründen einen Mehrwert gegenüber den monofrequenten Methoden. Ersatzschaltbilder, die als Modell für die Versuchsanordnung eines Stecklings in hydroponischem Medium bzw. einer Pflanze in Nährboden dienen, zeigen, dass das jeweilige Ersatzschaltbild jedoch komplex ist. Zahlreiche Parameter nehmen in unterschiedlicher Stärke einen Einfluss auf das Impedanzspektrum. Die Modelle und die Schlussfolgerungen daraus werden hier im Zusammenhang mit den beiden Hauptversuchen diskutiert. Mit diesen wurden erstmalig echte Zeitreihen des Wurzelwachstums generiert. Diese Zeitreihen können durch die hier angewendete zerstörungsfreie fotografische Auswertung mit biologischen Werten verglichen werden.

Zunächst sollen jedoch die Versuchsbedingungen für die impedanzspektroskopischen Messungen genauer ausgeführt werden. Nicht zuletzt durch die Beobachtung der Impedanz über z. T. mehrere Wochen und der damit verbundenen hohen Datendichte, können wesentliche Schlussfolgerungen für die optimale Messmethodik aufgedeckt werden. 


\section{Diskussion}

Betrachtet man grundsätzlich die Rohdaten der gemessenen Untersuchungsobjekte und insbesondere den negativen Phasenwinkel, so ist bereits an dieser Stelle klar, dass es vielfach zu einer negativen Phasenverschiebung kommt und damit hauptsächlich Kapazitäten das Modell des Stecklings in dem hier beschriebenen Versuchsaufbau prägen. In einigen Fällen treten positive Phasenwinkel auf. Diese scheinbare Induktivität bei kleinen Frequenzen $(<3 \mathrm{~Hz})$ ist dabei recht niedrig (Verschiebung des Phasenwinkels meistens $<2^{\circ}$ ). Phasenverschiebungen und die damit verbundenen erhöhten Imaginärteile sollten hier aufgrund der sehr niedrigen Frequenz keine Rolle spielen. Bei sehr hohen Frequenzen (>500 kHz) ist der Phasenwinkel sehr hoch, allerdings sind hier deutliche Schwankungen zu erkennen. Teilweise wechselt das Vorzeichen mehrmals bei aufeinanderfolgenden Frequenzen. Die gerade bei sehr niedrigen bzw. sehr hohen Frequenzen auftauchenden positive Phasenwinkel und damit auch der positiven Imaginärteile, können als Messfehler des Frequenzganganalysators (Solartron 1260) gedeutet werden. Diese treten auf, obwohl bei sämtlichen Messungen mit geschirmten Leitungen gearbeitet wurde. Streukapazitäten und Selbstinduktivitäten sind häufige Ursachen für Messfehler bei höheren Frequenzen (Ende und Mangold (1993)).

Einfache frequenzabhängige Widerstandsmessungen mit der in Abbildung 3.5 gezeigten Testschaltung haben deutlich gemacht, dass den Messdaten, abgesehen von den positiven Phasenwinkeln, grundsätzlich vertraut werden kann. Die Testschaltungen, die hinsichtlich der absoluten einzelnen Wechselstromwiderstände in sehr ähnlichen Größenordnungen lagen (Kapazitäten und ohmsche Widerstände wurden den Werten nach Invertierung der Daten aus Cao u. a. (2011) angeglichen bzw. als reelles Ersatzschaltbild „nachgebaut“), ergaben Abweichung von ca. $2 \%$, in wenigen Fällen bis $7 \%$. Die größeren Abweichungen wurden beim Imaginärteil der Impedanz beobachtet und lassen darauf schließen, dass das Impedanzspektrum vorwiegend durch Kapazitäten im Messsystem durch die Koaxialkabel, Koaxialverbinder und Multiplexer beeinflusst wird. Da das Versuchsdesign, insbesondere die Kabellängen, für alle Stecklinge eines jeden Versuches gleich blieben, können die Abweichungen der in dieser Arbeit erhobenen Messdaten von den wahren Werten als systematische Fehler behandelt werden.

\subsubsection{Vorversuche zur Untersuchung der Randbedingungen}

Für die Kontaktierung bzw. die optimale Wahl und Anordnung besonders der Stecklingselektroden herrscht eine gewisse Uneinigkeit in der Literatur. Während beispielsweise bei OzierLafontaine und Bajazet (2005) Silber-Silberchlorid-Elektroden Verwendung finden, werden bei Cao (2011) Silberelektroden benutzt. Sogar unterschiedliches Elektrodenmaterial für die Elektrode am Spross und im Substrat (Platin- und Silberelektroden) finden sich in einer Messanordnung bei Repo u.a. (2005). Die hier durchgeführten Tests zeigen, dass einfache vernickelte Stecknadeln, wie zu erwarten, nicht geeignet sind. Hier traten bereits nach wenigen Tagen optisch erkennbare Korrosionsstellen auf. Die hier verwendeten Edelstahlelektroden (Kanülen) zeigen unter dem Rasterelektrodenmikroskop visuell keine und chemisch betrachtet nur geringe Veränderungen. Eine erhöhte Kohlenstoffkonzentration wurde bei dem Teil der Nadel festgestellt, der im Steckling steckte. Diese wurde mit dem Anhaften von Zellen des Stecklings auf der Elektrodenoberfläche erklärt. Bei dem Teil der Nadel, die sich außerhalb des Steck- 
lings befand, fällt die erhöhte Konzentration von Sauerstoff auf, die möglicherweise mit dem direkten Kontakt dieses Nadelstücks zur Umgebungsluft erklärbar ist.

Insgesamt hat sich gezeigt, dass das verwendete Elektrodenmaterial beim direkten Vergleich von Silber- und Edelstahlelektroden weniger ausschlaggebend für die Messung ist, als die Anordnung und die Anzahl dieser Elektroden. Nahezu gleiche Ergebnisse wurden bei der Berücksichtigung der gleichen Messanordnung, aber anderen Elektrodenmaterialien erzielt. Hier wurden lediglich kleine Abweichungen der jeweiligen Impedanzspektren entdeckt.

Diese Differenz ist auf Messfehler zurückzuführen, da es messtechnisch nicht möglich ist, die beiden zu vergleichenden Elektroden exakt an gleicher Stelle zu platzieren. Kleinste Abweichungen der Anordnung führen dabei zu deutlichen Fehlern. Bereits hier wird die Anfälligkeit der Messmethodik auf externe Faktoren deutlich.

Vierpunkt- und Zweipunktmessung liefern unterschiedliche Ergebnisse (siehe Abbildung 4.13 bzw. Abbildung 4.14). Die Dreipunktmessungen müssen an dieser Stelle differenziert betrachtet werden. Hier stellt sich heraus, dass z. B. die Vierpunktmessungen B und die Dreipunktmessungen D vergleichbare, die Dreipunktmessungen $\mathrm{C}$ und $\mathrm{E}$ jedoch deutlich andere Ergebnisse liefern. Die deutlichste Abweichung liegt dabei in den niedrigen Frequenzen, besonders beim Imaginärteil der Impedanz, und lässt sich mit einem sogenannten Polarisationseffekt erklären. Im Falle der gekoppelten Mess- und Speiseelektrode (Dreipunktmessung C) mit einer sehr geringen kontaktierten Mantelfläche von wenigen $\mathrm{mm}^{2}$ ist die Stromdichte um ein vielfaches höher als beim Edelstahlblech als kombinierte Mess- und Speiseelektrode am Grund des Becherglases. Die Auswirkung der vergrößerten Elektrodenoberfläche zeigt sich besonders beim Vergleich der Dreipunktmessungen D und E. Bei diesen beiden Messungen unterscheidet sich lediglich die Oberfläche der gekoppelten Elektrode in der Nährlösung. Dabei ist die Elektrode bei der Messung D größer als bei Messung E. Die veränderte Oberfläche macht sich im niederfrequenten Bereich des Impedanzspektrums bemerkbar (bis ca. $100 \mathrm{~Hz}$ ).

Polarisationseffekte treten bei Gleichstrommessungen (DC) auf, da sich hier die Richtung des elektrischen Stromes nicht ändert und es zu einer „Aufladung“ durch Ladungstrennung kommt. Bei den o.g. Dreipunktmessungen zeigt sich der Polarisationseffekt daher bei den niedrigen, „gleichstromartigen“ Frequenzbereichen von nur wenigen Hz. Bei multifrequenten Untersuchungen mit Nährlösungen wurde festgestellt, dass die Stärke des Polarisationseffekte stark vom Elektrodenmaterial und der Leitfähigkeit der Nährlösung abhängt (Davey und Kell (1998a)). Weiterhin wird der Versuch beschrieben, diesem Effekt modellhaft zu begegnen (Davey und Kell (1998b)), so dass dieser separat von den relevanten Impedanzelementen betrachtet werden kann. Polarisationseffekte treten dabei nicht nur bei Zweipunktmessverfahren, sondern auch bei Vierpunktmessungen unter bestimmten Umständen auf. In der vorliegenden Arbeit traten Polarisationseffekte lediglich bei den Zweipunktmessungen auf. Die Vierpunktmessungen zeigten keinerlei Auffälligkeiten bei niedrigen Frequenzen. Die modellhafte Berücksichtigung dieses Polarisationseffektes durch zusätzliche Impedanzelemente im Ersatzschaltbild wurde in dieser Arbeit nicht angestrebt.

Bei weiterer Betrachtung des Versuchs ist auch der Einfluss von Übergangswiderständen zu erkennen. Während die Messwerte der Dreipunktessungen C in ähnlicher Größenordnung wie 


\section{Diskussion}

die der Zweipunktmessungen A liegen, erscheint Dreipunktmessung E deutlich niederohmiger und erreicht ähnliche Größenordnungen wie die der Vierpunktmessungen B. Daraus lässt sich schlussfolgern, dass die Übergangswiderstände zwischen Elektrode und Steckling deutlich stärker in Erscheinung treten, als zwischen Elektrode und Nährlösung. Übergangswiderstände zeigen sich dabei vorwiegend im Realteil der Impedanz. Bei der Umsetzung des Versuchsdesigns muss bei Zwei- und Dreipunktmessungen auf eine ausreichende Fläche der Elektrode in der Nährlösung geachtet werden, da eine größere Kontaktfläche den Übergangswiderstand verringert. Eine Kopplung von Mess- und Speisekreis auf der Seite der Stecklingselektrode sollte nur dann erfolgen, wenn mit unpolarisierbaren Elektroden gearbeitet wird. Bei der impedanzspektroskopischen Erhebung von Daten in flüssigen Medien gilt die Vierpunktmessung als genaueste Messmethode (Hofmann (2009)).

Die Untersuchungen mit verschieden leitfähigen Nährlösungen zeigen, dass besonders die Nährlösung mit $54 \mu \mathrm{S} \mathrm{cm}^{-1}$ durch einen deutlich höheren Realteil gegenüber den beiden anderen untersuchten Nährlösungen auffällt (vgl. Abbildung 4.15). Der Imaginärteil zeigt in der Tendenz ein ähnliches Bild, jedoch in geringerer Deutlichkeit. Bei den Vergleichen zwischen Nährlösungen mit höherer Leitfähigkeit (zwischen $254 \mu \mathrm{S} \mathrm{cm}^{-1}$ und $508 \mathrm{\mu S} \mathrm{cm}^{-1}$ ) ist die Auswirkung auf die Gesamtimpedanz überraschend gering. Die Leitfähigkeit der Nährlösung bei sonst gleichen Bedingungen spielt folglich hauptsächlich im unteren Leitfähigkeitsbereich eine bedeutende Rolle.

Dies lässt vermuten, dass der Zusammenhang zwischen dem Realteil der Impedanz und der Leitfähigkeit der Nährlösung nicht linear ist. Für künftige Versuche erscheint es zweckmäßig, die Leitfähigkeit der Nährlösung möglichst groß zu wählen. Da vermutet wird, dass sich die Nährlösung nach der Modellierung als ohmscher Widerstand im Ersatzschaltbild darstellt (siehe Abschnitt 5.3.2), ist es wichtig, diesen Faktor schon bei der Versuchsplanung zu berücksichtigen.

Die Eintauchtiefe oder die mit Nährlösung benetzte Fläche des Stecklings spielt ebenfalls eine zentrale Rolle. Bei den Untersuchungen der Randbedingungen konnte gezeigt werden, dass eine geringe Veränderung des Wasserstandes einen massiven Effekt auf den Realteil der Impedanz hat. Bei den beiden Hauptversuchen ist dies im Versuchsdesign durch Verwendung der Mariottesche Flasche berücksichtigt worden. Jedoch zeigen sich auch im Verlauf von Real- und Imaginärteil der Impedanz Schwankungen, die durch Wurzelwachstum oder Temperatureffekte nicht erklärt werden können. Die Vermutung, dass sich die Schwankungen des Realteils mit fotosynthetischen Prozessen erklären, kann ausgeschlossen werden, da diese Schwankungen auch bei nicht belaubten und bewurzelten Stecklingen auftreten. Eine Erklärung für diese Schwankungen könnten kleinste Veränderungen des Wasserstandes sein, die zu beobachten waren, obwohl die Mariottesche Flasche diesen im Versuchsaufbau egalisieren sollte. Vorraussetzung für das Überheben der Nährlösung von der einen auf die andere Seite ist ein Druckunterschied. Bei Verbrauch von Wasser durch die Pflanzen und/oder Verdunstung muss es jedoch zu divergierenden Wasserpegeln zwischen Wasserreservoir und Küvette kommen. Um diesen nicht auszuschließenden Effekt einzuschätzen, sollen hier einige Überlegungen angestellt werden.

Bevor Nährlösung vom Reservoir in die Küvette fließt, bildet sich am unteren Ende des 
Röhrchens a (siehe dazu Abbildung 3.10 eine Luftblase, die langsam an Volumen zunimmt und sich alsbald von der Röhrchenspitze ablöst. Anschließend bilden sich neue Luftblasen, erst danach kann Wasser übergehoben werden. Schätzt man das Volumen einer Luftblase, als kleinste Volumeneinheit, die den Wasserstand verändern vermag, so kann diese Höhenänderung näherungsweise bestimmt werden. Angenommen werden soll hier eine Blasengröße von $6 \mathrm{~mm}$ Durchmesser. Daraus ergibt sich ein Blasenvolumen von ca. $113 \mathrm{~mm}^{3}$. Das Volumen einer Blase bewirkt somit eine Höhenänderung von 0,0098 mm bei der Küvette aus Hauptversuch $1 \mathrm{~cm}$ bzw. 0,006 $47 \mathrm{~mm}$ bei der Küvette aus Hauptversuch 2. Dieser Anstieg des Wasserstandes in der jeweiligen Küvette ist so gering, dass sich dies im Impedanzspekrum kaum zeigen würde. Selbst bei einem Überfließen von bspw. 10 Blasen, wäre der Effekt zu gering. Diese Tatsache bedeutet aber auch, dass die Mariottesche Flasche eine geeignete Einrichtung für die hier durchgeführten Versuche darstellt.

Da bei der Impedanzspektroskopie frequenzabhängig Widerstände gemessen werden, können auch diese Messwerte durch Temperaturschwankungen beeinflusst werden. Die periodischen Schwankungen des Realteils von Tag- und Nachtwerten unterscheiden sich je nach Frequenz zum Teil erheblich und signifikant (Schwankungen bis zu 14\%). Speziell für elektrische Widerstandsmessungen an Holz beobachtet Du (1991) ebenso eine Abnahme des elektrischen Widerstandes bei steigender Temperatur. Dabei wird festgestellt, dass diese zu den Haupteinflussfaktoren gehört. Die Streuung der Impedanzmesswerte ist bei den Tagwerten deutlich erhöht. Möglicherweise ist diese Streuung unter Einfluss des Lichtes durch eine höhere pflanzenphysiologische Aktivität außer Fotosynthese (s. o.) zu deuten.

In der vorliegenden Arbeit stellt sich heraus, dass die Dimension des Stecklings direkte Auswirkung auf das Impedanzspektrum hat. Es konnte gezeigt werden, dass es einen annähernd linearen negativen Zusammenhang zwischen dem Durchmesser des Stecklings und dem Realteil resp. dem Imaginärteil der Impedanz bei ausgewählten Frequenzen gibt. Diese Beobachtung konnte bereits bei den monofrequenten Messungen gemacht werden (siehe Abschnitt 4.1.3). Während bei den impedanzspektroskopischen Untersuchungen ein linearer Trend vorzufinden war, beschreibt die Ausgleichsfunktion bei den Erdungsmessungen eine Exponentialfunktion. Die unterschiedliche Geometrie der Stecklinge (größere Querschnitte) führt offenbar zu deutlich höheren Leitfähigkeiten. Dieser Befund kann mit der Addition von Widerständen und Kapazitäten aufgrund größerer Zellverbände beschrieben werden. In der Summe weisen dickere Stecklinge über den gesamten Frequenzbereich deutlich niederohmigere Werte auf als dünnere.

Um sich dem Einfluss des Stecklings weiter zu nähern, wurden die Modellparameter aus Cao u.a. (2011) für einen bewurzelten Steckling aufgegriffen und „vorwärtsgerechnet“. Mit Hilfe des in Abschnitt 3.4 genannten Programmes Impedanz können aus den Modellparametern reale Impedanzspektren erzeugt werden. Aus der genannten Publikation geht hervor, dass die Leitfähigkeit des Stecklings mit dem ersten solitär stehenden RC-Glied modelliert werden kann und u.a. die Werte der Kapazitäten gut mit dem Wurzelwachstum korrelieren (vgl. Abbildung 2.8b.

Auf diese Weise kann ein Szenario erstellt werden, bei dem der Einfluss der Leitgewebe 

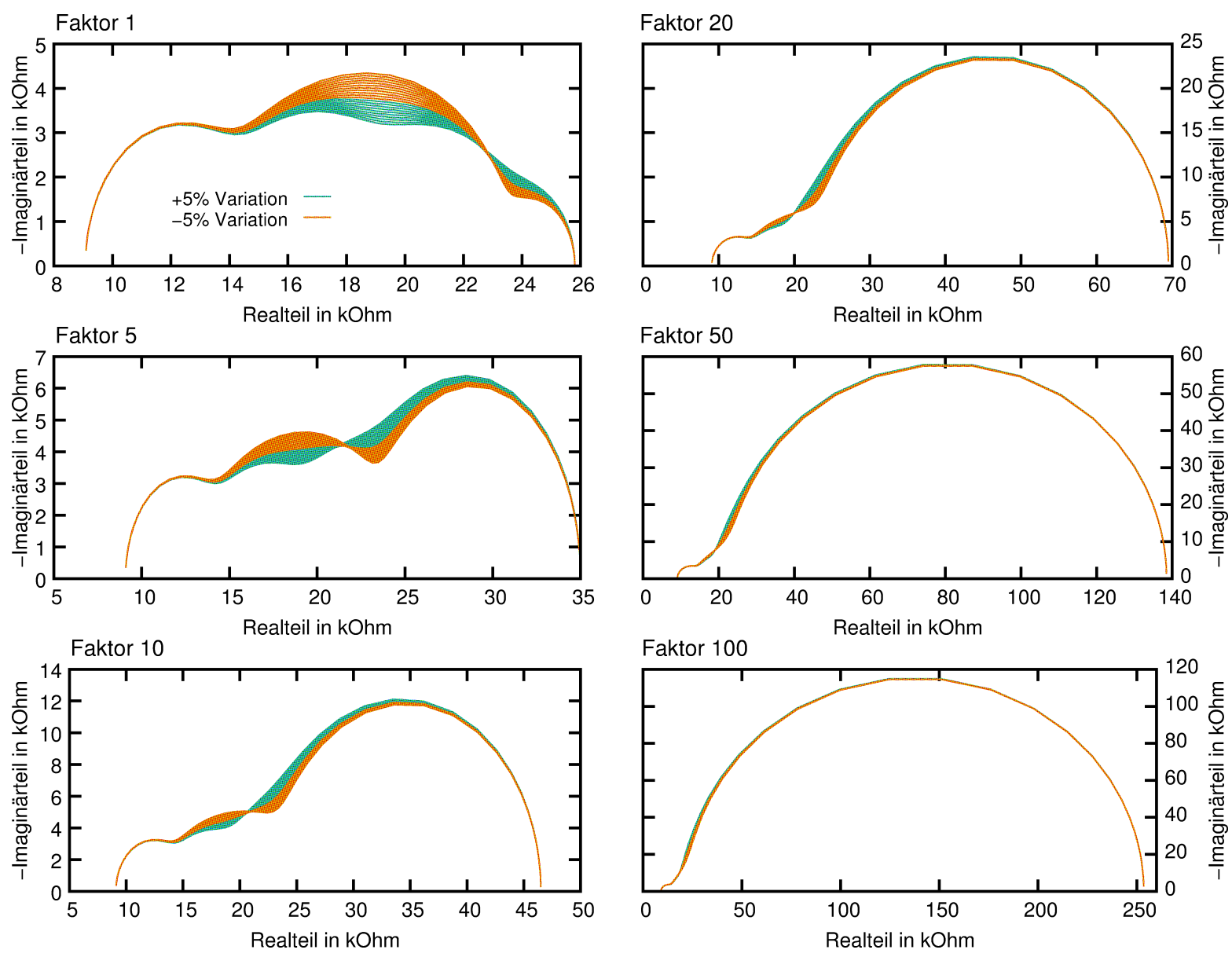

Abbildung 5.1: Vorwärtsrechnung bei Variation einer Kapazität bei sukzessiv ansteigendem $\tau$. Weitere Erläuterungen finden sich im Text.

des Stecklings auf das Gesamtspektrum schrittweise erhöht wird. Modellhaft geschieht dies durch die schrittweise Erhöhung der Kapazität (C1) und des ohmschen Widerstandes (R1). Messtechnisch kann diese Erhöhung durch eine Abnahme des Durchmessers des Stecklings bzw. durch das schrittweise vertikale Versetzen der Messelektrode in Richtung der oberen Schnittfläche des Stecklings erfolgen. Bei jeder dieser schrittweisen Erhöhungen kann eine Zu- oder Abnahme der Wurzeloberfläche modelliert werden, indem die Werte der für das Wurzelwachstum identifizierten Impedanzelemente (hier: Kapazitäten) erhöht (Zunahme des Wurzelwachstums) bzw. verringert werden (Abnahme des Wurzelwachstums). In Abbildung 5.1 ist ein solcher Variationslauf dargestellt.

Hier wird die Zeitkonstante $(\tau)$, die sich aus dem Produkt von R1 und C1 errechnet (siehe Abbildung 2.8b), sukzessive von Faktor 1 auf 100 erhöht. In jeder Einzelgrafik variiert die Kapazität (hier: C3) jeweils um $\pm 5 \%$. Dabei bedeutet eine Zunahme der Kapazität (+) die Erhöhung der Wurzelmasse und die Abnahme der Kapazität (-) eine Abnahme derselben. Während die Variation der Kapazität bei Faktor 1 deutlich durch eine Zunahme bzw. Abnahme des Imaginärteils erkennbar ist, nimmt dieser Effekt bei Zunahme der Werte des RC-Gliedes sukzessive ab. Ab einem 50fach erhöhten Wert von $\tau$, ist die Variation der Kapazität kaum anhand der Grafik auszumachen. 
Eine Zunahme der Werte des RC-Gliedes hat somit zur Folge, dass die Größen anderer im Modell (Ersatzschaltbild) befindlichen Elemente in den Hintergrund treten („Maskierungseffekt"). Will man folglich das Wurzelwachstum bzw. andere Größen dieser Messanordnung untersuchen und quantifizieren, so müssen die anderen Modellparameter deutlich geringer sein. Für die Planung des Versuchsdesigns sollten Messungen an dimensionsstärkeren Stecklingen durchgeführt werden. Die Messelektrode sollte dabei möglichst nah an der Nährlösungsoberfläche platziert werden, ohne dabei in Kontakt mit der Flüssigkeit zu kommen.

Auch wurde untersucht, ob der verholzte Teil des Stecklings als solcher einen Einfluss auf das Impedanzspektrum hat. Offenbar wirkt der durchströmte Bereich von Xylem und Phloem in Längsrichtung stark hochohmig. $50 \mathrm{~mm}$ des verholzten Stecklingsgewebes macht hier je nach Frequenz und Durchmesser des Stecklings bis zu $50 \%$ des gesamten Realteils der Impedanz aus.

Insgesamt lassen diese Ergebnisse die Aussage zu, dass ohne die Beachtung der genannten Einflussfaktoren, die im Rahmen der Vorversuche gefunden wurden, Daten nicht miteinander verglichen werden können. Bei Weiterverfolgung der Impedanzspektroskopie sollten künftig Standardbedingungen geschaffen und eingehalten werden (Messtechnik, Elektrodenanordnung, Elektrodenmaterial, Temperatur). Kendall u. a. (1982) weisen bereits auf den Einfluss der bodenspezifischen Faktoren hin. Bei Kendall u. a. konnte gezeigt werden, dass es selbst unter Laborbedingungen mit scheinbar konstanten externen Rahmenbedingungen zu einer Beeinflussung kommt.

\subsubsection{Hauptversuche}

\section{Allgemeine Betrachtung der physikalischen und biologischen Daten}

Sowohl im Hauptversuch 1, als auch im Hauptversuch 2 kann das Wurzelwachstum nicht nur visuell über die Versuchszeiträume, sondern auch quantitativ mit Hilfe der fotografischen Auswertung verfolgt werden. Diese innovative Art der Quantifizierung des Wurzelwachstums erfolgt zerstörungsfrei und generiert dabei echte Zeitreihen des Wurzelwachstums eines jeden Stecklings bei gleichzeitiger impedanzspektroskopischer Messung. Die so erhobenen Daten zur Wurzellänge und Wurzeloberfläche sind allerdings fehlerbehaftet. Gerade bei der Ausbildung der Wurzeln zweiter Ordnung bspw. bei Hauptversuch 1, bzw. beim Nachlassen des Wurzelwachstums in Hauptversuch 2, ist eine gewisse Streuung erkennbar (Abbildung 4.23 sowie Abbildung 4.25. Diese muss mit Messfehlern bei der Auswertung der Fotos mit WinRHIZO® erklärt werden.

Bei der Versuchsplanung wurde besonders auf die geringe Tiefe der Küvetten geachtet, um eine seitliche Ausbreitung der Wurzeln zu erzwingen. Auf diese Weise konnte der Großteil der Wurzeln fotografisch gut erfasst werden. Allerdings gab es besonders beim Hauptversuch 2 Wurzeln, die durch den Steckling selbst verdeckt wurden, da sich diese rückseitig z. T. vertikal entlang des Stecklings ausbreiteten. So konnten diese Wurzeln fotografisch nicht erfasst werden. Auch bei der vorderseitigen Ausbreitung der Wurzeln in vertikaler Richtung war eine Erfassung der Wurzeln nicht möglich, da hier der Kontrast der Wurzeln zum Steckling im 


\section{Diskussion}

Hintergrund nicht ausreichte, um die Wurzel zu erfassen. Diese Fehler werden als systematische Fehler eingeschätzt, so dass sie bei Weiterverwendung der Daten keinen Einfluss auf das Gesamtergebnis haben. Die meisten Fehler ergaben sich jedoch bei beiden Versuchen gegen Ende des jeweiligen Versuchszeitraumes.

Eine mögliche Fehlerquelle bei Hauptversuch 1 geht mit der Ausbildung der Feinwurzeln zweiter Ordnung einher. Die juvenilen Feinwurzeln weisen z. T. sehr geringe Durchmesser auf und verjüngen sich zusätzlich an der Basis, so dass hier in manchen Fällen nur Teilabschnitte dieser Wurzeln von dem Auswerteprogramm erkannt wurden.

Bei beiden Versuchen kann die geringe Streuung der Werte besonders gegen Ende des Versuchszeitraumes mit dem teils geringen Wachstum von einem zum anderen Tag erklärt werden. So führen kleine Veränderungen der Belichtung oder des Kamerastandortes zu leichten Abweichungen der gemessenen Parameter. Insgesamt können die Abweichungen jedoch als gering bewertet werden. Bei Betrachtung der Wurzellänge gegen die Wurzeloberfläche ist anfänglich eine Abweichung des im weiteren Verlauf gut passenden linearen Modells zu erkennen. Im Bereich der niedrigen Werte nimmt das Oberflächenwachstum zunächst deutlicher zu, als die Wurzellänge. Bei der Berechnung der Oberfläche mit Hilfe des Programmes WinRHIZO® geht hier neben der Wurzellänge der Durchmesser der Wurzeln ein, um daraus die Gesamtoberfläche (surface) zu berechnen. Folglich ändert sich der Durchmesser der Wurzeln zu Beginn stärker, so dass die Wurzeloberfläche insgesamt stärker als die Wurzellänge zunimmt. Der Effekt kann also mit dem Dickenwachstum der Wurzeln erklärt werden. Insgesamt scheint das hier vorgestellte fotografische Verfahren zur Wurzelquantifizierung sehr gut geeignet.

Der Verlauf von Real- und Imaginärteil bei verschiedenen Frequenzen zeigt, neben den bereits diskutierten Temperaturschwankungen (Tagesgänge), im Zeitraum des Wurzelwachstums keinen eindeutigen Trend. Es wird vermutet, dass sich das Längenwachstum der Wurzeln dabei besonders durch abnehmende Realteile bemerkbar macht (Zunahme der Kapazität und Abnahme des elektrischen Widerstandes).

Bei der Untersuchung der Rohdaten (Realteil resp. Imaginärteil der Impedanz und der Wurzellänge) zeigen sich zum Teil sehr deutliche Zusammenhänge, sowohl bei Hauptversuch 1, als auch bei Hauptversuch 2. Diese z. T. starken Zusammenhänge müssen jedoch differenziert betrachtet werden.

Betrachtet man die Zusammenhänge zwischen Wurzellänge und Imaginärteil resp. Realteil der Impedanz, so fällt auf, dass sich diese bei verschiedenen Frequenzen unterscheiden. Während bei einigen Frequenzen ein bestimmtes Muster des Verlaufs der Zusammenhänge erkennbar ist, ist dieses Muster bei anderen Frequenzen gegensätzlich. Zwar ändert sich mit wechselnder Frequenz der Phasenwinkel und damit der kapazitive Anteil der Impedanz, jedoch bleibt offen, warum sich der Zusammenhang umkehrt.

Vergleicht man die Zusammenhänge der genannten Parameter verschiedener Stecklinge eines Versuches bei gleicher Frequenz, so erkennt man auch hier teilweise gegensätzliche Verläufe. Dieser Befund verwundert, da alle Stecklinge eines jeden Versuches gleichen Versuchsbedingungen ausgesetzt sind. Lediglich die Dicke der Stecklinge und die Höhe der Einstichstelle der Elektroden können sich um wenige mm voneinander unterscheiden. 
Wie bereits erwähnt, ist sowohl bei Hauptversuch 1, als auch bei Hauptversuch 2 in Dreipunktmessung gemessen worden. Es konnte vorab gezeigt werden, dass das Elektrodenmaterial keinen entscheidenden Einfluss auf das Impedanzspektrum hat. Die Dreipunktmessungen aus beiden Hauptversuchen müssten demnach ähnliche Ergebnisse liefern. Die Bestimmtheitsmaße liegen teilweise in ähnlichen Größenordnungen, so dass zumindest die Güte der Zusammenhänge recht ähnlich erscheint. Die Zusammenhänge zwischen Realteil der Impedanz resp. Imaginärteil der Impedanz sind aber zum Teil gegensätzlich.

Die Messungen über einen definierten Zeitraum machen zudem deutlich, dass bei Invertierung der Daten die in beiden Versuchen auftauchende Einlaufphase ausgespart werden sollte. Vergleicht man die sweeps aus diesem Zeitraum, dem Zeitraum außerhalb der Einlaufphase, aber ohne Wurzeln, und dem Zeitraum, in dem Wurzeln vorhanden sind, so lässt sich der hochohmige Start mit raschem Absinken des Realteils nicht mit dem Wurzelwachstum erklären (siehe Abbildung 4.27). Unterstellt man, dass dieser Effekt nicht mit dem Wurzelwachstum, sondern mit einem anderen pflanzenphysiologischen Effekt zusammenhängt, darf sich die Auswertung der impedanzspektroskopischen Messungen (Invertierung) nicht auf diesen Bereich beziehen, um Wurzelwachstum zu beobachten. Diese Erkenntnis ist elementar, da in den meisten Veröffentlichungen unmittelbar mit den ersten Messungen begonnen wird.

Weiterhin sind die Messungen zeitlich nicht hochauflösend genug (zweimal wöchentlich über einen Monat (Repo u.a. (2005)). Die Aussage der Anfangsspektren ist damit anzuzweifeln.

Da im Hauptversuch 2 (Abschnitt 3.3.5 nahezu dieselben Randbedingungen herrschen, wie in der Arbeit von Cao u.a. (2011), sollen die Ergebnisse dieser beiden Untersuchungen hier direkt miteinander verglichen werden.

Abbildung 5.2 zeigt einerseits Messungen aus dem Hauptversuch 2, die bereits in Abschnitt 4.3 .3 beschrieben wurden (Messdaten), andererseits Daten, die mit Hilfe des bereits erwähnten Programmes Impedanz erzeugt wurden (Modelldaten ohne Wurzeln und Modelldaten mit Wurzeln). Die Daten aus der Veröffentlichung von Cao u.a. (2011) bilden dabei die Grundlage für die Generierung der Impedanzspektren. Die Impedanzspektren entsprechen also idealen Messreihen. Abgebildet sind hier Modelldaten, zum einen für einen Steckling ohne Wurzeln (Modell aus Abbildung 2.8a), zum anderen für einen Steckling mit Wurzeln (Modell aus Abbildung 2.8b).

Die Messdaten in dieser Abbildung sind beispielhafte Impedanzspektren von Steckling 3 vom 21.07.2012 bis 14.08.2012. Die durchgezogenen Linien entsprechen dem Messbereich von $60 \mathrm{~Hz}-$ $6 \times 10^{4} \mathrm{~Hz}$, der für die impedanzspektroskopischen Messungen bei Cao u.a. (2011) gewählt wurde, die gestrichelten Linien entsprechen dem Frequenzbereich von $1 \mathrm{~Hz}-10^{6} \mathrm{~Hz}$, in dem in dieser Arbeit gemessen wurde. Die gemessenen Daten wurden darüber hinaus durch die bereits erläuterte Differenzbildung verändert, um so die Systemfunktion herauszurechnen.

Vergleicht man zunächst nur die Messdaten jeden einzelnen Tages, so erkennt man in der komplexen Ebene, wie bereits bei der frequenzweisen Auswertung der Messdaten in Abbildung 4.29 gesehen, dass zu Beginn des Versuchs die Spektren hochohmig sind (21.07.2012) und rasch niederohmiger werden. Nach dem Austreiben der Wurzeln (ab 25.07.2012), in der Phase des stärksten Wurzelwachstums, sind geringere Unterschiede der Spektren zu erkennen. 


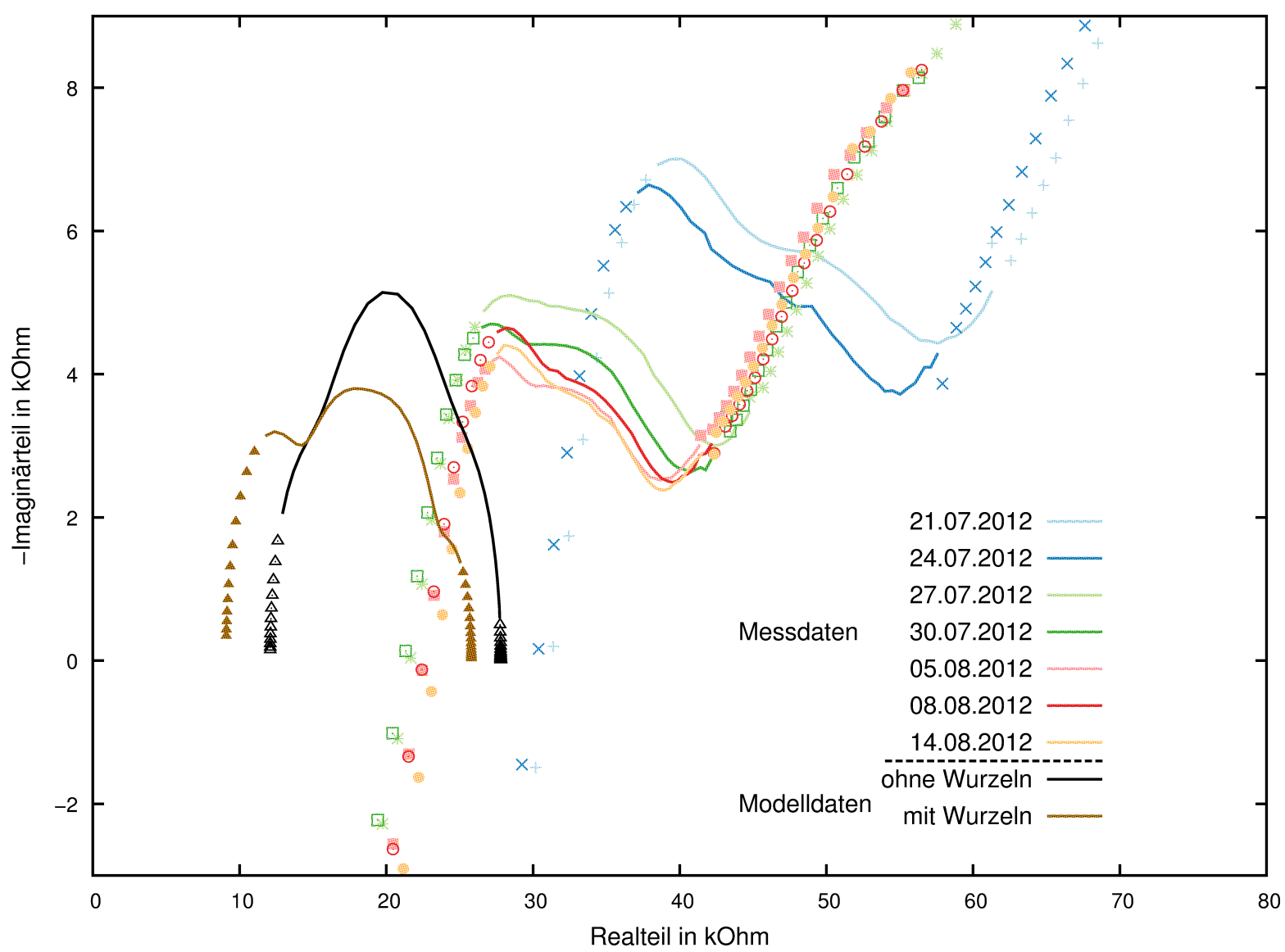

Abbildung 5.2: Vergleich von Modelldaten aus Cao u. a. (2011) mit Messdaten aus Hauptversuch 2. Genauere Erläuterungen zu dieser Abbildung finden sich im Text

Verglichen werden können weiterhin die Daten vom 21.07.2012-27.07.2012 mit den Modelldaten ohne Wurzeln. An den beiden genannten Tagen waren noch keine Wurzeln vorhanden. Die restlichen Daten vom 27.07.2012-14.08.2012 sind vergleichbar mit den Modelldaten mit Wurzeln. Mit dem Auftreten der ersten Wurzeln am 25.07.2012 fand das Hauptwurzelwachstum dieses Stecklings im oben genannten Zeitraum statt.

Der Vergleich macht deutlich, dass sich die Modelldaten von denen in dieser Arbeit entstandenen reellen Messdaten quantitativ wie qualtitativ unterscheiden. Die reellen Messdaten sind insgesamt deutlich hochohmiger hinsichtlich Real- und Imaginärteil.

Betrachtet man nur den Bereich von $60 \mathrm{~Hz}-6 \times 10^{4} \mathrm{~Hz}$ (durchgezogene Linien) der Messdaten und der Modelldaten des bewurzelten Stecklings, so wird deutlich, dass in weiten Teilen ein anderer Verlauf zu erkennen ist. Betrachtet man den höherfrequenten Bereich, so ist ein ähnlicher Trend zu erkennen (linke Bildhälfte). Der niederfrequente Bereich zeichnet sich durch einen deutlich gegensätzlichen Verlauf der Graphen auf. Der Imaginärteil der Impedanz steigt im hochfrequenten, wie auch im niederfrequenten Bereich deutlich an (bis ca. $16 \mathrm{k} \Omega$ ). Die Vorwärtsdaten zeigen ein gegensätzliches Bild. Hier sinkt der Imaginärteil zu beiden Seiten des Frequenzbereiches. Der Anstieg der Impedanz im niederfrequenten Bereich ist mit dem bereits erwähnten Polarisationseffekt erklärbar. Die Modelldaten von Cao u.a. (2011) zeigen keinen Polarisationseffekt, obwohl mit gleicher Messkonfiguration und vergleichbaren Elektroden ge- 
messen wurde.

An dieser Stelle soll jedoch erwähnt werden, dass die beobachteten Polarisationseffekte im niederfrequenten Bereich erkennbar sind $(<100 \mathrm{~Hz})$. Da in der Veröffentlichung von Cao u. a. (2011) im Frequenzbereich $60 \mathrm{~Hz}-6 \times 10^{4} \mathrm{~Hz}$ gemessen wird, erscheint der Polarisationseffekt folglich kleiner als bei Messungen im Frequenzbereich dieser Arbeit. Möglicherweise wurde der Frequenzbereich, der noch einen geringen Polarisationseffekt aufweist $(60 \mathrm{~Hz}-100 \mathrm{~Hz})$, bei der Modellierung der Impedanzspektren bei Cao u. a. (2011) ausgespart und als Messfehler nicht weiter beachtet.

Das von Cao u. a. (2011) vorgeschlagene Modell für einen bewurzelten Steckling kann bei den in dieser Arbeit durchgeführten Messungen im Frequenzbereich von $60 \mathrm{~Hz}-6 \times 10^{4} \mathrm{~Hz}$ angewandt werden. Die Messdaten und die Vorwärtsdaten für den unbewurzelten Steckling unterscheiden sich jedoch deutlicher. Hier ist der Verlauf der Kurven nur im Bereich des Hochpunktes ähnlich. An der Peripherie (links und rechts) der durchgezogenen Messlinie ist ein gegensätzlicher Verlauf zu verzeichnen. Obwohl also sämtliche Randbedingungen für den Hauptversuch 2 denen von Cao u. a. (2011) angeglichen wurden, unterscheiden sich die Ergebnisse von denen aus dieser Arbeit deutlich. Das hier vorgestellte Verfahren ist weniger invasiv, berücksichtigt wichtige Einflussgrößen und ist mit deutlich mehr Daten (z. T. mit minütlichen sweeps) hinterlegt. Trotz allem konnten die Ergebnisse von Cao nicht reproduziert werden. Die Ergebnisse zum Einfluss des Wasserstandes aus Abschnitt 4.3 .2 machen zudem deutlich, dass elektrischer Strom zumindest bei der Beprobung von unbewurzelten Weidenstecklingen offenbar über die Stecklingsrinde geleitet werden kann.

Ob der elektrische Strom bei bewurzelten Stecklingen unter diesen Rahmenbedingungen allerdings ebenso über die Mantelfläche des Stecklings geleitet wird oder tatsächlich über die Wurzeln mit der Wurzelhaarzone, gemäß dem Prinzip des geringsten Widerstandes, bleibt an dieser Stelle offen. Untersuchungen zu Wasserstandsänderungen an bewurzelten Stecklingen wurden aufgrund messtechnischer Schwierigkeiten nicht durchgeführt. Möglicherweise sind die verschiedenen Transportwege auch abhängig von der betrachteten Frequenz.

\section{Invertierung der Daten}

Die in dieser Arbeit untersuchten Modelle beeinhalten allesamt keine Induktivitäten, sondern lediglich ohmsche Widerstände und Kapazitäten.

Das Vorherrschen von Kondensatoren ergibt sich aus der Tatsache, dass bei den impedanzspektroskopischen Untersuchungen dieser Arbeit überwiegend negative Phasenwinkel gemessen wurden (siehe dazu auch Abschnitt 2.5). Lediglich im Bereich sehr hoher bzw. sehr niedriger Frequenzen wurden positive Phasenwinkel ermittelt. Diese positiven Phasenwinkel, die das Vorkommen von Induktivitäten vermuten lassen, wurden bei der Modellierung nicht beachtet. Positive Phasenwinkel wurden als Messfehler gewertet (siehe Abschnitt 5.3).

Beschäftigt man sich mit der Modellierung impedanzspektroskopischer Daten von Pflanzen, kann ein Blick in die Literatur zur Impedanzspektroskopie an tierischen Zellen hilfreich sein. Tierische Zellen und Zellverbände sind mittels der Impedanzspektroskopie wesentlich umfassender untersucht worden als pflanzliche. Zwar gibt es eine Reihe bedeutender Unterschiede im 


\section{Diskussion}

Aufbau von Pflanzen- und Tierzellen, jedoch existieren auch Gemeinsamkeiten wie die Existenz von Biomembranen. Modellhaft können tierische Biomembranen durch parallele Kapazitäten und ohmsche Widerstände beschrieben werden (vergleiche Martinsen und Grimnes (2011)).

Bei der Betrachtung des Impedanzspektrums von tierischen Zellen werden je nach Frequenz verschiedene Bereiche differenziert ( $\alpha, \beta$ und $\gamma$ Dispersionen), die verschiedenen Objektparametern zugesprochen werden können (Schwan (1994)). Für pflanzliche Zellverbände ist diese Zuordnung bisher aber noch nicht beschrieben worden.

Berücksichtigt man das Vorgehen bei der Modellanpassung für die hier vorgenommenen Untersuchungen an Pflanzenzellen, gemäß Abbildung 2.6, so gibt es eine Vielzahl möglicher Modelle, die die Messdaten ähnlich gut erklären. Die Erklärung der Messdaten durch verschiedene Modelle kommt in dieser Arbeit besonders in Abbildung 4.34 in den unteren beiden Teilgrafiken zum Ausdruck. Dieselben Daten können hier in ähnlicher Qualität durch zwei verschiedene Ersatzschaltbilder (Modelle) erklärt werden. Das von Ozier-Lafontaine und Bajazet (2005) gefundene Ersatzschaltbild (Abbildung 2.9) und das Ersatzschaltbild von Cao u.a. (2011) (Abbildung 2.8b liefern ähnliche gute Anpassungsergebnisse bei denselben Daten des zweiten Hauptversuchs.

Bei der einfachen Betrachtung der Anpassungsergebnisse erscheinen also beide Modelle passend. Beachtet man jedoch den Transportweg des elektrischen Stroms zwischen Steckling zur Nährlösung, so erscheint das Modell aus der Veröffentlichung von Cao u.a. (2011) (Abbildung 2.8b) deutlich passender als das von Ozier-Lafontaine und Bajazet (2005). Da der Strom auf dem genannten Transportweg durch Zellen hindurchfließt, helfen hier auch die Erkenntnisse der Untersuchungen von Zhang und Willison (1991). In dieser Veröffentlichung werden physikalische Modelle von Pflanzenzellen in Form von Ersatzschaltbildern untersucht. Als das passenste, sowohl aus physikalischer, als auch aus biologischer Sicht, stellte sich das bereits genannte double shell model (Abbildung 2.7) heraus. Cao u. a. (2011) greifen wesentliche Anordnungen der Impedanzelemente in dem von ihnen vorgeschlagenen Ersatzschaltbild auf.

Im Unterschied zur Veröffentlichung von Cao u. a. (2011) zeigt sich jedoch in der vorliegenden Arbeit, dass das Modell für bewurzelte Stecklinge (vgl. Abbildung 2.8b) über den gesamten Versuchsverlauf des zweiten Hauptversuchs das passendste ist. Die Impedanzspektren unbewurzelter und bewurzelter Stecklinge unterscheiden sich folglich kaum, wodurch gerechtfertigt ist, für beide Zustände das gleiche Modell zu wählen.

Weitere Modelle wurden nicht untersucht. Die Wahrscheinlichkeit mit anderen Ersatzschaltbildern ähnlich gute Anpassungen zu erreichen, ist aber groß.

Bei der multivariaten Analyse der Ergebnisse beider Hauptversuche fällt zunächst auf, dass sich die Ergebnisse der Invertierung der Stecklingsdaten eines jeden Versuchs z. T. höchstsignifikant unterscheiden, obwohl die Versuchsbedingungen für die Stecklinge jeweils gleich sein sollten. Dieser Befund lässt sich wie bereits erwähnt mit geringfügig unterschiedlichen Durchmessern der verschiedenen Stecklinge erklären. Wie in Abschnitt 4.3 .2 gezeigt werden konnte, korellieren Durchmesser und Real- bzw. Imaginärteil miteinander. Eine weitere mögliche Erklärung könnte der unterschiedliche Beginn der Wurzelbildung bzw. die Gesamtlänge der Wurzeln sein. So fällt z. B. Steckling 15 aus Hauptversuch 1 auf, der erst am Ende des Ver- 
suchszeitraums Wurzeln ausbildete und dementsprechend wenig Datenmaterial zur Verfügung stand.

Bei der Betrachtung der verschiedenen Modellparameter bei der multivariaten Analyse für Hauptversuch 1 und 2 fällt auf, dass einige dieser Parameter gut mit der Wurzellänge erklärbar sind. Hier ist jedoch deutlich zu sagen, dass das dieser Analyse zugrundeliegende statistische Modell maximale Freiheiten in der Wahl seiner Funktion hatte, so dass die Ausgleichskurve unter biologischen und physikalischen Gesichtspunkten zum Teil nicht zu erklären ist.

Speziell für die Untersuchung der Befunde der multivariaten Analyse für Hauptversuch 1 (Abbildung 4.33 fällt auf, dass nur wenige Modellparameter mit der Wurzellänge korrelieren. Die Modellparameter R0 und C1 fallen dabei besonders durch ihre hohen Signifikanzen auf. Der ohmsche Widerstand steigt mit Zunahme der Wurzellänge tendenziell linear. Dieser Befund ist zunächst verwunderlich, da mit zunehmender Wurzellänge der ohmsche Anteil der Impedanz aufgrund der größeren Kontaktfläche sinken müsste. Möglicherweise schirmen die äußeren Zellen der Feinwurzeln den elektrischen Stromfluss nach außen jedoch besser ab, als bisher vermutet, so dass mit zunehmender Länge der ohmsche Widerstand aufgrund der vergleichsweise hochohmigen Leitgewebe steigt (vgl. Abbildung 4.21).

Für Hauptversuch 2 (Abbildung 4.35 und Abbildung 4.36 fällt auf, dass die Zusammenhänge zwischen Wurzellänge und den jeweiligen Modellparametern unterschiedlich sind, obwohl mit dem gleichen Modell bei der Invertierung der Messergebnisse gearbeitet wurde. Hier ist darauf zu verweisen, dass in zwei verschiedenen Messvarianten (Zweipunktmessung und Dreipunktmessung) gemessen wurde und daher, wie in Abbildung 4.31 gesehen, deutlich unterschiedliche Impedanzspektren erzeugt wurden. Dieses Ergebnis bedeutet weiterhin, dass nur solche Ergebnisse miteinander verglichen werden können, bei denen sämtliche Randbedingungen gleich sind, insbesondere die Elektrodenanordnung.

Wie für den Hauptversuch 1 gesehen, gilt auch bei Hauptversuch 2, dass einige ohmsche Widerstände dieser Modelle positiv mit dem Längenwachstum der Wurzeln korrelieren (in beiden Messvarianten R5 und R3). Eine mögliche Erklärung ist bereits oben erläutert. Ein gegensätzliches Bild liefert bspw. R1 und R2 bei der Betrachtung der Invertierungsergebnisse der Dreipunktmessung. Der sinkende ohmsche Widerstand bei Zunahme der Wurzellänge folgt hier den physikalischen Gesetzmäßigkeiten vom Zusammenhang zwischen der Kontaktfläche eines Leiters und dem ohmschen Widerstand. Bei der Betrachtung der Kapazitäten können nur wenige eindeutige Zusammenhänge gefunden werden.

Der grundsätzliche Trend, dass bei zunehmender Wurzellänge die Kapazitäten sinken (z. B. C5 bei der Auswertung der Dreipunktmodelldaten, bzw. C1 bei den Zweipunktmodelldaten) unterscheidet sich von den Ergebnissen von Cao u. a. (2011), in denen ein gegensätzlicher Trend gefunden wurde.

Eine Zunahme der Wurzellänge geht, wie bei der fotografischen Auswertung gesehen, auch mit der Ausbildung Wurzeln zweiter Ordnung einher. Somit erhöht sich die Anzahl von Biomembranen, was wiederum einen Anstieg der Kapazität im Gesamtsystem zur Folge hätte. Dies ist allerdings mit den hier gemessenen Daten nicht zu belegen.

Bei beiden Hauptversuchen wird weiterhin deutlich, dass eher die ohmschen Widerstände 


\section{Diskussion}

mit der Wurzellänge korrelieren als die Kapazitäten. Bei Cao u. a. (2011) korrelierten die Kapazitäten (besonders C3) mit der Wurzeloberfläche. An dieser Stelle sei darauf verwiesen, dass in dieser Arbeit die Wurzellänge, bei Cao u. a. (2011) die Wurzeloberfläche mit Modellparametern korreliert wird. Aufgrund des Zusammenhanges zwischen Wurzellänge und Wurzeloberfläche (vgl. Abbildung 4.24), können die Ergebnisse jedoch verglichen werden.

Bei der multivariaten Analyse der Zweipunktmodelldaten des Hauptversuches 2 sind, wie bereits erwähnt, einige deutliche Zusammenhänge zwischen Wurzellänge und Modellparametern gefunden worden. Dieser Befund verwundert, da die bereits erwähnten Störgrößen (Übergangswiderstände und Polarisationseffekte) im besonders hohen Maße bei der Zweipunktmessung auftreten und zunächst vermutet wurde, dass diese Effekte die eigentlich interessierenden Größen überlagern könnten. Offenbar ist es aber aufgrund dieses nahezu störungsfreien Messverfahrens und der Differenzbildung der Spektren mit anschließender Invertierung auch hier möglich, Zusammenhänge nachzuweisen.

Geht man davon aus, dass elektrischer Strom von der Pflanze über den gleichen Weg aufgenommen und in der Pflanze weitertransportiert wird wie Wasser (bzw. Nährlösung), passiert der elektrische Strom zwangsläufig Zellen mit den entsprechenden Zellwänden und -membranen. Dvořák u.a. (1981) gehen bereits Anfang der 1980er Jahre davon aus, dass elektrischer Strom bei wechselnder Frequenz über unterschiedliche Wege (apoplastisch und symplastisch) transportiert wird. Misst man wie in der vorliegenden Arbeit über einen weiten Frequenzbereich, müssen bei der Modellbildung auch beide Wege mit den entsprechenden Impedanzelementen auftauchen.

Neben Biomembranen, die sich als Kapazitäten modellieren lassen, werden weitere Zellbestandteile und -organellen durchwandert wie Zellwände, extrazellulärer Raum, Plasmodesmen etc. Diese lassen sich physikalisch vereinfacht als ohmsche Widerstände abbilden (Zhang und Willison (1991)). In dieser Arbeit wurde das double shell model nicht durch eigene Messungen auf Zellebene überprüft.

In anderen Arbeiten mit impedanzspektroskopischen Untersuchungen konnte gezeigt werden, dass häufig ein einzelner ohmscher Widerstand als Summe von Einzelwiderständen (Leitungen, Elektroden etc.) verstanden werden kann (z. B. Hoffmann-Walbeck (2007)). Ein solcher solitär stehender ohmscher Widerstand findet sich auch z. B. bei Ozier-Lafontaine und Bajazet (2005) und wird mit dem elektrischen Widerstand des Substrates erklärt.

Insgesamt lässt sich festhalten, dass die Invertierung der Daten erst dann erfolgen sollte, wenn auf eine möglichst belastbare Datengrundlage, sowohl quantitativ als auch qualitativ zurückgegriffen werden kann.

Am belastbarsten stellen sich dabei die Daten aus der Dreipunktmessung des zweiten Hauptversuches dar, da hier sämtliche Randbedingungen berücksichtigt wurden und auf eine hohe Datenmenge zurückgegriffen werden kann.

Die statistische Auswertung der Modelldaten brachte hier zum Teil hochsignifikante Zusammenhänge zwischen Modellparametern und der Wurzellänge. Das Modell von Cao u.a. (2011) (Abbildung 2.8b konnte dabei gut auf die entstandenen Daten übertragen werden. Bei Korellation der Modelldaten und der Wurzellänge fallen jedoch besonders die ohmschen 
Widerstände mit hohen Signifikanzen auf und weniger die Kapazitäten. Die ohmschen Widerstände zeigen allerdings teilweise gegensätzliche Zusammenhänge. Diese Befunde erstaunen, da sie im Gegensatz zu zahlreichen Veröffentlichungen stehen, bei denen Kapazitäten mit der Wurzeloberfläche korrelieren.

An dieser Stelle bleibt offen, warum sich die Divergenzen zwischen der vorliegenden Arbeit und der veröffentlichten Studie (Cao u.a. (2011)) ergeben. Da in der vorliegenden Arbeit qualitativ hochwertige Daten in sehr hoher Menge generiert wurden, sollten die Ergebnisse der genannten Veröffentlichung kritisch betrachtet werden. 



\section{Fazit und Ausblick}

Die Eignung monofrequenter Messverfahren (Erdungsmessung und Bodenwiderstandsmethode) zur Feinwurzelquantifizierung erscheint aufgrund der hier durchgeführten Tests widerlegt. Zwar existieren Zusammenhänge zwischen Baumdurchmesser und Erdungswiderstand, die oberflächlich betrachtet mit der höheren Feinwurzeloberfläche durchmesserstärkerer Bäume erklärt werden können, bei näherem Hinsehen jedoch aufgrund der größeren Durchmesser per se begründet werden konnten. Der Maskierungseffekt des Stammwiderstandes zeigt die Grenzen dieser Methode zur Quantifizierung von Feinwurzeln auf, woraus sich die Fokussierung dieser Arbeit auf die Impedanzspektroskopie ableitet, bei der die verschiedenen Einflussgrößen getrennt voneinander betrachtet werden können.

Die Erfassung und Beschreibung von Zusammmenhängen zwischen elektrischen Eigenschaften und biologischen Messwerten von Stecklingen mittels Impedanzspektroskopie ist im Hinblick auf deren Durchmesser und dem Realteil bzw. dem Imaginärteil gelungen. Direkte höchstsignifikante Zusammenhänge von der Wurzellänge und physikalischen Messwerten konnten bei den Roh- und Modelldaten gefunden werden. Die Zusammenhänge zwischen Wurzellänge und Realteil resp. Imaginärteil der Impedanz weisen bei unterschiedlichen Frequenzen in teils gegensätzliche Richtungen. Der positive Zusammenhang zwischen ohmschen Widerstand und Wurzellänge bei der modellhaften Betrachtung kann mit der Zunahme hochohmigen Leitgewebes erklärt werden.

Diese Ergebnisse können jedoch einschlägige Veröffentlichungen zu den hier betrachteten Methoden der letzten Jahre (Erdungsmessung, Bodenwiderstandsmethode und Impedanzspektroskopie) inhaltlich nicht stützen. Die vertiefende Erörterung dieses Befundes erscheint aufgrund der fehlenden Standardisierung der Messmethoden und Verfahren nicht sinnvoll. Problematisch erscheint die Tatsache, dass trotz identischen Versuchsdesigns die Ergebnisse der Veröffentlichung von Cao u.a. (2011) nicht reproduziert werden konnten.

Um belastbare Daten und Aussagen zu generieren, erscheint es unumgänglich, zunächst eine methodische Standardisierung unter Laborbedingungen zu erarbeiten. Dabei sollte es Ziel sein, Messungen auf dem Gebiet der Bodenwiderstandsmethode und der Impedanzspektroskopie so zu gestalten, dass die in dieser Arbeit gefundenen beeinflussenden Faktoren nicht nur qualitativ, sondern auch quantitativ Berücksichtigung finden. Erst wenn diese Parameter berücksichtigt worden sind, können weitere Messungen erfolgen. Diese Arbeit leistet somit einen ersten Beitrag dazu.

Erste Empfehlungen können für künftige impedanzspektroskopische Messungen formuliert werden:

- Wahl nicht polarisierbarer, inerter Elektroden bspw. Edelstahl- oder Silberelektroden

- Messung in Vierpunktmesstechnik 
6 Fazit und Ausblick

- möglichst substratnahe Platzierung der sprossseitigen Messelektrode

- Einstellung einer Nährlösung mit genau definierter, möglichst hoher Leitfähigkeit

- konstanter Wasserstand

- konstante Temperatur

- Wahl durchmesserstärkerer Stecklinge

Freilandmessungen erscheinen aufgrund der methodischen Unsicherheiten derzeit nicht sinnvoll. 


\section{Zusammenfassung}

Das Wurzelsystem einer Pflanze erfüllt lebenswichtige Funktionen wie bspw. die Verankerung des Baumes und die Aufnahme von Wasser und Nährstoffen. Die Fein- und Feinstwurzeln spielen eine besondere Rolle, da nur über sie die Aufnahme von Wasser möglich ist.

Die Quantifizierung insbesondere von Feinwurzeln bereitet jedoch große Schwierigkeiten, da diese im Gegensatz zu den oberirdischen Organen des Baumes unsichtbar sind.

In den letzten Jahren wurden daher verschiedene elektrische Verfahren entwickelt, Feinwurzeln mengenmäßig zu erfassen bzw. die durchwurzelte Fläche einer Pflanze abzuschätzen, ohne die Wurzeln auszugraben und zu beschädigen. Bei allen Verfahren wird zwischen der Pflanze und dem Substrat, in dem die Pflanze wächst, ein elektrischer Stromkreis angelegt. Mit Hilfe der ermittelten physikalischen Messwerten sollen Rückschlüsse auf die Dimension des Wurzelsystems gezogen werden. Diese Verfahren wurden in der vorliegenden Arbeit auf Bäume bzw. auf Stecklinge von Bäumen angewandt und kritisch auf ihre Tauglichkeit überprüft.

Die Erdungsmessung ist ein niederfrequentes elektrisches Verfahren. Die Methode wurde an verschiedenen Baumarten in Feldversuchen getestet. Diese Versuche hatten zum Ziel, die optimale Versuchsanordnung zu finden und mögliche Einflussfaktoren auf den Erdungswiderstand aufzudecken. Die Bodenwiderstandsmethode, eine Weiterentwicklung der Erdungsmessung und ebenfalls ein niederfrequentes Messverfahren, wurde in Laborexperimenten im elektrolytischen Trog mit Hilfe von Wurzelimitaten überprüft und zusätzlich in Feldversuchen getestet. Die Impedanzspektroskopie, ein multifrequentes Messverfahren, bildet den Schwerpunkt dieser Arbeit. Bei der Impedanzspektroskopie wurden einige Vorversuche durchgeführt, um das optimale Versuchsdesign zu ergründen, worauf zwei Hauptversuche folgten. Alle impedanzspektroskopischen Messungen wurden unter Laborbedingungen an Weidenstecklingen durchgeführt.

Die hier beschriebenen Versuche zur Erdungsmessung machen deutlich, dass aufgrund der notwendigen Eliminierung von Übergangswiderständen grundsätzlich in Vierpunktmesstechnik gemessen werden muss. Es stellte sich heraus, dass zahlreiche Faktoren, wie die Temperatur und die Bodenfeuchte, zusätzlich die durch Vierpunktmesstechnik ermittelten Werte beeinflussen. Als Haupteinflussfaktor wurde der elektrische Widerstand der Leitgewebe identifiziert. Der eigentliche Erdungswiderstand eines Baumes kann mit dieser Methode nicht separat von Störgrößen erfasst werden, was die Grenze der Methode für die Feinwurzelquantifizierung aufzeigt.

Bei der Bodenwiderstandsmethode hat sich gezeigt, dass Wurzelimitate (Kupferkabel resp. Kupferscheiben) im elektrolytischen Trog nur dann identifiziert werden können, wenn sie möglichst nah an der Elektrolytoberfläche platziert werden. Je tiefer das Wurzelimitat von dieser Oberfläche entfernt ist, desto ungenauer zeigt es sich im elektrischen Bild. Bei der Variation der Oberfläche der Kupferscheiben konnte beobachtet werden, dass größere Scheiben in größerer Tiefe ähnliche Ergebnisse wie kleinere Scheiben in geringerer Tiefe liefern. Da weder die Oberfläche der Wurzeln, noch die Tiefe der Wurzeln vor der Messung bekannt ist, können die 


\section{Zusammenfassung}

Ergebnisse also nicht eindeutig interpretiert werden.

Bei der Impedanzspektroskopie wurden wesentliche, in der Literatur bislang nicht berücksichtigte Faktoren aufgedeckt, die in unterschiedlicher Intensität Einfluss auf das Impedanzspektrum nehmen. Für die Elektrodenanordnung, den Wasserstand, die Dimension der Messobjekte und das Leitgewebe der Stecklinge wurde ein deutlicher Einfluss auf die Messergebnisse gefunden, während die anderen Faktoren (Elektrodenwahl, Leitfähigkeit der Nährlösung, Temperatur) einen geringeren Einfluss auf die Messergebnisse haben.

Bei der Korrelation der Rohdaten (Imaginärteil und Realteil der Impedanz) mit der Wurzellänge wurden zum Teil hochsignifikante Zusammenhänge bei der Verwendung einer Quantilregression gefunden. Es wurden Modelle gefunden, die die gemessenen Impedanzspektren gut erklären. Alle Modelle setzen sich überwiegend aus sogenannten RC-Gliedern, Parallelschaltungen von Widerständen und Kondensatoren unterschiedlicher Anzahl zusammen. Die Widerstände und wenige Kapazitäten korellieren dabei mit der Wurzellänge. Diese Ergebnisse unterscheiden sich jedoch deutlich von den Ergebnissen einschlägiger Veröffentlichungen.

Insgesamt lässt sich festhalten, dass es sowohl mit der Erdungsmessung, als auch mit der Bodenwiderstandsmethode nicht möglich war, das Wurzelsystem zu quantifizieren. Bei der Impedanzspektroskopie bedarf es einer Standardisierung der Messmethode. Bei den impedanzspektroskopischen Messungen gibt es deutliche Zusammenhänge zwischen physikalischen Messwerten und der Wurzellänge, die jedoch in unterschiedliche Richtungen weisen und im Gegensatz zu bereits veröffentlichten Ergebnissen stehen. Daraus ergibt sich, dass weitergehende Untersuchungen, insbesondere zu den Randbedingungen von elektrischen Verfahren zur Quantifizierung von Feinwurzeln, besonders der Impedanzspektroskopie, folgen müssen. Diese Arbeit kann als ein Schritt hin zur methodischen Fundierung betrachtet werden. 


\section{Literatur}

Agarwal, B. K. u.a. (1979). „X-ray Spectroscopy“. In: Springer series in optical sciences 15, S. $35-46$.

Aubrecht, L., Z. Staněk und J. Koller (2006). „Electrical measurement of the absorption surfaces of tree roots by the earth impedance method: 1. Theory“. In: Tree Physiology 26, S. $1105-1112$.

Aulen, M. und B. Shipley (2012). „Non-destructive estimation of root mass using electrical capacitance on ten herbaceous species“. In: Plant and Soil, S. 1-9.

Balder, H. (1998). Die Wurzeln der Stadtbäume: Ein Handbuch zum vorbeugenden und nachsorgenden Wurzelschutz. Parey Buchverlag im Blackwell Wissenschafts-Verlag.

Barsoukov, E. und J.R. MacDonald (2005). Impedance spectroscopy: theory, experiment, and applications. Wiley-Interscience.

Beem, J. van, M.E. Smith und R.W. Zobel (1998). „Estimating root mass in maize using a portable capacitance meter". In: Agronomy Journal 90.4, S. 566-570.

Boukamp, B. A. (1989). Equivalent Circuit: Users Manual. University of Twente, Department of Chemical Technology.

Brasse, H. (2002). „Angewandte Geophysik für Nebenfächler (geoelektrische Verfahren)“. In: Manusskript zur Vorlesung, Freie Universität Berlin, Fachrichtung Geophysik.

Bresinsky, A. u. a. (2008). Strasburger, Lehrbuch der Botanik. 36. Springer Spektrum Akademischer Verlag.

Cao, Y. (2011). „Biomass production and control of nutrient leaching of willows using different planting methods with special emphasis on an appraisal of the electrical impedance for roots". Diss. School of Forest Sciences Faculty Science und Forestry University of eastern Finland.

Cao, Y. u. a. (2011). „Analysis of the willow root system by electrical impedance spectroscopy“. In: Journal of Experimental Botany 62.1, S. 351-358.

Čermák, J. u. a. (2006). „Electrical measurement of tree root absorbing surfaces by the earth impedance method: 2. Verification based on allometric relationships and root severing experiments“. In: Tree Physiology 26.9, S. 1113-1121. 


\section{Literatur}

Čermák, J. u.a. (2013). „Absorptive root areas of large pedunculate oak trees differing in health status along a road in South Bohemia, Czech Republic“. In: Urban Forestry and Urban Greening 12, S. 238-245.

Chloupek, O. (1972). "The relationship between electric capacitance and some other parameters of plant roots“. In: Biologia Plantarum 14.3, S. 227-230.

- (1977). „Evaluation of the size of a plant's root system using its electrical capacitance“. In: Plant and Soil 48.2, S. 525-532.

Dalton, F.N. (1995). „In-situ root extent measurements by electrical capacitance methods“. In: Plant and Soil 173.1, S. 157-165.

Davey, Ch.L. und D.B. Kell (1998a). „The influence of electrode polarisation on dielectric spectra, with special reference to capacitive biomass measurements: I. Quantifying the effects on electrode polarisation of factors likely to occur during fermentations". In: Bioelectrochemistry and bioenergetics 46.1, S. 91-103.

- (1998b). „The influence of electrode polarisation on dielectric spectra, with special reference to capacitive biomass measurements::(II) Reduction in the contribution of electrode polarisation to dielectric spectra using a two-frequency method“. In: Bioelectrochemistry and bioenergetics 46.1, S. 105-114.

Dean, D.A. u.a. (2008). „Electrical impedance spectroscopy study of biological tissues“. In: Journal of electrostatics 66.3 , S. 165-177.

Deckwart, J. M. (2007). „Impedanzspektroskopie bei schneller Korngrenzdiffusion am Beispiel des Silbertellurids“. Diss. Universität Münster.

Doerner, P. (1998). „Root development: quiescent center not so mute after all“. In: Current biology 8.2, R42-R44.

Du, Q. (1991). „Einfluss holzartspezifischer Eigenschaften auf die elektrische Leitfähigkeit wichtiger Handelshölzer“. Diss. Universität Hamburg.

Dvořák, M., J. Černohorská und K. Janáček (1981). „Characteristics of current passage through plant tissue“. In: Biologia Plantarum 23.4, S. 306-310.

Ellis, T., W. Murray und L. Kavalieris (2012). „Electrical capacitance of bean (Vicia faba) root systems was related to tissue density- a test for the Dalton Model“. In: Plant and Soil, S. $1-10$.

Ende, D. und K.M. Mangold (1993). „Impedanzspektroskopie“. In: Chemie in unserer Zeit 27.3, S. $134-140$.

Fukuma, H., K. Tanaka und I. Yamaura (2003). „An Evaluation Method of Ground Resistance of a Tree“. In: IEEJ Transactions on Electronics, Information and Systems 123, S. 629-630. 
Hanskötter, B. (2004). Diagnose fakultativer Farbkerne an stehender Rotbuche (Fagus sylvatica L.) mittels "Elektrischer Widerstandstomographie". Cuvillier Verlag.

Hayden, R.I. u.a. (1969). „Electrical impedance studies on potato and alfalfa tissue“. In: Journal of Experimental Botany 20.2, S. 177-200.

Hendrick, R. L. und K. S. Pregitzer (1992). "The demography of fine roots in a northern hardwood forest". In: Ecology, S. 1094-1104.

Hoffmann-Walbeck, W. (2007). „Anwendung der Impedanzspektroskopie für die Untersuchung von Biogasreformat in Brennstoffzellen“. Diss. Univ.-Bibliothek der Techn. Univ. Berlin.

Hofmann, M. (2009). „Integrierte Impedanzspektroskopie aerober Zellkulturen in biotechnologischen Hochdurchsatzscreenings“. Diss. Universitätsbibliothek RWTH Aachen.

Hruska, J., J. Čermák und S. Šustek (1999). „Mapping tree root systems with ground-penetrating radar“. In: Tree Physiology 19.2, S. 125-130.

Johnson, D. (2000). „Zplot, ZView Electrochemical Impedance Software, version 2.3 b, Scribner Associates". In: Inc., Southern Pines, NC, USA.

Joslin, J.D. und G.S. Henderson (1987). „Organic matter and nutrients associated with fine root turnover in a white oak stand“. In: Forest Science 33.2, S. 330-346.

Kasikci, I. (2013). Elektrotechnik für Architekten, Bauingenieure und Gebäudetechniker: Grundlagen und Anwendung in der Gebäudeplanung. Springer.

Kendall, W.A., G.A. Pederson und R.R. Hill Jr (1982). „Root size estimates of red clover and alfalfa based on electrical capacitance and root diameter measurements". In: Grass and Forage Science 37.3, S. 253-256.

Koenker, Roger (2015). quantreg: Quantile Regression. R package version 5.11. URL: http: //CRAN.R-project.org/package=quantreg.

Köstler, J.N., E. Brückner und H. Bibelriether (1968). Die Wurzeln der Waldbäume: Untersuchungen zur Morphologie der Waldbäume in Mitteleuropa. P. Parey.

Laasch, T. und E. Laasch (2013). Haustechnik: Grundlagen-Planung-Ausführung. Springer DE.

Lange, G. (2005). „Gleichstromgeoelektrik“. In: Methodenhandbuch Deponieuntergrund, S. 128174.

Lösch, R. (2001). Wasserhaushalt der Pflanzen. Quelle \& Meyer.

METRAWATT, GOSSEN (2007). Bedienungsanleitung; GEOHM 5. GOSSEN METRAWATT. GMC-I Messtechnik GmbH. Südwestpark 15, 90449 Nürnberg, Germany.

MacDonald, J.R. (1987). Impedance spectroscopy emphasizing solid materials and systems. John Wiley \& Sons New York. 


\section{Literatur}

MacDonald, J.R. (1999). „LEVM Manual ver. 7.11. CNLS Immittance Fitting Program“. In: Solartron Group Limited.

MacDougall, R.G., R.G. Thompson und H. Piene (1987). „Stem electrical capacitance and resistance measurements as related to total foliar biomass of balsam fir trees". In: Canadian Journal of Forest Research 17.9, S. 1071-1074.

Mancuso, S. (2011). Measuring roots: an updated approach. Springer.

Martinsen, O.G. und S. Grimnes (2011). Bioimpedance and bioelectricity basics. Access Online via Elsevier.

Matamala, R. u.a. (2003). „Impacts of fine root turnover on forest NPP and soil C sequestration potential“. In: Science 302.5649, S. 1385-1387.

Matsumoto, N. u. a. (2001). "Capacitance as a possible indicator for size of maize root system“. In: Proceedings of the 6th Symposium of the International Society of Root Research, S. 578579 .

McBride, R., M. Candido und J. Ferguson (2008). „Estimating root mass in maize genotypes using the electrical capacitance method". In: Archives of Agronomy and Soil Science 54.2, S. 215-226.

Müller, P. (2005). „Biomechanische Beschreibung der Baumwurzel und ihre Verankerung im Erdreich“. In: Wissenschaftliche Berichte FZKA 7104.

Multiphysics, COMSOL (2009). 3.5, COMSOL Inc.

Nabors, M. W. (2007). Botanik. Pearson Deutschland GmbH.

Nadezhdina, N. und J. Čermák (2003). „Instrumental methods for studies of structure and function of root systems of large trees“. In: Journal of Experimental Botany 54.387, S. 15111521.

Ozier-Lafontaine, H. und T. Bajazet (2005). „Analysis of root growth by impedance spectroscopy (EIS)“. In: Plant and Soil 277.1, S. 299-313.

Paszewski, A. u. a. (2006). „Resistance and capacity in the thallus of Marchantia polymorpha“. In: Physiologia Plantarum 54.2, S. 213-220.

Polomski, J. und N. Kuhn (2001). „Wurzelhabitus und Standfestigkeit der Waldbäume“. In: Forstwissenschaftliches Centralblatt vereinigt mit Tharandter forstliches Jahrbuch 120.1-6, S. 303-317.

Polomski, J. u.a. (2002). „Root research methods“. In: Plant roots: The hidden half, S. 295321. 
Preston, G.M. u.a. (2004). „Estimating root mass in young hybrid poplar trees using the electrical capacitance method“. In: Agroforestry systems 60.3, S. 305-309.

RCoreTeam (2012). R: A Language and Environment for Statistical Computing. R Foundation for Statistical Computing, Vienna, Austria, $200 \%$.

Raven, P.H. u. a. (2000). Biologie der Pflanzen. Walter de Gruyter.

Repo, T., J. Laukkanen und R. Silvennoinen (2005). „Measurement of the tree root growth using electrical impedance spectroscopy“. In: Silva Fennica 39.2, S. 159-166.

Richter, G. (1997). Stoffwechselphysiologie der Pflanzen: Physiologie und Biochemie des Primärund Sekundärstoffwechsels. 6. Aufl. Georg Thieme Verlag.

Schopfer, P. und A. Brennicke (2010). Pflanzenphysiologie. Spektrum Akademischer Verlag.

Schwan, H.P. (1963). „Electric characteristics of tissues“. In: Radiation and Environmental Biophysics 1.3, S. 198-208.

- (1994). „Electrical properties of tissues and cell suspensions: mechanisms and models“. In: Engineering in Medicine and Biology Society, 1994. Engineering Advances: New Opportunities for Biomedical Engineers. Proceedings of the 16th Annual International Conference of the IEEE. IEEE, A70-A71.

Stokes, A. u. a. (2002). „An evaluation of different methods to investigate root system architecture of urban trees in situ: I. ground-penetrating radar". In: Journal of Arboriculture 28.1, S. $2-10$.

Streckenbach, M. (2009). „Interaktionen zwischen Wurzeln und unterirdischer Infrastruktur Grundlagen und Strategien zur Problemvermeidung“. Diss. Ruhr-Universität Bochum.

Urban, J., R. Bequet und R. Mainiero (2011). „Assessing the applicability of the earth impedance method for in situ studies of tree root systems". In: Journal of Experimental Botany 62.6, S. $1857-1869$.

Vanninen, P. und A. Mäkelä (1999). „Fine root biomass of Scots pine stands differing in age and soil fertility in southern Finland“. In: Tree Physiology 19.12, S. 823-830.

Vianden, M.J. (2013). „Zerstörungsfreie Wurzelortung mit geophysikalischen Methoden im urbanen Raum“. Diss. Universität Göttingen.

Waisel, Y., A. Eshel, U. Kafkafi u. a. (2002). Plant roots: the hidden half. Ed. 3. Marcel Dekker Inc.

Waloschek, P. (1998). Wörterbuch Physik. dtv.

Wang, Jiahui u.a. (2014). robust: Robust Library. R package version 0.4-16. URL: http:// CRAN.R-project.org/package=robust 


\section{Literatur}

Weidelt, P. (2005). „Geoelektrik- Grundlagen Geoelektrik“. In: Methodenhandbuch Deponieuntergrund, S. 71-100.

Weiler, E.W. und L. Nover (2008). Allgemeine und molekulare Botanik. Thieme.

Williams, T., C. Kelley u. a. (2010). „Gnuplot 4.4: an interactive plotting program“. In: Official gnuplot documentation, http://sourceforge.net/projects/gnuplot.

WinRHIZO (2004). WinRHIZO Pro 2004a Software: Root Analysis.

Wood, S.N. (2011). „Fast stable restricted maximum likelihood and marginal likelihood estimation of semiparametric generalized linear models." In: Journal of the Royal Statistical Society (B) 73(1), S. 3-36.

Yamada, M. u. a. (2003). "Vector locus of an equivalent radius of the root in growing plants". In: Instrumentation and Measurement Technology Conference, 2003. IMTC'03. Proceedings of the 20th IEEE. Bd. 2. IEEE, S. 1294-1297.

Yamaura, I. u.a. (2000). „A measurement method of grounding impedance of a tree“. In: Instrumentation and Measurement Technology Conference, 2000. IMTC 2000. Proceedings of the 17th IEEE. Bd. 3. IEEE, S. 1163-1166.

Yamaura, I. u. a. (2002). „An estimation method of ground resistance of trees growing in different lands“. In: Instrumentation and Measurement Technology Conference, 2002. IMTC/2002. Proceedings of the 19th IEEE. Bd. 2. IEEE, S. 949-952.

Yamaura, I. u. a. (2006). „A Study on Ground Impedance of the Root in Plants". In: IEIC Technical Report (Institute of Electronics, Information and Communication Engineers) 106.253, S. 1-4.

Yanai, R.D., B.B. Park und S.P. Hamburg (2006). „The vertical and horizontal distribution of roots in northern hardwood stands of varying age". In: Canadian journal of forest research 36.2 , S. $450-459$.

Zhang, M. I. N. und J. H. M. Willison (1991). „Electrical Impedance Analysis in Plant Tissues“. In: Journal of Experimental Botany 42.11, S. 1465-1475.

Zimmermann, E. (2011). Phasengenaue Impedanzspektroskopie und-tomographie für geophysikalische Anwendungen. Universitäts- und Landesbibliothek Bonn. 


\section{A Anhang}
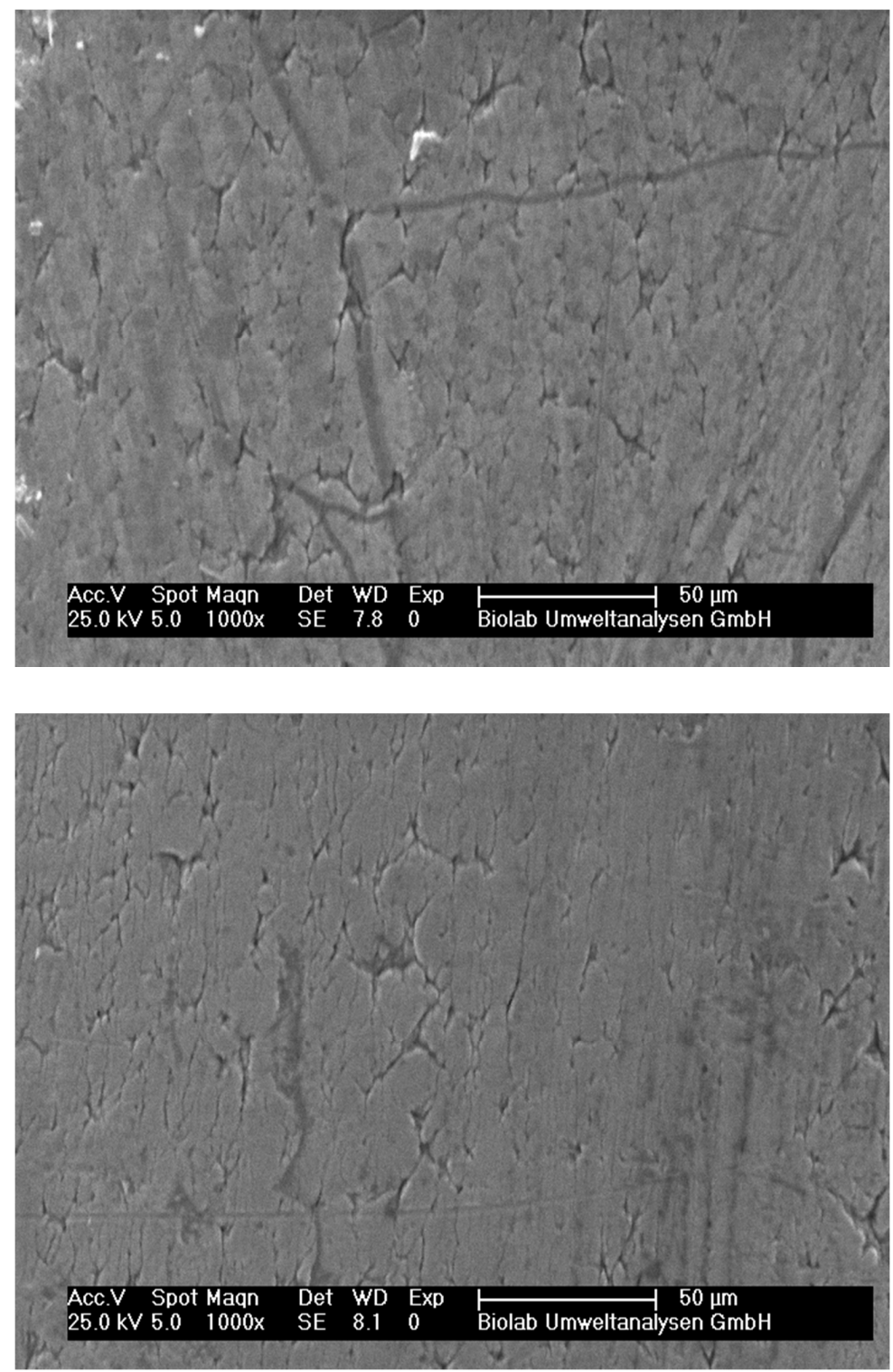

Abbildung A.1: Mit einem Rasterelektronenmikroskop aufgenommene Bilder der Edelstahlkanülenoberfläche; oben: Bereich, der im Steckling steckte, unten: Bereich außerhalb des Stecklings. 

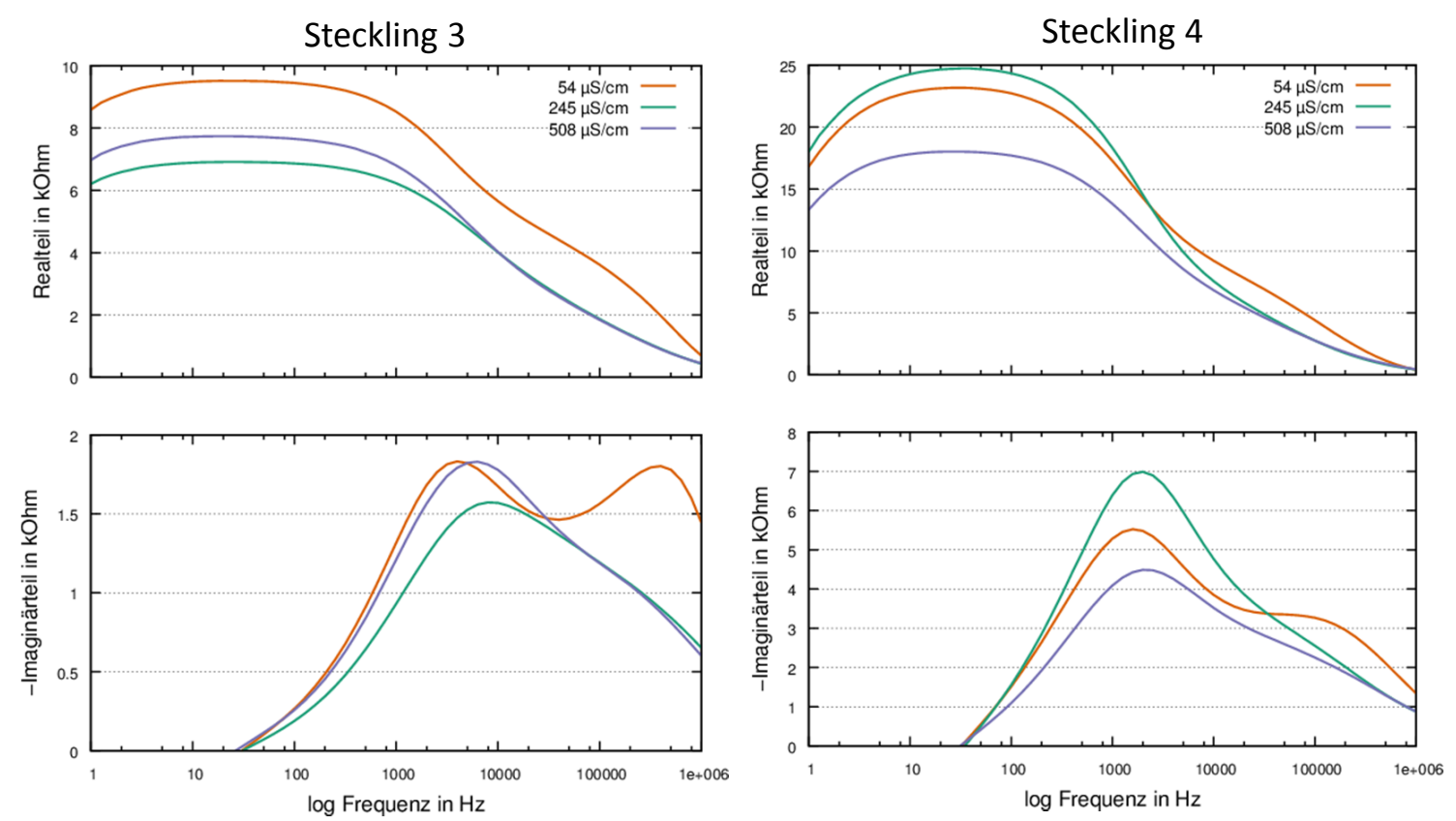

Steckling 5

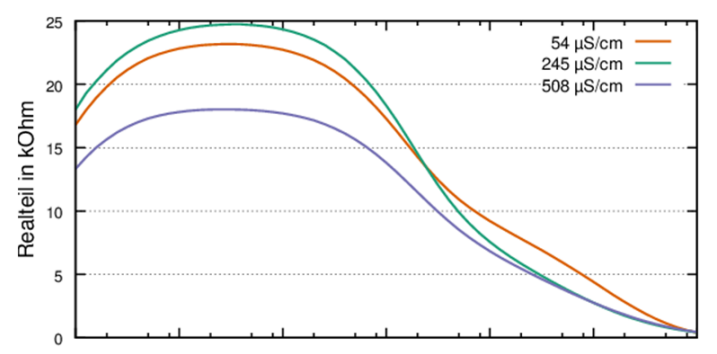

\section{Steckling 6}
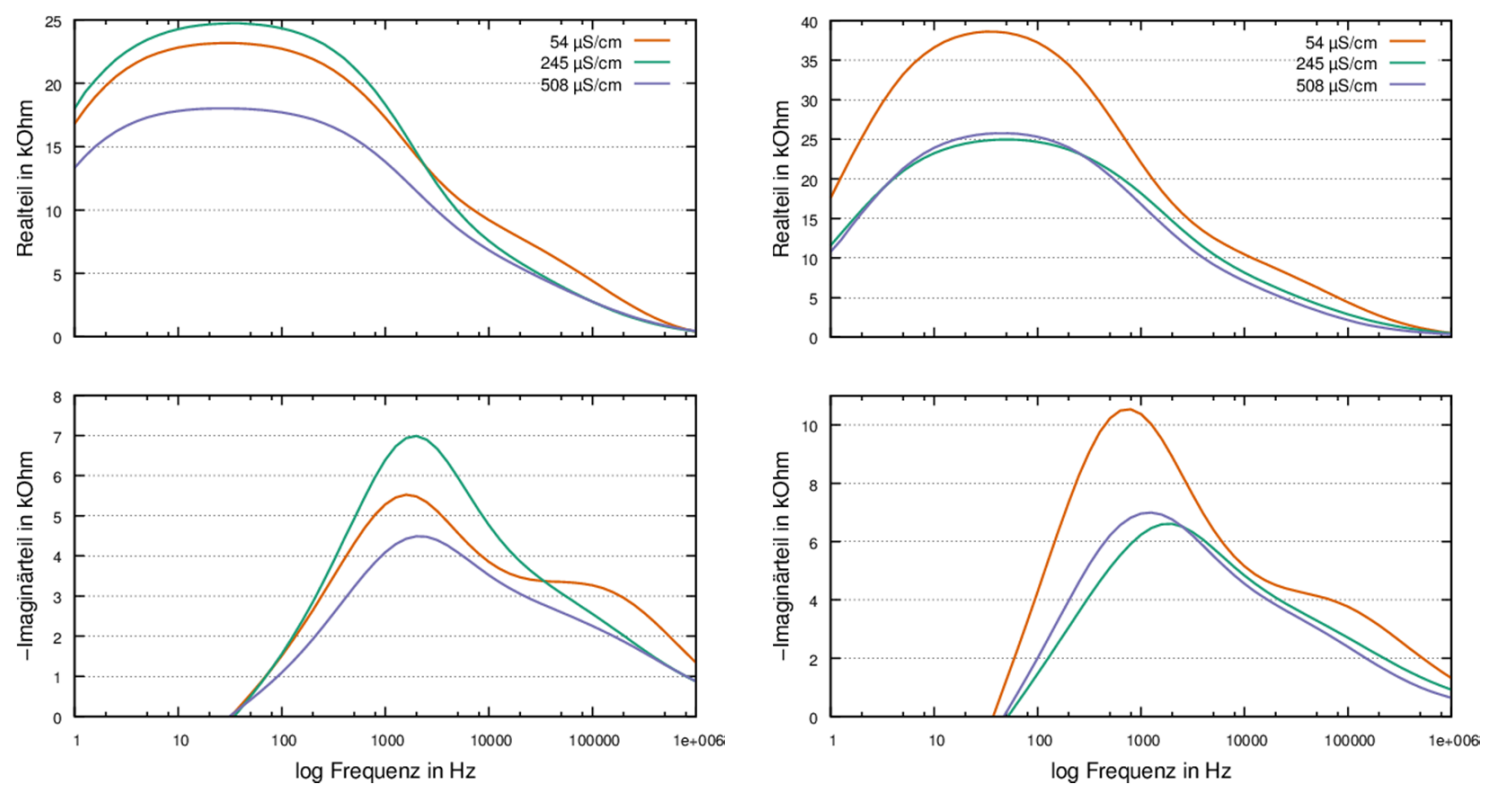

Abbildung A.2: Ergebnisse der Untersuchungen zum Einfluss der Leitfähigkeit der Nährlösung für die Stecklinge 3 bis 6 . 
Steckling 7
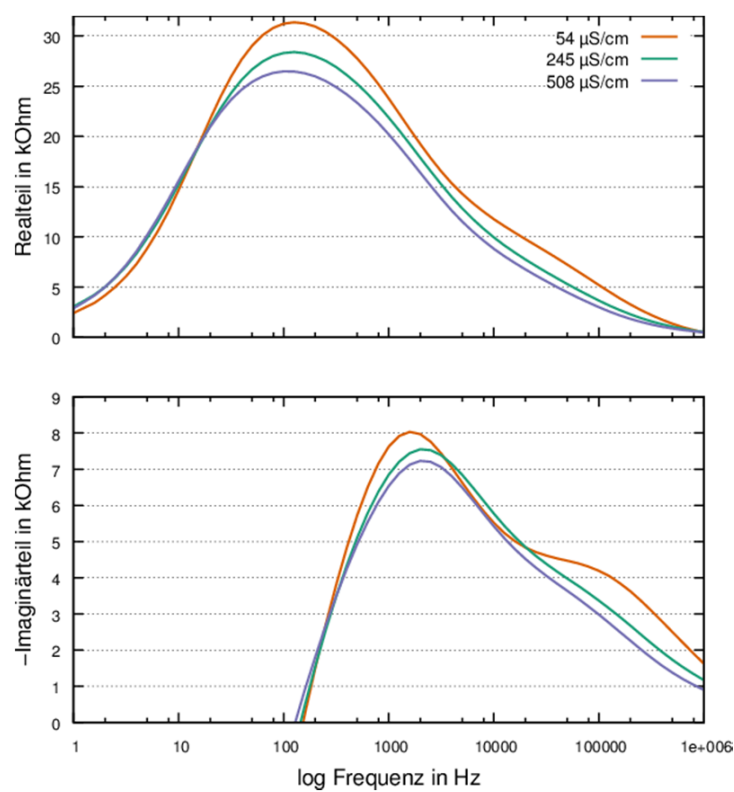

Steckling 9
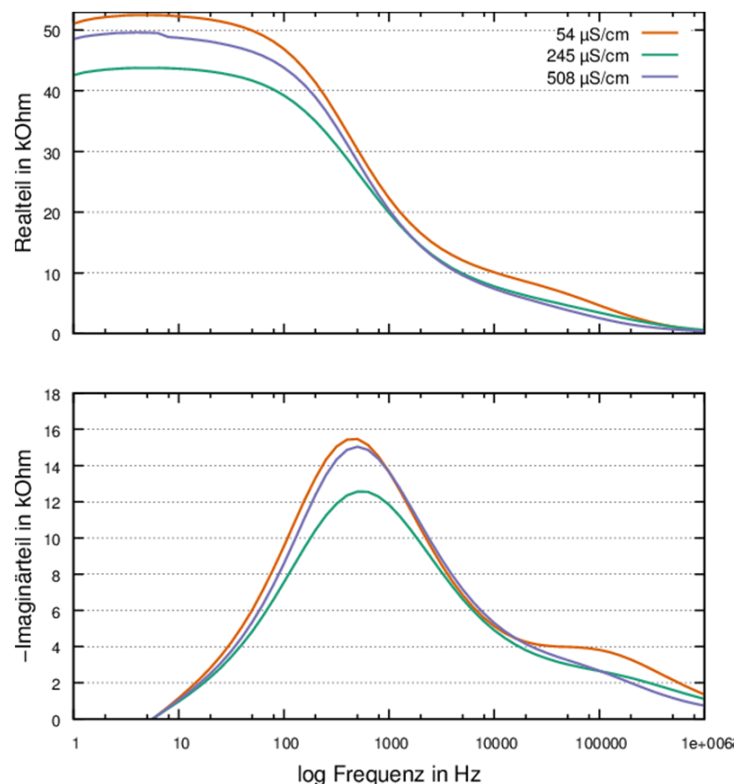

Steckling 8
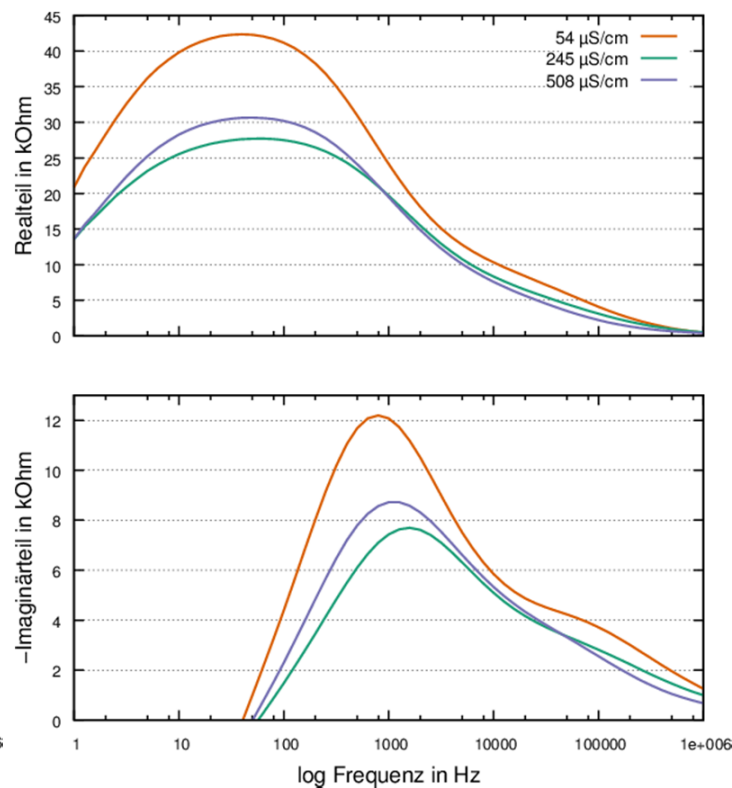

Steckling 10
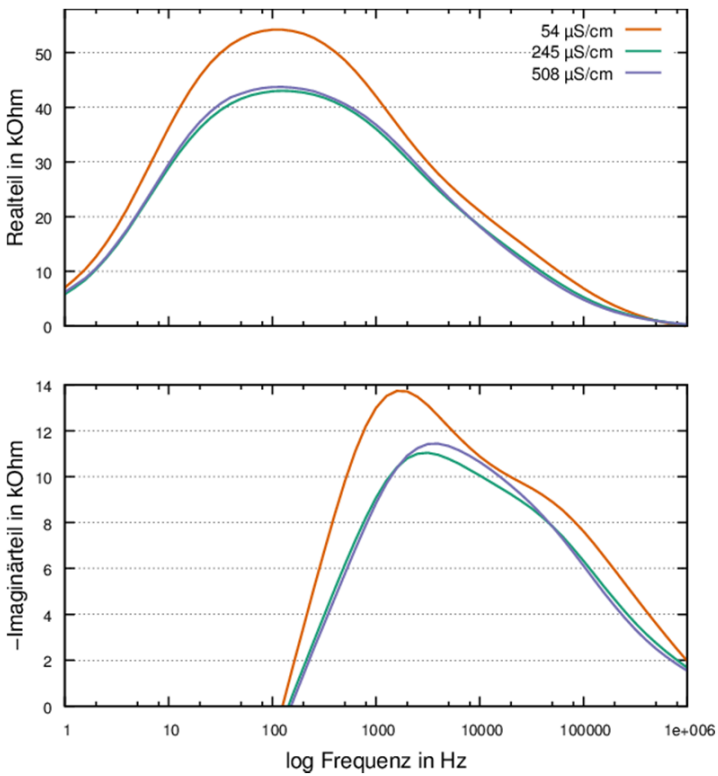

Abbildung A.3: Ergebnisse der Untersuchungen zum Einfluss der Leitfähigkeit der Nährlösung für die Stecklinge 7 bis 10 . 
Steckling 11
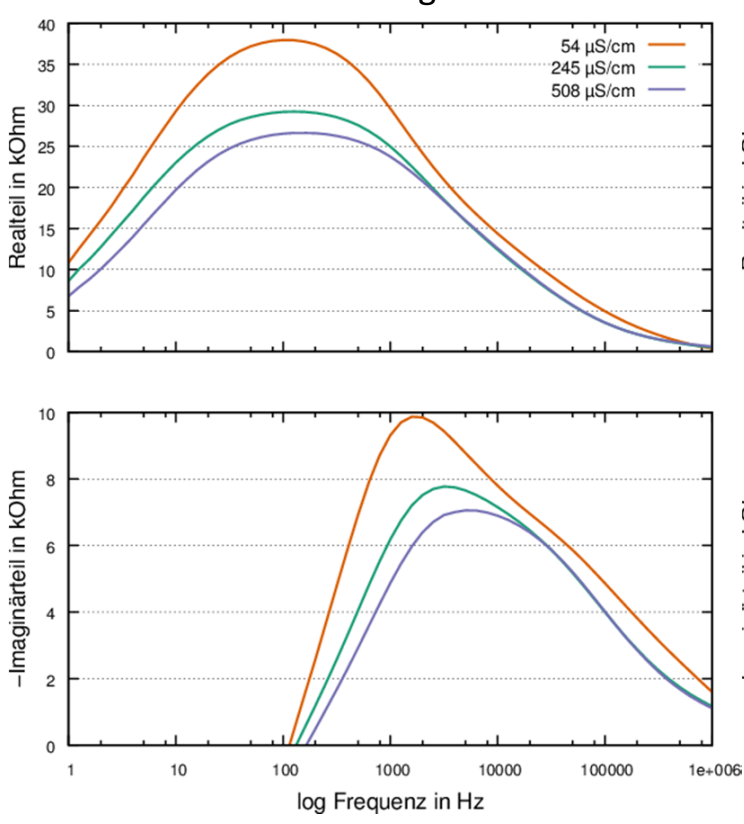

$\log$ Frequenz in $\mathrm{Hz}$

Steckling 13
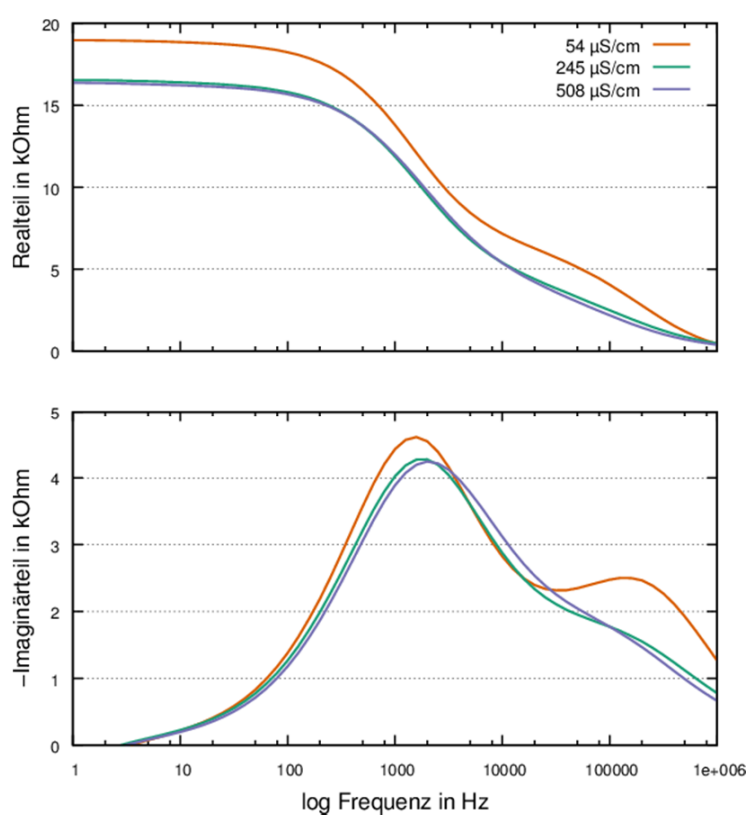

Steckling 12
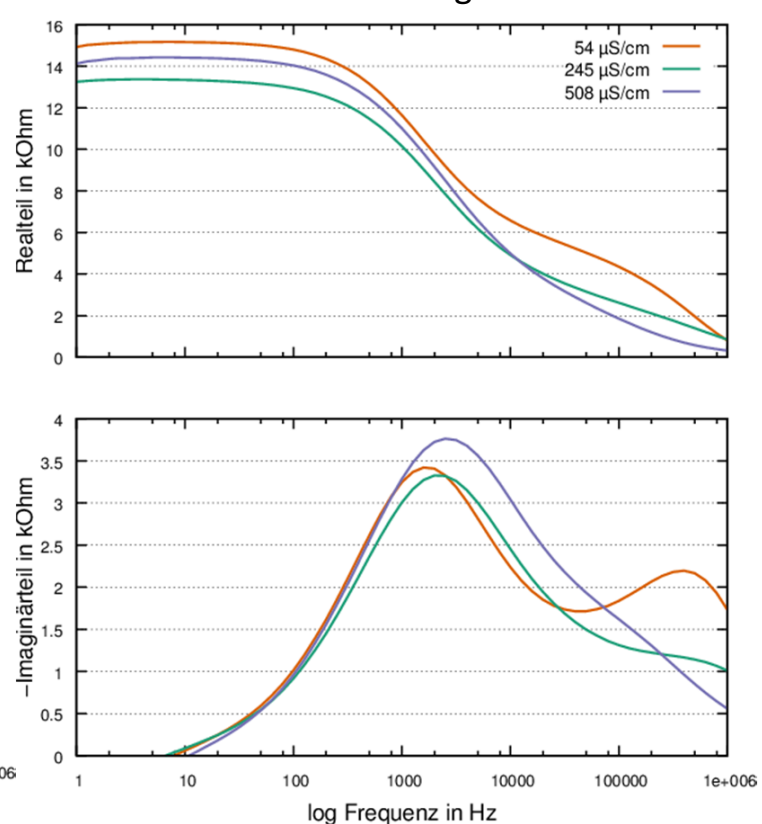

Steckling 14
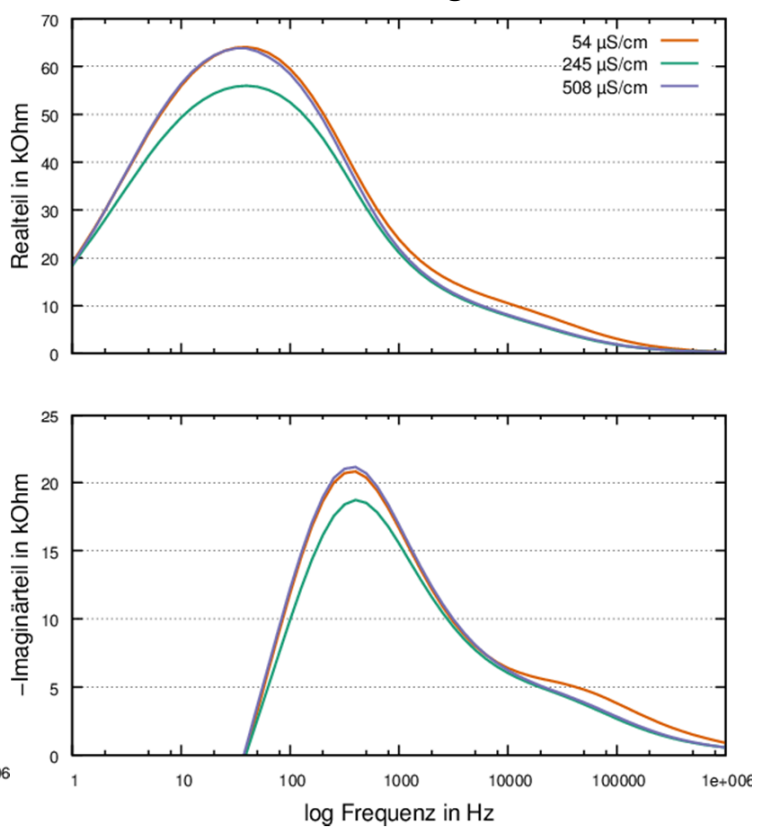

Abbildung A.4: Ergebnisse der Untersuchungen zum Einfluss der Leitfähigkeit der Nährlösung für die Stecklinge 11 bis 14 . 


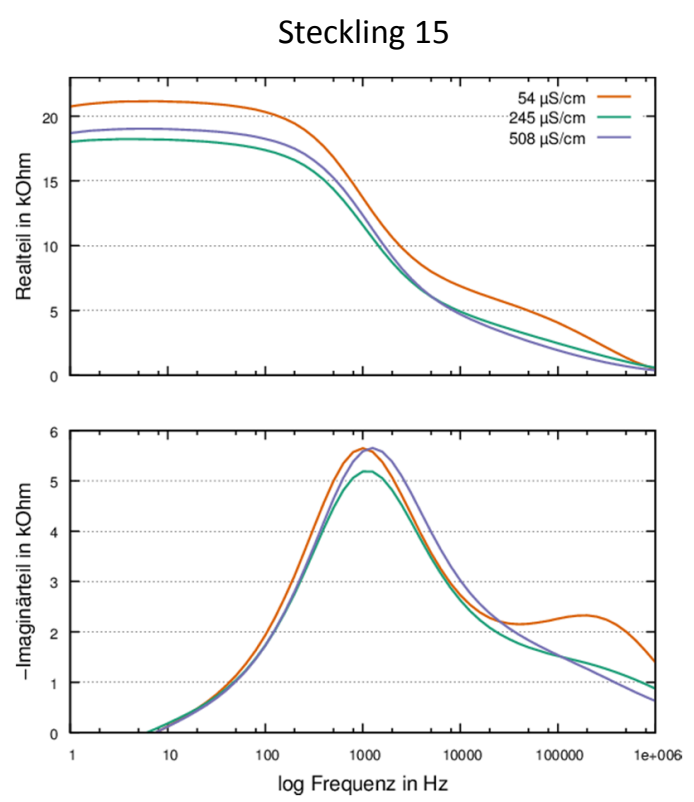

Abbildung A.5: Ergebnisse der Untersuchungen zum Einfluss der Leitfähigkeit der Nährlösung für den Steckling 15. 

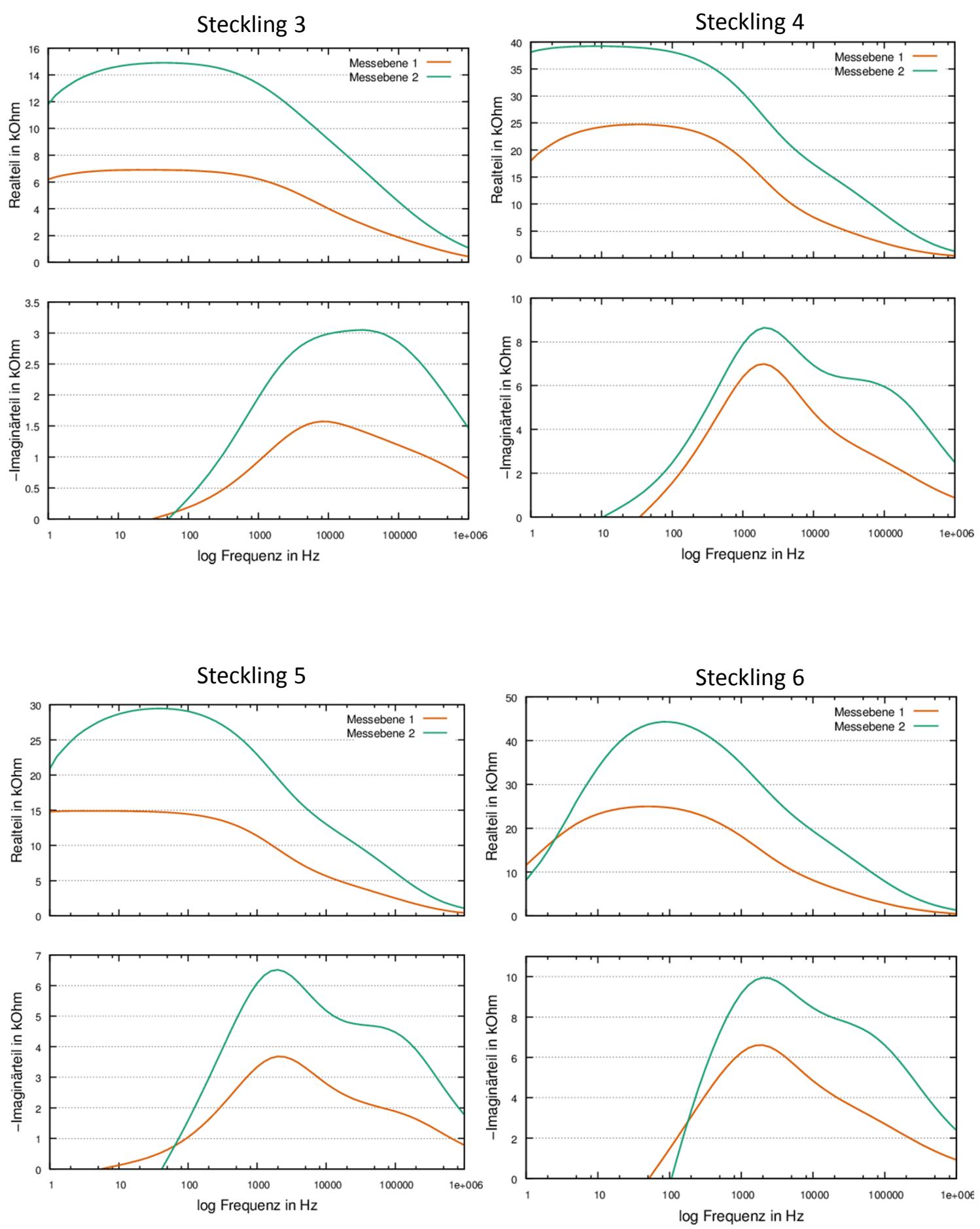

Abbildung A.6: Ergebnisse der Untersuchungen zum Einfluss der Leitgewebe für die Stecklinge 3 bis 6. 
Steckling 7
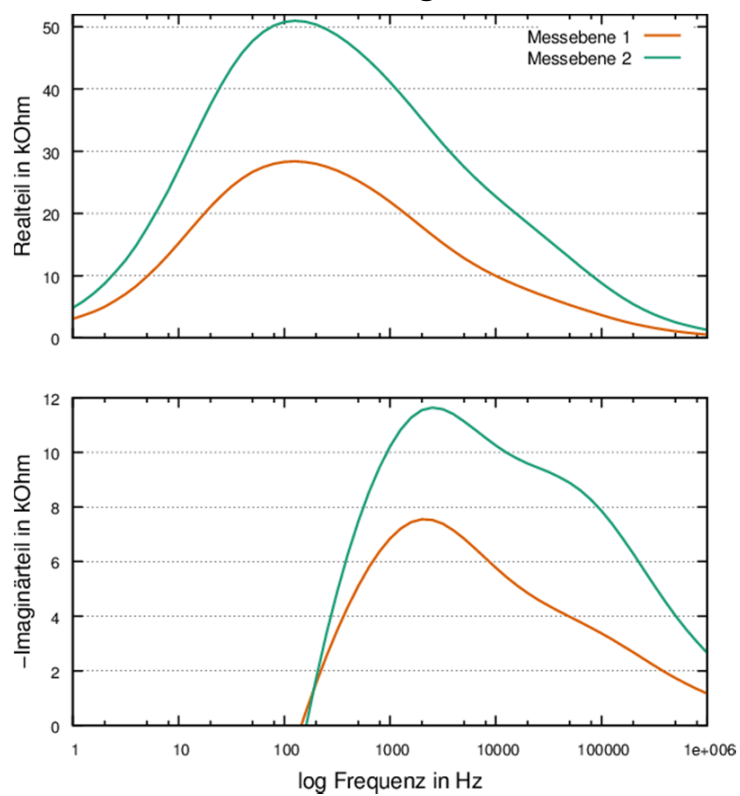

Steckling 9
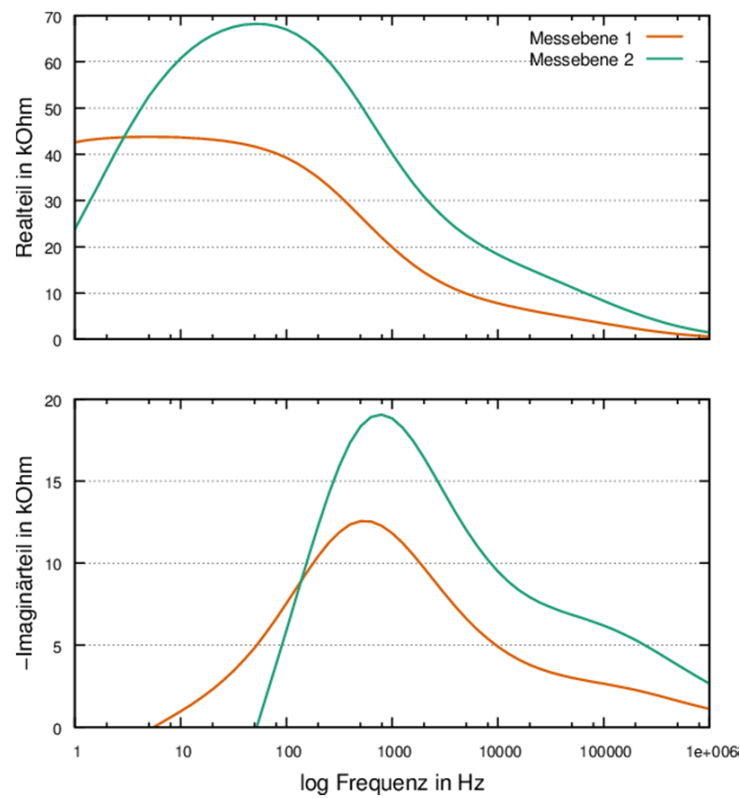

Steckling 8
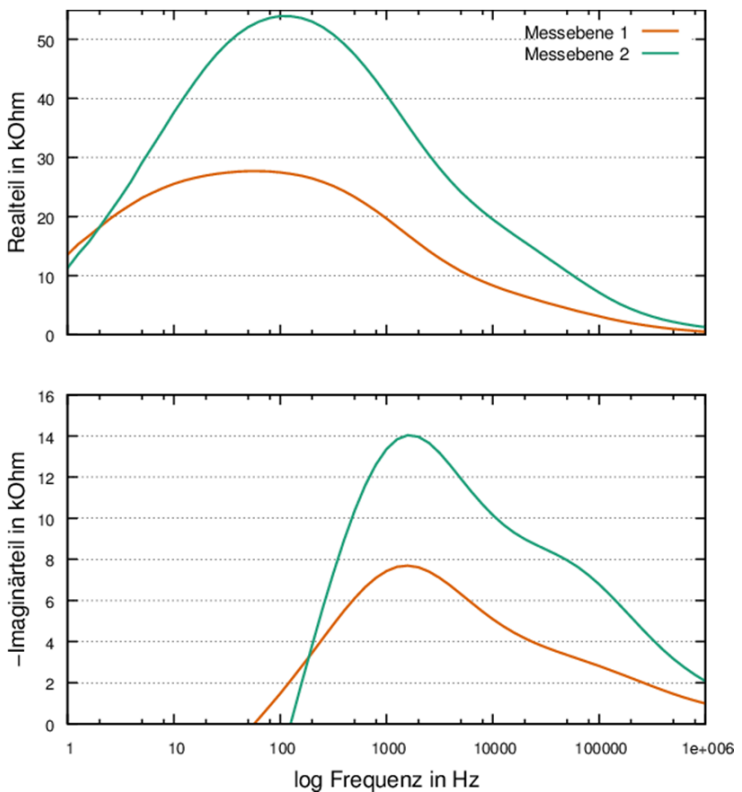

Steckling 10
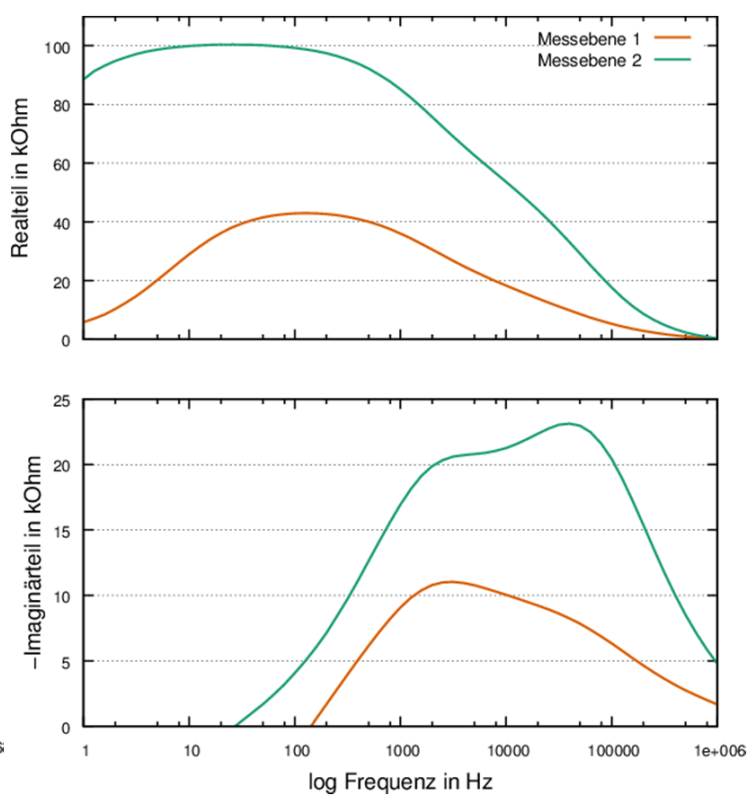

Abbildung A.7: Ergebnisse der Untersuchungen zum Einfluss der Leitgewebe für die Stecklinge 7 bis 10. 
Steckling 11
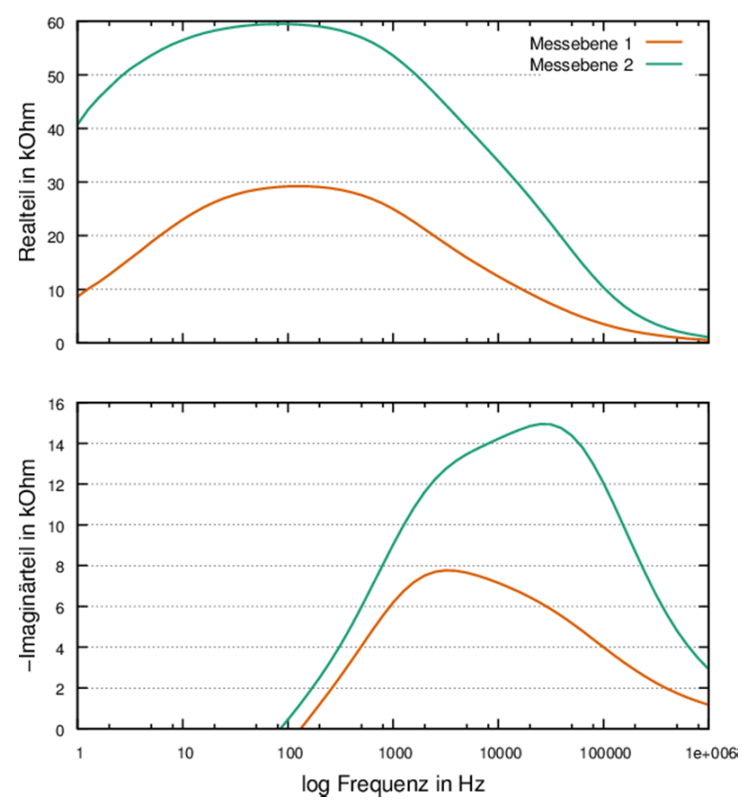

Steckling 13
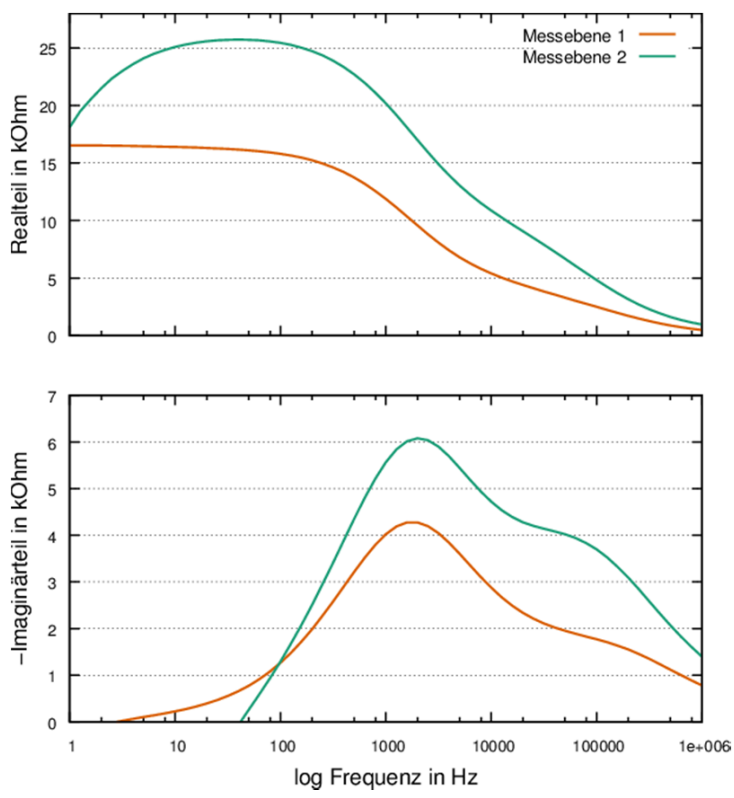

Steckling 12
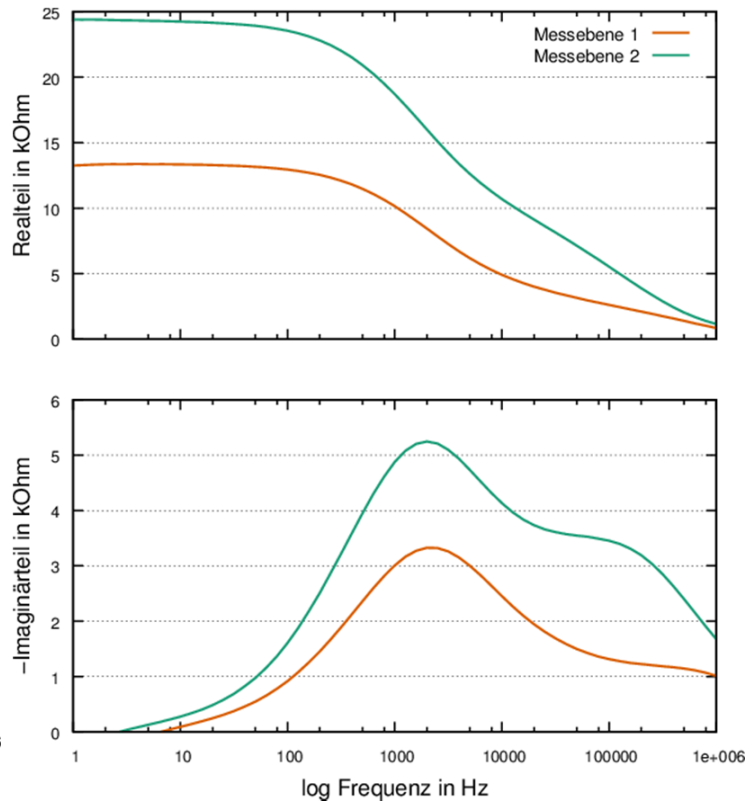

Steckling 14
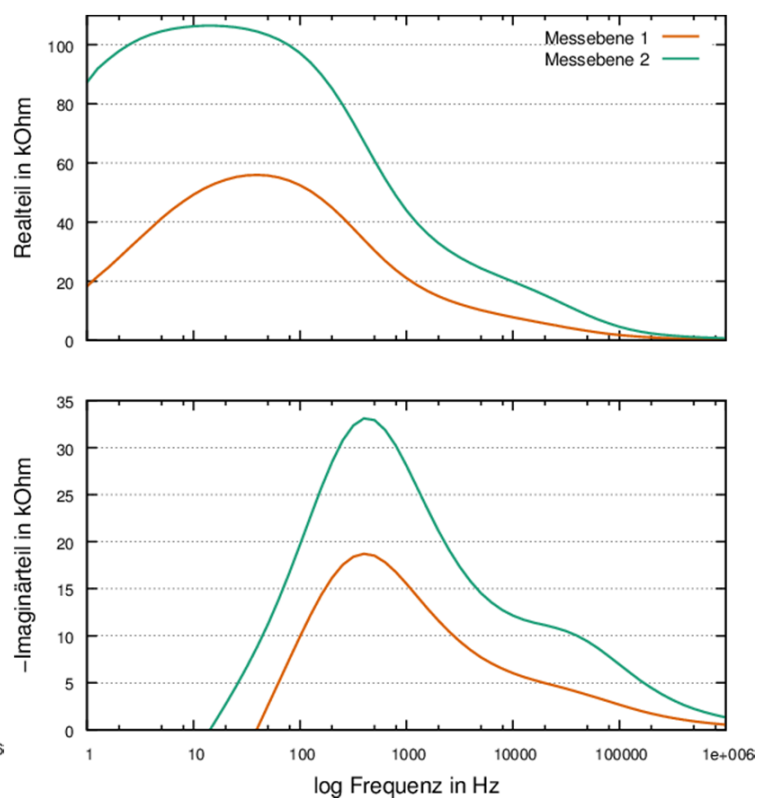

Abbildung A.8: Ergebnisse der Untersuchungen zum Einfluss der Leitgewebe für die Stecklinge 11 bis 14 . 

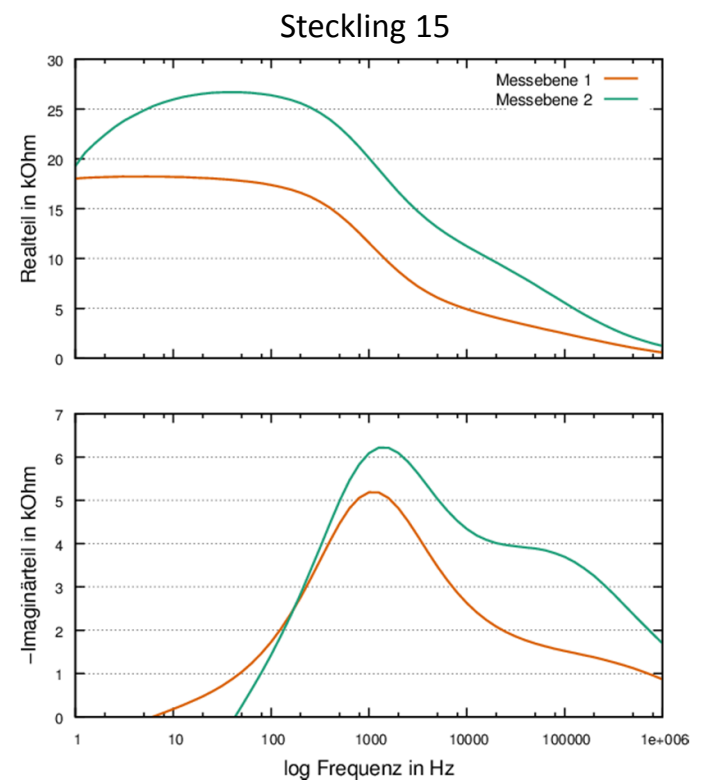

Abbildung A.9: Ergebnisse der Untersuchungen zum Einfluss der Leitgewebe für den Steckling 15 . 
Family: gaussian

Link function: identity

Formula:

Länge $\sim s(R 1, b s=" c s ")+s(C 1, b s=" c s ")+s(R 2, b s=" c s ")+$ $\mathrm{s}(\mathrm{C} 2, \mathrm{bs}=$ "cs") $+\mathrm{s}(\mathrm{R} 3, \mathrm{bs}=$ "cs" $)+\mathrm{s}(\mathrm{C} 3, \mathrm{bs}=$ "cs" $)+$ $s(R 4, b s=" c s ")+s(C 4, b s=" c s ")+s(R \Theta, b s=" c s ")+$ factor(Steckling)

Parametric coefficients:

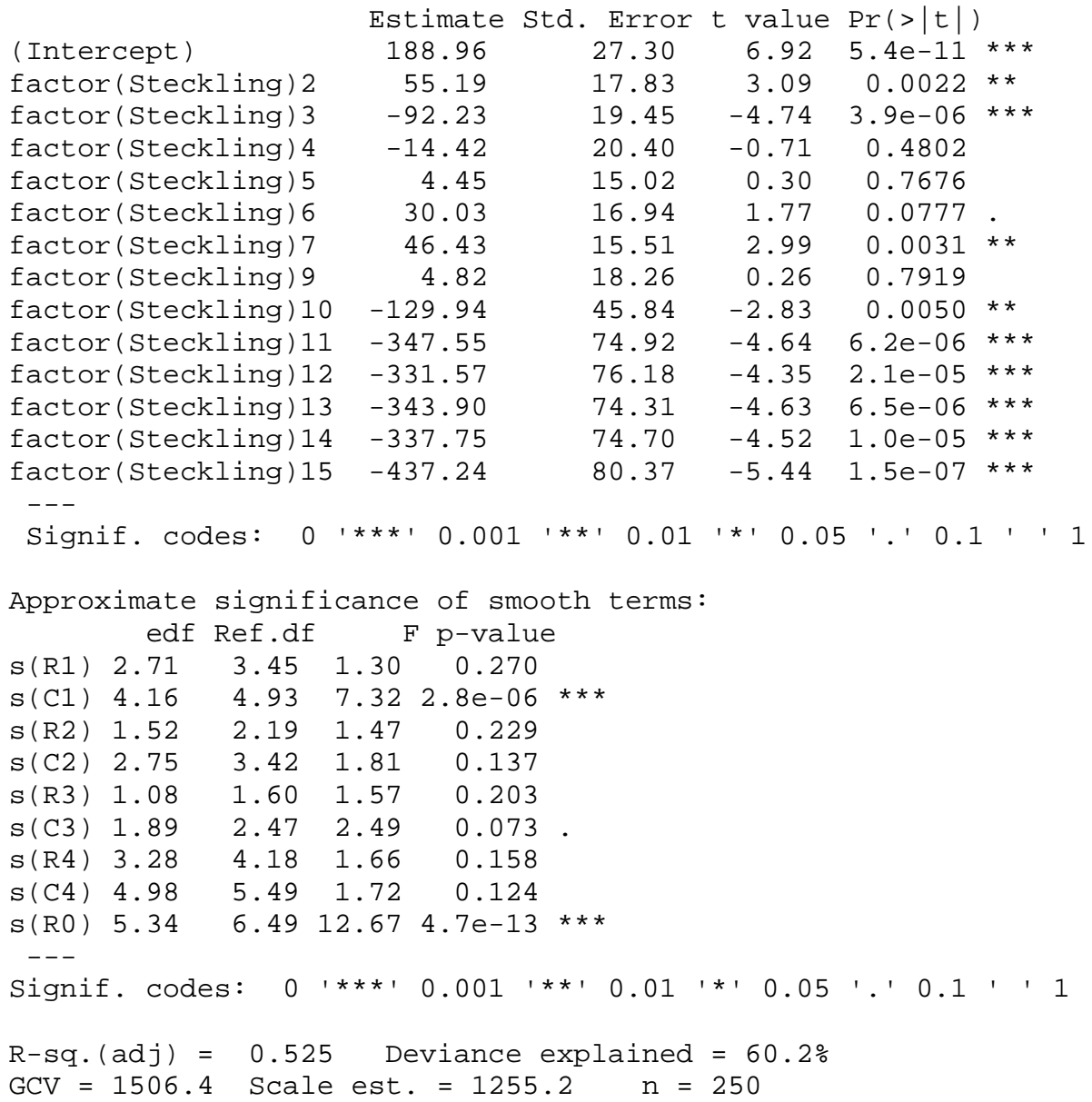

Abbildung A.10: Statistische Auswertung der multivariaten Analyse für die DreipunktModelldaten von Hauptversuch 1. 


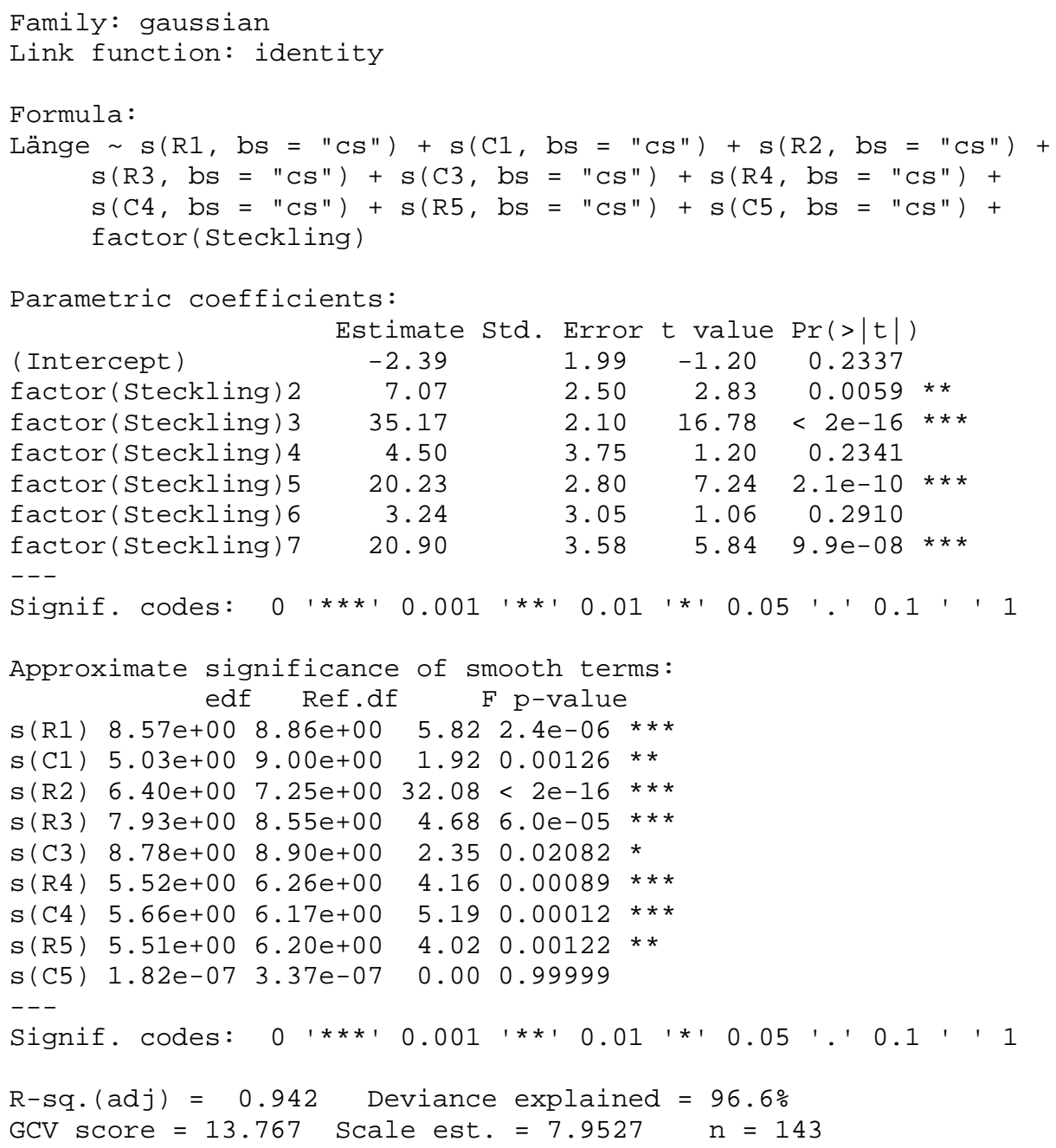

Abbildung A.11: Statistische Auswertung der multivariaten Analyse für die ZweipunktModelldaten von Hauptversuch 2. 
Family: gaussian

Link function: identity

Formula:

Länge $\sim s(R 1, b s=" c s ")+s(C 1, b s=" c s ")+s(R 2, b s=" c s ")+$ $\mathrm{s}(\mathrm{R} 3, \mathrm{bs}=$ "cs" $)+\mathrm{s}(\mathrm{C3}, \mathrm{bs}=$ "cs" $)+\mathrm{s}(\mathrm{R} 4, \mathrm{bs}=$ "cs" $)+$ $\mathrm{s}(\mathrm{C} 4, \mathrm{bs}=$ "cs") + $\mathrm{s}(\mathrm{R} 5, \mathrm{bs}=$ "cs") $+\mathrm{s}(\mathrm{C5}, \mathrm{bs}=" \mathrm{cs} ")+$ factor(Steckling)

Parametric coefficients:

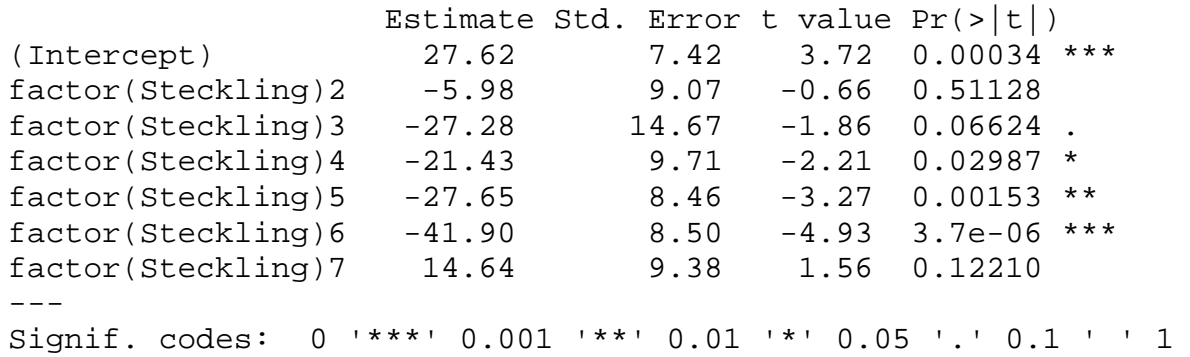

Approximate significance of smooth terms: edf Ref.df $F$ p-value $\mathrm{s}$ (R1) $7.03 \mathrm{e}+0 \odot 7.87 \mathrm{e}+0 \odot \quad 3.360 .00213$ * $\mathrm{s}(\mathrm{C} 1) \quad 6.36 \mathrm{e}-08 \quad 1.18 \mathrm{e}-07 \quad 0.01 \quad 0.99997$ s(R2) $6.10 \mathrm{e}+0 \odot \quad 6.98 \mathrm{e}+0 \odot \quad 8.50 \quad 2.8 \mathrm{e}-08 * * *$ $\mathrm{S}(\mathrm{R} 3) \quad 3.94 \mathrm{e}+00 \quad 4.87 \mathrm{e}+007.74 \quad 4.7 \mathrm{e}-06 * * *$ $\mathrm{s}(\mathrm{C} 3) \quad 3.13 \mathrm{e}+00 \quad 3.72 \mathrm{e}+0 \odot \quad 1.76 \quad 0.14744$ $\mathrm{s}(\mathrm{R} 4) \quad 7.86 \mathrm{e}+0 \odot \quad 8.29 \mathrm{e}+00 \quad 4.45 \quad 0.00011$ *** $\mathrm{s}(\mathrm{C} 4) \quad 7.81 \mathrm{e}+008.28 \mathrm{e}+005.605 .8 \mathrm{e}-06 * * *$ S(R5) $1.93 e+002.21 e+005.620 .00400$ ** $\mathrm{s}(\mathrm{C5}) 8.38 \mathrm{e}+008.82 \mathrm{e}+005.961 .4 \mathrm{e}-06$ *** - - -

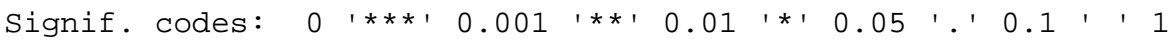
$\mathrm{R}-\mathrm{sq} \cdot(\operatorname{adj})=0.914$ Deviance explained $=94.5 \%$ GCV score $=18.854$ Scale est. $=11.891 \quad \mathrm{n}=144$

Abbildung A.12: Statistische Auswertung der multivariaten Analyse für die DreipunktModelldaten von Hauptversuch 2. 